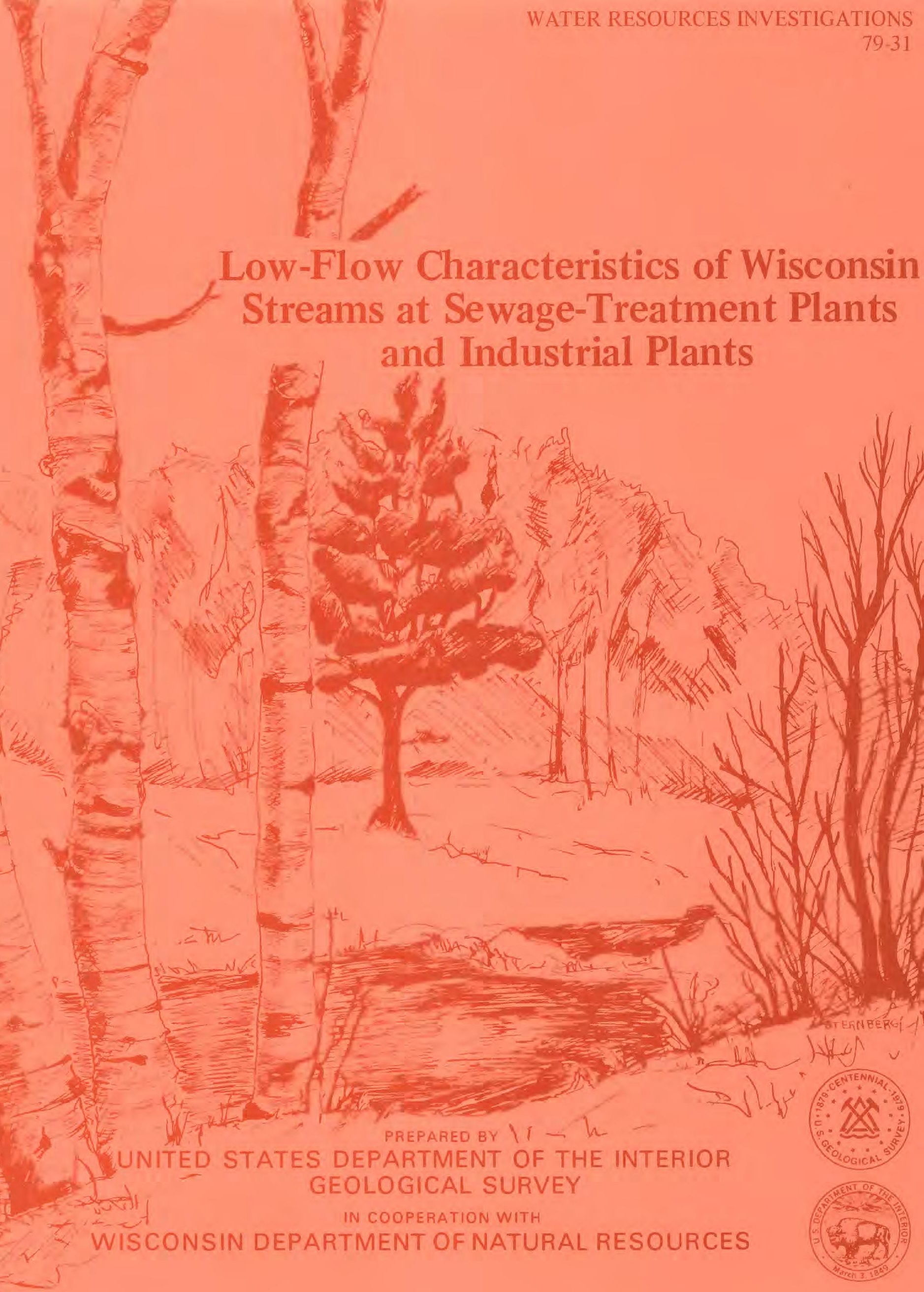




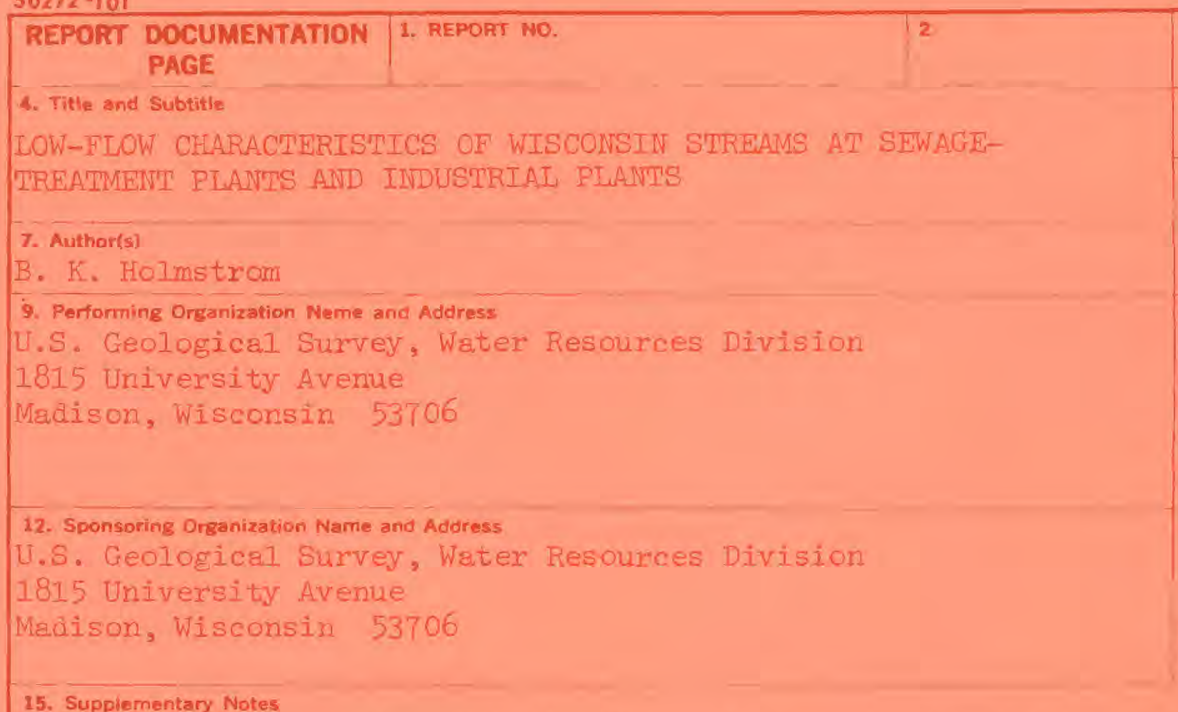

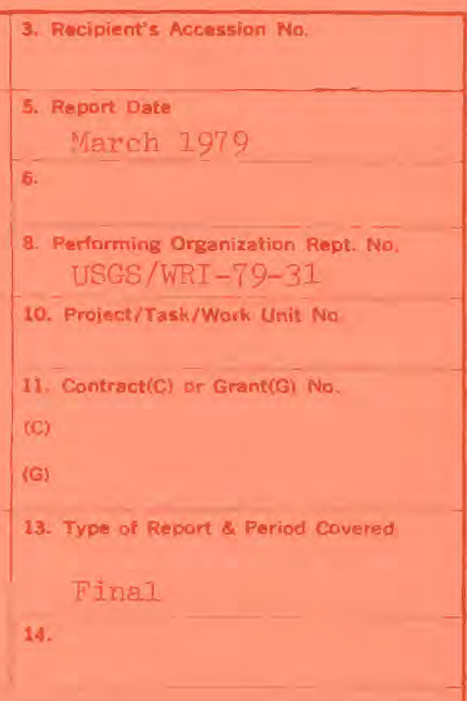

Prepared in cooperation with the Wisconsin Department of Natural Resource

\section{Abstract (Limit: 200 words)}

The purpose of this report is to present low-flow characteristics of lisconsin streams at sewage-treatment and industrial plants. The low-flow characteristics axe needed to implement water-quality standards for all surface waters of the state. Water-quality standards in Wisconsin are based on the annual minimum 7-day mean flow below which the flow will fall on the average of once in 10 years $\left(Q_{7}, 10\right)$.

Low-flow characteristies of Wisconsin streams at 397 sewage-treatment plants anw 143 industriel plants in 30 river basins are presented. The low-flow characteristics presented axe the antual minimum 7-day mean flow below which the flow will fall bo the average of once in 2 years $(27,2)$ and the 87,10 . The 27,2 values ranfed from $D$ to $3,770 \mathrm{ft} 3 / \mathrm{s}$ and $Q 7,10$ values ranged from 0 to $2,790 \mathrm{ft}^{3} / \mathrm{s}$.

The sccuracy of the estimated low-flow characteristics is evaluated accordine to the type and amount of data used to determine the low-flow characteristics. The accuracy determined by the standard error of estimate for the 10-year low flow ranged from an average of 18 percent at continuous-record gaging stations to an averame of 46 percent where only three base-flow measurements were available.

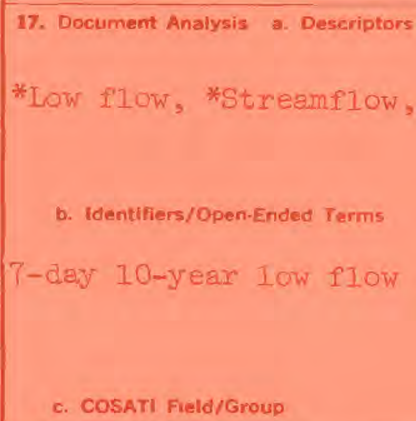

19. Security Class (This Report)




\title{
Low-Flow Characteristics of Wisconsin Streams at Sewage-Treatment Plants and Industrial Plants
}

\author{
B. K. HOLMSTROM
}

U. S. GEOLOGICAL SURVEY

Water Resources Investigations 79-31

Prepared in cooperation with the

Wisconsin Department of Natural Resources

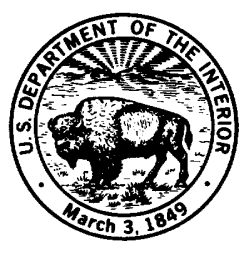

MARCH 1979 


\title{
UNITED STATES DEPARTMENT OF THE INTERIOR
}

\author{
CECIL D. ANDRUS, SECRETARY
}

\section{GEOLOGICAL SURVEY}

H. William Menard, Director

For additional information write to:

U. S. Geological Survey

1815 University Avenue

Madison, Wisconsin 53706 


\section{CONTENTS}

Page

Abstract-_- 1

Introduction-_-_-_-_-_ 2

Low-flow characteristics-_-____-__-__-_ 2

Analytical techniques-______ 4

Gaging stations- 4

Low-flow partial-record stations-_- 4

Project stations-_-_-_-_-_-_ 5

Accuracy-_-_-_-_-_-_ 5

Gaging stations-_-_- 5

Low-flow partial-record stations-_- 6

Project stations- 7

References-_- 8

Index-_- 110

\section{ILLUSTRATIONS}

Page

Figure 1. Map showing location of river basins used in this report-_-_-_-_-_-_- 3

2. Graph showing method of estimating $Q_{7}, 2$ and $Q_{7}, 10$ at
project stations-

\section{TABLES}

Page

Table 1. Bad Axe River basin station locations and low-flow characteristics-_-

2. Baraboo-Lemonweir River basin station locations and lowflow characteristics-_-_-_-

3. Black River basin station locations and low-flow characteristics-_-_-_-

4. Lower Chippewa River basin station locations and low-flow characteristics-_-_-_-_-

5. Upper Chippewa River basin station locations and low-flow characteristics-_-_-_-_-_-_-

6. Des Plaines-Pike River basin station locations and lowflow characteristics-

7. Duck Creek-Pensaukee River basin station locations and low-flow characteristics-_-_-_-

8. Fox River (Illinois) basin station locations and low-flow characteristics

9. Lower Fox River basin station locations and low-flow characteristics-_-_-_-_-_-_- 
10. Upper Fox River basin station locations and low-flow characteristics-_-_._- 40

11. Grant-Platte River basin station locations and low-flow characteristics-_-_-_-

12. La Crosse River basin station locations and low-flow characteristics-_-_._- 48

13. Manitowoc River basin station locations and low-flow characteristics-_._. 50

14. Menominee River basin station locations and low-flow characteristics-_- 53

15. Milwaukee River basin station locations and low-flow characteristics-_-_-_-

16. Oconto River basin station locations and low-flow characteristics- 56

17. Pecatonica River basin station locations and low-flow characteristics-_-_-_-

18. Peshtigo River basin station locations and low-flow

19. Lower Rock River basin station locations and low-flow

20. Upper Rock River basin station locations and low-flow characteristics--_-_-

21. Root River basin station locations and low-flow characteristics-_-_-_-_-

22. St. Croix River basin station locations and low-flow

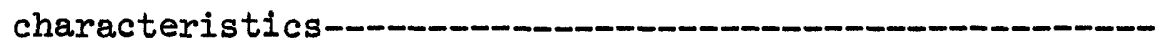

23. Sheboygan River basin station locations and low-flow characteristics-_-_-_-_-_-

24. Sugar River basin station locations and low-flow

25. Lake Superior basin station locations and low-flow characteristics-_-_-

26. Trempealeau-Buffalo River basin station locations and low-flow characteristics--_-_-

27. Twin-Kewaunee River basin and Door County station locations and low-flow characteristics-_-

28. Lower Wisconsin River basin station locations and low-flow characteristics-_-

29. Upper Wisconsin River basin station locations and low-flow characteristics-_-

30. Wolf River basin station locations and low-flow characteristics-_-_-_-

31. Accuracy of estimated low-flow characteristics for several methods-_._.

32. Standard error of estimate for the 10-year low-flow event $\left(\mathrm{SE}_{7,10}\right)$ for Wisconsin basins at low-flow partial-record stations 


\title{
Low-Flow Characteristics of Wisconsin Streams at Sewage-Treatment Plants and Industrial Plants
}

\author{
B. K. HOLMSTROM
}

\begin{abstract}
Low-flow characteristics of Wisconsin streams at 397 sewage-treatment plants and 143 industrial plants in 30 river basins are presented in this report. The low-flow characteristics are the annual minimum 7-day mean flow below which the flow will fall on the average of once in 2 years $(Q 7,2)$ and the annual minimum 7 -day mean flow below which the flow will fali on the average of once in 10 years $\left(Q_{7,10}\right)$.

The low-flow characteristics at most sewage-treatment and industrial plants (project stations) were determined by correlating base-flow measurements at the project stations with the concurrent daily mean flow at continuous-record gaging stations in the area. The Q7,2 and $Q_{7,10}$ discharges determined by a frequency analysis at the continuous-record gaging stations were used to estimate the Q7,2 and 47,10 at the project stations. For some project stations the low-flow characteristics also were determined by frequency analysis at nearby continuous-record gaging stations or correlation analysis at nearby low-flow partial-record stations. The 47,2 values ranged from 0 to $3,770 \mathrm{ft} 3 / \mathrm{s}$ and $Q_{7,10}$ values ranged from 0 to $2,790 \mathrm{ft} 3 / \mathrm{s}$.

The accuracy of the estimated low-flow characteristics is evaluated according to the type and amount of data used to determine the low-flow characteristics. The accuracy determined by the standard error of estimate for the 10-year low flow $\left(\mathrm{SE}_{7}, 10\right)$ ranged from an average of 18 percent at continuous-record gaging stations to an average of 46 percent when only three base-flow measurements were available.
\end{abstract}




\section{INTRODUCTION}

Low-flow characteristics of Wisconsin streams at sewage-treatment and industrial plants are needed by the Wisconsin Department of Natural Resources to implement water-quality standards for all surface waters of the State. The implementation requires that the sewage effluent from all waste sources has to be evaluated in relation to the low-flow characteristics of the receiving stream. Water-quality standards in Wisconsin are based on the annual minimum 7-day mean flow below which the flow will fall on the average of once in 10 years $\left(Q_{7,10}\right)$.

The purpose of this report is to describe the low-flow characteristics of Wisconsin streams at sewage-treatment and industrial plants. This report contains estimates of the 47,10 along with estimates of the annual minimum 7-day mean flow below which the flow will fall on the average of once in 2 years $\left(Q_{7,2}\right)$ at sites in 30 basins in Wisconsin (fig. 1).

The report includes: estimates of the magnitude and frequency of recurrence of low flow for sites where streamflow data have been collected, low-flow discharge measurements at these sites, an explanation of methods used to determine the low-flow characteristics, and average standard error of estimates based on methods used to determine the low-flow characteristics.

This study was done in cooperation with the Wisconsin Department of Natural Resources. This is the final report in the series and low-flow estimates in tables 1-30 supersede earlier estimates by Gebert and Holmstrom (1974) and Holmstrom (1977).

For the convenience of readers who may want to use the International System of Units, the data presented in the tables may be converted from inch/pound units by using the following factors:

Multiply

mile (mi)

foot (ft)

square mile $\left(\mathrm{mi}^{2}\right)$

cubic foot per second $\left(\mathrm{ft}^{3} / \mathrm{s}\right)$
By

1.609

.3048

2.59

.02832
To obtain

kilometer $(\mathrm{km})$ meter (m) square kilometer $\left(\mathrm{km}^{2}\right)$ cubic meter per second $\left(\mathrm{m}^{3} / \mathrm{s}\right)$

\section{LOW-FLOW CHARACTERISTICS}

The estimated low-flow characteristics at 397 sewage-treatment plants and 143 industrial plants are listed in tables 1-30. Information included in the tables for each station are project station number; station location; drainage area; measured discharge; and accuracy level of the estimated lowflow characteristics. The station number is based on the number assigned by the Wisconsin Department of Natural Resources (DNR) in reports on pollutioninvestigation surveys for each basin. The number used in the DNR reports 


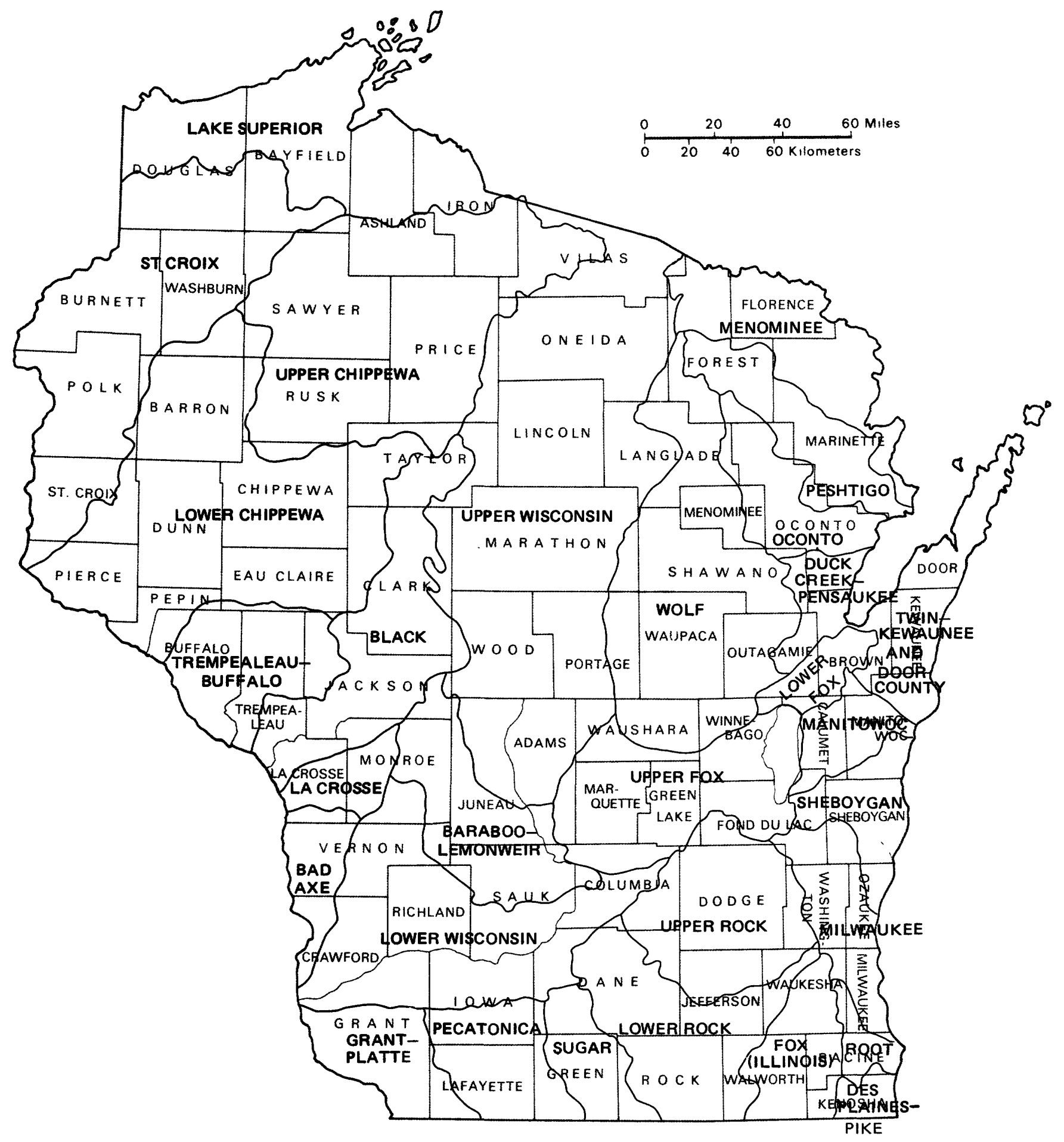

Figure 1. Location of river basins used in this report. 
has been modified by the addition of a letter before each number to help identify the basin. The Department of Natural Resources could change the station number when the reports are updated; therefore, the year of the report using these numbers is shown in each table.

Alternate measuring sites were selected at stations where the stream was dry or ponded. Measurements were obtained at a downstream location if it appeared that substantial natural inflow had entered the stream. When the sewage-treatment or industrial plant discharged effluent into a ponded stream or into a dry streambed, measurements for both the sewage-treatment or industrial plant station and the alternate site were included in the table.

\section{ANALYTICAL TECHNIQUES}

The low-flow characteristics in tables 1-30 were determined by three methods of analyses. These methods depended on the type of data available at or near the project station. The 3 basic types of data available are: (1) continuous record of daily streamflow (continuous-record gaging stations); (2) 7 to 36 base-flow discharge measurements (low-flow partial-record stations); (3) 3 to 11 base-flow discharge measurements (project stations). A description of the analysis for the types of stations follows.

\section{GAGING STATIONS}

Where a sewage-treatment or industrial plant was located close to a continuous-record gaging station, the low-flow characteristics determined at the gaging station were used for the site at the sewage-treatment or industrial plant.

The low-flow characteristics at gaging stations were determined by a frequency analysis of the recorded 7-day annual minimum flows using a logPearson Type III probability distribution or a plotting position analysis (Riggs, 1972). If the results of the two analyses were substantially different, the plotting position analysis was used to determine the 47,2 and 07,10 discharge. All the available data for the gaging station through the 1975 water year were used for this analysis.

\section{LOW-FLOW PARTIAL-RECORD STATIONS}

Where a sewage-treatment or industrial plant was located near a lowflow partial-record station, the low-flow characteristics determined for the partial-record station were used at the station. The low-flow characteristics at the station were determined from a relation line established by correlating 7 to 36 base-flow discharge measurements at these stations with the concurrent discharge at continuous-record gaging stations in the area (Gebert, 1971). The $Q_{7,2}$ and $Q_{7,10}$ at the continuous-record gaging stations were then transferred through the relation line to estimate $Q_{7,2}$ and $Q_{7,10}$ for
the partial-record station. 


\section{PROJECT STATIONS}

Most of the estimated low-flow characteristics listed in this report are based on 3 to 11 base-flow measurements at each project station. The base-flow measurements at these sites were correlated with concurrent daily mean flow at continuous-record gaging stations in the area. The slope of the relation line was compared to the established relation line at nearby low-flow partial-record stations and other project stations for uniformity. If the factors that largely influence low flow are uniform in the area, the relation lines should have the same slope. If the relation line at the project station was defined by discharge measurements having significant scatter, the relation line was adjusted to agree more closely with the better established relation line at the low-flow partial-record station.

The $Q_{7,2}$ and $Q_{7,10}$ discharges, determined by a frequency analysis at the continuous-record gaging stations, were then transferred through the relation line to estimate the $Q_{7,2}$ and $Q_{7,10}$ at the project station.

Figure 2 illustrates this method of correlation. The frequency analyses at the gaging stations included the entire period of record at each station through the 1975 water year.

\section{ACCURACY}

The $Q_{7,2}$ and $Q_{7,10}$ values in tables 1-30 are estimates of flow expected in the future. The estimates are based on data collected at each site and analyzed by several methods. Each estimate has an error associated with it, dependent on the amount and kind of data and on the analytical method. The two major sources of error in the estimates are the time-sampling error in collecting the data and the error in the analytical method.

The accuracy associated with different methods used to develop the low-flow estimates is shown in table 31. The accuracy is determined by the standard error of estimate for the 10-year low-flow event ( $\mathrm{SE}_{7}, 10$ ). The estimated low-flow characteristics are within the $\mathrm{SE}_{7,10} 67$ percent of the time and twice the $\mathrm{SE}_{7,10} 95$ percent of the time. An explanation of how the accuracy was determined for each method follows.

\section{GAGING STATIONS}

The accuracy for low-flow characteristics determined from recorded discharge at continuous-record gaging stations was determined according to Hardison (1969). By analyzing all the gaging-station records in Wisconsin, with the assumption that 10 years of record were available at each site, an $\mathrm{SE}_{7,10}$ of 18 percent was determined. This indicates the relative accuracy level associated with sewage-treatment plants that used gaging-station data to define the low-flow characteristics. An accuracy level indicated by the letter "a" appears in tables 1-30 following the low-flow characteristics that have this degree of accuracy. 


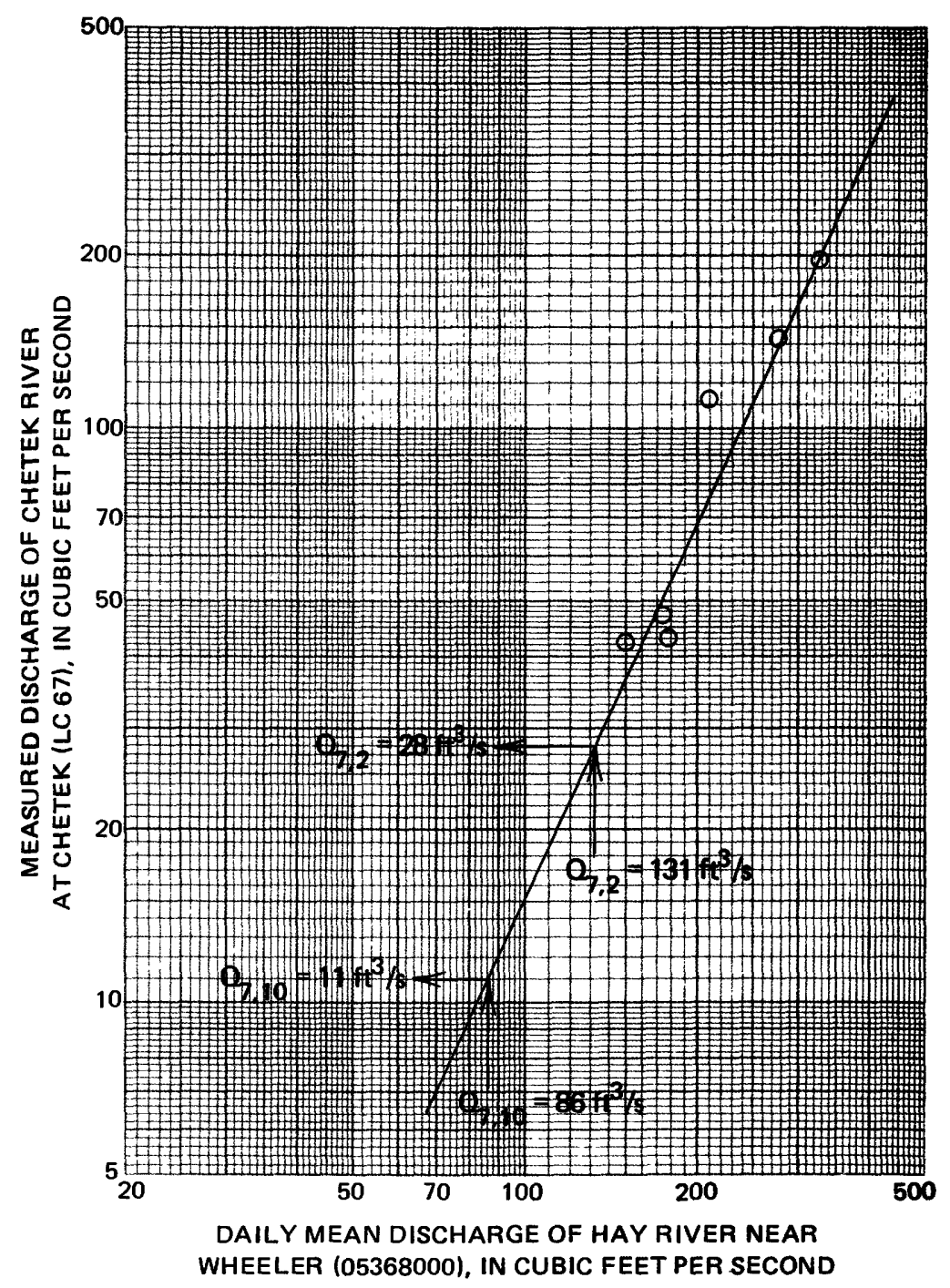

Figure 2. Method of estimating $Q_{7,2}$ and $Q_{7,10}$ at project stations.

\section{LOW-FLOW PARTIAL-RECORD STATIONS}

The accuracy of low-flow characteristics at low-flow partial-record stations can be appraised by a method outlined by Hardison and Moss (1972). Using this procedure an average $\mathrm{SE}_{7,10}$ of 29 percent was determined for 282 low-flow partial-record stations. This $\mathrm{SE}_{7,10}$ is assumed to be typical of the accuracy for project stations where the low-flow characteristics were determined by use of low-flow partial-record station data. Low-flow characteristics in tables 1-30 determined by this method have an accuracy level indicated by the letter "b". 


\section{PROJECT STATIONS}

The accuracy of the low-flow characteristics at project stations cannot be appraised directly. Therefore, the data collected at low-flow partial-record stations were analyzed to approximate the accuracy associated with the low-flow characteristics based on only three base-flow measurements.

Gebert and Holmstrom (1974) found that an $\mathrm{SE}_{7,10}$ of 45 percent should be obtained at the project stations based on correlations using three discharge measurements. However, due to poor measuring conditions during the data-collection period, an $\mathrm{SE}_{7,10}$ of 70 percent was obtained for the Q7,10 estimates for project stations given in the 1974 report (Gebert and Holmstrom, 1974).

The $\mathrm{SE}_{7}, 10$ for the project stations was improved with additional discharge measurements made during good base-flow conditions. Using an analysis similar to that used by Gebert and Holmstrom (1974), 99 low-flow partial-record stations were measured during the same period that streams at project stations were being measured. Estimates of Q7,10 were determined from these discharge measurements using the same procedure used for the project stations. The estimated 47,10 values were plotted against the Q7,10 values based on 9 to 34 discharge measurements. From this relationship the standard error between the two estimates was determined. The overall $\mathrm{SE}_{7,10}$ was then approximated by taking the square root of the sum of the squares of the $\mathrm{SE}_{7,10}$ of the plotted relationship and the $\mathrm{SE}_{7,10}$ determined for the 99 low-flow partial-record stations used in the analysis. This resulted in an $S_{7,10}$ of 46 percent which is approximately the same as the 45 percent determined by Gebert and Holmstrom (1974). Low-flow characteristics in tables $1-30$ determined by this method have an accuracy level indicated by the letter "c".

The accuracy of the low-flow characteristics also differs for different areas of the State. To illustrate this difference in accuracy, values of $\mathrm{SE}_{7,10}$ are listed for Wisconsin basins in table 32. These values of $\mathrm{SE}_{7,10}$ were determined at low-flow partial-record stations and were based on 7 to 36 base-flow measurements during the 1961-77 period. These values at low-flow partial-record stations are probably lower than the $\mathrm{SE}_{7,10}$ associated with low-flow characteristics at the project stations. However, they can be used as a guide to evaluate the relative accuracy of 07,10 estimates at project stations for various basins. For example, the SE7, 10 at low-flow partial-record stations in the Trempealeau-Buffalo River basin is 10 percent. This is considerably lower than the average $\mathrm{SE}_{7,10}$ of 29 percent for all river basins in the state. Therefore, the $\mathrm{SE}_{7}, 10$ for project stations in the Trempealeau-Buffalo River basin probably is considerably less than the $\mathrm{SE}_{7,10}$ of 46 percent determined as the average for the project stations. 


\section{REFERENCES}

Gebert, W. A., 1971, Low-flow frequency of Wisconsin streams: U.S. Geological Survey Hydrologic Investigations Atlas HA-390.

Gebert, W. A., and Holmstrom, B. K., 1974, Low-flow characteristics of Wisconsin streams at sewage-treatment plants: U.S. Geological Survey Water-Resources Investigations 45-74, $101 \mathrm{p}$.

Hardison, C. H., 1969, Accuracy of streamflow characteristics, in Geological Survey Research 1969: U.S. Geological Survey Professional Paper 650-D, p. D210-214.

Hardison, C. H., and Moss, M. E., 1972, Accuracy of low-flow characteristics estimated by correlation of base-flow measurements: U.S. Geological Survey Water-Supply Paper 1542-B, 55 p.

Holmstrom, B. K., 1977, Preliminary estimates of low-flow characteristics of Wisconsin streams at sewage-treatment plants and industrial plants: U.S. Geological Survey administrative report, $74 \mathrm{p}$.

Riggs, H. C., 1972, Low-flow investigations: Techniques of Water-Resources Investigations of the U.S. Geological Survey, Book 4, Chapter BI, $18 \mathrm{p}$.

Wisconsin Department of Natural Resources, 1966, Report on an investigation of the pollution in the Lake Superior drainage basin made during 1965 and early 1966: Madison, Wis., 15 p., 1 fig., 3 tables.

1967a, Report on an investigation of the pollution in the Des Plaines and Pike basin made during 1966 and 1967: Madison, Wis., 12 p., 1 fig., 3 tables.

1967b, Report on an investigation of the pollution in the upper Fox River basin made during 1966 and early 1967: Madison, Wis., 21 p., 1 fig., 3 tables.

1967c, Report on an investigation of the pollution in the Root River basin made during 1966 and 1967: Madison, Wis., 17 p., 1 fig., 3 tables.

1968a, Report on an investigation of the pollution in the lower Fox River and Green Bay made during 1966 and 1967: Madison, Wis., 47 p., 11 figs., 13 tables.

1968b, Report on an investigation of the pollution in the Milwaukee River basin made during 1966 and 1967: Madison, Wis., 25 p., I fig., 3 tables.

1969a, Report on an investigation of the pollution in the Black River drainage basin made during 1968: Madison, Wis., 17 p., I fig., 4 tables. 
1969b, Report on an investigation of the pollution in the Manitowoc River drainage basin made during 1968: Madison, Wis., 16 p., 1 fig., 4 tables.

1969c, Report on an investigation of the pollution of the Menominee River and its tributaries in Wisconsin made during 1968: Madison, Wis., 13 p., 1 fig., 7 tables.

1969d, Report on an investigation of the pollution in the Oconto River basin made during 1968: Madison, Wis., 10 p., 1 fig., 6 tables.

1969e, Report on an investigation of the pollution of the Peshtigo River and its tributaries made during 1968: Madison, Wis., 11 p., I fig., 6 tables.

1969f, Report on an investigation of the pollution in the upper Rock River drainage basin made during 1967-68: Madison, Wis., 21 p., 1 fig., 5 tables.

1970a, Upper Chippewa River: Madison, Wis., Pollution Investigations Survey, 17 p., 3 figs., 8 tables.

1970b, Sheboygan River: Madison, Wis., Pollution Investigations Survey, 25 p., 3 figs., 4 tables.

1970c, Upper Wisconsin River: Madison, Wis., Pollution Investigations Survey, 42 p., 5 figs., 9 tables.

1971a, Bad Axe basin: Madison, Wis., Pollution Investigations Survey, 16 p., 1 fig., 4 tables.

1971b, Lower Chippewa River: Madison, Wis., Pollution Investigations Survey, 40 p., 3 figs., 10 tables.

1971c, Duck Creek-Pensaukee River: Madison, Wis., Pollution Investigations Survey, 15 p., 1 fig., 3 tables.

1971d, Grant-Platte basin: Madison, Wis., Pollution Investigations Survey, 22 p., 1 fig., 4 tables.

1971e, La Crosse River: Madison, Wis., Pollution Investigations Survey, 17 p., 1 fig., 4 tables.

1971f, Pecatonica River: Madison, Wis., Pollution Investigations Survey, 30 p., 1 fig., 5 tables.

1971g, Lower Rock River: Madison, Wis., Pollution Investigations Survey, 35 p., 4 figs., 5 tables.

1971h, Sugar River: Madison, Wis., Pollution Investigations Survey, 19 p., 1 fig., 6 tables. 
1971i, Twin, Door, and Kewaunee drainage basins: Madison, Wis., Pollution Investigations Survey, 21 p., 4 figs., 4 tables.

1971j, Wolf River: Madison, Wis., Pollution Investigations Survey, 35 p., 3 figs., 7 tables.

1972a, Fox (Illinois) River: Madison, Wis., Pollution Investigations Survey, 29 p., 3 figs., 4 tables.

1972b, St. Croix River: Madison, Wis., Pollution Investigations Survey, 37 p., 2 figs., 5 tables.

1972c, Trempealeau and Buffalo Rivers: Madison, Wis., Pollution Investigations Survey, 35 p., 3 figs., 7 tables.

1972d, Lower Wisconsin River: Madison, Wis., Pollution Investigations Survey, 40 p., 5 figs., 5 tables.

1973, Baraboo and Lemonweir Rivers: Madison, Wis., Pollution Investigations Survey, 29 p., 3 figs., 6 tables. 
Table 1. Bad Axe River basin station locations and low-flow characteristics.

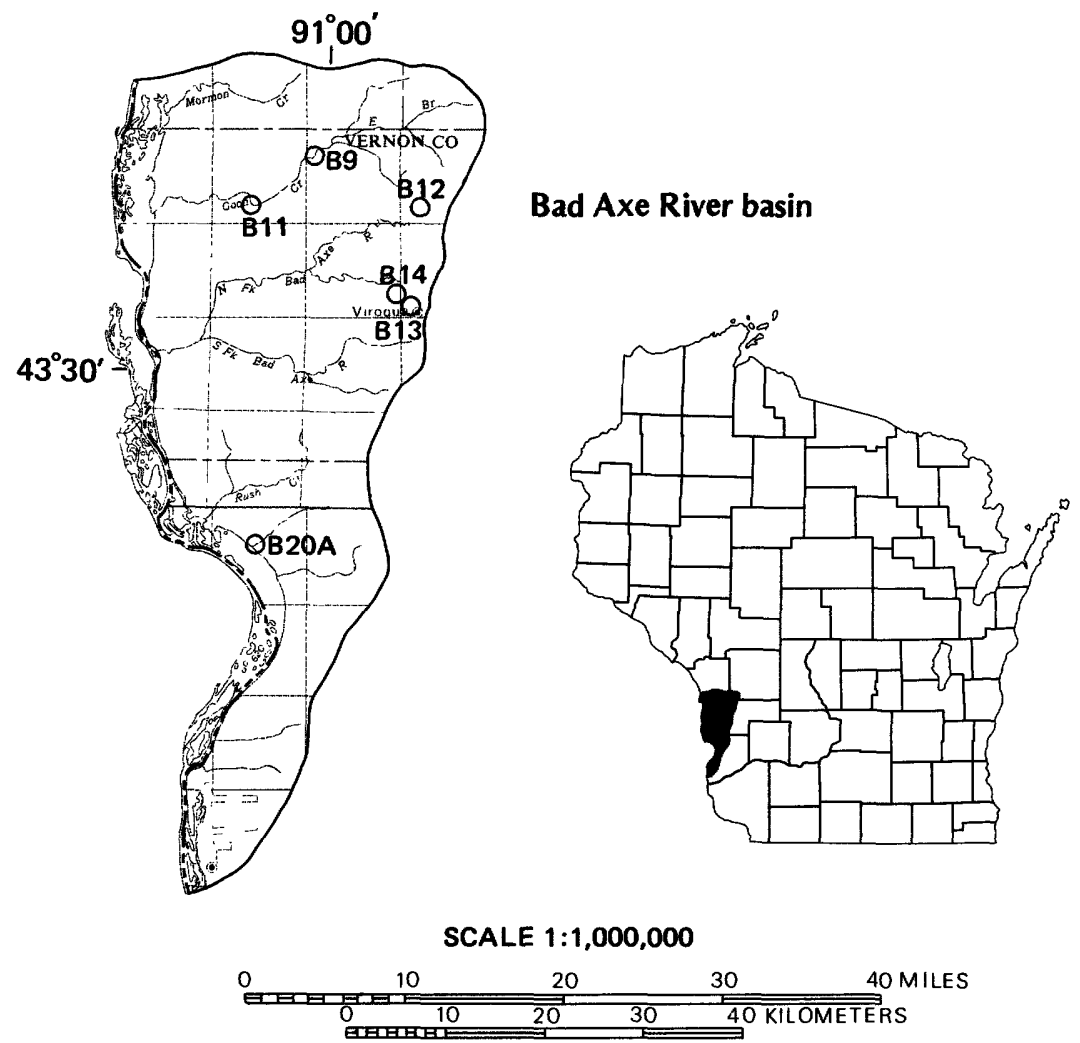

Base from U.S. Geological Survey

State base map, 1968

\begin{tabular}{|c|c|c|c|c|c|c|c|c|}
\hline $\begin{array}{l}\text { Station } \\
\text { number }\end{array}$ & 1 Stream & Station location & $\begin{array}{l}\text { Drainage } \\
\text { area } \\
\left(\mathrm{mi}^{2}\right)\end{array}$ & Date & $\begin{array}{c}\text { Discharge } \\
\left(\mathrm{ft}^{3} / \mathrm{s}\right)\end{array}$ & $\begin{array}{l}\mathrm{Q}_{7,2} \\
\left(\mathrm{ft}^{3} / \mathrm{s}\right)\end{array}$ & $\begin{array}{r}Q_{7,10} \\
\left(\mathrm{ft}^{3} / \mathrm{s}\right)\end{array}$ & Accuracy \\
\hline B9 & Coon Creek & $\begin{array}{l}\text { SW } 1 / 4 \text { NE } 1 / 4 \text { sec. } 7, \text { T. } 14 \text { N., R. } 5 \text { W., } \\
\text { Vernon County, } 700 \mathrm{ft} \text { upstream } \\
\text { from U.S. Highway } 14 \text { and } 61 \text {, at } \\
\text { Coon Valley. Wis. }\end{array}$ & 78.6 & $\begin{array}{l}\text { Aug. } 12,1970 \\
\text { June } 20,1972 \\
\text { June } 28,1973 \\
\text { Sept. } 11,1973 \\
\text { Aug. } 17,1976 \\
\text { Aug. } 9,1977 \\
\text { Sept. } 13,1977 \\
\text { Sept. } 11,1978\end{array}$ & $\begin{array}{l}34.2 \\
40.9 \\
75.8 \\
62.6 \\
46.6 \\
46.0 \\
39.3 \\
44.6\end{array}$ & 28 & 24 & $\mathbf{a}$ \\
\hline B11 & $\begin{array}{l}\text { Coon Creek } \\
\text { tributary }\end{array}$ & $\begin{array}{l}\text { NW } 1 / 4 \text { NE } 1 / 4 \text { sec. } 33, \text { T. } 14 \text { N., R. } 6 \text { W., } \\
\text { Vernon County, } 0.3 \text { mi upstream from } \\
\text { mouth, at Chaseburg, Wis. }\end{array}$ & 1.35 & Aug. 17,1976 & ${ }^{2} 0$ & 0 & $\mathbf{0}$ & c \\
\hline & Coon Creek & $\begin{array}{l}\text { NW } 1 / 4 \text { SW1/4 sec. } 27, \text { T. } 14 \text { N., R. } 6 \text { W., } \\
\text { Vernon County, at bridge on County } \\
\text { Trunk KK, } 0.4 \text { mi eest of } \\
\text { Chaseburg, Wis. }\end{array}$ & 105 & $\begin{array}{l}\text { Aug. } 17,1976 \\
\text { Aug. } 9,1977 \\
\text { Sept. } 13,1977\end{array}$ & $\begin{array}{l}61.1 \\
59.7 \\
54.5\end{array}$ & 39 & 34 & c \\
\hline B12 & $\begin{array}{l}\text { North Fork } \\
\text { Bad Axe River }\end{array}$ & $\begin{array}{l}\text { NW1/4NW1/4 sec. } 33, \text { T. } 14 \text { N., R. } 4 \text { W., } \\
\text { Vernon County, at sewage-treatment } \\
\text { plant at west edge of Westby, Wis. }\end{array}$ & .27 & & & & & \\
\hline & $\begin{array}{l}\text { North Fork } \\
\text { Bad Axe River }\end{array}$ & $\begin{array}{l}\text { SW } 1 / 4 \text { SE } 1 / 4 \text { sec. } 29, \text { T. } 14 \text { N., R. } 4 \text { W., } \\
\text { Vernon County, at bridge on country } \\
\text { road, and } 1.6 \text { mi west of Westby, } \\
\text { Wis. }\end{array}$ & 1.77 & $\begin{array}{l}\text { June } 20,1972 \\
\text { June } 28,1973 \\
\text { Sept. } 11,1973 \\
\text { Aug. } 16,1976\end{array}$ & ${ }_{0}^{20}{ }_{0}^{13}$ & 0 & 0 & c \\
\hline
\end{tabular}


Table 1. Bad Axe River basin station locations and low-flow characteristics-Continued.

\begin{tabular}{|c|c|c|c|c|c|c|c|c|}
\hline $\begin{array}{l}\text { Station } \\
\text { number }\end{array}$ & Stream & Station location & $\begin{array}{l}\text { Drainage } \\
\text { area } \\
\left(\mathrm{mi}^{2}\right)\end{array}$ & Date & $\begin{array}{l}\text { Discharge } \\
\left(\mathrm{ft}^{3} / \mathrm{s}\right)\end{array}$ & $\begin{array}{l}Q_{7,2} Q \\
\left(\mathrm{ft}^{3} / \mathrm{s}\right)(\mathrm{ft}\end{array}$ & $\begin{array}{l}7,10 \\
\left.t^{3} / s\right)\end{array}$ & $\begin{array}{c}\text { Accuracy } \\
\text { level }\end{array}$ \\
\hline B13 & $\begin{array}{l}\text { Springville } \\
\text { Branch }\end{array}$ & $\begin{array}{l}\text { NW } 1 / 4 N E 1 / 4 \text { sec. } 31, T .13 \text { N., R. } 4 \text { W., } \\
\text { Vernon County, at end of town road, } \\
\text { and } 0.5 \text { mi north of Viroqua, Wis. }\end{array}$ & 1.15 & $\begin{array}{l}\text { June } 20,1972 \\
\text { June } 28,1973 \\
\text { Sept. 13, } 1973 \\
\text { Aug. } 16,1976\end{array}$ & $\begin{array}{l}.64 \\
.71 \\
.78 \\
.52\end{array}$ & 0.47 & 0.28 & c \\
\hline \multirow[t]{2}{*}{ B14 } & $\begin{array}{l}\text { Springville } \\
\text { Branch tributary }\end{array}$ & $\begin{array}{l}\text { NW } 1 / 4 N W 1 / 4 \text { sec. } 29, \text { T. } 13 \text { N., R. } 4 \text { W., } \\
\text { Vernon County, at sewage-treatment } \\
\text { plant for Vernon County Home, and } \\
1.6 \text { mi north of Viroqua, Wis. }\end{array}$ & .31 & June 20,1972 & $2_{0}$ & 0 & $\mathbf{0}$ & c \\
\hline & $\begin{array}{l}\text { Springville } \\
\text { Branch }\end{array}$ & $\begin{array}{l}\text { SW1 } 1 / 4 \text { SE } 1 / 4 \text { sec. } 23, T .13 \text { N., R. } 5 \text { W., } \\
\text { Vernon County, at bridge on County } \\
\text { Trunk B, in Springville, Wis. }\end{array}$ & 8.93 & $\begin{array}{l}\text { June } 20,1972 \\
\text { June } 28,1973 \\
\text { Sept. 13, 1973 } \\
\text { June } 29,1976 \\
\text { Aug. } 16,1976\end{array}$ & $\begin{array}{l}2.57 \\
6.02 \\
5.61 \\
4.54 \\
3.91\end{array}$ & 2.6 & 1.1 & c \\
\hline B20A S & Sugar Creek & $\begin{array}{l}\text { NE1/4SE } 1 / 4 \text { sec. } 16, T .10 \text { N., R. } 6 \text { W., } \\
\text { Crawford County, at bridge on State } \\
\text { Highway } 35,1.2 \text { mi sou theast of } \\
\text { Ferryville, Wis. }\end{array}$ & 24.6 & $\begin{array}{l}\text { Aug. } 11,1970 \\
\text { June } 20,1972 \\
\text { June } 28,1973 \\
\text { Sept. } 13,1973 \\
\text { June } 28,1976 \\
\text { Aug. } 17,1976\end{array}$ & $\begin{array}{l}8.40 \\
10.5 \\
17.8 \\
15.2 \\
12.8 \\
12.0\end{array}$ & 8.4 & 7.2 & c \\
\hline
\end{tabular}

\footnotetext{
1 Based on 1971 report by Wisconsin Department of Natural Resources.

${ }^{2}$ Streambed is dry.
} 
Table 2. Baraboo-Lemonweir River basin station locations and low-flow characteristics.

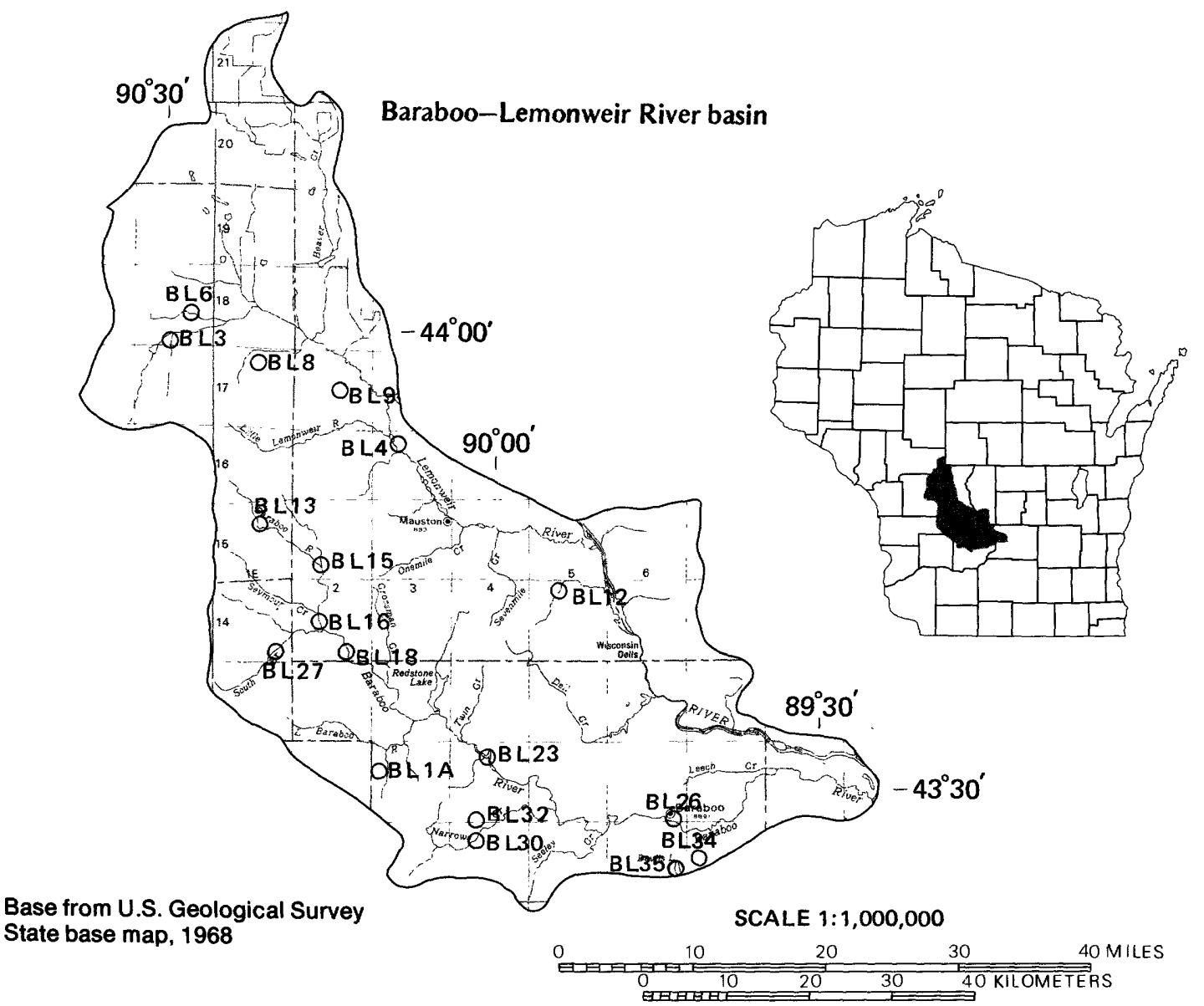

\begin{tabular}{|c|c|c|c|c|c|c|c|c|c|}
\hline $\begin{array}{l}\text { Station } \\
\text { number }\end{array}$ & Stream & Station location & $\begin{array}{l}\text { Drainage } \\
\text { area } \\
\left(\mathrm{mi}^{2}\right)\end{array}$ & Date & $\begin{array}{c}\text { Discharge } \\
\left(\mathrm{ft}^{3} / \mathrm{s}\right)\end{array}$ & $\begin{array}{l}Q_{7,2} \\
\left(\mathrm{ft}^{3} / \mathrm{s}\right)\end{array}$ & & & $\begin{array}{c}\text { Accuracy } \\
\text { level }\end{array}$ \\
\hline BL3 & $\begin{array}{l}\text { South Fork } \\
\text { Lemonweir } \\
\text { River }\end{array}$ & $\begin{array}{l}\text { NE } 1 / 4 N E 1 / 4 \text { sec. } 33, T .18 \mathrm{~N} ., \text { R. } 1 \text { W., } \\
\text { Monroe County, at bridge on town } \\
\text { road at Tomah, Wis. }\end{array}$ & 35.4 & $\begin{array}{l}\text { June } 14,1973 \\
\text { July } 26,1973 \\
\text { Oct. } 24,1973 \\
\text { June } 22,1976 \\
\text { Aug. } 11,1976\end{array}$ & $\begin{array}{l}13.1 \\
6.47 \\
12.5 \\
3.71 \\
3.58\end{array}$ & & .9 & 2.6 & $c$ \\
\hline BL4 & $\begin{array}{l}\text { Lemonweir } \\
\text { River }\end{array}$ & $\begin{array}{l}\text { SW1/4SE1/4 sec. } 8, T .16 \mathrm{~N} ., \text { R. } 3 \mathrm{E} \text {., } \\
\text { Juneau County, near center of span } \\
\text { on downstream side of bridge on } \\
\text { State Highway } 80 \text { in New Lisbon, } \\
200 \mathrm{ft} \text { downstream from recreation } \\
\text { dam, } 1.0 \text { mi upstream from Webster } \\
\text { Creek. }\end{array}$ & 507 & & & 78 & & 54 & a \\
\hline BL6 & Mud Creek & $\begin{array}{l}\text { NEY/4NW1/4 sec. } 28, T .18 \text { N., R. } 1 \text { W., } \\
\text { Monroe County, at bridge on U.S. } \\
\text { Highway } 12,2.4 \text { mi north of } \\
\text { Tomah, Wis. }\end{array}$ & 7.04 & $\begin{array}{l}\text { June } 22,1976 \\
\text { Aug. } 11,1976 \\
\text { Aug. } 30,1976 \\
\text { July } 28,1977 \\
\text { Sept. } 7,1977\end{array}$ & $\begin{array}{l}2.70 \\
2.44 \\
1.86 \\
1.48 \\
1.81\end{array}$ & & .3 & 1.6 & c \\
\hline
\end{tabular}


Table 2. Baraboo-Lemonweir River basin station locations and low-flow characteristics-Continued.

\begin{tabular}{|c|c|c|c|c|c|c|c|c|}
\hline $\begin{array}{l}\text { Station } \\
\text { number }\end{array}$ & 1 Stream & Station location & $\begin{array}{l}\text { Drainage } \\
\text { area } \\
\left(\mathrm{mi}^{2}\right)\end{array}$ & Date & $\begin{array}{c}\text { Discharge } \\
\left(\mathrm{ft}^{3} / \mathrm{s}\right)\end{array}$ & $\begin{array}{l}Q_{7,2} Q_{7} \\
\left(\mathrm{ft}^{3} / \mathrm{s}\right)\left(\mathrm{ft}^{3}\right)\end{array}$ & & $\begin{array}{l}\text { Accuracy } \\
\text { level }\end{array}$ \\
\hline \multirow[t]{2}{*}{ BL8 } & Allen Creek & $\begin{array}{l}\text { NW } 1 / 4 \text { SE } 1 / 4 \text { sec. } 10, T .17 \text { N., R. } 1 \text { E., } \\
\text { Monroe County, at sewage-treatment } \\
\text { plant, } 1.0 \text { mi east of Oakdale. Wis. }\end{array}$ & 2.58 & & & & & \\
\hline & Allen Creek & $\begin{array}{l}\text { NW } 1 / 4 \text { SW } 1 / 4 \text { sec. } 2, \text { T. } 17 \text { N., R. } 1 \text { E., } \\
\text { Monroe County, at bridge on town } \\
\text { road, } 1.9 \text { mi northeast of Oakdale, } \\
\text { Wis. }\end{array}$ & 7.44 & $\begin{array}{l}\text { June } 14,1973 \\
\text { July } 25,1973 \\
\text { Oct. } 24,1973 \\
\text { June } 22,1976 \\
\text { Aug. } 11,1976 \\
\text { Sept. } 29,1976 \\
\text { Oct. } 14,1976 \\
\text { Nov. } 1,1976 \\
\text { Nov. } 15,1976 \\
\text { Dec. } 1,1976\end{array}$ & $\begin{array}{l}6.00 \\
3.43 \\
4.34 \\
3.24 \\
1.97 \\
2.15 \\
.94 \\
2.28 \\
2.54 \\
3.77\end{array}$ & 2.5 & 2.0 & $\mathbf{c}$ \\
\hline BL9 & $\begin{array}{l}\text { Lemonweir } \\
\text { River tributary }\end{array}$ & $\begin{array}{l}\text { NW1/4NE } 1 / 4 \text { sec. } 14, T .17 \text { N., R. } 2 \text { E., } \\
\text { Juneau County, at bridge on town } \\
\text { road, } 2.5 \text { mi northeast of Camp } \\
\text { Williams, Wis. }\end{array}$ & 14.1 & $\begin{array}{l}\text { June } 14,1973 \\
\text { July } 25,1973 \\
\text { Oct. } 24,1973 \\
\text { June } 22,1976 \\
\text { Aug. } 11,1976\end{array}$ & $\begin{array}{l}13.7 \\
6.75 \\
6.90 \\
5.00 \\
2.98\end{array}$ & 3.5 & 2.7 & c \\
\hline BL12 & Lyndon Creek & $\begin{array}{l}\text { SW } 1 / 4 \text { NW } 1 / 4 \text { sec. } 10, \text { T. } 14 \text { N., R. } 5 \text { E., } \\
\text { Juneau County, at bridge on U.S. } \\
\text { Highways } 12 \text { and } 16 \text {, at Lyndon } \\
\text { Station, Wis. }\end{array}$ & 6.10 & $\begin{array}{l}\text { June } 22,1976 \\
\text { Aug. } 9,1976 \\
\text { Aug. } 30,1976 \\
\text { July } 28,1977 \\
\text { Sept. } 7,1977\end{array}$ & $\begin{array}{l}1.30 \\
1.27 \\
1.17 \\
1.24 \\
1.10\end{array}$ & 1.0 & .76 & c \\
\hline BL13 & Baraboo River & $\begin{array}{l}\text { NE } 1 / 4 \text { SW } 1 / 4 \text { sec. } 10, \text { T. } 15 \text { N., R. } 1 \text { E., } \\
\text { Monroe County, at bridge on State } \\
\text { Highway } 71 \text {, at Kendall, Wis. }\end{array}$ & 15.9 & $\begin{array}{l}\text { June } 13,1973 \\
\text { July } 25,1973 \\
\text { Oct. } 24,1973 \\
\text { June } 22,1976 \\
\text { Aug. } 10,1976\end{array}$ & $\begin{array}{l}10.7 \\
7.96 \\
6.38 \\
2.74 \\
3.47\end{array}$ & 2.4 & 1.7 & c \\
\hline BL15 & Baraboo River & $\begin{array}{l}\text { NW1/4SW1/4 sec. } 33, T .15 \text { N., R. } 2 \text { E., } \\
\text { Juneau County, at bridge on County } \\
\text { Trunk O, at Elroy, Wis. }\end{array}$ & 59.4 & $\begin{array}{l}\text { June } 14,1973 \\
\text { Oct. 24, } 1973 \\
\text { June } 22,1976 \\
\text { Aug. } 10,1976\end{array}$ & $\begin{array}{l}37.9 \\
27.0 \\
19.4 \\
16.3\end{array}$ & 12 & 9.1 & c \\
\hline BL16 & Baraboo River & $\begin{array}{l}\text { SE } 1 / 4 N W 1 / 4 \text { sec. } 21, \text { T. } 14 \text { N., R. } 2 \text { E.., } \\
\text { Juneau County, at sewage-treatment } \\
\text { plant, at Union Center, Wis. }\end{array}$ & 97.8 & $\begin{array}{l}\text { June } 23,1976 \\
\text { Aug. } 10,1976 \\
\text { Sept. } 7,1977\end{array}$ & $\begin{array}{l}27.6 \\
29.6 \\
21.6\end{array}$ & 19 & 15 & c \\
\hline BL18 & Baraboo River & $\begin{array}{l}\text { SW } 1 / 4 N W 1 / 4 \text { sec. } 35, T .14 \text { N., R. } 2 \text { E., } \\
\text { Juneau County, at bridge on town } \\
\text { road, at Wonewoc, Wis. }\end{array}$ & 174 & $\begin{array}{l}\text { July } 25,1973 \\
\text { June } 23,1976 \\
\text { Aug. } 10,1976 \\
\text { Sept. } 7,1977\end{array}$ & $\begin{array}{l}96.8 \\
49.0 \\
46.5 \\
40.4\end{array}$ & 32 & 21 & c \\
\hline BL23 & Baraboo River & $\begin{array}{l}\text { NE } 1 / 4 \text { NE } 1 / 4 \text { sec. } 9, \text { T. } 12 \text { N., R. } 4 \text { E., } \\
\text { Sauk County, } 300 \text { ft upstream from } \\
\text { Hay Creek, at Reedsburg. Wis. }\end{array}$ & 376 & $\begin{array}{l}\text { June } 21,1976 \\
\text { Aug. 10, } 1976 \\
\text { July } 29,1977 \\
\text { Sept. } 7,1977 \\
\text { Sept. } 5,1978\end{array}$ & $\begin{array}{c}114 \\
90.9 \\
206 \\
70.0 \\
97.2\end{array}$ & 67 & 49 & c \\
\hline \multirow[t]{2}{*}{ BL26 } & $\begin{array}{l}\text { Baraboo River } \\
\text { tributary }\end{array}$ & $\begin{array}{l}\text { NE } 1 / 4 \text { SW } 1 / 4 \text { sec. } 12, \text { T. } 11 \text { N., R. } 6 \text { E., } \\
\text { Sauk County, at bridge on town road, } \\
1.8 \text { mi south of Baraboo, Wis. }\end{array}$ & 6.55 & June 13,1973 & ${ }^{2} 0$ & 0 & 0 & c \\
\hline & $\begin{array}{l}\text { Baraboo River } \\
\text { tributary }\end{array}$ & $\begin{array}{l}\text { SE } 1 / 4 \text { SW } 1 / 4 \text { sec. } 1, \text { T. } 11 \text { N., R. } 6 \text { E., } \\
\text { Sauk County, at culvert on town } \\
\text { road, at Baraboo, Wis. }\end{array}$ & 8.00 & $\begin{array}{l}\text { June } 13,1973 \\
\text { July } 24,1973 \\
\text { Oct. } 25,1973 \\
\text { June } 21,1976 \\
\text { Aug. } 9,1976\end{array}$ & $\begin{array}{l}.37 \\
.16 \\
.41 \\
.27 \\
.14\end{array}$ & .11 & .06 & c \\
\hline BL27 & $\begin{array}{l}\text { South Branch } \\
\text { Baraboo River }\end{array}$ & $\begin{array}{l}\text { NE } 1 / 4 N^{1} 1 / 4 \text { sec. } 35, \text { T. } 14 \text { N., R. } 1 \text { E., } \\
\text { Vernon County, at bridge on County } \\
\text { Trunk FF, at Hilisboro, Wis. }\end{array}$ & 39.1 & $\begin{array}{ll}\text { Dec. } & 8,1970 \\
\text { June } 14,1973 \\
\text { July } 25,1973 \\
\text { Oct. } 24,1973 \\
\text { June } 23,1976 \\
\text { Aug. } 10,1976\end{array}$ & $\begin{array}{l}10.6 \\
31.3 \\
26.1 \\
22.7 \\
9.86 \\
9.40\end{array}$ & 5.2 & 3.1 & c \\
\hline
\end{tabular}


Table 2. Baraboo-Lemonweir River basin station locations and low-flow characteristics-Continued.

\begin{tabular}{|c|c|c|c|c|c|c|c|c|}
\hline $\begin{array}{l}\text { Station } \\
\text { number }\end{array}$ & Stream & Station location & $\begin{array}{l}\text { Drainage } \\
\text { area } \\
\left(m i^{2}\right)\end{array}$ & Date & $\begin{array}{c}\text { Discharge } \\
\left(\mathrm{ft}^{3} / \mathrm{s}\right)\end{array}$ & $\begin{array}{l}Q_{7,2} Q_{7} \\
\left(\mathrm{ft}^{3} / \mathrm{s}\right) \\
\left(\mathrm{ft}^{3}\right.\end{array}$ & $3 / s\rangle$ & $\begin{array}{c}\text { Accuracy } \\
\text { level }\end{array}$ \\
\hline BL30 & Narrows Creek & $\begin{array}{l}\text { NE } 1 / 4 \text { SE } 1 / 4 \text { sec. } 8 \text {, T. } 11 \text { N., R. } 4 \text { E., } \\
\text { Sauk County, at bridge on State } \\
\text { Highways } 23 \text { and } 154,0.2 \text { mi } \\
\text { north of Loganville, Wis. }\end{array}$ & 40.1 & $\begin{array}{l}\text { Aug. } 20,1969 \\
\text { July } 9,1970 \\
\text { Aug. } 18,1970 \\
\text { June } 13,1973 \\
\text { July } 24,1973 \\
\text { Oct. } 25,1973 \\
\text { June } 21,1976 \\
\text { Aug. } 10,1976\end{array}$ & $\begin{array}{l}36.49 \\
6.56 \\
4.97 \\
23.2 \\
14.8 \\
13.4 \\
6.30 \\
6.61\end{array}$ & 3.6 & 2.7 & b \\
\hline BL32 & $\begin{array}{l}\text { Narrows Creek } \\
\text { tributary }\end{array}$ & $\begin{array}{l}\text { NE } 1 / 4 \text { SW } 1 / 4 \text { sec. } 34, \text { T. } 12 \text { N., R. } 4 \text { E., } \\
\text { Sauk County, at bridge on State } \\
\text { Highway } 154,2.8 \text { mi northeast } \\
\text { of Loganville, Wis. }\end{array}$ & 4.57 & $\begin{array}{l}\text { June } 13,1973 \\
\text { July } 24,1973 \\
\text { Oct. } 25,1973 \\
\text { June } 21,1976 \\
\text { Aug. } 10,1976\end{array}$ & $\begin{array}{c}2.19 \\
1.54 \\
1.72 \\
.89 \\
1.21\end{array}$ & .74 & .58 & c \\
\hline BL34 & $\begin{array}{l}\text { Wisconsin River } \\
\text { tributary }\end{array}$ & $\begin{array}{l}\text { SW\% } / 4 \text { SW } \% \text { sec. } 23, \text { T. } 11 \text { N., R. } 7 \text { E., } \\
\text { Sauk County, at bridge on country } \\
\text { road, } 2.6 \text { mi north of Merrimac, Wis. }\end{array}$ & 2.82 & $\begin{array}{l}\text { June } 21,1976 \\
\text { Aug. } 9,1976 \\
\text { Aug. } 30,1976 \\
\text { July } 28,1977 \\
\text { Sept. } 7,1977\end{array}$ & $\begin{array}{l}.68 \\
.37 \\
.52 \\
.27 \\
.30\end{array}$ & .17 & .09 & c \\
\hline BL35 & $\begin{array}{l}\text { Wisconsin River } \\
\text { tributary }\end{array}$ & $\begin{array}{l}\text { NW/ } / 4 \text { SE } 1 / \text { sec. } 28, \text { T. } 11 \text { N., R. } 7 \text { E., } \\
\text { Sauk County, at bridge on State } \\
\text { Highway } 113,3.0 \text { mi northwest of } \\
\text { Merrimac, Wis. }\end{array}$ & 3.16 & $\begin{array}{l}\text { June } 13,1973 \\
\text { July } 24,1973 \\
\text { Oct. } 25,1973 \\
\text { June } 21,1976 \\
\text { Aug. } 9,1976\end{array}$ & $\begin{array}{l}3.50 \\
4.30 \\
2.17 \\
.71 \\
1.00\end{array}$ & .35 & .21 & c \\
\hline BL1A & McGlynn Creek & $\begin{array}{l}\text { NW/ NW\% sec. } 8, \text { T. } 12 \text { N., R. } 3 \text { E., } \\
\text { Sauk County, } 0.2 \text { mi upstream from } \\
\text { bridge on State Highway } 58,1.6 \mathrm{mi} \\
\text { northeast of Cazenovia, Wis. }\end{array}$ & & $\begin{array}{l}\text { June } 23,1976 \\
\text { Aug. } 9,1976 \\
\text { Aug. } 30,1976 \\
\text { July } 28,1977 \\
\text { Sept. } 7,1977\end{array}$ & $\begin{array}{l}7.39 \\
5.90 \\
5.16 \\
3.99 \\
4.30\end{array}$ & 3.3 & 2.4 & c \\
\hline
\end{tabular}

\footnotetext{
1 Based on 1973 report by Wisconsin Department of Natural Resources (DNR). Site lettered "A" was not included in 1973 DNR report.

2 Streambed is dry.

${ }^{3}$ Additional discharge meesurements are available.
} 
Table 3. Black River basin station location and low-flow characteristics.
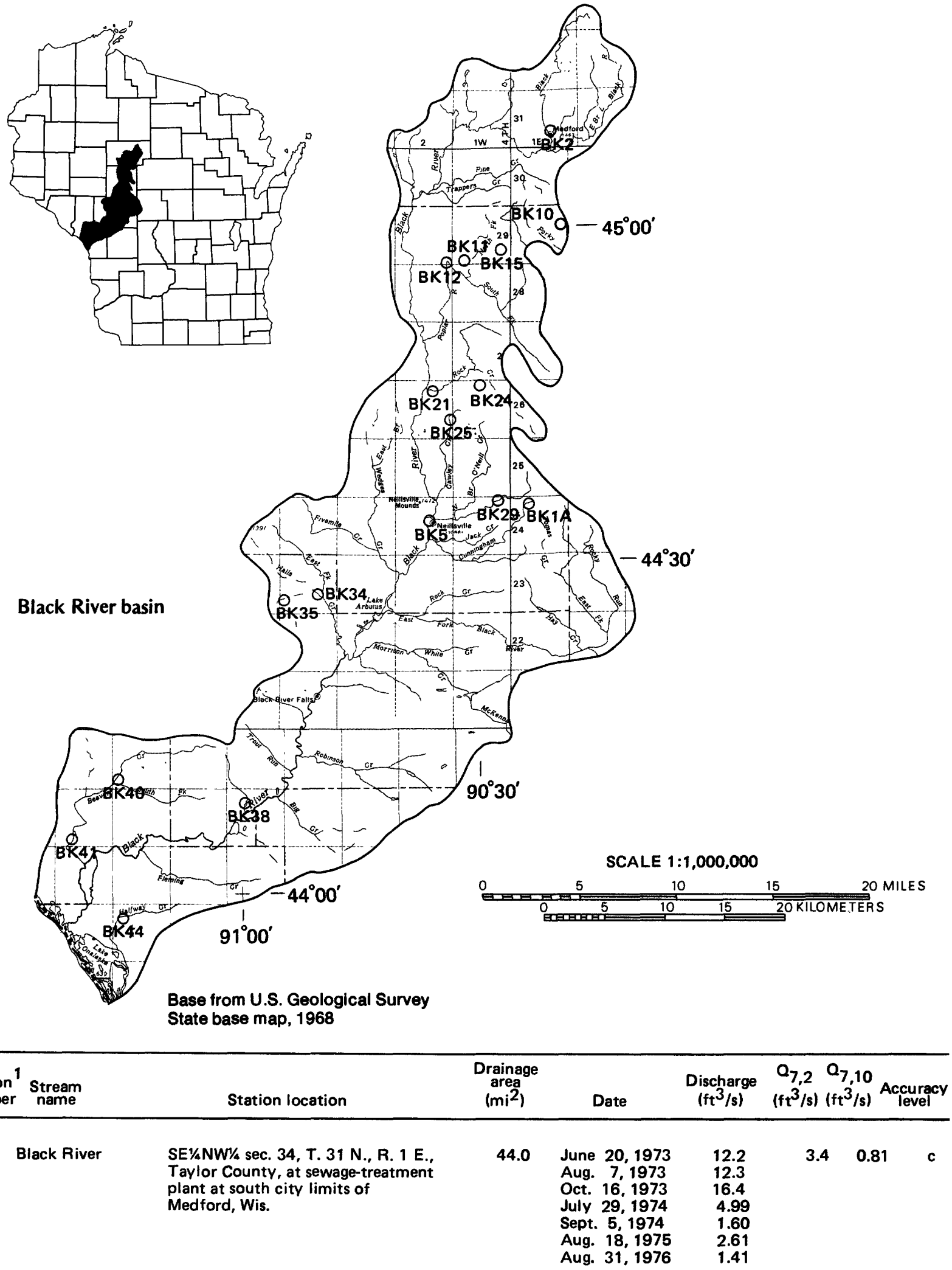
Table 3. Black River basin station location and low-flow characteristics-Continued.

\begin{tabular}{|c|c|c|c|c|c|c|c|c|}
\hline $\begin{array}{l}\text { Station } \\
\text { number }\end{array}$ & 1 Stream & Station location & $\begin{array}{l}\text { Drainage } \\
\text { area } \\
\left(\mathrm{mi}^{2}\right)\end{array}$ & Date & $\begin{array}{l}\text { Discharge } \\
\left(\mathrm{ft}^{3} / \mathrm{s}\right)\end{array}$ & $\begin{array}{l}\mathrm{a}_{7,2} \mathrm{a}_{7,1} \\
\left(\mathrm{ft}^{3} / \mathrm{s}\right)\left(\mathrm{ft}^{3}\right)\end{array}$ & $10 \mathrm{Ac}$ & $\begin{array}{l}\text { Accuracy } \\
\text { level }\end{array}$ \\
\hline BK5 & Black River & $\begin{array}{l}\text { NWY } 1 / 4 \text { SW } 1 / 4 \text { sec. } 15, \text { T. } 24 \text { N., R. } 2 \text { W., } \\
\text { Clark County, on right bank at } \\
\text { downstream side of bridge on U.S. } \\
\text { Highway } 10 \text { in Neillsville, } 1.0 \mathrm{mi} \\
\text { downstream from O'Neill Creek. }\end{array}$ & 756 & & & 26 & 6.6 & $\mathbf{a}$ \\
\hline BK10 & $\begin{array}{l}\text { North Fork } \\
\text { Poplar River }\end{array}$ & $\begin{array}{l}\text { NE1/4NE1/4 sec. } 14, T .29 \text { N., R. } 1 \text { E., } \\
\text { Clark County, at sewage-treatment } \\
\text { plant, at Dorchester, Wis. }\end{array}$ & 2.37 & $\begin{array}{lc}\text { June } & 20,1973 \\
\text { Aug. } & 7,1973 \\
\text { Oct. } 16,1973 \\
\text { July } 29,1974 \\
\text { Sept. } 5,1974 \\
\text { Aug. } 18,1975\end{array}$ & $\begin{array}{l}.08 \\
.04 \\
4^{.01} \\
4_{0}^{0} \\
4_{0}^{0}\end{array}$ & 0 & 0 & c \\
\hline BK11 & $\begin{array}{l}\text { North Fork } \\
\text { Poplar River }\end{array}$ & $\begin{array}{l}\text { NE1/4NE1/4 sec. } 5, \text { T. } 28 \text { N., R. } 1 \text { W., } \\
\text { Clark County, at bridge on State } \\
\text { Highway } 29,2.1 \text { mi east of } \\
\text { Owen, Wis. }\end{array}$ & 55.6 & $\begin{array}{lc}\text { Oct. } & 24,1968 \\
\text { Aug. } 27,1969 \\
\text { June } 20,1973 \\
\text { Aug. } 8,1973 \\
\text { Oct. } 16,1973 \\
\text { July } 30,1974 \\
\text { Sept. } 5,1974 \\
\text { Aug. } 18,1975 \\
\text { Aug. } 31,1976\end{array}$ & $\begin{array}{l}6.31 \\
.62 \\
8.02 \\
9.21 \\
2.61 \\
.70 \\
.46 \\
.10 \\
.15\end{array}$ & .49 & .07 & c \\
\hline BK12 $F$ & Poplar River & $\begin{array}{l}\text { NE1/4NW1/4 sec. 1, T. } 28 \text { N., R. } 2 \text { W., } \\
\text { Clark County, at sewage-treatment } \\
\text { plant, at Owen, Wis. }\end{array}$ & 145 & $\begin{array}{lc}\text { June } & 20,1973 \\
\text { Aug. } & 7,1973 \\
\text { Oct. } & 16,1973 \\
\text { July } & 30,1974 \\
\text { Sept. } 5,1974 \\
\text { Aug. } 18,1975 \\
\text { Aug. } & 31,1976\end{array}$ & $\begin{array}{l}22.3 \\
15.7 \\
5.07 \\
1.99 \\
.21 \\
1.39 \\
.53\end{array}$ & 1.5 & .28 & c \\
\hline BK15 & $\begin{array}{l}\text { North Fork } \\
\text { Poplar River } \\
\text { tributary }\end{array}$ & $\begin{array}{l}\text { NWY/4NE1/4 sec. } 36, T .29 \text { N., R. } 1 \text { W., } \\
\text { Clark County, at country road bridge, } \\
0.5 \text { mi northwest of Curtiss, Wis. }\end{array}$ & .66 & $\begin{array}{l}\text { June } 20,1973 \\
\text { Aug. } 7,1973 \\
\text { Oct. } 6,1973 \\
\text { July } 30,1974 \\
\text { Sept. } 5,1974 \\
\text { Aug. } 18,1975\end{array}$ & $\begin{array}{l}2 \\
2_{0}^{0} \\
20 \\
20 \\
20 \\
2_{0}^{0}\end{array}$ & 0 & 0 & c \\
\hline BK21 & Rock Creek & $\begin{array}{l}\text { SE } 1 / 4 \text { SE } 1 / 4 \text { sec. } 3, \text { T. } 26 \text { N., R. } 2 \text { W., } \\
\text { Clark County, at bridge on State } \\
\text { Highway } 73,0.5 \text { mi south of } \\
\text { Greenwood, Wis. }\end{array}$ & 78.4 & $\begin{array}{lc}\text { Oct. } & 25,1968 \\
\text { Aug. } 28,1969 \\
\text { Aug. } 11,1970 \\
\text { Aug. } 8,1972 \\
\text { June } 20,1973 \\
\text { Aug. } 8,1973 \\
\text { Oct. } 16,1973 \\
\text { July } 30,1974 \\
\text { Sept. } 5,1974 \\
\text { Aug. } 19,1975 \\
\text { Aug. } 31,1976\end{array}$ & $\begin{array}{l}7.38 \\
1.74 \\
1.19 \\
24.0 \\
13.6 \\
10.4 \\
4.67 \\
1.22 \\
1.58 \\
.99 \\
.16\end{array}$ & .88 & .12 & c \\
\hline BK24 & Bear Creek & $\begin{array}{l}\text { SW1/4SW1/4 sec. 10, T. } 26 \text { N., R. } 1 \text { W., } \\
\text { Clark County, at bridge on County } \\
\text { Trunk K, upstream from sewage- } \\
\text { treatment plant, at Loyal, Wis. }\end{array}$ & 2.97 & $\begin{array}{lc}\text { Aug. } & 8,1972 \\
\text { June } & 20,1973 \\
\text { Aug. } & 8,1973 \\
\text { Oct. } 16,1973 \\
\text { July } 30,1974 \\
\text { Sept. } 5,1974 \\
\text { Aug. } 19,1975 \\
\text { Aug. } 31,1976\end{array}$ & $\begin{array}{l}.95 \\
.37 \\
3.36 \\
.12 \\
4.14 \\
{ }^{4} 0 \\
.04\end{array}$ & .08 & .03 & c \\
\hline BK25 & Cauley Creek & $\begin{array}{l}\text { SE } 1 / 4 S^{1} 1 / 4 \text { sec. } 24, T .26 \text { N., R. } 2 \text { W., } \\
\text { Clark County, at bridge on town } \\
\text { road, } 3.7 \text { mi southwest of Loyal, Wis. }\end{array}$ & 5.14 & $\begin{array}{l}\text { Sept. } 5,1974 \\
\text { Aug. } 19,1975 \\
\text { Aug. } 23,1977\end{array}$ & $\begin{array}{l}.20 \\
.15 \\
.12\end{array}$ & .11 & .02 & c \\
\hline
\end{tabular}


Table 3. Black River basin station location and low-flow characteristics-Continued.

\begin{tabular}{|c|c|c|c|c|c|c|c|c|}
\hline $\begin{array}{l}\text { Station } \\
\text { number }\end{array}$ & ${ }^{1} \underset{\text { name }}{\text { Stream }}$ & Station location & $\begin{array}{c}\text { Drainage } \\
\text { area } \\
\left(\mathrm{mi}^{2}\right)\end{array}$ & Date & $\begin{array}{l}\text { Discharge } \\
\left(\mathrm{ft}^{3} / \mathrm{s}\right)\end{array}$ & $\begin{array}{l}\mathrm{Q}_{7,2} \quad \mathrm{Q}_{7} \\
\left(\mathrm{ft}^{3} / \mathrm{s}\right)\left(\mathrm{ft}^{3}\right.\end{array}$ & $3 / 5)$ & $\underset{\text { Aevel }}{\text { Accuracy }}$ \\
\hline BK29 & $\begin{array}{l}\text { South Branch } \\
\text { O'Neill Creek }\end{array}$ & $\begin{array}{l}\text { NW } 1 / 4 S E 1 / 4 \text { sec. } 2, T .24 \text { N., R. } 1 \text { W., } \\
\text { Clark County, at sewage-treatment } \\
\text { plant, } 0.2 \text { mi downstream from } \\
\text { County Trunk K bridge, at } \\
\text { Granton, Wis. }\end{array}$ & 16.9 & $\begin{array}{l}\text { Aug. } 7,1972 \\
\text { June } 20,1973 \\
\text { Aug. } 8,1973 \\
\text { Oct. } 16,1973 \\
\text { July } 31,1974 \\
\text { Sept. } 5,1974 \\
\text { Aug. } 19,1975 \\
\text { Aug. } 31,1976 \\
\text { July } 28,1977 \\
\text { Aug. } 18,1977\end{array}$ & $\begin{array}{l}1.61 \\
2.70 \\
3.80 \\
1.37 \\
.25 \\
.64 \\
.28 \\
.17 \\
1.08 \\
.28\end{array}$ & 0.30 & 0.07 & c \\
\hline BK34 & Halls Creek & $\begin{array}{l}\text { SE } 1 / 4 N W 1 / 4 \text { sec. } 26, T .23 \text { N., R. } 4 \text { W., } \\
\text { Jackson County, at sewage-treatment } \\
\text { plant, } 0.3 \text { mi downstream from } \\
\text { U. S. Highway } 12 \text { bridge, at } \\
\text { Merrillan, Wis. }\end{array}$ & 46.3 & $\begin{array}{l}\text { Aug. } 7,1972 \\
\text { June } 20,1973 \\
\text { Aug. } 8,1973 \\
\text { Oct. } 15,1973 \\
\text { July } 31,1974 \\
\text { Sept. } 6,1974 \\
\text { Aug. } 19,1975 \\
\text { Sept. } 1,1976\end{array}$ & $\begin{array}{l}21.2 \\
44.3 \\
97.3 \\
27.8 \\
16.7 \\
19.1 \\
16.8 \\
22.0\end{array}$ & 12 & 8.4 & c \\
\hline BK35 & $\begin{array}{l}\text { South Fork } \\
\text { Halls Creek }\end{array}$ & $\begin{array}{l}\text { SE } 1 / 4 \text { SW } 1 / 4 \text { sec. } 30, \text { T. } 22 \text { N., R. } 4 \text { W., } \\
\text { Jackson County, at bridge on County } \\
\text { Trunk F, } 0.1 \text { mi above sewage- } \\
\text { treatment plant, at Alma Center, Wis. }\end{array}$ & 6.16 & $\begin{array}{l}\text { Aug. } 7,1972 \\
\text { June } 20,1973 \\
\text { Aug. } 8,1973 \\
\text { Oct. } 15,1973 \\
\text { July } 31,1974 \\
\text { Sept. } 6,1974 \\
\text { Aug. } 19,1975 \\
\text { Sept. } 1,1976\end{array}$ & $\begin{array}{c}3.56 \\
7.65 \\
18.7 \\
5.55 \\
2.98 \\
3.34 \\
2.35 \\
1.18\end{array}$ & 1.3 & .80 & c \\
\hline BK38 & Douglas Creek & $\begin{array}{l}\text { SW1/4SW1/4 sec. 9, T. } 19 \text { N., R. } 5 \text { W., } \\
\text { Jackson County, at bridge on County } \\
\text { Trunk E, just upstream from } \\
\text { sewage-treatment plant, at } \\
\text { Melrose, Wis. }\end{array}$ & 22.9 & $\begin{array}{lc}\text { Aug. } & 12,1970 \\
\text { Aug. } & 9,1972 \\
\text { June } & 21,1973 \\
\text { Aug. } & 9,1973 \\
\text { Oct. } 15,1973 \\
\text { July } 31,1974 \\
\text { Sept. } 4,1974 \\
\text { Aug. } 19,1975 \\
\text { Sept. } 1,1976\end{array}$ & $\begin{array}{c}9.01 \\
12.4 \\
20.1 \\
24.0 \\
18.5 \\
13.3 \\
17.1 \\
9.82 \\
11.4\end{array}$ & 7.8 & 5.6 & c \\
\hline BK40 & Beaver Creek & $\begin{array}{l}\text { SW1/4NW1/4 sec. } 31, T .20 \text { N., R. } 7 \text { W., } \\
\text { Trempealeau County, at bridge on } \\
\text { County Trunk C, } 0.3 \text { mi upstream } \\
\text { from confluence with South Fork } \\
\text { Beaver Creek, at Ettrick, Wis. }\end{array}$ & 51.0 & $\begin{array}{l}\text { Aug. } 10,1970 \\
\text { Aug. } 21,1970 \\
\text { Aug. } 9,1973 \\
\text { Oct. } 15,1973 \\
\text { July } 31,1974 \\
\text { Sept. } 4,1974 \\
\text { Aug. } 19,1975 \\
\text { Sept. } 1,1976\end{array}$ & $\begin{array}{l}17.4 \\
17.6 \\
93.9 \\
39.4 \\
30.1 \\
30.5 \\
25.6 \\
23.4\end{array}$ & 14 & 9.2 & c \\
\hline BK41 & Beaver Creek & $\begin{array}{l}\text { SW1/4NE } 1 / 4 \text { sec. } 32, \mathrm{~T} .19 \mathrm{~N} ., \mathrm{R} .8 \mathrm{~W} . \text {, } \\
\text { Trempealeau County, at sewage- } \\
\text { treatment plant, about } 0.4 \mathrm{mi} \\
\text { upstream from State Highway } 54 \\
\text { bridge, at Galesville, Wis. }\end{array}$ & 147 & $\begin{array}{l}\text { Aug. } 9,1972 \\
\text { June } 21,1973 \\
\text { Aug. } 9,1973 \\
\text { Oct. } 15,1973 \\
\text { July } 31,1974 \\
\text { Sept. } 4,1974 \\
\text { Aug. } 20,1975 \\
\text { Sept. } 2,1976\end{array}$ & $\begin{array}{c}72.4 \\
107 \\
227 \\
92.4 \\
69.6 \\
87.9 \\
63.9 \\
53.0\end{array}$ & 41 & 29 & c \\
\hline BK44 & Halfway Creek & $\begin{array}{l}\text { NW1/4SE1/4 sec. } 7, T .17 \mathrm{~N} ., \mathrm{R} .7 \mathrm{~W} . \text {, } \\
\text { La Crosse County, at sewage- } \\
\text { treatment plant, } 0.4 \text { mi downstream } \\
\text { from County Trunk DH, in } \\
\text { Holmen, Wis. }\end{array}$ & 31.1 & $\begin{array}{l}\text { Aug. } 9,1972 \\
\text { June } 21,1973 \\
\text { Aug. } 9,1973 \\
\text { Oct. 15, 1973 } \\
\text { July } 31,1974 \\
\text { Sept. } 4,1974 \\
\text { Aug. 20, } 1975 \\
\text { Sept. 2, } 1976 \\
\text { July } 21,1977\end{array}$ & $\begin{array}{c}7.31 \\
14.4 \\
24.0 \\
18.8 \\
9.38 \\
11.2 \\
11.9 \\
7.96 \\
7.48\end{array}$ & 5.1 & 3.1 & c \\
\hline
\end{tabular}


Table 3. Black River basin station location and low-flow characteristics-Continued.

\begin{tabular}{|c|c|c|c|c|c|c|}
\hline 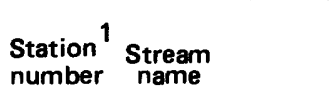 & Station location & $\begin{array}{l}\text { Drainage } \\
\text { area } \\
\left(\mathrm{mi}^{2}\right)\end{array}$ & Date & $\begin{array}{c}\text { Discharge } \\
\left(\mathrm{ft}^{3} / \mathrm{s}\right)\end{array}$ & $\begin{array}{l}\mathrm{Q}_{7,2} \\
\left(\mathrm{ft}^{3} / \mathrm{s}\right)\left(\mathrm{ft}^{3} / \mathrm{s}\right)\end{array}$ & $\begin{array}{c}\text { Accuracy } \\
\text { level }\end{array}$ \\
\hline BK1A Cunningham Creek & $\begin{array}{l}\text { SE1/4SWY/4 sec. } 5, \text { T. } 24 \text { N., R. } 1 \text { E., } \\
\text { Clark County, at bridge on U.S. } \\
\text { Highway } 10 \text {, at Lynn, Wis. }\end{array}$ & 2.62 & $\begin{array}{l}\text { Aug. } 19,1976 \\
\text { Aug. } 31,1976 \\
\text { Aug. } 23,1977\end{array}$ & $\begin{array}{r}0.06 \\
.08 \\
.06\end{array}$ & $0.08 \quad 0.02$ & c \\
\hline
\end{tabular}

1 Based on 1969 report by Wisconsin Department of Natural Resources (DNR). Site lettered "A" was not included in 1969 DNR report.

2 Streambed is dry.

${ }^{4}$ Negligible discherge--water ponded, unable to measure velocity. 
Table 4. Lower Chippewa River basin station locations and low-flow characteristics.

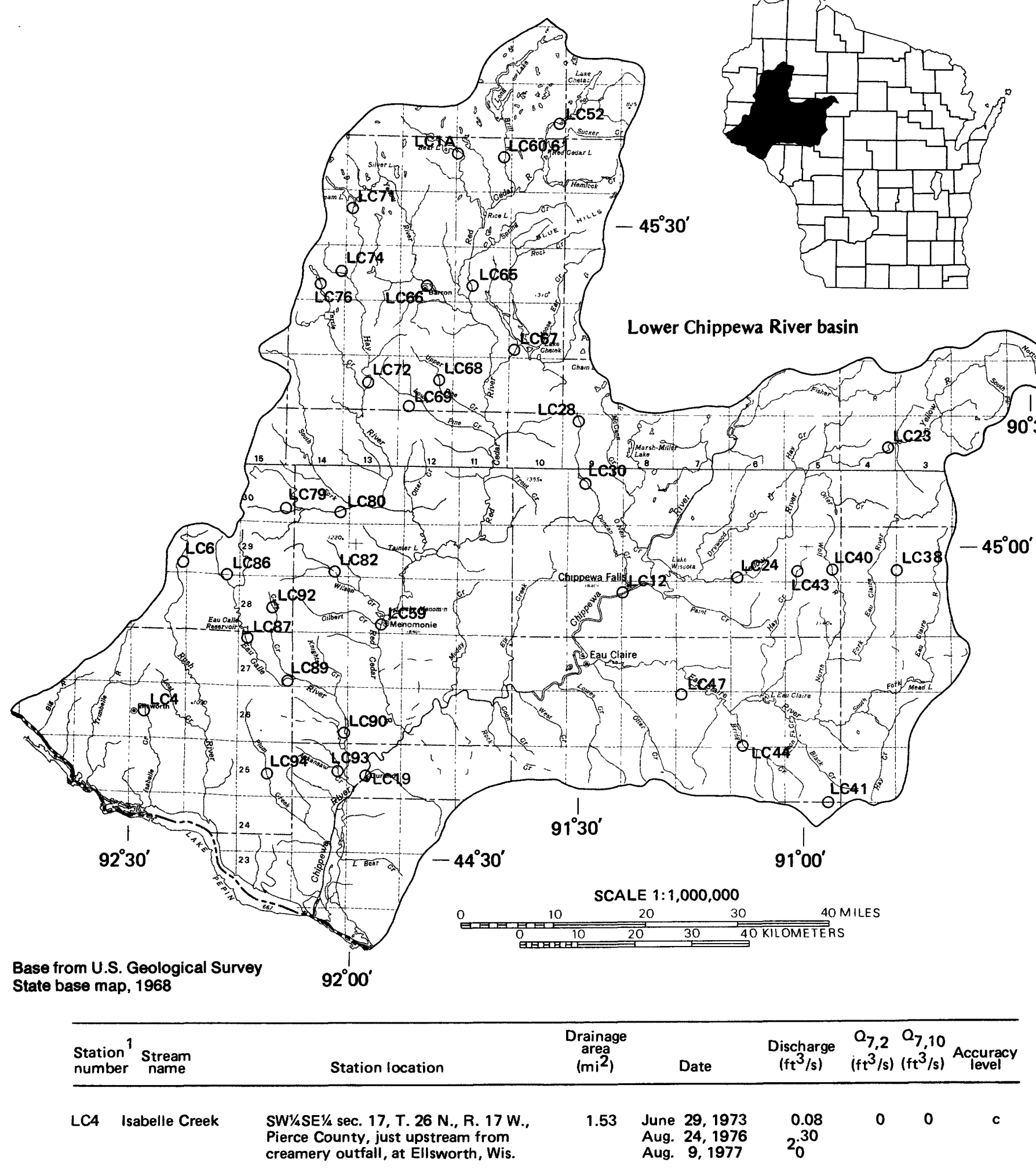


Table 4. Lower Chippewa River basin station locations and low-flow characteristics-Continued.

\begin{tabular}{|c|c|c|c|c|c|c|c|c|}
\hline $\begin{array}{l}\text { Station } \\
\text { numbe }\end{array}$ & er $^{1} \underset{\text { name }}{\text { Stream }}$ & Station location & $\begin{array}{l}\text { Drainage } \\
\text { area } \\
\left(\mathrm{mi}^{2}\right)\end{array}$ & Date & $\begin{array}{c}\text { Discharge } \\
\left(\mathrm{ft}^{3} / \mathrm{s}\right)\end{array}$ & $\begin{array}{c}\mathrm{Q}_{7,2} \\
\left(\mathrm{ft}^{3} / \mathrm{s}\right)\end{array}$ & $\begin{array}{l}Q_{7,10} \\
\left(\mathrm{ft}^{3} / \mathrm{s}\right)\end{array}$ & $\begin{array}{c}\text { Accuracy } \\
\text { level }\end{array}$ \\
\hline LC4 & Isabelle Creek & $\begin{array}{l}\text { NE1/4NE1/2 sec. } 20, \text { T. } 26 \text { N., R. } 17 \text { W., } \\
\text { Pierce County, at sewage-treatment } \\
\text { plant, } 1.2 \text { mi east of Ellsworth, Wis. }\end{array}$ & 2.81 & $\begin{array}{l}\text { June } 26,1972 \\
\text { Aug. } 16,1972 \\
\text { June } 29,1973 \\
\text { Aug. } 7,1973 \\
\text { July } 30,1974 \\
\text { Aug. } 24,1976 \\
\text { Aug. } 9,1977\end{array}$ & $\begin{array}{l}1.38 \\
.22 \\
1.41 \\
1.06 \\
1.71 \\
.40 \\
1.05\end{array}$ & 5 & $\underline{5}$ & \\
\hline LC6 & $\begin{array}{l}\text { Rush River } \\
\text { tributary }\end{array}$ & $\begin{array}{l}\text { NW/ NWW/ sec. } 36, \text { T. } 29 \text { N., R. } 17 \text { W., } \\
\text { St. Croix County, at bridge on } \\
\text { country road, } 1.1 \text { mi southwest of } \\
\text { Baldwin, Wis. }\end{array}$ & 4.30 & $\begin{array}{l}\text { June } 27,1972 \\
\text { Aug. } 16,1972 \\
\text { June } 29,1973 \\
\text { Aug. } 8,1973 \\
\text { July } 30,1974 \\
\text { Aug. } 23,1976\end{array}$ & $\begin{array}{l}.46 \\
.17 \\
.51 \\
.58 \\
.60 \\
.25\end{array}$ & 5 & 5 & \\
\hline LC12 & Chippewa River & $\begin{array}{l}\text { SE1/4NE1// sec. 12, T. } 28 \text { N., R. } 9 \text { W., } \\
\text { Chippewa County, on right bank at } \\
\text { Chippewa Falls, } 1.0 \text { mi downstream } \\
\text { from Duncan Creek. }\end{array}$ & 5,670 & & & 1,440 & 794 & $\mathbf{a}$ \\
\hline LC19 & Chippewa River & $\begin{array}{l}\text { SW/ sec. } 21, \text { T. } 25 \text { N., R. } 13 \mathrm{~W} ., \\
\text { Pepin County, on left bank at } \\
\text { Durand, } 75 \mathrm{ft} \text { downstream from } \\
\text { U.S. Highway } 10 \text { bridge, } 9.5 \mathrm{mi} \\
\text { downstream from Red Cedar River. }\end{array}$ & 9,010 & & & 2,930 & 2,080 & $\mathbf{a}$ \\
\hline LC23 & Yellow River & $\begin{array}{l}\text { SWY/NWY/a sec. } 24, \text { T. } 31 \text { N., R. } 4 \text { W., } \\
\text { Taylor County, just upstream from } \\
\text { sewage lagoon, } 0.8 \text { mi southwest } \\
\text { of Gilman, Wis. }\end{array}$ & 193 & $\begin{array}{l}\text { June } 28,1972 \\
\text { Aug. } 15,1972 \\
\text { June } 26,1973 \\
\text { Aug. } 9,1973 \\
\text { July } 29,1974 \\
\text { Aug. } 25,1976\end{array}$ & $\begin{array}{c}7.33 \\
110 \\
23.6 \\
16.1 \\
6.05 \\
3.36\end{array}$ & 4.8 & 2.2 & c \\
\hline LC24 & Yellow River & $\begin{array}{l}\text { SE1/\%WW// sec. 31, T. } 29 \text { N., R. } 6 \text { W., } \\
\text { Chippewa County, } 0.1 \text { mi north of } \\
\text { State Highwey } 29,0.7 \text { mi west of } \\
\text { junction of State Highweys } 27 \text { and } \\
\text { 29, at Cadott, Wis. }\end{array}$ & 351 & $\begin{array}{l}\text { June } 27,1972 \\
\text { Aug. } 14,1972 \\
\text { Aug. } 8,1973 \\
\text { June } 26,1973 \\
\text { July } 30,1974 \\
\text { Aug. } 26,1976\end{array}$ & $\begin{array}{c}320.1 \\
121 \\
29.4 \\
56.7 \\
16.9 \\
6.90\end{array}$ & 11 & 5.2 & $\mathbf{a}$ \\
\hline LC28 & Duncan Creek & $\begin{array}{l}\text { SE } 1 / 4 \text { NWY/ sec. 1, T. } 31 \text { N., R. } 10 \text { W., } \\
\text { Chippewa County, at sewage-treetment } \\
\text { plant, at New Auburn, Wis. }\end{array}$ & .49 & July 31, 1974 & ${ }^{4} 0$ & 0 & 0 & c \\
\hline & Duncan Creek & $\begin{array}{l}\text { NE1/4NE1/ sec. 8, T. } 31 \text { N., R. } 9 \text { W., } \\
\text { Chippewa County, at bridge on } \\
\text { County Trunk AA, } 3.0 \text { mi } \\
\text { southeast of New Auburn, Wis. }\end{array}$ & 13.1 & $\begin{array}{l}\text { June } 28,1972 \\
\text { Aug. } 15,1972 \\
\text { June } 27,1973 \\
\text { Aug. } 9,1973 \\
\text { Aug. } 25,1976 \\
\text { Aug. } 25,1977\end{array}$ & $\begin{array}{l}3.61 \\
1.80 \\
1.66 \\
4.53 \\
.87 \\
.74\end{array}$ & .63 & .36 & c \\
\hline LC30 & Duncan Creek & $\begin{array}{l}\text { NE1/4NE1/4 sec. } 8, T .30 \text { N., R. } 9 \text { W., } \\
\text { Chippewe County, on left bank } \\
0.3 \text { mi below Bloomer dam, at } \\
\text { Bloomer, Wis. }\end{array}$ & 49.2 & Oct. 25,1972 & $3_{50.4}$ & 10 & 6.7 & $\mathbf{a}$ \\
\hline & Duncan Creek & $\begin{array}{l}\text { NWY/4SWY/4 sec. 9, T. } 30 \text { N., R. } 9 \text { W., } \\
\text { Chippewa County, at sewage-treatment } \\
\text { plant, at Bloomer, Wis. }\end{array}$ & 52.8 & & & & & \\
\hline LC38 & $\begin{array}{l}\text { North Fork } \\
\text { Eau Cleire } \\
\text { River tributary }\end{array}$ & $\begin{array}{l}\text { NWY NWY/4 sec. } 31, \text { T. } 29 \text { N., R. } 3 \text { W., } \\
\text { Clark County, at bridge on County } \\
\text { Trunk M, at Thorp, Wis. }\end{array}$ & 2.12 & $\begin{array}{l}\text { June } 28,1972 \\
\text { Aug. } 15,1972 \\
\text { Aug. } 8,1973 \\
\text { July } 30,1974\end{array}$ & $\begin{array}{l}4.48 \\
1.30 \\
.30 \text { est. }\end{array}$ & . & 0 & c \\
\hline LC40 & Wolf River & $\begin{array}{l}\text { SE1/\%NW\% sec. } 36, \text { T. } 29 \text { N., R. } 5 \text { W., } \\
\text { Chippewa County, at sewage-treatment } \\
\text { plant, at Stanley, Wis. }\end{array}$ & 27.6 & $\begin{array}{l}\text { June } 28,1972 \\
\text { Aug. } 15,1972 \\
\text { June } 26,1973 \\
\text { Aug. } 8,1973 \\
\text { July } 30,1974 \\
\text { Aug. } 25,1976\end{array}$ & $\begin{array}{l}1.96 \\
2.74 \\
4.33 \\
4.16 \\
4.19\end{array}$ & 6 & 6 & \\
\hline
\end{tabular}


Table 4. Lower Chippewa River basin station locations and low-flow characteristics-Continued.

\begin{tabular}{|c|c|c|c|c|c|c|}
\hline $\begin{array}{l}\text { Station }{ }^{1} \\
\text { number }\end{array}$ & $\underset{\text { name }}{\text { Stream }}$ & Station location & $\begin{array}{l}\text { Drainage } \\
\text { araa } \\
\left(\mathrm{mi}^{2}\right)\end{array}$ & Date & $\begin{array}{c}\text { Discharge } \\
\left(\mathrm{ft}^{3} / \mathrm{s}\right)\end{array}$ & $\begin{array}{ll}0_{7,2} & 0_{7,10} \\
\left(\mathrm{ft}^{3} / \mathrm{s}\right)\left(\mathrm{ft}^{3} / \mathrm{s}\right) & \text { Accuracy } \\
\text { level } & \end{array}$ \\
\hline
\end{tabular}

LC41 Black Creek tributary

Black Creek tributary

LC43 Hay Creek

Hay Creek tributary

LC44 Bridge Creek

LC47 Fall Creek

LC52 Red Cedar River

LC59 Red Cedar River

LC60, Brill River 61

LC65 Cranberry Creek

Cranberry Creek
NE $1 / 4$ NW $1 / 4$ sec. 2, T. 24 N., R. 5 W., Jackson County, at sewage lagoon, $0.5 \mathrm{mi}$ south of Fairchild, Wis.

NE $1 / 4$ NW $1 / 4$ sec. 2, T. 24 N., R. 5 W., Jackson County, at bridge on U.S. Highways 10 and 12, at Fairchild, Wis.

SEY/4SWT// sec. 29, T. 29 N., R. 5 W., Chippewa County, at culvert on country road, $1.1 \mathrm{mi}$ northeast of Boyd, Wis.

SE $1 / 4$ NE $/ 1$ sec. 31. T. 29 N., R. 5 W., Chippewa County, at sewagetreatment plant, at Boyd, Wis.

SE1/4SE1/4 sec. 32, T. 26 N., R. 6 W., Eau Claire County, at sewagetreatment plant, at Augusta, Wis.

NWY/4NE1/4 sec. 6, T. 26 N., R. 7 W., Eau Claire County, at sewagetreatmant plant, 0.3 mi north of U.S. Highway 12, at Fall Creek, Wis.

NWY $/ 4 W 1 / 4$ sec. 25, T. 37 N., R. 10 W. Washburn County, at culvert on County Trunk D, at Birchwood, Wis.

NW1/4NW1/4 sec. 26, T. 28 N., R. 13 W., 1,760

Dunn County, on right bank at Manomonie, $900 \mathrm{ft}$ downstream from powerplant of Northern States Power Company and $1,000 \mathrm{ft}$ downstream from Wilson Creek.

SE $1 / 2 S W \% / /$ sec. 12, T. 36 N., R. 11 W., Barron County, at bridge on County Trunk V, 0.5 mi north of Brill, Wis.

SW $/ 4 S E 1 / 4$ sec. 20, T. 34 N., R. 11 W., Barron County, at culvert on U.S. Highway 8, at Cameron, Wis.

SE1/4NE1/4 sec. 29, T. 34 N., R. 11 W., at sewage-treatment plant, at Cameron, Wis.

\subsection{1}

10.1

June 27, 1972

Aug. 14, 1972

June 25, 1973

Aug. 9, 1973

July 30,1974

Aug. 26, 1976

June 27, 1972

Aug, 15, 1972

June 26, 1973

Aug. 8, 1973

July 30,1974

Aug. 26, 1976

2.73

34.9

June 27, 1972

Aug. 14, 1972

June 25, 1973

Aug. 9, 1973

July 30,1974

Aug. 26, 1976

15.8

Aug. 14, 1972

June 25, 1973

Aug. 9, 1973

July 30,1974

Aug. 24, 1976

69.5 June 13,1972

Aug. 16, 1972

June 28, 1973

Aug. 8, 1973

Aug. 1, 1974

Aug. 26, 1976

Aug. 24, 1977
2.68
4.10
7.30
5.76
3.37
2.38
1.05
1.09
1.45
1.44
.62
.50

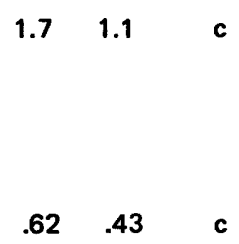

$\begin{array}{lll}4.2 & 2.6 \quad c\end{array}$

10.2

23.0

56.9

6.77

4.70

1.88

2.49

4.52

9.61

1.95

1.24

14.6

21.0

20.7

28.9

11.6

2.40

5.47

$\begin{array}{lll}1.1 & .68 \quad c\end{array}$

$\begin{array}{lll}3.2 & 1.5 \quad c\end{array}$
June 27, 1972

602

427

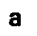

94.3 Oct. 25, 1972

June 28, 1973

Aug. 9, 1973

Aug. 1, 1974

Aug. 26, 1976

Aug. 23, 1977

Jan. 19. 1965

Oct. 26, 1972

June 27, 1973

Aug. 9, 1973

July 31,1974

Aug. 25, 1976

Aug. 23, 1977

5.03
52.0

54.7

52.5

30.7

24.9

26.9

2.91

5.23

4.67

3.91

2.26

1.97

1.36
$20 \quad 11 \quad c$

$\begin{array}{lll}1.2 \quad 5 & \mathrm{c}\end{array}$ 
Table 4. Lower Chippewa River basin station locations and low-flow characteristics-Continued.

\begin{tabular}{|c|c|c|c|c|c|c|c|c|}
\hline $\begin{array}{l}\text { Station } \\
\text { number }\end{array}$ & $n^{1} \underset{\text { name }}{\text { Stream }}$ & Station location & $\begin{array}{l}\text { Drainage } \\
\text { area } \\
\left(\mathrm{mi}^{2}\right)\end{array}$ & Date & $\begin{array}{l}\text { Discharge } \\
\left(\mathrm{ft}^{3} / \mathrm{s}\right)\end{array}$ & $\begin{array}{l}\mathrm{O}_{7,2} \mathrm{O}_{7} \\
\left(\mathrm{ft}^{3} / \mathrm{s}\right)(\mathrm{ft}\end{array}$ & $\begin{array}{l}7,10 \\
\left.t^{3} / s\right)\end{array}$ & $\underset{\text { level }}{\text { Accuracy }}$ \\
\hline LC66 & Yellow River & $\begin{array}{l}\text { NW1/4SE } 1 / 4 \text { sec. } 27, \text { T. } 34 \text { N., R. } 12 \text { W., } \\
\text { Barron County, just downstream from } \\
\text { dam and upstream from Quaderer } \\
\text { Creek, in Barron, Wis. }\end{array}$ & 146 & $\begin{array}{l}\text { Oct. } 25,1972 \\
\text { June } 27,1973 \\
\text { Aug. } 9,1973 \\
\text { July } 31,1974 \\
\text { Aug. } 25,1976 \\
\text { Aug. } 24,1977\end{array}$ & $\begin{array}{l}148 \\
117 \\
88.1 \\
52.6 \\
44.6 \\
42.2\end{array}$ & 29 & 14 & c \\
\hline LC67 & Chetek River & $\begin{array}{l}\text { NW } 1 / 4 \text { SW1/4 sec. } 31, \text { T. } 33 \text { N., R. } 10 \text { W., } \\
\text { Barron County, just downstream } \\
\text { from Tenmile Creek, } 1.4 \mathrm{mi} \\
\text { southwest of Chetek, Wis. }\end{array}$ & 194 & $\begin{array}{l}\text { Oct. } 25,1972 \\
\text { June } 27,1973 \\
\text { Aug. } 8,1973 \\
\text { July } 31,1974 \\
\text { Aug. } 26,1976 \\
\text { Aug. } 24,1977\end{array}$ & $\begin{array}{l}198 \\
143 \\
112 \\
43.2 \\
47.2 \\
42.5\end{array}$ & 28 & 11 & c \\
\hline \multirow[t]{3}{*}{ LC68 } & $\begin{array}{l}\text { Upper Pine } \\
\text { Creek }\end{array}$ & $\begin{array}{l}\text { SW1/4NW1/4 sec. } 11, T .32 \text { N., R. } 12 \text { W., } \\
\text { Barron County, et bridge on County } \\
\text { Trunk } U, 1.1 \text { mi north of Dallas, } \\
\text { Wis. }\end{array}$ & 8.85 & $\begin{array}{l}\text { Aug. } 15,1972 \\
\text { June } 27,1973 \\
\text { Aug. } 8,1973 \\
\text { July } 31,1974 \\
\text { Aug. } 25,1976 \\
\text { Aug. } 22,1977\end{array}$ & $\begin{array}{l}6.19 \\
6.13 \\
6.46 \\
4.75 \\
4.46 \\
4.21\end{array}$ & 3.8 & 2.9 & c \\
\hline & $\begin{array}{l}\text { East Branch } \\
\text { Upper Pine } \\
\text { Creek }\end{array}$ & $\begin{array}{l}\text { SW } 1 / 4 \text { SE } 1 / 4 \text { sec. } 11, T .32 \text { N., R. } 12 \text { W., } \\
\text { Barron County, at bridge on County } \\
\text { Trunk U, } 0.6 \text { mi north of Dallas, } \\
\text { Wis. }\end{array}$ & 9.60 & $\begin{array}{l}\text { Aug. } 8,1973 \\
\text { Aug. } 25,1976 \\
\text { Aug. 22, } 1977\end{array}$ & $\begin{array}{l}7.21 \\
3.18 \\
3.55\end{array}$ & 2.8 & 1.6 & c \\
\hline & $\begin{array}{l}\text { Upper Pine } \\
\text { Creek }\end{array}$ & $\begin{array}{l}\text { SW } 1 / 4 \text { SE } 1 / 4 \text { sec. } 14, T .32 \text { N., R. } 12 \text { W., } \\
\text { Barron County, at sewage-treatment } \\
\text { plant, at Dallas. Wis. }\end{array}$ & 19.7 & & & 6.2 & 3.6 & c \\
\hline LC69 & $\begin{array}{l}\text { Lower Pine } \\
\text { Creek tributary }\end{array}$ & $\begin{array}{l}\text { NE } 1 / 4 \text { NE } 1 / 4 \text { sec. } 6, \text { T. } 31 \text { N., R. } 12 \text { W., } \\
\text { Dunn County, at sewage-treatment } \\
\text { plant, at Ridgelend, Wis. }\end{array}$ & 7.94 & $\begin{array}{l}\text { June } 27,1972 \\
\text { Aug. } 15,1972 \\
\text { June } 27,1973 \\
\text { Aug. } 8,1973 \\
\text { July } 31,1974 \\
\text { Aug. } 24,1976 \\
\text { Aug. } 23,1977\end{array}$ & $\begin{array}{l}.86 \\
1.40 \\
1.38 \\
1.26 \\
.60 \\
.44 \\
.88\end{array}$ & .41 & .18 & c \\
\hline \multirow[t]{2}{*}{ LC71 } & Hay River & $\begin{array}{l}\text { SE } 1 / 4 \text { SE } 1 / 4 \text { sec. } 7, \text { T. } 35 \text { N., R. } 13 \text { W., } \\
\text { Barron County, at culvert on County } \\
\text { Trunk P, } 0.6 \text { mi south of Cumberland, } \\
\text { Wis. }\end{array}$ & 1.28 & $\begin{array}{lc}\text { Aug. } & 16,1972 \\
\text { June } & 28,1973 \\
\text { Aug. } & 9,1973 \\
\text { Aug. } & 1,1974\end{array}$ & $\begin{array}{l}10.8 \\
2.74 \\
2 \\
20\end{array}$ & $\underline{6}$ & $\underline{6}$ & \\
\hline & Hay River & $\begin{array}{l}\text { SW1/4NW1/4 sec. } 20, T .35 \mathrm{~N} ., \mathrm{R} .13 \mathrm{~W} ., \\
\text { Barron County, at culvert on country } \\
\text { road, } 2.1 \text { mi south of Cumberland, } \\
\text { Wis. }\end{array}$ & & $\begin{array}{lc}\text { June } & 28,1973 \\
\text { Aug. } & 9,1973 \\
\text { Aug. } & 1,1974\end{array}$ & $2_{0}^{9.14}$ & $\underline{6}$ & $\underline{6}$ & \\
\hline \multirow[t]{2}{*}{ LC72 } & Hay River & $\begin{array}{l}\text { SW1/4NE } 1 / 4 \text { sec. } 16, \text { T. } 32 \text { N., R. } 13 \text { W., } \\
\text { Barron County, at bridge on County } \\
\text { Trunk F, } 1.8 \text { mi north of Prairie } \\
\text { Ferm, Wis. }\end{array}$ & 80.5 & $\begin{array}{l}\text { Oct. } 26,1972 \\
\text { June } 27,1973 \\
\text { Aug. } 8,1973 \\
\text { July } 31,1974 \\
\text { Aug. } 24,1976\end{array}$ & $\begin{array}{l}53.1 \\
50.6 \\
47.1 \\
31.4 \\
29.7\end{array}$ & 22 & 14 & c \\
\hline & Hay River & $\begin{array}{l}\text { NE } 1 / 4 \text { NW1/4 sec. } 28, \text { T. } 32 \text { N., R. } 13 \text { W., } \\
\text { Barron County, } 0.3 \text { mi upstream from } \\
\text { Turtle Creek, at Prairie Farm, Wis. }\end{array}$ & 84.8 & & & & & \\
\hline LC74 & $\begin{array}{l}\text { Lightning } \\
\text { Creek }\end{array}$ & $\begin{array}{l}\text { NW1/ sec. 19, T. } 34 \text { N., R. } 13 \text { W., } \\
\text { Berron County, at bridge on County } \\
\text { Trunk P, in Almena, Wis. }\end{array}$ & 19.0 & $\begin{array}{l}\text { June } 28,1973 \\
\text { Aug. } 9,1973 \\
\text { July } 31,1974 \\
\text { Aug. } 25,1976\end{array}$ & $\begin{array}{l}3.50 \\
3.88 \\
1.02 \\
1.43\end{array}$ & .59 & .20 & c \\
\hline
\end{tabular}

NW $1 / 4$ SW $1 / 4$ sec. 19, T. 34 N., R. 13 W., Berron County, at sewage-treatment plant, at Almena, Wis.
19.4 
Table 4. Lower Chippewa River basin station locations and low-flow characteristics-Continued.

\begin{tabular}{|c|c|c|c|c|c|c|c|c|}
\hline $\begin{array}{l}\text { Station } \\
\text { number }\end{array}$ & ${ }^{1} \underset{\text { name }}{\text { Stream }}$ & Station location & $\begin{array}{l}\text { Drainage } \\
\text { area } \\
\left(\mathrm{mi}^{2}\right)\end{array}$ & Date & $\begin{array}{l}\text { Discharge } \\
\left(\mathrm{ft}^{3} / \mathrm{s}\right)\end{array}$ & $\begin{array}{l}Q_{7,2} Q_{7} \\
\left(\mathrm{ft}^{3} / \mathrm{s}\right)\left(\mathrm{ft}^{3}\right.\end{array}$ & 10 & $\underset{\text { level }}{\text { Accuracy }}$ \\
\hline \multirow[t]{2}{*}{ LC76 } & Turtle Creek & $\begin{array}{l}\text { NE } 1 / 4 \text { NW } 1 / 4 \text { sec. } 27, T .34 \text { N., R. } 14 \text { W., } \\
\text { Barron County, at culvert on U.S. } \\
\text { Highway } 8,2.7 \text { mi northeast of } \\
\text { Turtle Lake, Wis. }\end{array}$ & & $\begin{array}{l}\text { Aug. } 16,1972 \\
\text { June } 28,1973 \\
\text { Aug. } 9,1973 \\
\text { Aug. } 1,1974 \\
\text { Aug. } 25,1976 \\
\text { Aug. } 23,1977\end{array}$ & $\begin{array}{l}8.09 \\
5.96 \\
4.66 \\
1.92 \\
1.57 \\
1.96\end{array}$ & 1.1 & .42 & c \\
\hline & $\begin{array}{l}\text { Turtle Creek } \\
\text { tributary }\end{array}$ & $\begin{array}{l}\text { SW\% } 1 / \text { SE } 1 / 4 \text { sec. } 28, \text { T. } 34 \text { N., R. } 14 \text { W., } \\
\text { Barron County, at sewage-treatment } \\
\text { plant, } 1.9 \text { mi east of Turtle Lake, } \\
\text { Wis. }\end{array}$ & 2.37 & & & & & \\
\hline \multirow[t]{2}{*}{ LC79 } & Tiffany Creek & $\begin{array}{l}\text { NE } 1 / 4 N^{1} 1 / 4 \text { sec. } 35, T .30 \text { N., R. } 15 \text { W., } \\
\text { St. Croix County, at bridge on } \\
\text { country road, } 1.0 \text { mi southeast of } \\
\text { Glenwood City, Wis. }\end{array}$ & 14.0 & $\begin{array}{l}\text { June } 27,1972 \\
\text { Aug. } 16,1972 \\
\text { June } 28,1973 \\
\text { Aug. } 8,1973 \\
\text { July } 30,1974 \\
\text { Aug. } 24,1976\end{array}$ & $\begin{array}{l}4.53 \\
6.14 \\
6.29 \\
5.54 \\
4.14 \\
5.58\end{array}$ & 3.1 & 2.0 & c \\
\hline & Tiffany Creek & $\begin{array}{l}\text { SE } 1 / 4 \text { SW1/4 sec. } 25, \text { T. } 30 \text { N., R. } 15 \text { W., } \\
\text { St. Croix County, at sewage lagoons, } \\
1.3 \text { mi southeast of Glenwood, Wis. }\end{array}$ & 16.1 & & & & & \\
\hline \multirow[t]{2}{*}{ LC80 } & Tiffany Creek & $\begin{array}{l}\text { SE1/4SE1/4 sec. } 26, T .30 \text { N., R. } 14 \text { W., } \\
\text { Dunn County, at bridge on State } \\
\text { Highway } 79 \text {, at Boyceville, Wis. }\end{array}$ & 73.3 & $\begin{array}{l}\text { June } 27,1972 \\
\text { Oct. } 26,1972 \\
\text { June } 28,1973 \\
\text { Aug. } 8,1973 \\
\text { July } 30,1974 \\
\text { Aug. } 24,1976 \\
\text { Aug. } 23,1977\end{array}$ & $\begin{array}{l}19.5 \\
38.2 \\
29.5 \\
21.3 \\
18.6 \\
17.5 \\
19.1\end{array}$ & 13 & 8.2 & c \\
\hline & Tiffany Creek & $\begin{array}{l}\text { SW } 1 / 4 \text { NW } 1 / 4 \text { sec. } 30, T .30 \text { N., R. } 13 \text { W., } \\
\text { Dunn County, at sewage lagoon, } \\
1.2 \text { mi northeast of Boyceville, } \\
\text { Wis. }\end{array}$ & 77.3 & & & & & \\
\hline \multirow[t]{2}{*}{ LC82 } & Wilson Creek & $\begin{array}{l}\text { NW1/4SE } 1 / 4 \text { sec. } 35, \text { T. } 29 \text { N., R. } 14 \text { W., } \\
\text { Dunn County, at sewage-stabilization } \\
\text { pond, } 2.1 \text { mi east of Knapp, Wis. }\end{array}$ & 27.5 & & & & & \\
\hline & Wilson Creek & $\begin{array}{l}\text { NE } 1 / 4 N^{1} 1 / 4 \text { sec. } 2, T .28 \text { N., R. } 14 \text { W., } \\
\text { Dunn County, at bridge on U.S. } \\
\text { Highway } 12,2.3 \text { mi southeast of } \\
\text { Knepp, Wis. }\end{array}$ & 28.4 & $\begin{array}{l}\text { June } 27,1972 \\
\text { Oct. } 25,1972 \\
\text { June } 29,1973 \\
\text { Aug. } 7,1973 \\
\text { July } 30,1974 \\
\text { Aug. } 23,1976\end{array}$ & $\begin{array}{l}11.3 \\
14.7 \\
18.6 \\
18.6 \\
13.6 \\
12.8\end{array}$ & 9.2 & 5.9 & c \\
\hline LC86 & $\begin{array}{l}\text { Eau Galle } \\
\text { River tributary }\end{array}$ & $\begin{array}{l}\text { NE } 1 / 4 N W 1 / 4 \text { sec. } 2, \text { T. } 28 \text { N., R. } 16 \text { W., } \\
\text { St. Croix County, at sewage lagoon, } \\
0.8 \text { mi south of Woodville, Wis. }\end{array}$ & 11.7 & $\begin{array}{l}\text { Aug. } 15,1972 \\
\text { June } 29,1973 \\
\text { Aug. } 8,1973 \\
\text { July } 30,1974\end{array}$ & $\begin{array}{l}2.01 \\
2 \\
2 \\
2 \\
0\end{array}$ & $\mathbf{0}$ & 0 & c \\
\hline LC87 & Eau Galle River & $\begin{array}{l}\text { NE } 1 / 4 S^{1} 1 / 4 \text { sec. } 6, T .27 \text { N., R. } 15 \text { W., } \\
\text { Pierce County, on town road near } \\
\text { center of bridge at Spring Valley, } \\
0.1 \text { mi upstream from Mines Creek, } \\
0.5 \text { mi downstream from Lousy Creek. }\end{array}$ & 64.8 & & & 7.6 & 6.4 & $\mathbf{a}$ \\
\hline LC89 & Eau Galle River & $\begin{array}{l}\text { NW } 1 / 4 \text { SE } 1 / 4 \text { sec. } 35, \text { T. } 27 \text { N., R. } 15 \text { W., } \\
\text { Pierce County, on downstream right } \\
\text { side of highway bridge in Elmwood, } \\
2.0 \text { mi upstream from Cady Creek. }\end{array}$ & 91.9 & & & 15 & 11 & $\mathbf{a}$ \\
\hline LC9O & Eau Galle River & $\begin{array}{l}\text { SW1/4NW1/4 sec. } 31, \text { T. } 26 \text { N., R. } 13 \text { W., } \\
\text { Dunn County, at County Trunk D, } \\
\text { in Eau Galle, Wis. }\end{array}$ & 182 & $\begin{array}{l}\text { Oct. } 26,1972 \\
\text { Aug. } 24,1976 \\
\text { Aug. } 9,1977\end{array}$ & $\begin{array}{l}97.3 \\
70.4 \\
64.2\end{array}$ & 44 & 36 & c \\
\hline
\end{tabular}


Table 4. Lower Chippewa River basin station locations and low-flow characteristics-Continued.

\begin{tabular}{|c|c|c|c|c|c|c|c|c|}
\hline $\begin{array}{l}\text { Station } \\
\text { number }\end{array}$ & Stream & Station location & $\begin{array}{l}\text { Drainage } \\
\text { area } \\
\left(\mathrm{mi}^{2}\right)\end{array}$ & Date & $\begin{array}{c}\text { Discharge } \\
\left(\mathrm{ft}^{3} / \mathrm{s}\right)\end{array}$ & $\begin{array}{l}\mathrm{Q}_{7,2} \mathrm{Q}_{7} \\
\left(\mathrm{ft}^{3} / \mathrm{s}\right)\left(\mathrm{ft}^{3}\right.\end{array}$ & & $\begin{array}{c}\text { Accuracy } \\
\text { level }\end{array}$ \\
\hline \multirow[t]{2}{*}{ LC92 } & Cady Creek & $\begin{array}{l}\text { NE1/4NE1/4 sec. 9, T. } 28 \text { N., R. } 15 \text { W., } \\
\text { St. Croix County, at bridga on } \\
\text { State Highway } 128,2.0 \text { mi southwest } \\
\text { of Wilson, Wis. }\end{array}$ & 1.68 & $\begin{array}{l}\text { July } 30 ; 1974 \\
\text { Aug. } 9,1977\end{array}$ & $\begin{array}{l}2_{0}^{0} \\
2_{0}^{2}\end{array}$ & 0 & 0 & c \\
\hline & Cady Creek & $\begin{array}{l}\text { SE1/4NE1/4 sec. } 22, T .28 \text { N., R. } 15 \text { W., } \\
\text { St. Croix County, at bridge on country } \\
\text { road, } 4.5 \text { mi northeast of Spring } \\
\text { Valley, Wis. }\end{array}$ & 6.24 & $\begin{array}{l}\text { July } 30,1974 \\
\text { Aug. } 23,1976 \\
\text { Aug. } 9,1977\end{array}$ & $\begin{array}{l}{ }_{2}^{0} \\
2_{0}^{0}\end{array}$ & 0 & 0 & c \\
\hline LC93 & $\begin{array}{l}\text { Arkansaw } \\
\text { Creek }\end{array}$ & $\begin{array}{l}\text { SW1/4NWY// sec. } 24, \text { T. } 25 \text { N., R. } 14 \text { W., } \\
\text { Pepin County, at bridge on County } \\
\text { Trunk D and O, at Arkansaw, Wis. }\end{array}$ & 22.2 & $\begin{array}{l}\text { June } 26,1972 \\
\text { June } 29,1973 \\
\text { Aug. } 7,1973 \\
\text { July } 30,1974 \\
\text { Aug. } 23,1976\end{array}$ & $\begin{array}{l}4.85 \\
9.45 \\
9.30 \\
6.44 \\
5.80\end{array}$ & 3.8 & 3.2 & c \\
\hline LC94 & Plum Creek & $\begin{array}{l}\text { SEYNWWY// sec. 22, T. } 25 \text { N., R. } 15 \text { W., } \\
\text { Pierce County, at bridge on U.S. } \\
\text { Highway } 10 \text {, in Plum City, Wis. }\end{array}$ & 31.5 & & & 2.8 & 2.0 & b \\
\hline LC1A & Bear Creek & $\begin{array}{l}\text { NWY NE } 1 / 4 \text { sec. } 18, \text { T. } 36 \text { N., R. } 11 \text { W., } \\
\text { Barron County, ou tlet Bear Lake, } \\
\text { at Haugen, Wis. }\end{array}$ & 36.8 & $\begin{array}{l}\text { July } 14,1972 \\
\text { Aug. } 9,1972 \\
\text { Oct. } 24,1972 \\
\text { Aug. } 26,1976 \\
\text { Aug. } 23,1977\end{array}$ & $\begin{array}{r}14.4 \\
20.5 \\
21.0 \\
4.34 \\
7.57\end{array}$ & 5.6 & 3.0 & c \\
\hline
\end{tabular}

'Based on 1971 report by Wisconsin Department of Natural Resources (DNR). Site lettered "A" was not included in 1971 DNR report.

${ }^{2}$ Streambed is dry.

3 Additional discharge measurements are available.

${ }^{4}$ Negligible discharge--water ponded, unable to measure velocity.

${ }^{5}$ No estimate possible--discharge is primarily effluent.

${ }^{6}$ No estimate possible due to regulation upstream. 
Table 5. Upper Chippewa River basin station locations and low-flow characteristics.

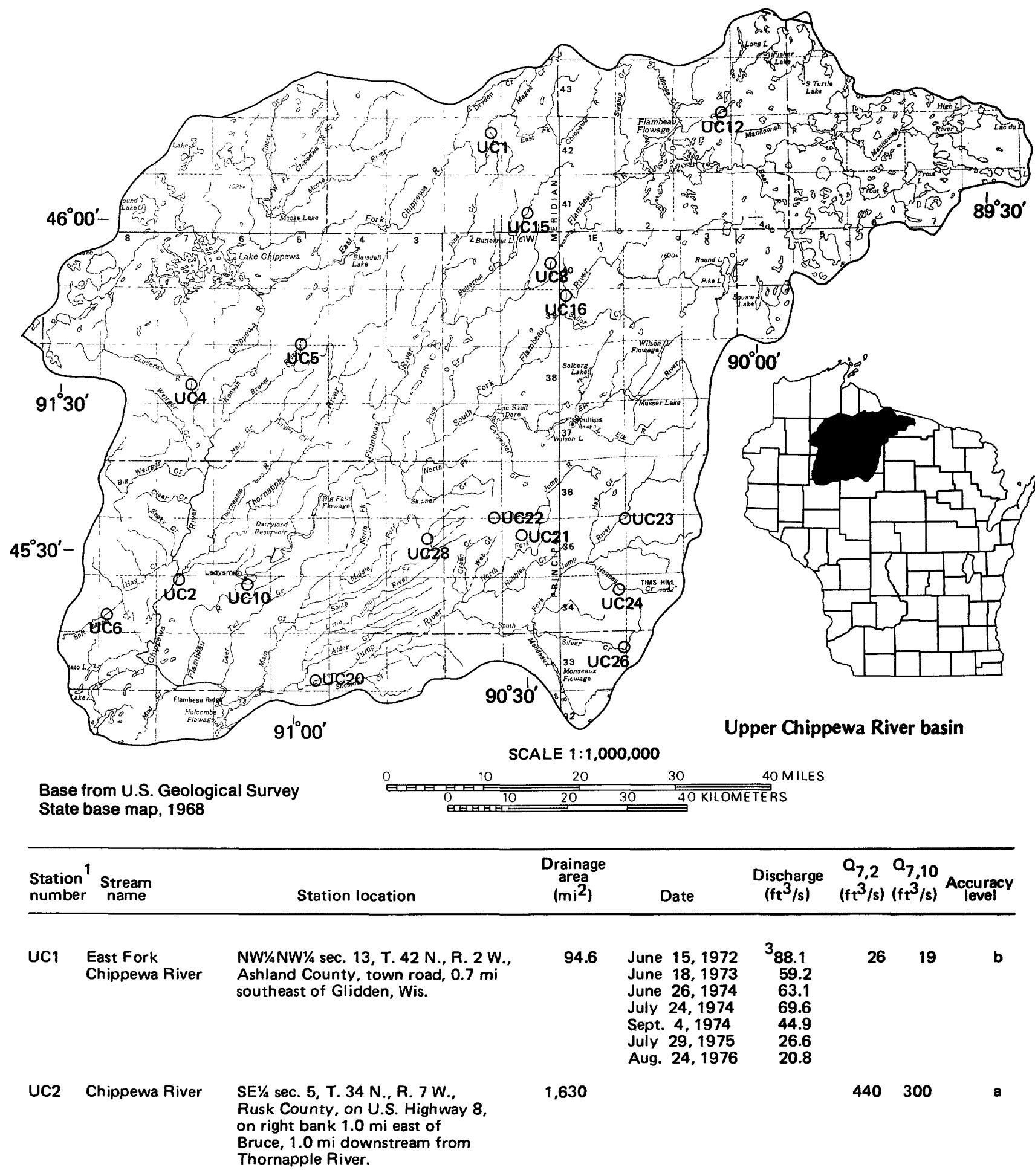


Table 5. Upper Chippewa River basin station locations and low-flow characteristics-Continued.

\begin{tabular}{|c|c|c|c|c|c|c|c|c|}
\hline $\begin{array}{l}\text { Station } \\
\text { number }\end{array}$ & Stream & Station location & $\begin{array}{l}\text { Drainage } \\
\text { area } \\
\left(\mathrm{mi}^{2}\right)\end{array}$ & Date & $\begin{array}{c}\text { Discharge } \\
\left(\mathrm{ft}^{3} / \mathrm{s}\right)\end{array}$ & $\begin{array}{l}\mathrm{O}_{7,2} \mathrm{O}_{7} \\
\left(\mathrm{ft}^{3} / \mathrm{s}\right)\left(\mathrm{ft}^{3}\right.\end{array}$ & $\begin{array}{l}7,10 \\
3 / 5)\end{array}$ & $\begin{array}{l}\text { Accuracy } \\
\text { level }\end{array}$ \\
\hline \multirow[t]{2}{*}{ UC4 } & Couderay River & $\begin{array}{l}\text { SE } 1 / 4 \text { SW } 1 / 4 \text { sec. } 17, \text { T. } 38 \text { N., R. } 7 \text { W., } \\
\text { Sawyer County, at bridge on } \\
\text { country road, } 2.2 \text { mi west of } \\
\text { Radisson, Wis. }\end{array}$ & 187 & $\begin{array}{l}\text { June } 19,1973 \\
\text { Oct. } 17,1973 \\
\text { July } 24,1974 \\
\text { Sept. } 5,1974 \\
\text { July } 30,1975 \\
\text { Aug. } 25,1976\end{array}$ & $\begin{array}{l}116 \\
255 \\
89.0 \\
70.2 \\
82.1 \\
37.6\end{array}$ & 54 & 31 & c \\
\hline & Couderay River & $\begin{array}{l}\text { NW } 1 / 4 \text { SE } 1 / 4 \text { sec. } 22, \text { T. } 38 \text { N., R. } 7 \text { W., } \\
\text { Sawyer County, at dam, } 0.6 \text { mi south } \\
\text { of Radisson, Wis. }\end{array}$ & 206 & & & & & \\
\hline UC5 & Brunet River & $\begin{array}{l}\text { SE } 1 / 4 N E 1 / 4 \text { sec. } 9, \text { T. } 38 \text { N., R. } 5 \text { W., } \\
\text { Sawyer County, at bridge on County } \\
\text { Trunk W, } 2.1 \text { mi southeast of } \\
\text { Winter, Wis. }\end{array}$ & 58.7 & $\begin{array}{l}\text { Aug. } 23,1967 \\
\text { June } 13,1972 \\
\text { June } 19,1973 \\
\text { Oct. } 17,1973 \\
\text { July } 24,1974 \\
\text { Sept. } 5,1974 \\
\text { July } 30,1975 \\
\text { Aug. } 25,1976\end{array}$ & $\begin{array}{l}17.4 \\
17.0 \\
35.9 \\
20.3 \\
18.8 \\
18.9 \\
17.4 \\
8.84\end{array}$ & 12 & 7.8 & c \\
\hline UC6 & $\begin{array}{l}\text { Soft Maple } \\
\text { Creek }\end{array}$ & $\begin{array}{l}\text { SE } 1 / 4 S E 1 / / \text { sec. } 19, \text { T. } 34 \text { N., R. } 8 \text { W., } \\
\text { Rusk County, at bridge on County } \\
\text { Trunk F, } 1.2 \text { mi southeast of } \\
\text { Weyerhauser, Wis. }\end{array}$ & 18.4 & $\begin{array}{l}\text { June } 13,1972 \\
\text { June } 19,1973 \\
\text { Aug. } 8,1973 \\
\text { July } 24,1974 \\
\text { Sept. } 5,1974 \\
\text { July } 30,1975 \\
\text { Aug. } 25,1976\end{array}$ & $\begin{array}{l}5.03 \\
6.72 \\
7.96 \\
1.94 \\
2.21 \\
2.00 \\
1.99\end{array}$ & 1.8 & 1.3 & c \\
\hline UC8 & Flambeau River & $\begin{array}{l}\text { NW } 1 / 4 \text { SW } 1 / 4 \text { sec. } 24, \text { T. } 40 \text { N., R. } 1 \text { W., } \\
\text { Price County, at sewage-treatment } \\
\text { plant, at Park Falls, Wis. }\end{array}$ & 769 & & & 500 & 260 & $\mathbf{a}$ \\
\hline UC10 & Flambeau River & $\begin{array}{l}\text { S1/a sec. } 35, \text { T. } 36 \text { N., R. } 5 \text { W., } \\
\text { Rusk County, at Big Falls power- } \\
\text { plant of Lake Superior District } \\
\text { Power Company, } 2.3 \text { mi upstream } \\
\text { from Josie Creek, } 4.0 \text { mi upstream } \\
\text { from highway bridge, } 8.5 \text { mi northeast } \\
\text { of Ladysmith, Wis. }\end{array}$ & 1,820 & & & 710 & 435 & $\mathbf{a}$ \\
\hline UC12 & $\begin{array}{l}\text { Little Turtle } \\
\text { River }\end{array}$ & $\begin{array}{l}\text { SW1/4NE1/4 sec. } 36, \text { T. } 43 \text { N., R. } 3 \text { E., } \\
\text { Iron County, at bridge on U.S. } \\
\text { Highway } 51 \text {, at Mercer, Wis. }\end{array}$ & 9.77 & $\begin{array}{l}\text { June } 15,1972 \\
\text { June } 18,1973 \\
\text { Aug. } 7,1973 \\
\text { July } 23,1974 \\
\text { Sept. } 4,1974 \\
\text { July } 29,1975 \\
\text { Aug. } 24,1976\end{array}$ & $\begin{array}{c}7.38 \\
12.9 \\
6.10 \\
5.55 \\
4.95 \\
4.68 \\
4.08\end{array}$ & 4.1 & 3.2 & c \\
\hline UC15 & Butternut Creek & $\begin{array}{l}\text { NW1/4NE } 1 / 4 \text { sec. } 28, \text { T. } 41 \text { N., R. } 1 \text { W., } \\
\text { Ashland County, at sewage- } \\
\text { treatment plant ou tlet, in } \\
\text { Butternut, Wis. }\end{array}$ & 25.0 & $\begin{array}{l}\text { June } 15,1972 \\
\text { June } 18,1973 \\
\text { Oct. } 17,1973 \\
\text { July } 23,1974 \\
\text { Sept. } 4,1974 \\
\text { July } 29,1975 \\
\text { Aug. } 24,1976\end{array}$ & $\begin{array}{l}44.1 \\
16.2 \\
31.5 \\
20.0 \\
10.6 \\
6.08 \\
3.84\end{array}$ & 5.3 & 3.4 & c \\
\hline UC16 & $\begin{array}{l}\text { South Fork } \\
\text { Flambeau River }\end{array}$ & $\begin{array}{l}\text { NE } 1 / 4 N W 1 / / \text { sec. } 7 . \text { T. } 34 \text { N., R. } 1 \text { E., } \\
\text { Price County, at bridge on Center } \\
\text { Street, at Fifield. Wis. }\end{array}$ & 262 & $\begin{array}{l}\text { July } 29,1975 \\
\text { Aug. } 2,1976 \\
\text { Aug. } 24,1976 \\
\text { Aug. } 23,1977\end{array}$ & $\begin{array}{l}56.4 \\
35.1 \\
44.0 \\
76\end{array}$ & 52 & 36 & c \\
\hline UC20 & Jump River & $\begin{array}{l}\text { SW } 1 / 4 S E 1 / 4 \text { sec. } 26, \text { T. } 33 \text { N., R. } 5 \text { W., } \\
\text { Rusk County, on right bank, just } \\
\text { downstream from County Trunk C } \\
\text { bridge in Sheldon, } 1,500 \mathrm{ft} \text { upstream } \\
\text { from Shoulder Creek, } 11 \text { mi upstream } \\
\text { from mouth. }\end{array}$ & 574 & & & 35 & 20 & $\mathbf{a}$ \\
\hline UC21 & $\begin{array}{l}\text { North Fork } \\
\text { Jump River } \\
\text { tributary }\end{array}$ & $\begin{array}{l}\text { SW } 1 / 4 \text { SW } 1 / 4 \text { sec. } 16, T .35 \text { N., R. } 1 \text { W., } \\
\text { Price County, at bridge on country } \\
\text { road, } 2.0 \text { mi southeast of Catawba, } \\
\text { Wis. }\end{array}$ & 3.61 & $\begin{array}{l}\text { July } 28,1975 \\
\text { Aug. } 2,1976 \\
\text { Aug. } 23,1977\end{array}$ & $\begin{array}{l}{ }^{4}{ }_{0}^{0} \\
.05\end{array}$ & 0 & 0 & c \\
\hline
\end{tabular}


Table 5. Upper Chippewa River basin station locations and low-flow characteristics-Continued.

\begin{tabular}{|c|c|c|c|c|c|c|c|c|}
\hline $\begin{array}{l}\text { Station } \\
\text { number }\end{array}$ & ${ }_{\text {name }}^{1}$ & Station location & $\begin{array}{l}\text { Drainage } \\
\text { area } \\
\left(\mathrm{mi}^{2}\right)\end{array}$ & Date & $\begin{array}{c}\text { Discharge } \\
\left(\mathrm{ft}^{3} / \mathrm{s}\right)\end{array}$ & $\begin{array}{l}\mathrm{a}_{7,2} \mathrm{o}_{7,1} \\
\left(\mathrm{ft}^{3} / \mathrm{s}\right)\left(\mathrm{ft}^{3}\right)\end{array}$ & & $\underset{\text { level }}{\text { Accuracy }}$ \\
\hline UC22 & $\begin{array}{l}\text { Web Creek } \\
\text { tributary }\end{array}$ & $\begin{array}{l}\text { SW } 1 / 4 \text { NE } 1 / 4 \text { sec. } 23, T .35 \text { N., R. } 2 \text { W., } \\
\text { Price County, at bridge on Web } \\
\text { Creek Road, } 2.1 \text { mi south of Kennan, } \\
\text { Wis. }\end{array}$ & 5.65 & $\begin{array}{l}\text { July } 28,1975 \\
\text { Aug. } 2,1976 \\
\text { Aug. } 23,1977\end{array}$ & $\begin{array}{l}{ }^{2} 0 \\
0 \\
.10\end{array}$ & 0 & 0 & c \\
\hline UC23 & $\begin{array}{l}\text { South Fork } \\
\text { Jump River }\end{array}$ & $\begin{array}{l}\text { SE1/4NE1/4 sec. } 1, \text { T. } 35 \text { N., R. } 1 \text { E., } \\
\text { Price County, } 0.2 \text { mi west of } \\
\text { State Highway } 13 \text {, at Prentice, Wis. }\end{array}$ & 59.7 & $\begin{array}{l}\text { June } 14,1972 \\
\text { June } 19,1973 \\
\text { Oct. } 17,1973 \\
\text { July } 23,1974 \\
\text { Sept. } 3,1974 \\
\text { July } 28,1975 \\
\text { Aug. } 23,1976\end{array}$ & $\begin{array}{c}12.8 \\
31.6 \\
25.3 \\
.59 \\
2.75 \\
5.14 \\
.60\end{array}$ & 1.3 & .58 & c \\
\hline UC24 & Holmes Creek & $\begin{array}{l}\text { NW1/4SW } 1 / 4 \text { sec. } 12, \text { T. } 34 \text { N., R. } 1 \text { E., } \\
\text { Price County, at bridge on State } \\
\text { Highway } 86,0.6 \text { mi west of } \\
\text { Ogema, Wis. }\end{array}$ & 10.2 & $\begin{array}{l}\text { June } 14,1972 \\
\text { June } 19,1973 \\
\text { Oct. } 16,1973 \\
\text { July } 23,1974 \\
\text { Sept. } 4,1974 \\
\text { July } 28,1975 \\
\text { Aug. } 23,1976\end{array}$ & $\begin{array}{l}.44 \\
4.63 \\
2.35 \\
.27 \\
.13 \\
.27 \\
.05\end{array}$ & .09 & .03 & c \\
\hline UC26 & Silver Creek & $\begin{array}{l}\text { SW1/4SEY/4 sec. } 12, \text { T. } 33 \text { N., R. } 1 \text { E., } \\
\text { Taylor County, just downst ream } \\
\text { from State Highway } 13 \text {, at } \\
\text { Westboro, Wis. }\end{array}$ & 35.8 & $\begin{array}{l}\text { Aug. } 23,1967 \\
\text { June } 14,1972 \\
\text { June } 19,1973 \\
\text { Oct. } 16,1973 \\
\text { July } 23,1974 \\
\text { Sept. } 4,1974 \\
\text { July } 28,1975 \\
\text { Aug. } 23,1976\end{array}$ & $\begin{array}{c}1.17 \\
3.14 \\
35.6 \\
13.2 \\
2.55 \\
2.35 \\
.95 \\
.88\end{array}$ & 1.3 & .58 & c \\
\hline UC28 & $\begin{array}{l}\text { South Fork } \\
\text { Main Creek }\end{array}$ & $\begin{array}{l}\text { SW1/4SE } 1 / 4 \text { sec. } 14, \text { T. } 35 \mathrm{~N} ., \text { R. } 3 \mathrm{~W} . \text {., } \\
\text { Rusk County, at sewage-treatment } \\
\text { plant, at Hawkins, Wis. }\end{array}$ & 17.5 & $\begin{array}{l}\text { June } 14,1972 \\
\text { June } 19,1973 \\
\text { Aug. } 8,1973 \\
\text { July } 22,1974 \\
\text { Sept. } 3,1974 \\
\text { July } 28,1975 \\
\text { Aug. } 23,1976\end{array}$ & $\begin{array}{l}29.0 \\
4.80 \\
2.11 \\
1.14 \\
.70 \\
.50 \\
.16\end{array}$ & .33 & .11 & c \\
\hline
\end{tabular}

\footnotetext{
1 Based on 1970 report by Wisconsin Department of Natural Resources.

2 Streambed is dry.

${ }^{3}$ Additionel discharge measurements ere available.

${ }^{4}$ Negligible discharge--water ponded, unable to measure velocity.
} 
Table 6. Des Plaines-Pike River basin station locations and low-flow characteristics.
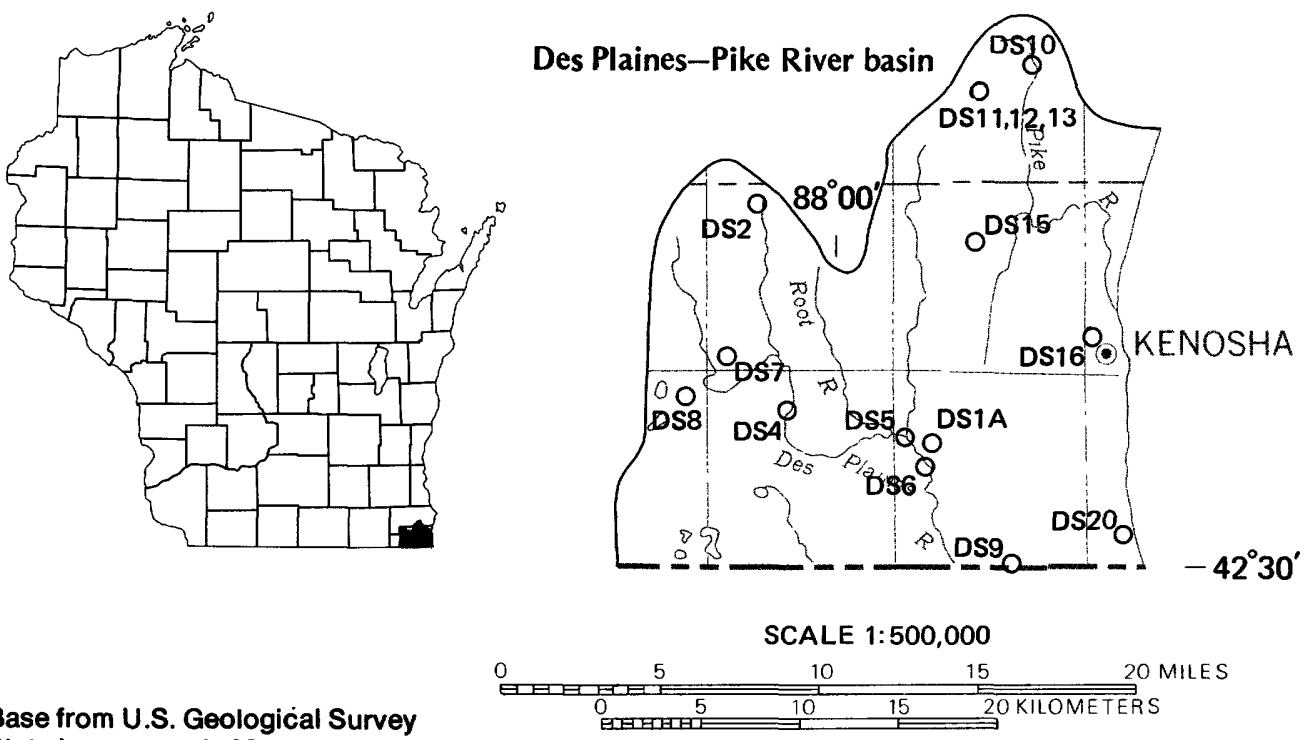

Base from U.S. Geological Survey State base map, 1968

\begin{tabular}{|c|c|c|c|c|c|c|c|c|}
\hline $\begin{array}{l}\text { Station } \\
\text { number }\end{array}$ & 1 Stream & Station location & $\begin{array}{l}\text { Drainage } \\
\text { area } \\
\left(\mathrm{mi}^{2}\right)\end{array}$ & Date & $\begin{array}{c}\text { Discharge } \\
\left(\mathrm{ft}^{3} / \mathrm{s}\right)\end{array}$ & $\begin{array}{l}\mathrm{Q}_{7,2} \mathrm{O}_{7} \\
\left(\mathrm{ft}^{3} / \mathrm{s}\right)\left(\mathrm{ft}^{3}\right.\end{array}$ & & Accuracy \\
\hline DS2 & Des Plaines River & $\begin{array}{l}\text { SE } 1 / 4 S^{1} 1 / 4 \text { sec. } 32, T .3 \text { N., R. } 21 \text { E., } \\
\text { Racine County, at County Trunk } \\
\text { KR, } 1.3 \text { mi southeast of Union } \\
\text { Grove, Wis. }\end{array}$ & 2.03 & $\begin{array}{l}\text { Sept. } 18,1974 \\
\text { Sept. 18, } 1975 \\
\text { Oct. } 20,1977\end{array}$ & $\begin{array}{l}{ }_{2}^{2} \\
2_{0}^{0}\end{array}$ & 0 & 0 & c \\
\hline \multirow[t]{2}{*}{ DS4 } & Des Plaines River & $\begin{array}{l}\text { SW } / 4 \text { SE } 1 / 4 \text { sec. } 4, T .1 \text { N., R. } 21 \text { E., } \\
\text { Kenosha County, at bridge on } \\
\text { State Highway } 50,1.6 \text { mi north- } \\
\text { east of Bristol, Wis. }\end{array}$ & 51.3 & $\begin{array}{l}\text { July } 18,1973 \\
\text { Aug. } 8,1973 \\
\text { Sept. } 18,1974 \\
\text { Sept. } 19,1975 \\
\text { Sept. } 2,1976\end{array}$ & $\begin{array}{c}3.78 \\
.97 \\
.42 \\
1.05 \\
.34\end{array}$ & .16 & $<.01$ & c \\
\hline & $\begin{array}{l}\text { Des Plaines } \\
\text { River tributary }\end{array}$ & $\begin{array}{l}\text { NE } 1 / 4 \text { SW } 1 / 4 \text { sec. } 8, T \text {. } 1 \text { N., R. } 21 \text { E., } \\
\text { Kenosha County, at sewage-treatment } \\
\text { plant, at Bristol, Wis. }\end{array}$ & .27 & & & & & \\
\hline DS5 & $\begin{array}{l}\text { Des Plaines } \\
\text { River }\end{array}$ & $\begin{array}{l}\text { SW } 1 / 4 \text { SW } 1 / 4 \text { sec. } 7, \text { T. } 1 \text { N., R. } 22 \text { E., } \\
\text { Kenosha County, at bridge on U.S. } \\
\text { Highway } 41 \text { and Interstate Highway } \\
94,1.0 \text { mi west of Pleasant } \\
\text { Prairie, Wis. }\end{array}$ & 71.5 & $\begin{array}{l}\text { July } 17,1973 \\
\text { Aug. } 8,1973 \\
\text { Sept. } 19,1974 \\
\text { Sept. } 19,1975 \\
\text { Oct. } 20,1977\end{array}$ & $\begin{array}{l}4.58 \\
.85 \\
.99 \\
2.12 \\
4.01\end{array}$ & .22 & $<.01$ & c \\
\hline \multirow[t]{2}{*}{ DS6 } & $\begin{array}{l}\text { Des Plaines } \\
\text { River }\end{array}$ & $\begin{array}{l}\text { SE } 1 / 4 \text { NW } 1 / 4 \text { sec. } 18, T .1 \text { N., R. } 22 \text { E., } \\
\text { Kenosha County, at bridge on } \\
\text { County Trunk C, } 0.6 \text { mi southwest } \\
\text { of Pleasant Prairie, Wis. }\end{array}$ & 95.6 & $\begin{array}{l}\text { July } 17,1973 \\
\text { Aug. } 8,1973 \\
\text { Sept. 19, } 1974 \\
\text { Sept. 19, } 1975 \\
\text { Oct. } 20,1977\end{array}$ & $\begin{array}{l}5.25 \\
.81 \\
1.07 \\
1.99 \\
6.16\end{array}$ & .23 & $<.01$ & c \\
\hline & $\begin{array}{l}\text { Des Plaines } \\
\text { River tributary }\end{array}$ & $\begin{array}{l}\text { NW1/4NW1/4 sec. 17, T. } 1 \text { N., R. } 22 \text { E., } \\
\text { Kenosha County, at sewage-treatment } \\
\text { plant, } 0.1 \text { mi southwest of County } \\
\text { Trunk C, at Pleasant Prairie, Wis. }\end{array}$ & .76 & & & & & \\
\hline \multirow[t]{2}{*}{ DS7 } & Brighton Creek & $\begin{array}{l}\text { NW1/4NW1/4 sec. } 6, \text { T. } 1 \text { N., R. } 21 \text { E., } \\
\text { Kenosha County, at bridge on } \\
\text { County Trunk K, } 1.8 \text { mi east of } \\
\text { Paddock Lake, Wis. }\end{array}$ & 16.6 & $\begin{array}{l}\text { July } 18,1973 \\
\text { Aug. } 9,1973 \\
\text { Sept. 18, } 1974 \\
\text { Sept. 17, } 1975\end{array}$ & $\begin{array}{l}1.84 \\
4_{0}^{.16} \\
{ }_{0}^{0}\end{array}$ & 0 & 0 & c \\
\hline & $\begin{array}{l}\text { Brighton Creek } \\
\text { tributary }\end{array}$ & $\begin{array}{l}\text { NE } 1 / 4 N^{1} 1 / 4 \text { sec. } 2, T .1 \text { N., R. } 20 \text { E., } \\
\text { Kenosha County, at sewage-disposal } \\
\text { plant at northeast edge of Paddock } \\
\text { Lake, Wis. }\end{array}$ & .99 & & & & & \\
\hline
\end{tabular}


Table 6. Des Plaines-Pike River basin station locations and low-flow characteristics-Continued.

\begin{tabular}{|c|c|c|c|c|c|c|c|c|}
\hline $\begin{array}{l}\text { Station } \\
\text { number }\end{array}$ & Stream & Station location & $\begin{array}{l}\text { Drainage } \\
\text { area } \\
\left(\mathrm{mi}^{2}\right)\end{array}$ & Date & $\begin{array}{c}\text { Discharge } \\
\left(\mathrm{ft}^{3} / \mathrm{s}\right)\end{array}$ & $\begin{array}{l}\mathrm{O}_{7,2} \mathrm{O}_{7} \\
\left(\mathrm{ft}^{3} / \mathrm{s}\right)\left(\mathrm{ft}^{3}\right.\end{array}$ & $3 / \mathrm{s}) \mathrm{Ac}$ & $\begin{array}{c}\text { Accuracy } \\
\text { level }\end{array}$ \\
\hline DS8 & Salem Branch & $\begin{array}{l}\text { SE1/aNE1/a sec. 11, T. } 1 \text { N., R. } 20 \text { E., } \\
\text { Kenosha County, just downstream } \\
\text { from confluence of tributaries from } \\
\text { Paddock Lake and Hooker Lake, } \\
1.2 \text { mi northeast of Salem, Wis. }\end{array}$ & 3.77 & $\begin{array}{l}\text { Sept. 19, } 1974 \\
\text { Sept. 17, } 1975 \\
\text { Sept. 6, } 1978\end{array}$ & $\begin{array}{l}{ }^{4} 0 \\
4_{0}^{0} \\
.94\end{array}$ & 0 & 0 & c \\
\hline \multirow[t]{2}{*}{ DS9 } & $\begin{array}{l}\text { Pleasant Homes } \\
\text { Drainage Course } \\
\text { tributary }\end{array}$ & $\begin{array}{l}\text { SW } 1 / 4 \text { SW } 1 / 4 \text { sec. } 36, \text { T. } 1 \text { N., R. } 22 \text { E., } \\
\text { Kenosha County, near Wis.-III. } \\
\text { border off County Trunk EZ, } 2.5 \text { mi } \\
\text { southwest of Carol Beach, Wis. }\end{array}$ & .46 & & & & & \\
\hline & $\begin{array}{l}\text { Pleasant Homes } \\
\text { Drainage Course }\end{array}$ & $\begin{array}{l}\text { SW1/4SE } 1 / 4 \text { sec. } 36, \text { T. } 1 \text { N., R. } 22 \text { E., } \\
\text { Kenosha County, at culvert on } \\
\text { State Line Road at Wis.-III. border, } \\
2.5 \text { mi southwest of Carol Beach, Wis. }\end{array}$ & .89 & $\begin{array}{l}\text { Sept. } 2,1972 \\
\text { July } 18,1973 \\
\text { Aug. } 8,1973 \\
\text { Sept. } 19,1974 \\
\text { Sept. } 19,1975\end{array}$ & $\begin{array}{l}.38 \\
.03 \\
<.05 \text { est. } \\
.01 \text { est. } \\
.02 \text { est. }\end{array}$ & $<.01$ & $<.01$ & c \\
\hline DS10 & Pike River & $\begin{array}{l}\text { SW1/4NW1/4 sec. } 14, \text { T. } 3 \text { N., R. } 22 \text { E., } \\
\text { Racine County, at culvert on State } \\
\text { Highway } 20,1.5 \text { mi west of Racine, } \\
\text { Wis. }\end{array}$ & 4.45 & $\begin{array}{l}\text { Sept. } 2,1972 \\
\text { July } 17,1973 \\
\text { Aug. } 8,1973 \\
\text { Oct. } 26,1973 \\
\text { Sept. 19, 1974 } \\
\text { Sept. 19, } 1975\end{array}$ & $\begin{array}{l}2.18 \\
.15 \\
.08 \\
.15 \\
.03 \\
.09\end{array}$ & .01 & $<.01$ & c \\
\hline DS11 & $\begin{array}{l}\text { Pike River } \\
\text { tributary }\end{array}$ & $\begin{array}{l}\text { SW } 1 / 4 \text { NE } 1 / 4 \text { sec. } 21, \text { T. } 3 \text { N., R. } 22 \text { E., } \\
\text { Racine County, at culvert on } \\
\text { Fancher Road, } 0.3 \text { mi north of } \\
\text { Sturtevant, Wis. }\end{array}$ & .66 & $\begin{array}{l}\text { Sept. } 2,1972 \\
\text { July } 17,1973 \\
\text { Aug. } 8,1973 \\
\text { Oct. } 26,1973 \\
\text { Sept. 19, 1974 } \\
\text { Sept. 18, 1975 }\end{array}$ & $\begin{array}{l}2.34 \\
20 \\
20 \\
20 \\
20 \\
20\end{array}$ & 0 & 0 & $c$ \\
\hline${ }_{13}^{\text {DS12, }}$ & $\begin{array}{l}\text { Pike River } \\
\text { tributary }\end{array}$ & $\begin{array}{l}\text { NE } 1 / 4 \text { SW } 1 / 4 \text { sec. } 22, \text { T. } 3 \text { N., R. } 22 \text { E., } \\
\text { Racine County, at local road } \\
0.2 \text { mi east of 90th Street, on the } \\
\text { Milwaukee Railroad tracks, at } \\
\text { Sturtevant, Wis. }\end{array}$ & 1.67 & $\begin{array}{l}\text { Sept. } 2,1972 \\
\text { July } 17,1973 \\
\text { Aug. } 8,1973 \\
\text { Oct. } 25,1973 \\
\text { Sept. 19, 1974 } \\
\text { Sept. 19, } 1975\end{array}$ & $\begin{array}{l}2_{0}^{.68} \\
.05 \text { est. } \\
2_{0}^{.01} \\
.01\end{array}$ & t. & 0 & c \\
\hline DS15 & $\begin{array}{l}\text { Pike River } \\
\text { tributary }\end{array}$ & $\begin{array}{l}\text { SW1/4SE1/4 sec. 9, T. } 2 \text { N., R. } 22 \text { E., } \\
\text { Kenosha County, at culvert on } \\
\text { Chicago and Milwaukee Railroad } \\
\text { at north edge of Somers, Wis. }\end{array}$ & .82 & $\begin{array}{l}\text { Sept. } 2,1972 \\
\text { July } 17,1973 \\
\text { Aug. } 8,1973 \\
\text { Oct. } 26,1973 \\
\text { Sept. } 19,1974 \\
\text { Sept. } 19,1975\end{array}$ & $\begin{array}{l}4_{0}^{0.18} \\
.01 \text { est. } \\
.01 \text { est. } \\
\AA_{0}^{4.01} \text { est. }\end{array}$ & $\begin{array}{l}\text { t. } \\
\text { t. } \\
\text { t. }\end{array}$ & 0 & c \\
\hline DS16 & Pike Creek & $\begin{array}{l}\text { NE } 1 / 4 \text { SE } 1 / 4 \text { sec. } 25, \text { T. } 2 \text { N., R. } 22 \text { E., } \\
\text { Kenosha County, just upstream from } \\
\text { golf course tributary, } 1.4 \text { mi } \\
\text { northwest of post office in } \\
\text { Kenosha, Wis. }\end{array}$ & 2.42 & $\begin{array}{l}\text { Sept. } 19,1974 \\
\text { Sept. } 19,1975 \\
\text { Oct. } 20,1977 \\
\text { Sept. } 6,1978\end{array}$ & $\begin{array}{l}.05 \\
.14 \\
.15 \\
.27\end{array}$ & .02 & $<.01$ & c \\
\hline DS20 & $\begin{array}{l}\text { Carol Beach } \\
\text { tributary }\end{array}$ & $\begin{array}{l}\text { SWY/4NEY/4 sec. 30, T. } 1 \text { N., R. } 23 \text { E., } \\
\text { Kenosha County, at culvert on } \\
\text { Sheridan Road near Sheridan Road } \\
\text { School, at Carol Beach, Wis. }\end{array}$ & 1.97 & $\begin{array}{l}\text { Sept. } 2,1972 \\
\text { July } 18,1973 \\
\text { Aug. } 8,1973 \\
\text { Sept. 19, } 1974 \\
\text { Sept. 19, } 1975\end{array}$ & $\begin{array}{l}1.20 \\
.10 \\
.02 \\
.03 \\
.01\end{array}$ & $<.01$ & $<.01$ & c \\
\hline DS1A & $\begin{array}{l}\text { Tributary to } \\
\text { Des Plaines } \\
\text { River tributary }\end{array}$ & $\begin{array}{l}\text { NE } 1 / 4 \text { NW } 1 / 4 \text { sec. } 17, \text { T. } 1 \text { N., R. } 22 \text { E., } \\
\text { Kenosha County, at Bain Station } \\
\text { Road, at Pleasant Prairie, Wis. }\end{array}$ & .18 & $\begin{array}{l}\text { Sept. 19, } 1974 \\
\text { Sept. 19, } 1975 \\
\text { Oct. 20, } 1977\end{array}$ & $\begin{array}{l}{ }_{2}^{2} \\
2_{0}^{0} \\
{ }_{0}^{0}\end{array}$ & 0 & 0 & c \\
\hline
\end{tabular}

1Based on 1967 report by Wisconsin Department of Natural Resources (DNR). Site lettered " $A$ " was not included in 1967 DNR report.

2 Streambed is dry.

${ }^{4}$ Negligible discharge--water ponded, unable to measure velocity. 
Table 7. Duck Creek-Pensaukee River basin station location and low-flow characteristics.
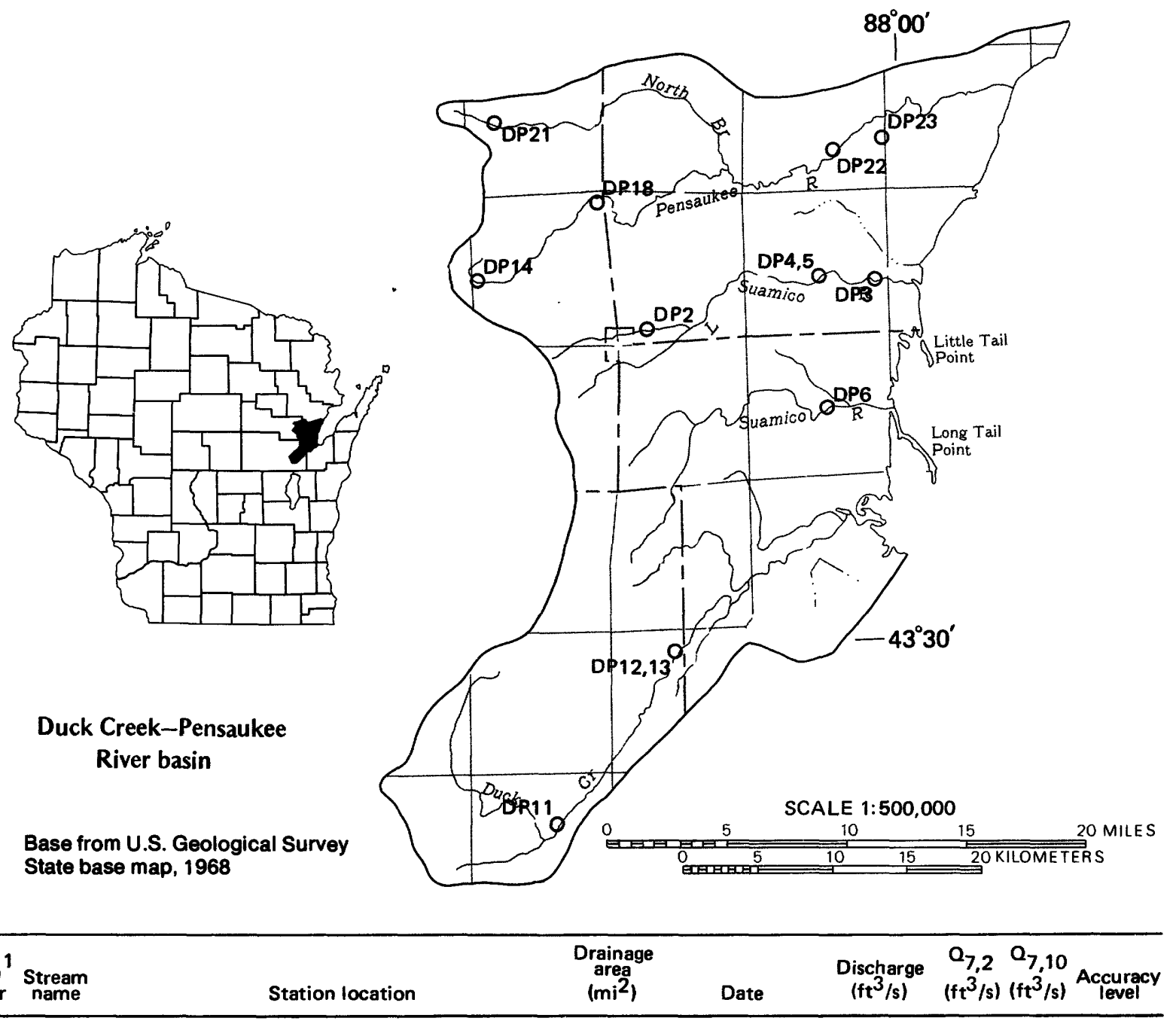

\section{Duck Creek-Pensaukee River basin}

Base from U.S. Geological Survey State base map, 1968

\begin{tabular}{|c|c|c|c|c|c|c|}
\hline $\begin{array}{l}\text { Station } \\
\text { r.umber }\end{array}$ & $\underset{\text { Stream }}{\text { name }}$ & Station location & $\begin{array}{c}\text { Drainage } \\
\text { area } \\
\left(\mathrm{mi}^{2}\right)\end{array}$ & Date & $\begin{array}{c}\text { Discharge } \\
\left(\mathrm{ft}^{3} / \mathrm{s}\right)\end{array}$ & 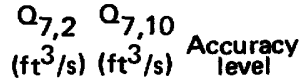 \\
\hline
\end{tabular}

DP2 Little Suamico River

Little Suamico River

DP3 Little Suamico River

DP4, Little Suamico 5 River
NE $1 / 4$ SW $1 / 4$ sec. 32 , T. 26 N., R. 19 E., Oconto County, at sewage-treatment plant, 1.0 mi northeast of Pulaski, Wis.

NW1/4NE1/4 sec. 33, T. 26 N., R. 19 E. Oconto River, at culvert on Jaworski Road, 2.2 mi northeast of Pulaski. Wis.

SE $1 / 4$ SE$^{1 / 4}$ sec. 24, T. 26 N., R. 20 E., Oconto River, at bridge on County Trunk J, $0.3 \mathrm{mi}$ south of Little Suamico, Wis.

SE $1 / 4$ SE $1 / 4$ sec. 21, T. 26 N., R. 20 E., Oconto River, at bridge on Cross Road, $0.7 \mathrm{mi}$ south of Sobieski, Wis.

\subsection{6}

9.29 July 27,1972 Oct. 17, 1972

Aug. 2, 1973

Oct. 24,1975

July 14,1976

Oct. 14,1976

54.8 Aug. 13, 1969

Aug. 28, 1969

Oct. 17, 1972

July 8,1976

July 14,1976

Oct. 14, 1976

41.1 Aug. 13, 1969

Aug. 28, 1969

July 27, 1972

Oct. 17, 1972

Aug. 3, 1973

Oct. 24, 1975

July 14, 1976

Oct. 14, 1976
0.72

.47

.34

.52

.25

.22

1.46

66

9.73

1.87

2.14

1.35

3.36

5.39

3.02

6.42

.55

1.86 
Table 7. Duck Creek-Pensaukee River basin station location and low-flow characteristics-Continued.

\begin{tabular}{|c|c|c|c|c|c|c|}
\hline $\begin{array}{l}\text { Station }{ }^{1} \\
\text { number }\end{array}$ & $\underset{\text { name }}{\text { Stream }}$ & Station location & $\begin{array}{l}\text { Drainage } \\
\text { area } \\
\left(\mathrm{mi}^{2}\right)\end{array}$ & Date & $\begin{array}{c}\text { Discharge } \\
\left(\mathrm{ft}^{3} / \mathrm{s}\right)\end{array}$ & $\underset{\left(\mathrm{ft}^{3} / \mathrm{s}\right)\left(\mathrm{ft}^{3} / \mathrm{s}\right)}{\mathrm{Q}_{7,2}} \mathrm{Q}_{7,10}$ Accuracy \\
\hline
\end{tabular}

DP6 Suamico River

Suamico Rivar

Duck Creek

DP12, Duck Creek 13

Duck Creek

DP14 Pensaukee River

Pensaukee River

DP18 Pensaukee River

DP21 North Branch Pensaukee River

DP22 Pensaukee River
NE $1 / 2$ SW $1 / 4$ sec. 21, T. 25 N., R. 20 E. Brown County, at bridge on country road, $1.5 \mathrm{mi}$ southwest of Suamico, Wis.

NW1/4 sec. 22, T. 25 N., R. 20 E., Brown County, at bridge on Country Trunk B, $0.5 \mathrm{mi}$ west of Suamico, $7.5 \mathrm{mi}$ north of Green Bay, $3.0 \mathrm{mi}$ upstream from mouth.

NW $1 / 2 N E \% / /$ sec. 21, T. 22 N., R. 18 E., Outagamie County, at sewage-treatment plant facility for grade school of Freedom School District No. 3 $2.0 \mathrm{mi}$ southwest of Freedom, Wis.

SE $1 / 2 S E 1 / 4$ sec. 16, T. 22 N., R. 18 E. Outagamie County, at bridge on Vine Road, $1.4 \mathrm{mi}$ southwest of Freedom, Wis.

NW $1 / 4$ SE $1 / 4$ sec. 17, T. 23 N., R. 19 E., Outagamie County, at town road, 2.9 mi southwest of Oneida, Wis.

SW $1 / 4$ NW $1 / 1$ sec. 3, T. 23 N., R. 19 E. Brown County, at sewage-treatment facility, $0.3 \mathrm{mi}$ upstream from Stete Highway 54 bridge, at Oneida, Wis.

SW $\%$ SW $1 / 4$ sec. 7, T. 26 N., R. 18 E., Shawano County, at bridge on town road, at Zachow, Wis.

SE $1 / 4 N E 1 / 4$ sec. 24, T. 26 N., R. 17 E., Shawano County, at bridge on County Trunk F, $1.3 \mathrm{mi}$ south of Zachow, Wis.

NE $1 / / N E 1 / 4$ sec. 1, T. 26 N., R. 18 E. Shawano County, at State Highway 32 at Krakow, $6.3 \mathrm{mi}$ north of

Pulaski, Wis.

SE $1 / 4 S E 1 / 4$ sec. 18, T. 27 N., R. 18 E. Shawano County, at bridge on country road, $1.8 \mathrm{mi}$ northwest of Advance, Wis.

NW $1 / 4 W 1 / 4$ sec. 26, T. 27 N., R. 20 E., Oconto County, at bridge on U.S. Highway 141, 1.1 mi northeast of Abrams, Wis.

56.7

57.0 July 27,1972

Oct. 17, 1972

Aug. 3,1973

June 27,1974

Oct. 24, 1975

8.72

45.3

Oct. 17,1972

Oct. 24, 1975

Julv 14, 1976

92.2

July 26, 1972

Aug. 2, 1973

Oct. 24, 1975

Oct. 30,1975

Oct. 14, 1976

July 27, 1977

94.1

3.73

8.05

July 27, 1972

Oct. 18, 1972

Aug. 2, 1973

Oct. 23, 1975

July 14,1976

Oct. 14, 1976

41.8 Julv 27,1972

Oct. 18, 1972

Aug. 2, 1973

Oct. 23, 1975

Julv 14, 1976

Oct. 14, 1976

July 27, 1977

5.17 Oct. 23, 1975

July 14, 1976

Oct. 14, 1976

115

July 27, 1972

Oct. 18, 1972

Aug. 2, 1973

Oct. 23, 1975

July 14, 1976

Oct. 14, 1976

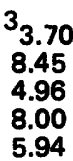

$\begin{array}{ll}1.6 & 0.88\end{array}$

b

8.00

5.94

4.53

$\sum_{0}^{.05}$ est.

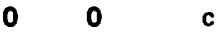

3.38

1.24

.85

.92

$2_{0}^{19}$
.67

.65

1.32

1.38

.73

.51

30.81

3.54

1.88

3.56

.30

.51

.13

${ }_{2}^{4} 0$

7.18

20.0

8.99

14.7

4.06

4.56
$.11 \quad .03 \quad c$

$.19 \quad .05 \quad b$

$\begin{array}{lll}2.8 & 1.0 & c\end{array}$ 
Table 7. Duck Creek-Pensaukee River basin station location and low-flow characteristics-Continued.

\begin{tabular}{|c|c|c|c|c|c|c|}
\hline $\begin{array}{l}\text { Station } \\
\text { number }\end{array}$ & Station location & $\begin{array}{c}\text { Drainage } \\
\text { area } \\
\left(\mathrm{mi}^{2}\right)\end{array}$ & Date & $\begin{array}{c}\text { Discharge } \\
\left(\mathrm{ft}^{3} / \mathrm{s}\right)\end{array}$ & $\begin{array}{r}0_{7,2} \quad a_{7,10} \\
\left(\mathrm{ft}^{3} / \mathrm{s}\right)\left(\mathrm{ft}^{3} / \mathrm{s}\right)\end{array}$ & $\begin{array}{c}\text { Accuracy } \\
\text { level }\end{array}$ \\
\hline DP23 Brookside Creek & $\begin{array}{l}\text { NE1/4NW1/4 sec. } 25, \text { T. } 27 \text { N., R. } 20 \text { E., } \\
\text { Oconto County, at culvert on country } \\
\text { road, } 1.6 \text { mi southwest of Brookside, } \\
\text { Wis. }\end{array}$ & 1.80 & $\begin{array}{l}\text { Oct. } 23,1975 \\
\text { July } 14,1976 \\
\text { Oct. 14, } 1976 \\
\text { July } 27,1977\end{array}$ & $\begin{array}{l}4.31 \\
<.10 \text { est. } \\
.04 \text { est. }\end{array}$ & $0.05<0.0$ & c \\
\hline
\end{tabular}

${ }^{1}$ Based on 1971 report by Wisconsin Department of Natural Resources.

2 Streambed is dry.

3 Additional discharge measurements are available.

${ }^{4}$ Negligible discharge--water ponded, unable to measure velocity.

7 Unable to define relationship. 
Table 8. Fox River (IIlinois) basin station locations and low-flow characteristics.

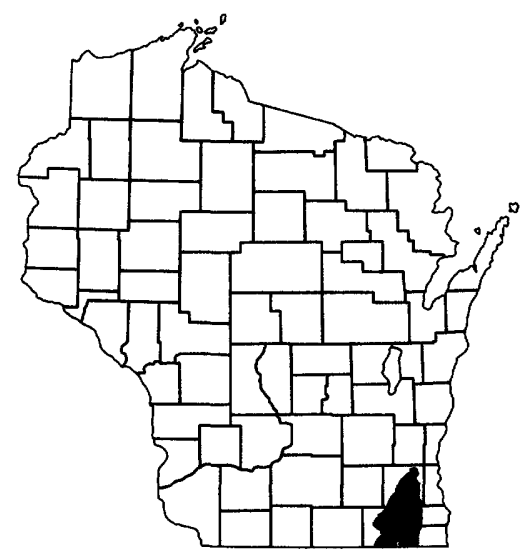

Fox River (Illinois) basin

SCALE 1: 500,000

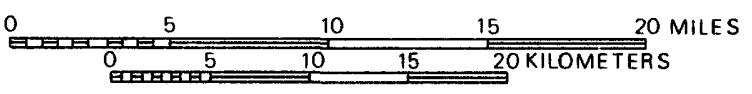

Base from U.S. Geological Survey State base map, 1968

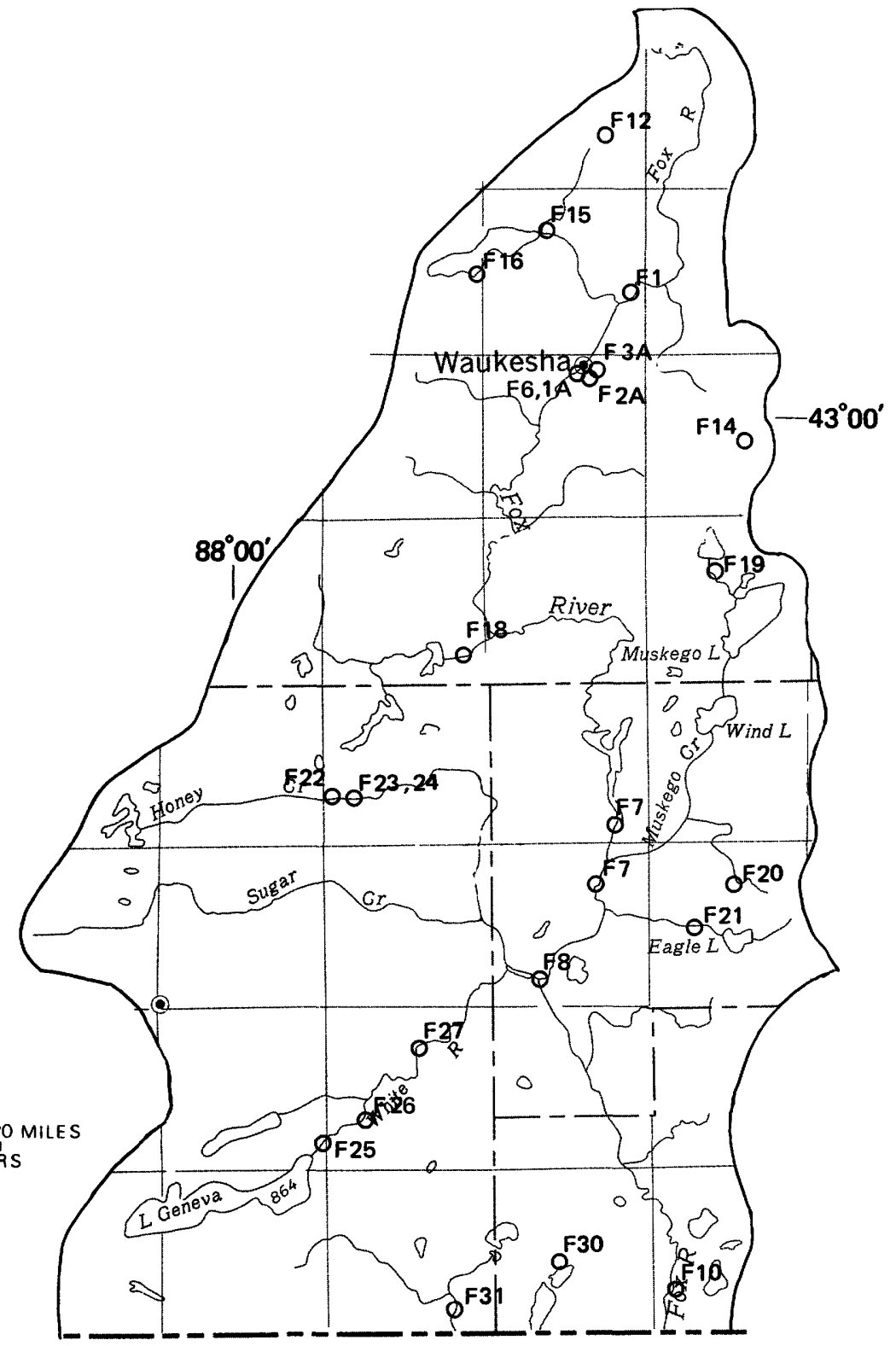

\begin{tabular}{|c|c|c|c|c|c|c|}
\hline $\begin{array}{l}\text { Station } \\
\text { number }\end{array}$ & $\underset{\text { name }}{\text { Stream }}$ & Station location & $\begin{array}{c}\text { Drainage } \\
\text { area } \\
\left(\mathrm{mi}^{2}\right)\end{array}$ & Date & $\begin{array}{c}\text { Discharge } \\
\left(\mathrm{ft}^{3} / \mathrm{s}\right)\end{array}$ & $\begin{array}{l}\mathrm{Q}_{7,2} \mathrm{O}_{7,10} \\
\left(\mathrm{ft}^{3} / \mathrm{s}\right)\left(\mathrm{ft}^{3} / \mathrm{s}\right)\end{array}$ Accuracy \\
\hline
\end{tabular}

F1 Fox River
SWY/4NE $/ 4$ sec. 19, T. 7 N., R. 20 E., Waukesha County, at sewage-disposal ponds, just downstream from Poplar River, 2.3 mi west of city hall in Brookfield, Wis.

NE $1 / 4$ sec. 24, T. 7 N., R. 19 E., Waukesha County, at bridge on County Trunk SS, 3.5 mi northeast of Waukesha, Wis.
74.1

77.4 $\begin{array}{lll}4.0 & 2.0 \quad b\end{array}$ 
Table 8. Fox River (Illinois) basin station locations and low-flow characteristics-Continued.

\begin{tabular}{|c|c|c|c|c|c|c|c|c|}
\hline $\begin{array}{l}\text { Station } \\
\text { number }\end{array}$ & 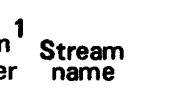 & Station location & $\begin{array}{l}\text { Drainage } \\
\text { area } \\
\left(\mathrm{mi}^{2}\right)\end{array}$ & Date & $\begin{array}{c}\text { Discharge } \\
\left(\mathrm{ft}^{3} / \mathrm{s}\right)\end{array}$ & $\begin{array}{l}Q_{7,2} Q_{7} \\
\left(\mathrm{ft}^{3 / \mathrm{s}}\right)\left(\mathrm{ft}^{3}\right.\end{array}$ & & $\begin{array}{l}\text { Accuracy } \\
\text { level }\end{array}$ \\
\hline \multirow[t]{2}{*}{ F6,1A } & Fox River & $\begin{array}{l}\text { SWY/4 sec. 3, T. } 6 \text { N., R. 19 E., } \\
\text { Waukesha County, on left bank, } \\
20 \text { ft downstream from Prairie } \\
\text { Street bridge in Waukesha, } 1.0 \mathrm{mi} \\
\text { downstream from dam, } 3.2 \mathrm{mi} \\
\text { downstream from Pewaukee River. }\end{array}$ & 126 & & & 12 & 4.4 & $\mathbf{a}$ \\
\hline & Fox River & $\begin{array}{l}\text { SW1/4NE1/4 sec. } 9, \text { T. } 6 \text { N., R. } 19 \text { E., } \\
\text { Waukesha County, at sewage-treatment } \\
\text { plant, } 1.6 \text { mi southwest of post } \\
\text { office in Waukesha, Wis. }\end{array}$ & 129 & & & & & \\
\hline \multirow[t]{2}{*}{ F7 } & Fox River & $\begin{array}{l}\text { NE } 1 / \text { SE } 1 / 4 \text { sec. } 35, \text { T. } 4 \text { N., R. } 19 \text { E.. } \\
\text { Racine County, at bridge on State } \\
\text { Highways } 20 \text { and } 36 \text {, in Waterford, } \\
\text { Wis. }\end{array}$ & 357 & $\begin{array}{l}\text { Sept. } 13,1966 \\
\text { Nov. } 29,1972 \\
\text { Aug. } 7,1973 \\
\text { May } 21,1975 \\
\text { July } 10,1975 \\
\text { Sept. } 16,1975\end{array}$ & $\begin{array}{l}48.7 \\
328 \\
147 \\
364 \\
213 \\
132\end{array}$ & 32 & 16 & c \\
\hline & Fox River & $\begin{array}{l}\text { SW\%NW1/4 sec. 11, T. } 3 \text { N., R. } 19 \text { E., } \\
\text { Racine County, } 0.4 \text { mi south of } \\
\text { State Highway } 189 \text { bridge, in } \\
\text { Rochester, Wis. }\end{array}$ & 447 & $\begin{array}{l}\text { Sept. } 17,1975 \\
\text { Oct. } 19,1977 \\
\text { Aug. } 16,1978\end{array}$ & $\begin{array}{l}142 \\
23.6 \\
121\end{array}$ & 7 & $\underline{7}$ & \\
\hline F8 & Fox River. & $\begin{array}{l}\text { NW1/4NW1/4 sec. 4, T. } 2 \text { N., R. } 19 \text { E., } \\
\text { Racine County, at sewage-treatment } \\
\text { plant, at Burlington, Wis. }\end{array}$ & 770 & $\begin{array}{l}\text { Sept. } 18,1974 \\
\text { Sept. } 16,1975 \\
\text { Oct. } 19,1977\end{array}$ & $\begin{array}{l}226 \\
233 \\
125\end{array}$ & 70 & 42 & c \\
\hline \multirow[t]{2}{*}{ F10 } & Fox River & $\begin{array}{l}\text { NE } 1 / 4 N E 1 / 4 \text { sec. } 19, \text { T. } 1 \text { N., R. } 20 \text { E., } \\
\text { Kenosha County, just downstream } \\
\text { from Silver Lake tributary. } \\
0.7 \text { mi south of Silver Lake, Wis. }\end{array}$ & 865 & & & & & \\
\hline & Fox River & $\begin{array}{l}\text { SW1/ sec. } 30, \mathrm{~T} .1 \mathrm{~N} ., \mathrm{R} .20 \mathrm{E} . \text {, } \\
\text { Kenosha County, on right bank } \\
100 \mathrm{ft} \text { downstream from bridge on } \\
\text { County Trunk C, } 300 \mathrm{ft} \text { upstream } \\
\text { from Wilmot Dam, } 1.0 \mathrm{mi} \text { north of } \\
\text { Wisconsin-lllinois State line, } \\
6.0 \text { mi upstreem from Fox chain } \\
\text { of lakes. }\end{array}$ & 868 & & & 100 & 60 & $\mathbf{a}$ \\
\hline \multirow[t]{2}{*}{ F12 } & $\begin{array}{l}\text { Fox River } \\
\text { tributary }\end{array}$ & $\begin{array}{l}\text { NEY } 1 / 2 E 1 / 4 \text { sec. } 26, T .8 \text { N., R. } 19 \text { E., } \\
\text { Waukesha County, at bridge on } \\
\text { County Trunk VV, at Sussex, Wis. }\end{array}$ & 7.68 & $\begin{array}{l}\text { June } 8,1972 \\
\text { July } 17,1973 \\
\text { Aug. } 8,1973 \\
\text { Sept. } 16,1974 \\
\text { Sept. } 15,1975 \\
\text { Oct. } 18,1977\end{array}$ & $\begin{array}{l}.72 \\
.62 \\
.24 \\
.51 \\
1.80 \\
1.21\end{array}$ & .11 & .03 & c \\
\hline & $\begin{array}{l}\text { Fox River } \\
\text { tributary }\end{array}$ & $\begin{array}{l}\text { NE1/4SW// sec. } 26, \text { T. } 8 \text { N., R. } 19 \text { E., } \\
\text { Waukesha County, at sewage-treatment } \\
\text { plant, at Sussex, Wis. }\end{array}$ & 7.81 & & & & & \\
\hline F14 & $\begin{array}{l}\text { Poplar Creek } \\
\text { tributary }\end{array}$ & $\begin{array}{l}\text { NW1/4NE1/4 sec. 23, T. } 6 \text { N., R. } 20 \text { E., } \\
\text { Waukesha County, } 0.8 \text { mi upstreem } \\
\text { from County Trunk ES, just downstream } \\
\text { from storm sewer outlet at end of } \\
\text { Hargrove Street, in New Berlin, Wis. }\end{array}$ & .93 & $\begin{array}{l}\text { Sept. 15, } 1975 \\
\text { Oct. 18, } 1977 \\
\text { Aug. } 15,1978\end{array}$ & $\begin{array}{l}.02 \\
.36 \\
.06\end{array}$ & $<.01$ & $<.01$ & c \\
\hline F15 & $\begin{array}{l}\text { Pewaukee } \\
\text { River }\end{array}$ & $\begin{array}{l}\text { SW1/4SE1/4 sec. 9, T. } 7 \text { N., R. } 19 \text { E., } \\
\text { Waukesha County, at sewage-treatment } \\
\text { plant, at Pewaukee, Wis. }\end{array}$ & 33.0 & $\begin{array}{l}\text { June } 8,1972 \\
\text { July } 17,1973 \\
\text { Aug. } 8,1973 \\
\text { Sept. 16, 1974 } \\
\text { Sept. 15, } 1975\end{array}$ & $\begin{array}{l}15.0 \\
6.97 \\
3.09 \\
3.00 \\
7.65\end{array}$ & .87 & .18 & c \\
\hline F16 & Zion Creek & $\begin{array}{l}\text { SEYNWW/ sec. } 24, T .7 \text { N., R. } 18 \text { E., } \\
\text { Waukesha County, at culvert on } \\
\text { Oakton Road, } 3.1 \text { mi southwest of } \\
\text { Pewaukee, Wis. }\end{array}$ & 3.40 & $\begin{array}{l}\text { Sept. } 16,1974 \\
\text { Sept. } 15,1975 \\
\text { Oct. } 18,1977 \\
\text { Aug. } 15,1978\end{array}$ & $\begin{array}{l}1.26 \\
.82 \\
.91 \\
.64\end{array}$ & .20 & .06 & c \\
\hline
\end{tabular}


Table 8. Fox River (Illinois) basin station locations and low-flow characteristics-Continued.

\begin{tabular}{|c|c|c|c|c|c|c|c|c|}
\hline $\begin{array}{l}\text { Station } \\
\text { numbe }\end{array}$ & $\mathrm{n}^{1} \underset{\text { name }}{\text { Stream }}$ & Station location & $\begin{array}{l}\text { Drainage } \\
\text { area } \\
\left(\mathrm{mi}^{2}\right)\end{array}$ & Date & $\begin{array}{c}\text { Discharge } \\
\left(\mathrm{ft}^{3} / \mathrm{s}\right)\end{array}$ & $\begin{array}{l}\mathrm{Q}_{7,2} \\
\left(\mathrm{ft} \mathrm{t}^{3} / \mathrm{s}\right)(\mathrm{ft}\end{array}$ & $\left.t^{3} / \mathrm{s}\right)$ & $\begin{array}{l}\text { Accuracy } \\
\text { level }\end{array}$ \\
\hline \multirow[t]{2}{*}{ F18 } & $\begin{array}{l}\text { Mukwonago } \\
\text { River }\end{array}$ & $\begin{array}{l}\text { NE1/4NE1/4 sec. } 35, \text { T. } 5 \text { N., R. } 18 \text { E., } \\
\text { Waukesha County, on U.S. Highway } \\
83,0.6 \text { mi south of Mukwonago, Wis. }\end{array}$ & 74.0 & $\begin{array}{l}\text { June } 28,1972 \\
\text { July } 19,1973 \\
\text { Aug. } 7,1973\end{array}$ & $\begin{array}{r}332.2 \\
25.6 \\
97.7\end{array}$ & $\underline{6}$ & $\underline{6}$ & \\
\hline & $\begin{array}{l}\text { Mukwonago } \\
\text { River }\end{array}$ & $\begin{array}{l}\text { SW } 1 / 4 \text { SW } 1 / 4 \text { sec. } 25, \text { T. } 5 \text { N., R. } 18 \mathrm{E} \text {., } \\
\text { Waukesha County, at sewage-treatment } \\
\text { plant, at Mukwonago, Wis. }\end{array}$ & 74.3 & & & & & \\
\hline F19 & Muskego Creek & $\begin{array}{l}\mathrm{SE} \mathrm{y}^{1} \mathrm{SW} 1 / 4 \text { sec. } 9, \mathrm{~T} .5 \mathrm{~N} ., \mathrm{R} .20 \mathrm{E} ., \\
\text { Waukesha County, at bridge on } \\
\text { town road, at Muskego, Wis. }\end{array}$ & 11.9 & $\begin{array}{l}\text { June } 8,1972 \\
\text { July } 19,1973 \\
\text { Aug. } 7,1973 \\
\text { Sept. 17, 1974 } \\
\text { Sept. 15, 1975 } \\
\text { Oct. } 18,1977 \\
\text { Aug. } 15,1978\end{array}$ & $\begin{array}{c}3.02 \\
1.63 \\
1.55 \\
11.3 \\
.57 \\
3.39 \\
1.43\end{array}$ & $\underline{6}$ & 6 & \\
\hline F2O & $\begin{array}{l}\text { Tributary to } \\
\text { Goose Lake } \\
\text { Branch Canal } \\
\text { tributary }\end{array}$ & $\begin{array}{l}\text { NE } 1 / 4 \text { NW } 1 / 4 \text { sec. } 9, \text { T. } 3 \text { N., R. } 20 \text { E., } \\
\text { Racine County, at culvert on } \\
\text { State Highway } 20,4.1 \text { mi } \\
\text { east of Rochester, Wis. }\end{array}$ & 2.69 & $\begin{array}{l}\text { Sept. } 17,1974 \\
\text { Sept. 17, } 1975 \\
\text { Oct. } 19,1977\end{array}$ & $\begin{array}{c}.04 \\
4.01 \\
0\end{array}$ & 0 & 0 & c \\
\hline F21 & Eagle Creek & $\begin{array}{l}\text { NW1/4NW1/4 sec. } 21, T .3 \text { N., R. } 20 \text { E., } \\
\text { Racine County, at culvert on County } \\
\text { Trunk N, } 4.3 \text { mi southeast of } \\
\text { Rochester, Wis. }\end{array}$ & 8.58 & $\begin{array}{l}\text { Sept. } 17,1974 \\
\text { Sept. 17, } 1975 \\
\text { Oct. 19, } 1977\end{array}$ & $\begin{array}{r}<.01 \\
.01 \\
.01\end{array}$ & $<.01$ & $<.01$ & c \\
\hline F22 & Honey Creek & $\begin{array}{l}\text { SE } 1 / 4 N^{\prime} E^{1 / 4} \text { sec. } 25, \text { T. } 4 \text { N., R. } 17 \text { E., } \\
\text { Walworth County, at culvert on } \\
\text { town road, } 1.1 \text { mi west of } \\
\text { East Troy, Wis. }\end{array}$ & 40.9 & $\begin{array}{l}\text { Nov. } 3,1965 \\
\text { Sept. } 17,1974 \\
\text { Sept. } 17,1975 \\
\text { Oct. } 19,1977\end{array}$ & $\begin{array}{l}21.7 \\
24.5 \\
19.7 \\
14.3\end{array}$ & 6.6 & 4.6 & c \\
\hline \multirow[t]{2}{*}{$\begin{array}{l}\text { F23, } \\
24\end{array}$} & Honey Creek & $\begin{array}{l}\text { SW1/4NW1/4 sec. } 29, \text { T. } 4 \text { N., R. } 18 \text { E., } \\
\text { Walworth County, at bridge on } \\
\text { County Trunk G, at East Troy, Wis. }\end{array}$ & 44.2 & $\begin{array}{l}\text { June } 8,1972 \\
\text { July } 19,1973 \\
\text { Aug. } 7,1973 \\
\text { Sept. } 17,1974 \\
\text { Sept. } 16,1975 \\
\text { Oct. } 18,1977\end{array}$ & $\begin{array}{l}9.59 \\
20.1 \\
27.2 \\
16.2 \\
16.4 \\
13.3\end{array}$ & 7 & $\underline{7}$ & \\
\hline & Honey Creek & $\begin{array}{l}\text { NW1/4NE } 1 / 4 \text { sec. } 29, \text { T. } 4 \text { N., R. } 18 \text { E., } \\
\text { Walworth County, at sewage- } \\
\text { treatment plant, at East Troy, } \\
\text { Wis. }\end{array}$ & 45.2 & & & & & \\
\hline F25 & White River & $\begin{array}{l}\text { SE } 1 / 4 \mathrm{SE}^{1} / 4 \text { sec. } 25, \mathrm{~T} .2 \mathrm{~N} ., \mathrm{R} .17 \mathrm{E.} \\
\text { Walworth County, at sewage- } \\
\text { treatment plant, at Lake Geneva, } \\
\text { Wis. }\end{array}$ & 29.5 & $\begin{array}{l}\text { Sept. } 12,1966 \\
\text { Aug. } 15,1967 \\
\text { June } 7,1972 \\
\text { June } 26,1972 \\
\text { Nov. } 28,1972 \\
\text { July 19, } 1973 \\
\text { Aug. } 9,1973 \\
\text { Sept. 18, } 1974 \\
\text { Sept. 17, } 1975 \\
\text { Oct. } 19,1977\end{array}$ & $\begin{array}{l}1.69 \\
12.4 \\
16.0 \\
29.3 \\
15.4 \\
8.53 \\
28.2 \\
3.36 \\
6.19 \\
2.05\end{array}$ & 1.7 & .89 & c \\
\hline F26 & White River & $\begin{array}{l}\text { NEY } 1 / 4 W 1 / 4 \text { sec. } 29, \text { T. } 2 \text { N., R. } 18 \text { E., } \\
\text { Walworth County, at bridge on private } \\
\text { road, } 2.3 \text { mi northeast of post } \\
\text { office in Lake Geneva, Wis. }\end{array}$ & 38.9 & $\begin{array}{l}\text { Sept. } 18,1974 \\
\text { Sept. 17, } 1975 \\
\text { Oct. 19, } 1977\end{array}$ & $\begin{array}{l}8.78 \\
11.0 \\
5.71\end{array}$ & 2.6 & 1.3 & c \\
\hline F27 & White River & $\begin{array}{l}\text { SW } 1 / 4 N E 1 / 4 \text { sec. } 10, T .2 \text { N., R. } 18 \text { E., } \\
\text { Walworth County, just downstream } \\
\text { from tail race of mill pond dam, } \\
\text { at Lyons, Wis. }\end{array}$ & 81.4 & $\begin{array}{l}\text { June } 7,1972 \\
\text { June } 26,1972 \\
\text { July } 19,1973 \\
\text { Aug. } 9,1973 \\
\text { Sept. 18, } 1974 \\
\text { Sept. 17, } 1975\end{array}$ & $\begin{array}{l}36.0 \\
44.4 \\
24.5 \\
40.7 \\
16.1 \\
16.5\end{array}$ & 7.0 & 3.7 & c \\
\hline
\end{tabular}


Table 8. Fox River (Illinois) basin station locations and low-flow characteristics-Continued.

\begin{tabular}{|c|c|c|c|c|c|c|c|c|}
\hline $\begin{array}{l}\text { Station } \\
\text { numbe }\end{array}$ & $\begin{array}{l}n^{1} \\
\text { er Stream } \\
\text { name }\end{array}$ & Station location & $\begin{array}{l}\text { Drainage } \\
\text { area } \\
\left(\mathrm{mi}^{2}\right)\end{array}$ & Date & $\begin{array}{l}\text { Discharge } \\
\left(\mathrm{ft}^{3} / \mathrm{s}\right)\end{array}$ & $\begin{array}{l}\mathrm{O}_{7,2} \mathrm{Q}_{7} \\
\left(\mathrm{ft}^{3} / \mathrm{s}\right)\left(\mathrm{ft}^{3}\right.\end{array}$ & $3 / 5)$ & $\underset{\text { level }}{\text { Accuracy }}$ \\
\hline \multirow[t]{2}{*}{ F30 } & $\begin{array}{l}\text { Bassett Creek } \\
\text { tributary }\end{array}$ & $\begin{array}{l}\text { NEY1/4NW1/4 sec. 22, T. } 1 \text { N., R. } 19 \text { E., } \\
\text { Kenosha County, at sewage-treatment } \\
\text { plant, at Twin Lakes, Wis. }\end{array}$ & .51 & & & & & \\
\hline & Bassett Creek & $\begin{array}{l}\text { SE } 1 / 4 \text { SE } 1 / 4 \text { sec. } 15, T .1 \text { N., R. } 19 \text { E., } \\
\text { Kenosha County, at bridge on } \\
\text { County Trunk F, } 1.3 \text { mi northeast } \\
\text { of Twin Lakes, Wis. }\end{array}$ & 5.09 & $\begin{array}{l}\text { June } 7,1972 \\
\text { July } 18,1973 \\
\text { Aug. } 9,1973 \\
\text { Sept. 18, 1974 } \\
\text { Sept. 17, } 1975\end{array}$ & $\begin{array}{l}0.68 \\
1.14 \\
1.22 \\
1.20 \\
1.44\end{array}$ & 0.200 & 0.10 & c \\
\hline $\mathbf{F 3 1}$ & $\begin{array}{l}\text { North Branch } \\
\text { Nippersink } \\
\text { Creek }\end{array}$ & $\begin{array}{l}\text { SW } 1 / 4 \text { SE } 1 / 4 \text { sec. } 35, \text { T. } 1 \text { N., R. } 18 \text { E., } \\
\text { Walworth County, at bridge on } \\
\text { County Trunk B, at Genoa City, } \\
\text { Wis. }\end{array}$ & 43.0 & $\begin{array}{l}\text { June } 7,1972 \\
\text { June } 26,1972 \\
\text { July } 18,1973 \\
\text { Aug. } 9,1973 \\
\text { Sept. } 18,1974 \\
\text { Sept. } 17,1975\end{array}$ & $\begin{array}{l}22.1 \\
40.3 \\
31.5 \\
28.9 \\
23.0 \\
17.6\end{array}$ & 6.0 & 3.7 & c \\
\hline F2A & $\begin{array}{l}\text { Fox River } \\
\text { tributary }\end{array}$ & $\begin{array}{l}\text { NE } 1 / 4 N^{1} / / 4 \text { sec. } 2, T .6 \text { N., R. } 19 \text { E., } \\
\text { Waukesha County, at culvert on } \\
\text { State Highway } 59,1.2 \text { mi east } \\
\text { of post office in Waukesha, Wis. }\end{array}$ & 0.74 & $\begin{array}{l}\text { Sept. } 17,1974 \\
\text { Sept. } 15,1975 \\
\text { Oct. } 18,1977 \\
\text { Aug. } 15,1978\end{array}$ & $\begin{array}{l}.51 \\
.55 \\
.73 \\
.49\end{array}$ & .22 & .10 & c \\
\hline F3A & $\begin{array}{l}\text { Fox River } \\
\text { tributary }\end{array}$ & $\begin{array}{l}\text { SW1/4SE1/4 sec. } 35, T .7 \text { N., R, } 19 \text { E., } \\
\text { Waukesha County, at culvert on } \\
\text { town road, approximately } 1.0 \mathrm{mi} \\
\text { northeast of post office in } \\
\text { Waukesha, Wis. }\end{array}$ & 1.74 & $\begin{array}{l}\text { Sept. } 17,1974 \\
\text { Sept. } 15,1975 \\
\text { Oct. } 18,1977 \\
\text { Aug. } 15,1978\end{array}$ & $\begin{array}{l}.85 \\
.76 \\
1.04 \\
.69\end{array}$ & .31 & .15 & c \\
\hline
\end{tabular}

1 Based on 1972 report by Wisconsin Department of Natural Resources (DNR). Site lettered "A" was not included in 1972 DNR report.

${ }^{3}$ Additional discharge measurements are available.

${ }^{4}$ Negligible discharge--water ponded, unable to measure velocity.

${ }^{6}$ No estimate possible due to regulation upstream.

${ }^{7}$ Unable to define relationship. 
Table 9. Lower Fox River basin station locations and low-flow characteristics.

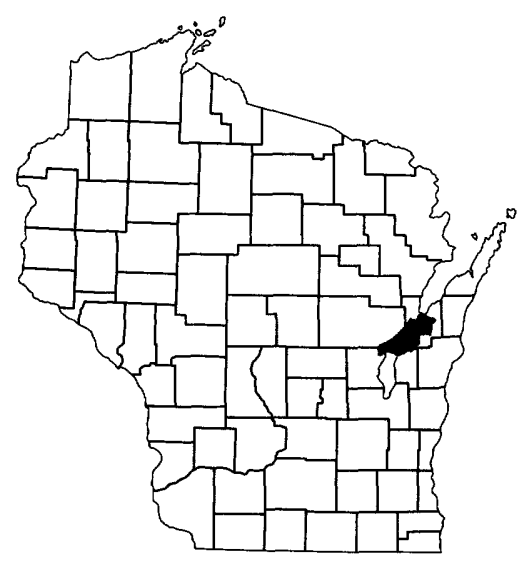

Lower Fox River basin

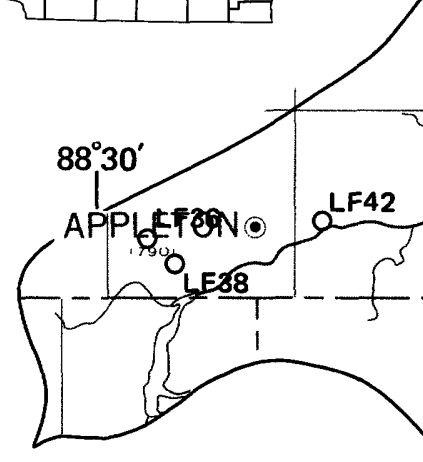

Base from U.S. Geological Survey State base map, 1968

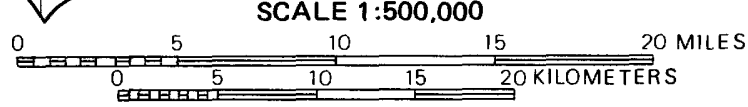

\begin{tabular}{|c|c|c|c|c|c|c|}
\hline $\begin{array}{l}\text { Station }{ }^{1} \\
\text { number }\end{array}$ & $\underset{\substack{\text { Stream } \\
\text { name }}}{\text {. }}$ & Station location & $\begin{array}{l}\text { Drainage } \\
\text { area } \\
\left(\mathrm{mi}^{2}\right)\end{array}$ & Date & $\begin{array}{l}\text { Discharge } \\
\left(\mathrm{ft}^{3} / \mathrm{s}\right)\end{array}$ & 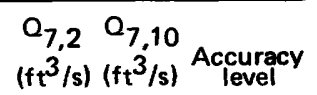 \\
\hline
\end{tabular}

LF36 Mud Creek tributary

Mud Creek

LF38 Mud Creek

LF42 Fox River tributary

LF44, Plum Creek 45 tributary

Plum Creek
SE $1 / 4$ SW $1 / 4$ sec. 20, T. 21 N., R. 17 E., Outagamie County, at bridge on U.S. Highway 10, 3.1 mi west of city hall in Appleton, Wis.

SW $1 / 4$ SE $1 / 4$ sec. 29, T. 21 N., R. 17 E., Outagamie County, 0.1 mi upstream from bridge on County Trunk $V$, $2.8 \mathrm{mi}$ west of city hall in Appleton, Wis.

SE $1 / 4 N W 1 / 4$ sec. 3, T. 20 N., R. 17 E., Winnebago County, 0.1 mi downstream from Chicago and Northwestern Railroad bridge, $2.6 \mathrm{mi}$ southwest of Appleton, Wis.

NE $1 / 4$ NE $1 / 4$ sec. 19, T. 21 N., R. 18 E., Outagamie County, at bridge on U.S. Highway 41, 3.2 mi northeast of post office, in Appleton, Wis.

SE $1 / 4 N W 1 / 4$ sec. 35, T. 21 N., R. 19 E., Brown County, at sewage-treatment plant, at Holland, Wis.

NE $1 / 4$ NW $1 / 4$ sec. 23, T. 21 N., R. 19 E., Brown County, at bridge on country road, $2.4 \mathrm{mi}$ north of Holland, Wis.
0.56

9.9 July 25,1972 Oct. 11, 1972 Aug. 2, 1973 Oct. 30, 1975 July 14, 1976

25.4 July 25, 1972 Oct. 11, 1972

Aug. 2, 1973

Oct. 30, 1975 July 14, 1976

4.81 Oct. 30,1975

July 14, 1976 July 27, 1977

0.08
.94
.06
2.06

.16

1.10

$.02<.01$

c

.16

.12

${ }_{2}^{2}{ }_{2}^{0}$

0

0

c

4.66 July 25,1972

$2_{0}$

0

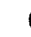

c

19.2 July 25,1972

Oct. 12, 1972

Oct. 31, 1975

July 14, 1976 
Table 9. Lower Fox River basin station locations and low-flow characteristics-Continued.

\begin{tabular}{|c|c|c|c|c|c|c|c|c|c|}
\hline $\begin{array}{l}\text { Station } \\
\text { number }\end{array}$ & ${ }^{1}$ Stream & Station location & $\begin{array}{c}\text { Drainage } \\
\text { ares } \\
\left(\mathbf{m i}^{2}\right)\end{array}$ & Date & $\begin{array}{l}\text { Discharge } \\
\left(\mathrm{ft}^{3} / \mathrm{s}\right)\end{array}$ & $\begin{array}{l}Q_{7,2} \\
\left(\mathrm{ft}^{3} / \mathrm{s}\right)\end{array}$ & $\begin{array}{l}Q_{7} \\
\left(\mathrm{ft}^{3}\right.\end{array}$ & & $\begin{array}{l}\text { ccuracy } \\
\text { level }\end{array}$ \\
\hline \multirow[t]{2}{*}{ LF48 } & $\begin{array}{l}\text { Tributary to } \\
\text { Dutchman Creek } \\
\text { tributary }\end{array}$ & $\begin{array}{l}\text { NE1/aNW1// sec. 7, T. } 23 \text { N., R. } 20 \text { E., } \\
\text { Brown County, at sewage-treatment } \\
\text { plant outfall at Austin-Straubel } \\
\text { Airport, } 0.2 \text { mi upstream from } \\
\text { mouth, } 4.1 \text { mi northwest of post } \\
\text { office at DePere, Wis. }\end{array}$ & 0.90 & & & & & & \\
\hline & $\begin{array}{l}\text { Dutchman Creek } \\
\text { tributary }\end{array}$ & $\begin{array}{l}\text { SW1/aNW1/4 sec. 7, T. } 23 \text { N., R. } 20 \text { E., } \\
\text { Brown County, at culvert on County } \\
\text { Trunk GH, } 3.9 \text { mi northwest of } \\
\text { post office at DePere, Wis. }\end{array}$ & 3.40 & $\begin{array}{l}\text { July } 26,1972 \\
\text { Oct. } 12,1972 \\
\text { Aug. } 2,1973 \\
\text { Oct. } 30,1975 \\
\text { July } 14,1976\end{array}$ & $\begin{array}{l}.10 \\
.98 \\
.06 \\
.15 \\
.08\end{array}$ & & .02 & .01 & c \\
\hline \multirow[t]{2}{*}{ LF52 } & $\begin{array}{l}\text { East Rive, } \\
\text { tributary }\end{array}$ & $\begin{array}{l}\text { SW } 1 / 4 \text { SW } 1 / 4 \text { sec. } 5, \text { T. } 21 \text { N., R. } 20 \text { E., } \\
\text { Brown County, at sewage-treatment } \\
\text { plant, } 0.6 \text { mi west of Greenleaf, } \\
\text { Wis. }\end{array}$ & 7.81 & Aug. 1,1973 & ${ }^{2} 0$ & & $\mathbf{0}$ & 0 & c \\
\hline & East River & $\begin{array}{l}\text { NE1/4SE1/4 sec. } 31, \text { T. } 22 \text { N., R. } 20 \text { E., } \\
\text { Brown County, at bridge on country } \\
\text { road, } 1.7 \text { mi northwest of Greenleaf, } \\
\text { Wis. }\end{array}$ & 37.5 & $\begin{array}{l}\text { Aug. } 13,1969 \\
\text { Aug. } 28,1969 \\
\text { Aug. } 25,1970 \\
\text { July } 25,1972 \\
\text { Oct. } 12,1972 \\
\text { Oct. } 31,1975 \\
\text { July } 14,1976\end{array}$ & $\begin{array}{l}1.25 \\
2.37 \\
.32 \\
2.49 \\
1.42 \\
.02\end{array}$ & & .04 & $<.01$ & c \\
\hline LF1A & $\begin{array}{l}\text { Kankapot Creek } \\
\text { tributary }\end{array}$ & $\begin{array}{l}\text { SE } 1 / \text { NWW } 1 / \text { sec. } 7, \text { T. } 20 \text { N., R. } 19 \text { E., } \\
\text { Calumet County, at culvert on } \\
\text { country road, } 3.4 \text { mi north of } \\
\text { Sherwood, Wis. }\end{array}$ & 1.26 & $\begin{array}{l}\text { Oct. } 31,1975 \\
\text { July 14, } 1976 \\
\text { Sept. 16, } 1976\end{array}$ & $\begin{array}{l}2 \\
2_{0}^{2} \\
2_{0}^{0}\end{array}$ & 0 & 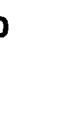 & 0 & c \\
\hline LF2A & East River & $\begin{array}{l}\text { NEY } 1 / 4 N^{1} / 4 \text { sec. } 20, T .22 \text { N., R. } 20 \text { E., } \\
\text { Brown County, at bridge on County } \\
\text { Trunk ZZ, } 4.0 \text { mi north of } \\
\text { Greenleaf, Wis. }\end{array}$ & 49.8 & $\begin{array}{l}\text { Aug. } 13,1969 \\
\text { Aug. } 28,1969 \\
\text { Aug. } 25,1970 \\
\text { Oct. } 30,1975 \\
\text { July } 14,1976 \\
\text { July } 27,1977\end{array}$ & $\begin{array}{l}1.74 \\
4.27 \\
0 \\
2.38 \\
20\end{array}$ & $\mathrm{O}$ & 0 & 0 & c \\
\hline \multicolumn{2}{|c|}{$\begin{array}{l}\text { LF } 3 A \text {, Baird Creek } \\
\text {. }\end{array}$} & $\begin{array}{l}\text { NE } 1 / 4 \text { SW } 1 / \text { sec. } 32, T .24 \text { N., R. } 21 \text { E., } \\
\text { Brown County, at railroad siding } \\
\text { bridge, } 1.6 \text { miles east of court house } \\
\text { in Green Bay, Wis. }\end{array}$ & 27.3 & $\begin{array}{l}\text { Oct. } 30,1975 \\
\text { July } 13,1976 \\
\text { Sept. 16, } 1976 \\
\text { July 26, } 1977\end{array}$ & $\begin{array}{r}1.16 \\
1.78 \\
2.01 \\
.36\end{array}$ & & .54 & .16 & c \\
\hline
\end{tabular}

1 Besed on 1968 report by Wisconsin Department of Natural Resources (DNR). Site lettered "A" was not included in 1968 DNR report.

2 Streambed is dry.

${ }^{4}$ Negligible discharge--water ponded, unable to measure velocity. 
Table 10. Upper Fox River basin station locations and low-flow-characteristics.
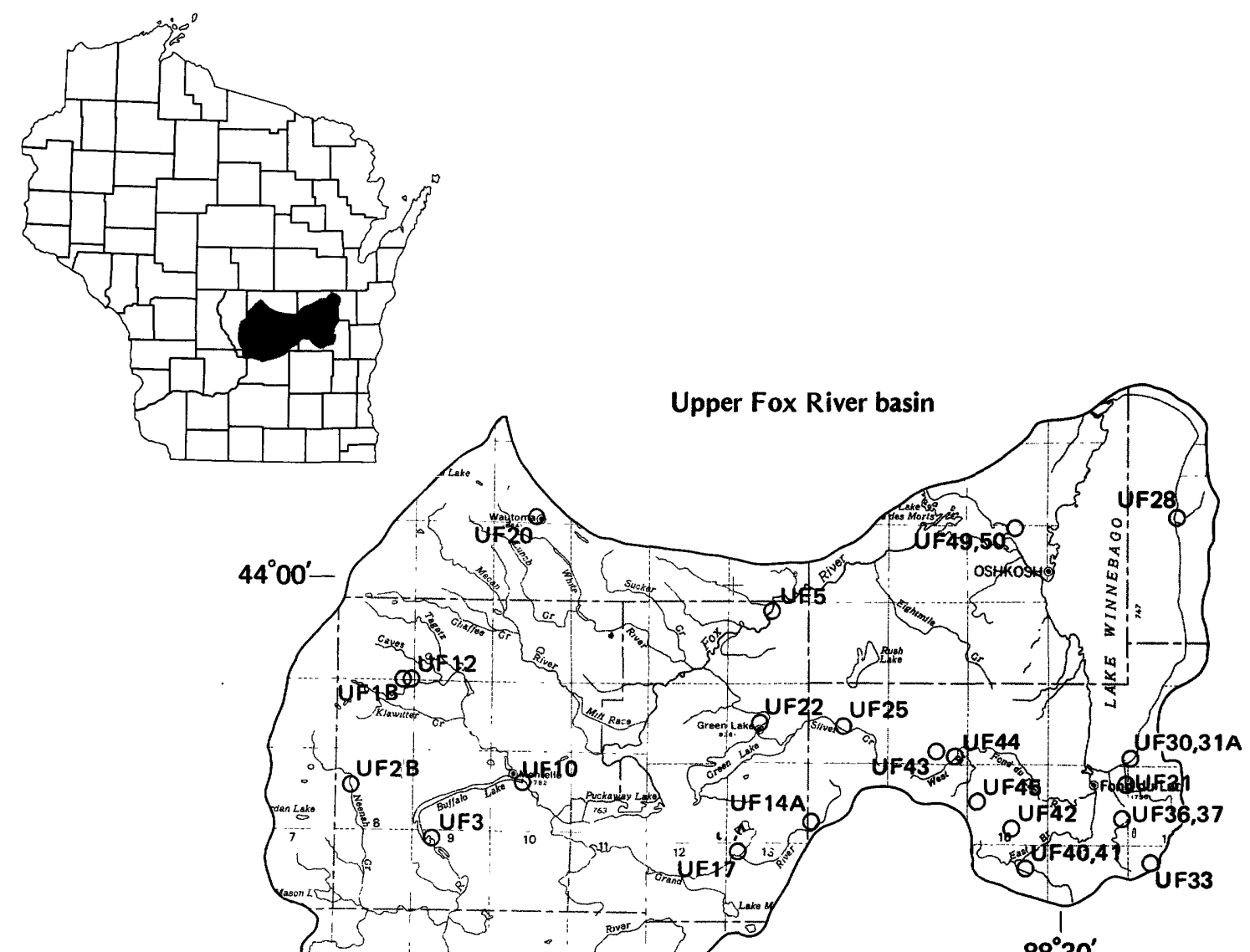

$88^{\circ} 30^{\prime}$

Upper Fox River basin

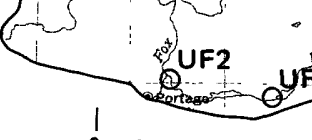

$89^{\circ} 30^{\prime}$

$89^{\circ} 00^{\prime}$

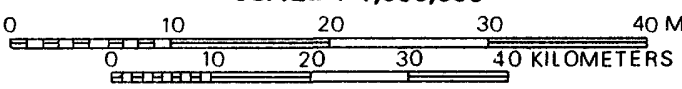

Base from U.S. Geological Survey

State base map, 1968

\begin{tabular}{|c|c|c|c|c|c|c|c|c|}
\hline $\begin{array}{l}\text { Station } \\
\text { number }\end{array}$ & 1 Stream & Station location & $\begin{array}{l}\text { Drainage } \\
\text { area } \\
\left(\mathbf{m i}^{2}\right)\end{array}$ & Date & $\begin{array}{c}\text { Discharge } \\
\left(\mathrm{ft}^{3} / \mathrm{s}\right)\end{array}$ & $\begin{array}{l}Q_{7,2} \\
\left(\mathrm{ft}^{3} / \mathrm{s}\right)(\end{array}$ & $\begin{array}{l}27,10 \\
3 / 5\}\end{array}$ & $\begin{array}{c}\text { Accuracy } \\
\text { level }\end{array}$ \\
\hline UF 1 & Fox River & $\begin{array}{l}\text { SW1/4NE } 1 / 4 \text { sec. } 4, \text { T. } 12 \text { N., R. } 10 \text { E., } \\
\text { Columbia County, at outlet of Spring } \\
\text { Lake, in Pardeeville, Wis. }\end{array}$ & 54.0 & $\begin{array}{l}\text { Nov. } 28,1972 \\
\text { July } 19,1973 \\
\text { Aug. } 22,1973 \\
\text { Sept. 23, } 1974 \\
\text { Oct. } 28,1975 \\
\text { June } 21,1976 \\
\text { Sept. 16, } 1976\end{array}$ & $\begin{array}{l}28.6 \\
51.5 \\
49.7 \\
29.3 \\
22.5 \\
6.19 \\
5.50\end{array}$ & 7.2 & 2.5 & c \\
\hline UF2 & Fox River & $\begin{array}{l}\text { NE } 1 / 4 N W 1 / 4 \text { sec. } 4, T .12 \text { N., R. } 9 \text { E., } \\
\text { Columbia County, at bridge on State } \\
\text { Highway } 33,1.7 \text { mi northeast of } \\
\text { Portage, Wis. }\end{array}$ & 72.3 & $\begin{array}{l}\text { Oct. } 23,1931 \\
\text { Aug. } 7,1972 \\
\text { Sept. } 11,1972 \\
\text { Nov. } 28,1972 \\
\text { July } 9,1973 \\
\text { Aug. } 20,1973 \\
\text { Sept. 23, } 1974 \\
\text { Oct. } 28,1975 \\
\text { June } 21,1976\end{array}$ & $\begin{array}{l}22.5 \\
24.1 \\
56.6 \\
53.0 \\
35.1 \\
33.6 \\
27.9 \\
20.6 \\
15.2\end{array}$ & 15 & 11 & c \\
\hline UF3 & Fox River & $\begin{array}{l}\text { SE } 1 / 4 \text { NW } 1 / 4 \text { sec. } 5, T .14 \text { N., R. } 9 \text { E., } \\
\text { Marquette County, at inlet to } \\
\text { Buffalo Lake, } 0.7 \text { mi northeast } \\
\text { of Endeavor, Wis. }\end{array}$ & 335 & $\begin{array}{l}\text { Sept. } 16,1976 \\
\text { Aug. } 30,1977 \\
\text { Aug. } 15,1978\end{array}$ & $\begin{array}{l}90.8 \\
118 \\
139\end{array}$ & 110 & 90 & c \\
\hline
\end{tabular}


Table 10. Upper Fox River basin station locations and low-flow-characteristics-Continued.

\begin{tabular}{|c|c|c|c|c|c|c|c|c|}
\hline $\begin{array}{l}\text { Station } \\
\text { number }\end{array}$ & 1 Stream & Station location & $\begin{array}{l}\text { Drainage } \\
\text { area } \\
\left(\mathrm{mi}^{2}\right)\end{array}$ & Date & $\begin{array}{c}\text { Discharge } \\
\left(\mathrm{ft}^{3} / \mathrm{s}\right)\end{array}$ & $\begin{array}{l}\mathrm{Q}_{7,2} \mathrm{Q}_{7} \\
\left(\mathrm{ft}^{3} / \mathrm{s}\right)\left(\mathrm{ft}^{3}\right.\end{array}$ & $t^{7 / 5} / \mathrm{s}^{3} \mathrm{~A}$ & $\begin{array}{c}\text { Accuracy } \\
\text { level }\end{array}$ \\
\hline UF5 & Fox River & $\begin{array}{l}\text { NW1/4NEY// sec. } 16, T .17 \mathrm{~N} ., \text { R. } 13 \text { E., } \\
\text { Green Lake County, } 0.4 \mathrm{mi} \\
\text { downstream from dam, } 1.0 \mathrm{mi} \text { south } \\
\text { of Berlin, Wis. }\end{array}$ & 1,430 & & & 4803 & 360 & $\mathbf{a}$ \\
\hline UF 10 & Fox River & $\begin{array}{l}\text { NW1/4NW1/4 sec. } 16, T .15 \text { N., R. } 10 \text { E., } \\
\text { Marquette County, at bridge on } \\
\text { State Highway 22, in Montello, Wis. }\end{array}$ & 392 & $\begin{array}{l}\text { Oct. } 19,1970 \\
\text { Aug. } 24,1976 \\
\text { Sept. } 16,1976 \\
\text { Aug. } 30,1977\end{array}$ & $\begin{array}{c}128 \\
138 \\
94.0 \\
131\end{array}$ & 120 & 92 & c \\
\hline UF 12 & $\begin{array}{l}\text { Westfield } \\
\text { Creek }\end{array}$ & $\begin{array}{l}\text { NEY/4NEY//4 sec. 12, T. } 16 \text { N., R. } 8 \text { E., } \\
\text { Marquette County, just upstream } \\
\text { from sewage-treatment plant, at } \\
\text { Westfield, Wis. }\end{array}$ & 23.4 & $\begin{array}{l}\text { Sept. } 11,1972 \\
\text { July } 9,1973 \\
\text { Aug. } 20,1973 \\
\text { Oct. 29, } 1975 \\
\text { June } 22,1976 \\
\text { Sept. 15, } 1976\end{array}$ & $\begin{array}{l}40.4 \\
50.6 \\
40.7 \\
44.1 \\
28.7 \\
32.2\end{array}$ & 27 & 23 & c \\
\hline UF 14A & Grand River & $\begin{array}{l}\text { SW1/4NW1/4 sec. 31, T. } 15 \mathrm{~N} ., \text { R. } 14 \mathrm{E} . \text {., } \\
\text { Fond du Lac County, just upstream } \\
\text { from sewage-disposal ponds, } 1.0 \mathrm{mi} \\
\text { southwest of Fairwater, Wis. }\end{array}$ & 24.7 & $\begin{array}{l}\text { July } 19,1973 \\
\text { Aug. } 22,1973 \\
\text { June } 24,1976 \\
\text { Sept. } 14,1976 \\
\text { July } 28,1977 \\
\text { Aug. } 29,1977\end{array}$ & $\begin{array}{l}7.96 \\
4.63 \\
2.33 \\
1.22 \\
1.87 \\
1.06\end{array}$ & 1.0 & .37 & c \\
\hline UF17 & Grand River & $\begin{array}{l}\text { NE } 1 / 4 N E 1 / 4 \text { sec. } 7, T .14 \text { N., R. } 13 \text { E., } \\
\text { Green Lake County, at bridge on } \\
\text { State Highway } 44 \text {, at Markesan, Wis. }\end{array}$ & 57.6 & $\begin{array}{l}\text { July } 19,1973 \\
\text { Sept. } 24,1974 \\
\text { Oct. } 25,1975 \\
\text { June } 24,1976 \\
\text { Sept. } 14,1976\end{array}$ & $\begin{array}{l}21.2 \\
8.55 \\
5.01 \\
12.5 \\
4.66\end{array}$ & 5.5 & 2.3 & c \\
\hline UF20 & White River & $\begin{array}{l}\text { SE } 1 / 4 N E 1 / 4 \text { sec. } 3, T .18 \mathrm{~N} ., \text { R. } 10 \text { E., } \\
\text { Waushara County, just upstream from } \\
\text { sewage-treatment plant, at Wautoma, } \\
\text { Wis. }\end{array}$ & 16.5 & $\begin{array}{l}\text { Sept. } 11,1972 \\
\text { July 17, } 1973 \\
\text { Aug. } 20,1973 \\
\text { Sept. 24, } 1974 \\
\text { Oct. 29, } 1975 \\
\text { June 22, } 1976 \\
\text { Sept. 15, } 1976\end{array}$ & $\begin{array}{l}18.7 \\
19.9 \\
20.5 \\
19.6 \\
19.5 \\
16.3 \\
14.1\end{array}$ & 15 & 14 & c \\
\hline UF22 & Puchyan River & $\begin{array}{l}\text { NE } 1 / 4 \text { SE } 1 / 4 \text { sec. } 16, T .16 \mathrm{~N} ., \text { R. } 13 \text { E., } \\
\text { Green Lake County, at bridge on } \\
\text { State Highways } 23 \text { and } 49,1.1 \mathrm{mi} \\
\text { northeast of Green Lake, Wis. }\end{array}$ & 117 & $\begin{array}{l}\text { Nov. } 29,1972 \\
\text { Oct. } 29,1975 \\
\text { June } 23,1976 \\
\text { Sept. } 14,1976 \\
\text { Aug. } 29,1977\end{array}$ & $\begin{array}{l}68.7 \\
10.4 \\
21.0 \\
6.82 \\
8.24\end{array}$ & $\underline{6}$ & $\underline{6}$ & \\
\hline UF25 & Silver Creek & $\begin{array}{l}\text { SW } 1 / 4 \text { SE } 1 / 4 \text { sec. } 17, T .16 \mathrm{~N} ., \text { R. } 14 \text { E., } \\
\text { Fond du Lac County, at Ripon } \\
\text { sewage-treatment plant, } 1.1 \mathrm{mi} \\
\text { northwest of Ripon, Wis. }\end{array}$ & 26.7 & $\begin{array}{l}\text { July } 17,1973 \\
\text { Aug. } 20,1973 \\
\text { Sept. 25, } 1974 \\
\text { Oct. } 29,1975 \\
\text { June } 23,1976 \\
\text { Sept. 14, } 1976\end{array}$ & $\begin{array}{l}9.85 \\
7.38 \\
5.58 \\
3.49 \\
4.85 \\
3.23\end{array}$ & 3.3 & 1.7 & c \\
\hline UF28 & Mud Creek & $\begin{array}{l}\text { Lat } 44^{\circ} 03^{\prime} 12^{\prime \prime} \text {, long } 88^{\circ} 19^{\prime} 03^{\prime \prime}, \text {, Calumet } \\
\text { County, at bridge on country road, } \\
2.7 \text { mi north of Quinney, } 1.6 \mathrm{mi} \\
\text { southwest of Stockbridge, Wis. }\end{array}$ & t & $\begin{array}{l}\text { July } 18,1973 \\
\text { July } 31,1973 \\
\text { Sept. } 4,1974 \\
\text { July } 13,1977\end{array}$ & $\begin{array}{l}2 \\
2 \\
4 \\
{ }_{0}^{0} \\
{ }_{0}^{0}\end{array}$ & 0 & 0 & c \\
\hline $\begin{array}{l}\text { UF30, } \\
31 A\end{array}$ & $\begin{array}{l}\text { Taycheedah } \\
\text { Creek } \\
\text { tributary }\end{array}$ & $\begin{array}{l}\text { NW1/4NW1/4 sec. } 6, T .15 \text { N., R. } 18 \text { E., } \\
\text { Fond du Lac County, at bridge on } \\
\text { County Trunk K, } 0.3 \text { mi south of } \\
\text { Taycheedah, Wis. }\end{array}$ & 0.54 & $\begin{array}{l}\text { Sept. } 12,1972 \\
\text { July } 18,1973 \\
\text { Aug. } 21,1973 \\
\text { Sept. 24, } 1974 \\
\text { Oct. 29, } 1975 \\
\text { June } 23,1976\end{array}$ & $\begin{array}{l}2 \\
4 \\
4_{0}^{0} \\
0 \\
4_{0}^{01} \text { est. } \\
4_{0}^{0}\end{array}$ & . & 0 & c \\
\hline UF31 & $\begin{array}{l}\text { Taycheedah } \\
\text { Creek }\end{array}$ & $\begin{array}{l}\text { SW } 1 / 4 N E 1 / 4 \text { sec. } 12, T .15 \mathrm{~N} ., \text { R. } 17 \text { E., } \\
\text { Fond du Lac County, at bridge on } \\
\text { State Highway } 23,2.0 \text { mi east of } \\
\text { Fond du Lac, Wis. }\end{array}$ & 14.4 & $\begin{array}{l}\text { Nov. } 30,1972 \\
\text { July } 18,1973 \\
\text { Aug. } 21,1973 \\
\text { Sept. } 24,1974 \\
\text { Oct. } 30,1975 \\
\text { June } 23,1976 \\
\text { Sept. } 14,1976\end{array}$ & $\begin{array}{l}4.52 \\
1.35 \\
2.13 \\
.52 \\
.63 \\
.65 \\
2.0\end{array}$ & .06 & $3<.01$ & c \\
\hline
\end{tabular}


Table 10. Upper Fox River basin station locations and low-flow-characteristics-Continued.

\begin{tabular}{|c|c|c|c|c|c|c|c|c|}
\hline $\begin{array}{l}\text { Station } \\
\text { number }\end{array}$ & $n^{1} \underset{\text { name }}{\text { Stream }}$ & Station location & $\begin{array}{c}\text { Drainage } \\
\text { area } \\
\left(\mathrm{mi}^{2}\right)\end{array}$ & Date & $\begin{array}{c}\text { Discharge } \\
\left(\mathrm{ft}^{3} / \mathrm{s}\right)\end{array}$ & $\begin{array}{l}\mathrm{O}_{7,2} \mathrm{O}_{7} \\
\left(\mathrm{ft}^{3} / \mathrm{s}\right)\left(\mathrm{ft}^{2}\right.\end{array}$ & $\begin{array}{l}7,10 \\
3 / s)\end{array}$ & $\begin{array}{c}\text { Accuracy } \\
\text { level }\end{array}$ \\
\hline UF33 & $\begin{array}{l}\text { De Neveu Creek } \\
\text { tributary }\end{array}$ & $\begin{array}{l}\text { SE1/4NE1/4 sec. } 8, T .14 \text { N., R. } 18 \text { E., } \\
\text { Fond du Lac County, just upstream } \\
\text { from sewage-treatment plant, } 0.3 \mathrm{mi} \\
\text { north of Eden, Wis. }\end{array}$ & 2.85 & $\begin{array}{l}\text { Nov. } 15,1971 \\
\text { July } 18,1973 \\
\text { Aug. } 21,1973 \\
\text { Sept. 25, } 1974 \\
\text { Oct. } 30,1975 \\
\text { Sept. } 13,1976\end{array}$ & $\begin{array}{l}0.34 \\
.44 \\
.61 \\
.30 \\
.26 \\
.20\end{array}$ & 0.12 & 0.04 & c \\
\hline $\begin{array}{l}\text { UF36, } \\
37\end{array}$ & De Neveu Creek & $\begin{array}{l}\text { NE1/4NE1/4 sec. } 25, T .15 \text { N., R. } 17 \text { E., } \\
\text { Fond du Lac County, at site on U.S. } \\
\text { Highway } 45,3.1 \text { mi southeast of } \\
\text { post office in Fond du Lac, Wis. }\end{array}$ & 16.4 & $\begin{array}{l}\text { Nov. } 16,1971 \\
\text { July } 18,1973 \\
\text { Aug. } 21,1973 \\
\text { Oct. } 30,1975 \\
\text { June } 23,1976 \\
\text { Sept. } 14,1976\end{array}$ & $\begin{array}{l}1.18 \\
2.00 \\
2.96 \\
1.27 \\
1.32 \\
.75\end{array}$ & .77 & .39 & c \\
\hline UF40, & $\begin{array}{l}\text { Campground } \\
\text { Creek }\end{array}$ & $\begin{array}{l}\text { SE } 1 / 4 \text { SW } 1 / 4 \text { sec. } 11, T .14 \text { N., R. } 16 \text { E., } \\
\text { Fond du Lac County, just upstream } \\
\text { from sewage-treatment plant, at } \\
\text { Oakfield, Wis. }\end{array}$ & 9.44 & $\begin{array}{l}\text { Nov. } 16,1971 \\
\text { July } 18,1973 \\
\text { Aug. } 21,1973 \\
\text { Sept. } 24,1974 \\
\text { Oct. } 30,1975 \\
\text { June } 24,1976 \\
\text { Sept. } 13,1976 \\
\text { July } 13,1976\end{array}$ & $\begin{array}{l}2.61 \\
6.57 \\
5.76 \\
4.67 \\
2.98 \\
4.39 \\
3.21 \\
3.48\end{array}$ & 2.9 & 1.8 & c \\
\hline UF42 S & $\begin{array}{l}\text { Sevenmile } \\
\text { Creek }\end{array}$ & $\begin{array}{l}\text { NW1/4NE1/4 sec. } 34, \text { T. } 15 \text { N., R. } 16 \text { E., } \\
\text { Fond du Lac County, at bridge on } \\
\text { State Highway 103, at Lamartine, } \\
\text { Wis. }\end{array}$ & 17.5 & $\begin{array}{l}\text { July } 18,1973 \\
\text { Aug. } 22,1973 \\
\text { Sapt. 24, } 1974 \\
\text { June 24, } 1976\end{array}$ & $\begin{array}{l}<.01 \text { est. } \\
2_{0}^{.01} \text { est. } \\
2_{0}^{0}\end{array}$ & . & 0 & c \\
\hline UF43 & $\begin{array}{l}\text { West Branch } \\
\text { Fond du Lac } \\
\text { River tributary }\end{array}$ & $\begin{array}{l}\text { NE1/1/4W1/4 sec. } 35, T .16 \text { N., R. } 15 \text { E., } \\
\text { Fond du Lac County, at bridge on } \\
\text { State Highway } 26,0.8 \text { mi north } \\
\text { of Rosendale, Wis. }\end{array}$ & 2.85 & $\begin{array}{l}\text { Nov. } 16,1971 \\
\text { Nov. } 15,1973 \\
\text { Sept. } 24,1974 \\
\text { Oct. } 29,1975 \\
\text { Sept. } 13,1976 \\
\text { July } 28,1977 \\
\text { Aug. } 29,1977\end{array}$ & $\begin{array}{l}.66 \\
4.63 \\
0 \\
4.02 \\
.16 \\
.65\end{array}$ & .02 & $<.01$ & c \\
\hline UF44 V & $\begin{array}{l}\text { West Branch } \\
\text { Fond du Lac } \\
\text { River }\end{array}$ & $\begin{array}{l}\text { NW } 1 / 4 \text { SW } 1 / 4 \text { sec. } 36, T .16 \text { N., R. } 15 \text { E., } \\
\text { Fond du Lac County, at culverts } \\
\text { on County Trunk OOO, at sewage } \\
\text { treatment plant, } 0.9 \text { mi northeast of } \\
\text { Rosendale, Wis. }\end{array}$ & 5.32 & $\begin{array}{l}\text { Nov. } 16,1971 \\
\text { July } 17,1973 \\
\text { Aug. } 20,1973 \\
\text { Nov. } 14,1973 \\
\text { Oct. } 29,1975 \\
\text { June } 24,1976 \\
\text { July } 13,1976 \\
\text { Sept. } 13,1976\end{array}$ & $\begin{array}{l}.97 \\
.65 \\
.14 \\
.40 \\
.26 \\
.56 \\
.17 \\
.0\end{array}$ & .03 & 0 & c \\
\hline $\begin{array}{ll}\text { UF45 } & s \\
t\end{array}$ & $\begin{array}{l}\text { Sevenmile Creek } \\
\text { tributary }\end{array}$ & $\begin{array}{l}\text { NEY/4SW1/4 sec. 19, T. } 15 \text { N., R. } 16 \text { E., } \\
\text { Fond du Lac County, at bridge on } \\
\text { County Trunk C, } 9.4 \text { mi west of } \\
\text { post office in Fond du Lac, Wis. }\end{array}$ & 1.29 & $\begin{array}{l}\text { Nov. } 17,1971 \\
\text { Sept. } 24,1974 \\
\text { Oct. } 29,1975 \\
\text { June } 24,1976 \\
\text { Sept. } 13,1976 \\
\text { July } 28,1977 \\
\text { Aug. } 29,1977\end{array}$ & $\begin{array}{l}4.66 \\
.22 \\
.32 \\
.18 \\
.17 \\
.12\end{array}$ & .09 & $<.01$ & c \\
\hline $\begin{array}{l}\text { UF49, } \\
50\end{array}$ & $\begin{array}{l}\text { Fox River } \\
\text { tributary }\end{array}$ & $\begin{array}{l}\text { SE } 1 / 4 N W 1 / 4 \text { sec. } 3, T .18 \text { N., R. } 16 \text { E., } \\
\text { Winnebago County, at bridge on } \\
\text { State Highway } 110,3.7 \text { mi northwest } \\
\text { of city hall, in Oshkosh, Wis. }\end{array}$ & .68 & $\begin{array}{l}\text { July } 17,1973 \\
\text { Aug. } 21,1973 \\
\text { Sept. } 24,1974 \\
\text { Oct. } 29,1975 \\
\text { June } 23,1976\end{array}$ & $\begin{array}{l}{ }_{4}^{0} \\
4_{0}^{0} \\
4_{0}^{0} \\
4_{0}^{0}\end{array}$ & 0 & 0 & c \\
\hline UF1B $\begin{array}{ll}v \\
t\end{array}$ & $\begin{array}{l}\text { Westfield Creek } \\
\text { tributary }\end{array}$ & $\begin{array}{l}\text { SEY/4SW1/4 sec. 1, T. } 16 \text { N., R. } 8 \text { E., } \\
\text { Marquette County, just upstream } \\
\text { from fish hatchery outfall, at } \\
\text { Westfield, Wis. }\end{array}$ & 1.02 & $\begin{array}{l}\text { June } 22,1976 \\
\text { Sept. } 15,1976 \\
\text { July } 29,1977 \\
\text { Aug. } 30,1977\end{array}$ & $\begin{array}{l}4.05 \\
.05 \\
.02\end{array}$ & .02 & $<.01$ & c \\
\hline
\end{tabular}


Table 10. Upper Fox River basin station locations and low-flow-characteristics-Continued.

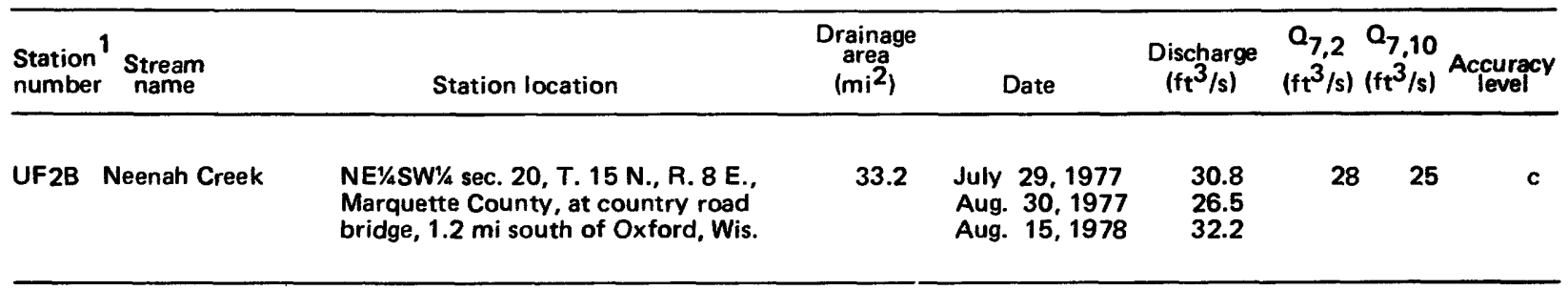

1Based on 1967 report by Wisconsin Department of Naturel Resources (DNR). Site lettered "B" was not included in 1967 DNR report.

2 Streambed is dry.

${ }^{4}$ Negligible discharge--water ponded, unable to measure velocity.

6 No estimate possible due to regulation upstream. 
Table 11. Grant-Platte River basin station locations and low-flow characteristics.

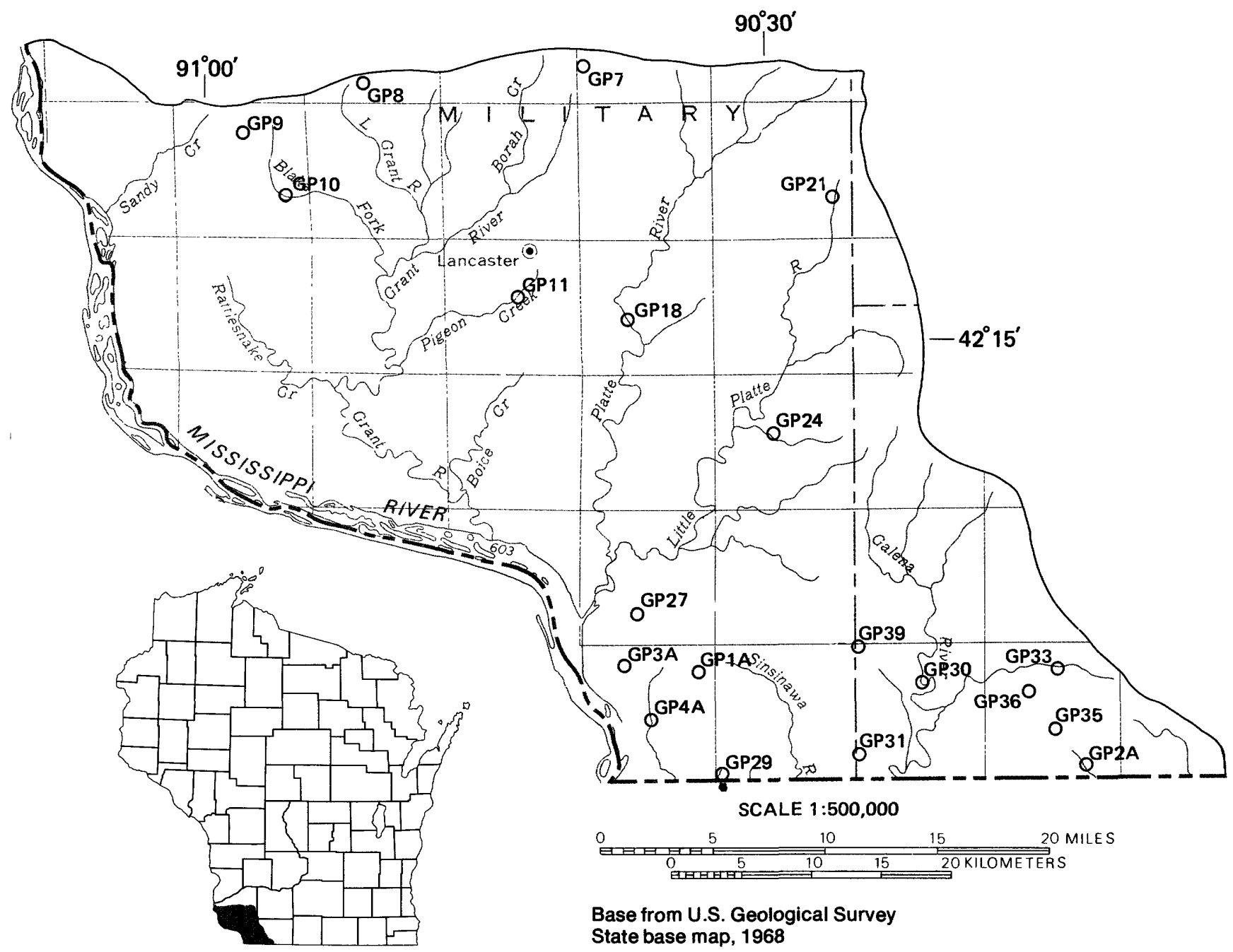

Grant-Platte River basin

\begin{tabular}{|c|c|c|c|c|c|c|c|c|}
\hline $\begin{array}{l}\text { Station } \\
\text { number }\end{array}$ & Stream & Station location & $\begin{array}{l}\text { Drainage } \\
\text { area } \\
\left(\mathrm{mi}^{2}\right)\end{array}$ & Date & $\begin{array}{c}\text { Discharge } \\
\left(\mathrm{ft}^{3} / \mathrm{s}\right)\end{array}$ & $\mathrm{Q}_{7,2} \mathrm{Q}_{7}$ & $\begin{array}{l}70 \\
3 / 5)\end{array}$ & $\underset{\text { Accuracy }}{\text { Aevel }}$ \\
\hline GP7 & Gregory Branch & $\begin{array}{l}\text { SE } 1 / 4 \text { SW } 1 / 4 \text { sec. } 19, \text { T. } 6 \text { N., R. } 2 \text { W., } \\
\text { Grant County, at sewage-treatment } \\
\text { plant, in Fennimore, Wis. }\end{array}$ & 0.42 & $\begin{array}{lc}\text { June } & 21,1972 \\
\text { Sept. } 1,1972 \\
\text { Aug. } 3,1973 \\
\text { Oct. } 17,1975 \\
\text { July } 27,1976 \\
\text { Oct. } 26,1976\end{array}$ & $\begin{array}{l}0.14 \\
.28 \\
.24 \\
.12 \\
.05 \\
.05\end{array}$ & 0.02 & 0.01 & c \\
\hline GP8 & $\begin{array}{l}\text { Little Grant } \\
\text { River }\end{array}$ & $\begin{array}{l}\text { NE } 1 / 4 \text { SW } 1 / 4 \text { sec. } 28, \text { T. } 6 \text { N., R. } 4 \text { W., } \\
\text { Grant County, at sewage-disposal } \\
\text { reservoir, in Mount Hope, Wis. }\end{array}$ & .56 & $\begin{array}{l}\text { June } 21,1972 \\
\text { Sept. } 1,1972 \\
\text { Aug. } 3,1973 \\
\text { Oct. } 17,1975 \\
\text { July } 27,1976 \\
\text { Oct. } 26,1976\end{array}$ & $\begin{array}{l}.09 \\
.13 \\
.39 \\
.18 \\
.10 \\
.03\end{array}$ & .01 & $<.01$ & c \\
\hline
\end{tabular}


Table 11. Grant-Platte River basin station locations and low-flow characteristics-Continued.

\begin{tabular}{|c|c|c|c|c|c|c|c|c|}
\hline $\begin{array}{l}\text { Station } \\
\text { number }\end{array}$ & 1 Stream & Station locetion & $\begin{array}{l}\text { Drainage } \\
\text { area } \\
\left(\mathrm{mi}^{2}\right)\end{array}$ & Date & $\begin{array}{c}\text { Discharge } \\
\left(\mathrm{ft}^{3} / \mathrm{s}\right)\end{array}$ & $\begin{array}{l}\mathrm{Q}_{7,2} \mathrm{Q}_{7} \\
\left(\mathrm{ft}^{3} / \mathrm{s}\right)\left(\mathrm{ft}^{3}\right.\end{array}$ & 3,10 & $\begin{array}{c}\text { Accuracy } \\
\text { level }\end{array}$ \\
\hline GP9 & $\begin{array}{l}\text { Blake Fork } \\
\text { tributary }\end{array}$ & $\begin{array}{l}\text { SW1/4SE } 1 / 4 \text { sec. } 4, T .5 \text { N., R. } 5 \text { W., } \\
\text { Grant County, at sewage-disposai } \\
\text { site, } 0.5 \text { mi south of Patch } \\
\text { Grove, Wis. }\end{array}$ & 1.31 & $\begin{array}{l}\text { June } 21,1972 \\
\text { Aug. } 31,1972 \\
\text { Aug. } 3,1973 \\
\text { Oct. } 17,1975 \\
\text { July } 27,1976 \\
\text { Oct. } 26,1976\end{array}$ & $\begin{array}{l}0_{4} 04 \\
.49 \\
.15 \\
.05 \\
.03\end{array}$ & 0.01 & $<0.01$ & c \\
\hline GP10 & Blake Fork & $\begin{array}{l}\text { SE1/4NE1// sec. 26, T. } 5 \text { N., R. } 5 \text { W., } \\
\text { Grant County, at sewage-disposal } \\
\text { site, in Bloomington, Wis. }\end{array}$ & 17.9 & $\begin{array}{l}\text { June } 21,1972 \\
\text { Aug. } 31,1972 \\
\text { Aug. } 3,1973 \\
\text { Oct. } 17,1975 \\
\text { July } 27,1976 \\
\text { Oct. } 26,1976\end{array}$ & \begin{tabular}{c|}
3.88 \\
8.30 \\
12.8 \\
7.04 \\
4.32 \\
4.29
\end{tabular} & 2.5 & 1.5 & c \\
\hline \multirow[t]{2}{*}{ GP11 } & $\begin{array}{l}\text { Pigeon Creek } \\
\text { tributary }\end{array}$ & $\begin{array}{l}\text { SW } 1 / 4 N E 1 / 4 \text { sec. } 10, \text { T. } 4 \text { N., R. } 3 \text { W., } \\
\text { Grent County, at bridge on town } \\
\text { road to Lancaster sewage-treatment } \\
\text { plant. }\end{array}$ & .31 & June 21, 1972 & ${ }^{2} 0$ & 0 & 0 & c \\
\hline & Pigeon Creek & $\begin{array}{l}\text { SWY/aSW1/a sec. 15, T. } 4 \text { N., R. } 3 \text { W., } \\
\text { Grant County, at town road, } 2.3 \mathrm{mi} \\
\text { south of Lancaster, Wis. }\end{array}$ & 6.93 & $\begin{array}{l}\text { June } 21,1972 \\
\text { Aug. } 31,1972 \\
\text { Aug. } 2,1973 \\
\text { Oct. } 17,1975 \\
\text { July } 26,1976 \\
\text { Oct. } 26,1976\end{array}$ & $\begin{array}{l}32.66 \\
4.71 \\
4.91 \\
3.01 \\
4.10 \\
1.55\end{array}$ & 1.5 & .85 & b \\
\hline \multirow[t]{2}{*}{ GP18 } & $\begin{array}{l}\text { Austin Brench } \\
\text { tributary }\end{array}$ & $\begin{array}{l}\text { SW } 1 / \text { NWW } 1 / 4 \text { sec. } 13, T .4 \text { N., R. } 3 \text { W., } \\
\text { Grant County, at County Hospital } \\
\text { sewage-treatment plent, } 2.1 \text { mi } \\
\text { southeast of Lancaster, Wis. }\end{array}$ & .03 & & & & & \\
\hline & Austin Branch & $\begin{array}{l}\text { NW1/4SE } 1 / 4 \text { sec. } 17, \text { T. } 4 \text { N., R. } 2 \text { W., } \\
\text { Grant County, at mouth, } 2.7 \text { mi } \\
\text { northwest of Ellenboro, Wis. }\end{array}$ & 5.52 & $\begin{array}{l}\text { Sept. } 18,1969 \\
\text { Aug. } 18,1970 \\
\text { Sept. } 1,1971 \\
\text { June } 21,1972 \\
\text { June } 27,1973 \\
\text { Aug. } 2,1973 \\
\text { Oct. } 17,1975 \\
\text { July } 26,1976\end{array}$ & $\begin{array}{l}3.40 \\
3.43 \\
3.12 \\
3.00 \\
8.94 \\
5.74 \\
3.21 \\
2.87\end{array}$ & 1.6 & 1.0 & c \\
\hline \multirow[t]{2}{*}{ GP21 } & $\begin{array}{l}\text { Little Platte } \\
\text { River }\end{array}$ & $\begin{array}{l}\text { SWY/4NEY// sec. } 24, \text { T. } 5 \text { N., R. } 1 \text { W., } \\
\text { Grant County, at sewage-treatment } \\
\text { plant, in Livingston, Wis. }\end{array}$ & .23 & & & & & \\
\hline & $\begin{array}{l}\text { Little Platte } \\
\text { River }\end{array}$ & $\begin{array}{l}\text { SW1/4SE } 1 / 4 \text { sec. } 24, \text { T. } 5 \text { N., R. } 1 \text { W., } \\
\text { Grant County, et bridge on Stete } \\
\text { Highway } 80 \text {, } 1.0 \text { mi south of } \\
\text { Livingston, Wis. }\end{array}$ & 1.29 & $\begin{array}{l}\text { June } 23,1972 \\
\text { Sept. } 1,1972 \\
\text { Aug. } 3,1973 \\
\text { Oct. } 17,1975 \\
\text { July } 26,1976 \\
\text { Oct. } 26,1976\end{array}$ & $\begin{array}{l}.26 \\
1.21 \\
1.13 \\
.40 \\
.17 \\
.17\end{array}$ & .09 & .03 & c \\
\hline GP24 & $\begin{array}{l}\text { Roundtree } \\
\text { Branch }\end{array}$ & $\begin{array}{l}\text { SE } 1 / 4 N E 1 / / 4 \text { sec. } 17, \text { T. } 3 \text { N., R. } 1 \text { W., } \\
\text { Grant County, et sewage-treatment } \\
\text { plant, in Pletteville, Wis. }\end{array}$ & 12.5 & $\begin{array}{lr}\text { Aug. } & 30,1972 \\
\text { June } 28,1973 \\
\text { Aug. } 2,1973 \\
\text { Oct. } 16,1975 \\
\text { July } 27,1976 \\
\text { Oct. } 27,1976\end{array}$ & $\begin{array}{c}4.75 \\
12.4 \\
8.94 \\
4.64 \\
6.83 \\
2.60\end{array}$ & 1.7 & .90 & c \\
\hline \multirow[t]{2}{*}{ GP27 } & $\begin{array}{l}\text { Indian Creek } \\
\text { tributary }\end{array}$ & $\begin{array}{l}\text { SW } 1 / \text { SW } 1 / \text { sec. } 22, \text { T. } 2 \text { N., R. } 2 \text { W., } \\
\text { Grant County, et sewage-treatment } \\
\text { plant, at Dickeyville, Wis. }\end{array}$ & .06 & & & & & \\
\hline & Indian Creek & $\begin{array}{l}\text { SW1/4NEY// sec. } 29, \text { T. } 2 \text { N., R: } 2 \text { W., } \\
\text { Grant County, at bridge on town } \\
\text { road, } 2.1 \text { mi west of Dickeyville, } \\
\text { Wis. }\end{array}$ & 3.72 & $\begin{array}{l}\text { June } 22,1972 \\
\text { Aug. } 30,1972 \\
\text { Aug. } 2,1973 \\
\text { Oct. } 16,1975 \\
\text { July } 27,1976 \\
\text { Oct. } 27,1976\end{array}$ & $\begin{array}{l}.05 \\
.01 \text { est. } \\
1.79 \\
.94 \\
.33 \\
.35\end{array}$ & $<.01$ & $<.01$ & c \\
\hline
\end{tabular}


Table 11. Grant-Platte River basin station locations and low-flow characteristics-Continued.

\begin{tabular}{|c|c|c|c|c|c|c|c|c|}
\hline $\begin{array}{l}\text { Station } \\
\text { number }\end{array}$ & Stream & Station location & $\begin{array}{l}\text { Drainage } \\
\text { area } \\
\left(\mathrm{mi}^{2}\right)\end{array}$ & Date & $\begin{array}{l}\text { Discharge } \\
\left(\mathrm{ft}^{3} / \mathrm{s}\right)\end{array}$ & $\begin{array}{l}a_{7,2} a_{7} \\
\left(\mathrm{ft}^{3} / \mathrm{s}\right)\left(\mathrm{ft}^{3}\right)\end{array}$ & $10 \mathrm{Ac}$ & $\begin{array}{c}\text { Iccuracy } \\
\text { level }\end{array}$ \\
\hline \multirow[t]{2}{*}{ GP29 } & $\begin{array}{l}\text { Menominee River } \\
\text { tributary }\end{array}$ & $\begin{array}{l}\text { NW1/4NW1/4 sec. } 31, \text { T. } 1 \text { N., R. } 1 \text { W., } \\
\text { Grant County, sewage-treatment } \\
\text { plant, at Sinsiniwa, Wis. }\end{array}$ & 0.04 & & & & & \\
\hline & $\begin{array}{l}\text { Menominee River } \\
\text { tributary }\end{array}$ & $\begin{array}{l}\text { SWY NWY// sec. } 31, \mathrm{~T} .1 \mathrm{~N} ., \text { R. } 1 \mathrm{~W} ., \\
\text { Grant County, at bridge on County } \\
\text { Trunk ZZ, } 0.5 \text { mi south of } \\
\text { Sinsiniwa, Wis. }\end{array}$ & .18 & $\begin{array}{l}\text { June } 22,1972 \\
\text { Aug. } 30,1972 \\
\text { Aug. } 1,1973 \\
\text { Oct. } 16,1975 \\
\text { Oct. } 27,1976\end{array}$ & $\begin{array}{r}0.08 \\
.09 \\
.14 \\
.07 \\
.02\end{array}$ & \multicolumn{3}{|c|}{$0.01<0.01$} \\
\hline \multirow[t]{2}{*}{ GP30 } & Galena River & $\begin{array}{l}\text { SW } 1 / 4 \text { SE } 1 / 4 \text { sec. } 10, \text { T. } 1 \text { N., R. } 1 \text { E., } \\
\text { Lafayette County, at bridge on } \\
\text { State Highway } 11,1.4 \text { mi east of } \\
\text { east of Benton, Wis. }\end{array}$ & 71.0 & $\begin{array}{l}\text { June } 22,1972 \\
\text { Aug. } 31,1972 \\
\text { Aug. } 1,1973 . \\
\text { Oct. } 15,1975 \\
\text { Oct. } 27,1976\end{array}$ & $\begin{array}{l}17.0 \\
27.3 \\
51.5 \\
23.5 \\
10.1\end{array}$ & 11 & 5.7 & c \\
\hline & Galena River & $\begin{array}{l}\text { NW1/4NE1/4 sec. } 16, \text { T. } 1 \text { N., R. } 1 \text { E., } \\
\text { Lafayette County, at sewage- } \\
\text { treatment plant outfall, at Benton, } \\
\text { Wis. }\end{array}$ & 71.6 & & & & & \\
\hline \multirow[t]{2}{*}{ GP31 } & $\begin{array}{l}\text { Scrabble Branch } \\
\text { tributary }\end{array}$ & $\begin{array}{l}\text { SE } 1 / 4 N E 1 / 4 \text { sec. } 25, \text { T. } 1 \text { N., R. } 1 \text { W., } \\
\text { Grant County, at sewage-treatment } \\
\text { plant, at Hazel Green, Wis. }\end{array}$ & .07 & & & & & \\
\hline & Scrabble Branch & $\begin{array}{l}\text { SW } 1 / 4 \text { NW1/4 sec. } 32, \text { T. } 1 \text { N., R. } 1 \text { E., } \\
\text { Lafayette County, at bridge on } \\
\text { town road, } 2.1 \text { mi southeast of } \\
\text { Hazel Green, Wis. }\end{array}$ & 4.69 & $\begin{array}{l}\text { June } 22,1972 \\
\text { Aug. } 30,1972 \\
\text { Aug. } 1,1973 \\
\text { Oct. } 16,1975 \\
\text { Oct. } 27,1976\end{array}$ & $\begin{array}{l}1.03 \\
1.07 \\
2.79 \\
1.39 \\
.67\end{array}$ & .66 & .37 & c \\
\hline GP33 & $\begin{array}{l}\text { Shullsburg } \\
\text { Branch }\end{array}$ & $\begin{array}{l}\text { NE } 1 / 4 \text { NW1/4 sec. } 10, \text { T. } 1 \text { N., R. } 2 \text { E., } \\
\text { Lafayette County, at bridge on } \\
\text { County Trunk O, at Shullsburg, } \\
\text { Wis. }\end{array}$ & 7.72 & $\begin{array}{l}\text { June } 22,1972 \\
\text { Aug. } 31,1972 \\
\text { Aug. } 1,1973 \\
\text { Oct. } 15,1975 \\
\text { Oct. } 27,1976\end{array}$ & $\begin{array}{l}1.10 \\
2.69 \\
4.91 \\
1.57 \\
.35\end{array}$ & .33 & .09 & c \\
\hline GP35 & $\begin{array}{l}\text { Shullsburg } \\
\text { Branch tributary }\end{array}$ & $\begin{array}{l}\text { NW1/4SW/4 sec. } 22, \text { T. } 1 \text { N., R. } 2 \text { E., } \\
\text { Lafayette County, at culvert on } \\
\text { County Trunk O, } 2.2 \text { mi southwest } \\
\text { of Shullsburg, Wis. }\end{array}$ & .32 & $\begin{array}{l}\text { Oct. } 15,1975 \\
\text { Oct. } 27,1976 \\
\text { June } 22,1977\end{array}$ & $\begin{array}{l}4.78 \\
3.59 \\
2.76\end{array}$ & $\underline{5}$ & 5 & \\
\hline GP36 & $\begin{array}{l}\text { Shullsburg } \\
\text { Branch tributary }\end{array}$ & $\begin{array}{l}\text { NE } 1 / 4 \text { NE1/4 sec. } 17, T \text {. } 1 \text { N., R. } 2 \text { E., } \\
\text { Lafayette County, at bridge on } \\
\text { State Highway } 11,2.2 \text { mi west of } \\
\text { Shullsburg, Wis. }\end{array}$ & 3.94 & $\begin{array}{l}\text { Oct. } 15,1975 \\
\text { Oct. 27, } 1976 \\
\text { June 22, } 1977\end{array}$ & $\begin{array}{l}3.57 \\
2.36 \\
2.07\end{array}$ & $\underline{5}$ & $\underline{5}$ & \\
\hline \multirow[t]{2}{*}{ GP39 } & $\begin{array}{l}\text { Coon Branch } \\
\text { tributary }\end{array}$ & $\begin{array}{l}\text { NW1/4SW1/4 sec. } 31, \text { T. } 2 \text { N., R. } 1 \text { E., } \\
\text { Lafayette County, at sewage- } \\
\text { treatment plant, at Cuba City, Wis. }\end{array}$ & .09 & & & & & \\
\hline & Coon Branch & $\begin{array}{l}\text { SW } / 4 \text { SE } / 4 \text { sec. } 31, \text { T. } 2 \text { N., R. } 1 \text { E., } \\
\text { Lafayette County, at bridge on } \\
\text { town road, } 1.4 \text { mi southeast of } \\
\text { Cuba City, Wis. }\end{array}$ & 1.01 & $\begin{array}{l}\text { June } 22,1972 \\
\text { Aug. } 30,1972 \\
\text { Aug. } 2,1973 \\
\text { Oct. } 16,1975 \\
\text { Oct. } 27,1976\end{array}$ & $\begin{array}{l}.28 \\
.35 \\
.56 \\
.25 \\
.18\end{array}$ & .17 & .12 & c \\
\hline GP1A & Louisburg Creek & $\begin{array}{l}\text { NE } 1 / 4 N W 1 / 4 \text { sec. } 12, T \text {. } 1 \text { N., R. } 2 \text { W., } \\
\text { Grant County, approximately } 400 \mathrm{ft} \\
\text { downstream from country road bridge, } \\
\text { just downstream from tributary at } \\
\text { Louisburg, Wis. }\end{array}$ & 2.19 & $\begin{array}{l}\text { Oct. } 16,1975 \\
\text { Oct. } 27,1976 \\
\text { June } 21,1977\end{array}$ & $\begin{array}{l}.62 \\
.42 \\
.28\end{array}$ & .29 & .18 & c \\
\hline GP2A & $\begin{array}{l}\text { East Fork } \\
\text { Galena River }\end{array}$ & $\begin{array}{l}\text { SW } 1 / 4 \text { NE } 1 / 4 \text { sec. } 33, T .1 \text { N., R. } 2 \text { E., } \\
\text { Lafayette County, at bridge on } \\
\text { country road, } 4.0 \text { mi southeast } \\
\text { of New Diggings, Wis. }\end{array}$ & .83 & $\begin{array}{l}\text { Oct. } 15,1975 \\
\text { Oct. } 27,1976 \\
\text { June } 22,1977\end{array}$ & $\overleftarrow{2}_{0}^{3.97}$ & $<.01$ & 0 & $c$ \\
\hline GP3A & Betal Hollow & $\begin{array}{l}\text { NW } 1 / 4 S W 1 / 4 \text { sec. } 4, T .1 \text { N., R. } 2 \text { W., } \\
\text { Grant County, at culvert on country } \\
\text { road, } 1.1 \text { mi west of Kieler, Wis. }\end{array}$ & 1.42 & $\begin{array}{ll}\text { Oct. } 16,1975 \\
\text { Oct. } 27,1976 \\
\text { June } 21,1977\end{array}$ & $\begin{array}{l}.68 \\
.31 \\
.28\end{array}$ & .25 & .15 & c \\
\hline
\end{tabular}


Table 11. Grant-Platte River basin station locations and low-flow characteristics-Continued.

\begin{tabular}{|c|c|c|c|c|c|c|}
\hline $\begin{array}{l}\text { Station } \\
\text { number }\end{array}$ & Station location & $\begin{array}{l}\text { Drainage } \\
\text { area } \\
\left(\mathrm{mi}^{2}\right)\end{array}$ & Date & $\begin{array}{c}\text { Discharge } \\
\left(\mathrm{ft}^{3} / \mathrm{s}\right)\end{array}$ & $a_{7,2} a_{7,10}$ & $\begin{array}{l}\text { Accuracy } \\
\text { level }\end{array}$ \\
\hline GP4A Menominee River & $\begin{array}{l}\text { NWY SWY sec. } 22, \text { T. } 1 \text { N., } R .2 \text { W., } \\
\text { Grant County, at bridge on country } \\
\text { road, } 2.6 \text { mi south of Kleler, Wis. }\end{array}$ & 12.0 & $\begin{array}{l}\text { Oct. } 16,1976 \\
\text { Oct. } 27,1976 \\
\text { June } 21,1977\end{array}$ & $\begin{array}{l}3.13 \\
1.77 \\
1.02\end{array}$ & 0.56 & c \\
\hline
\end{tabular}

1 Based on 1971 report by Wisconsin Department of Natural Resources (DNR). Site lettered "A" was not included in 1971 DNR report.

2 Streambed is dry.

3 Additional discharge measurements are available.

${ }^{4}$ Negligible discharge-water ponded, unable to measure velocity.

$\mathbf{5}_{\text {No estimate possible-discharge is primarily effluent. }}$ 
Table 12. La Crosse River basin station locations and low-flow characteristics.

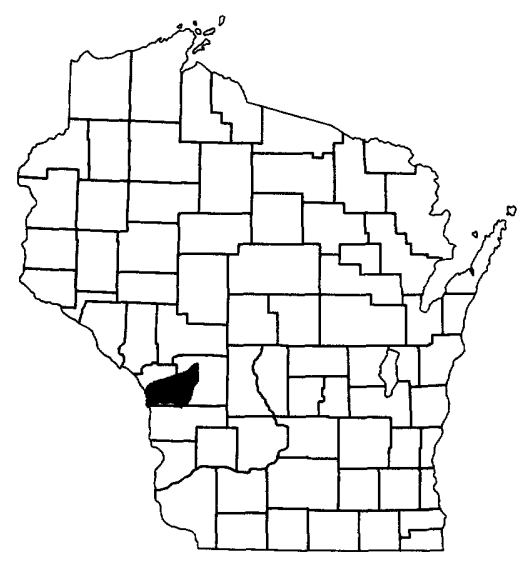

\section{La Crosse River basin}

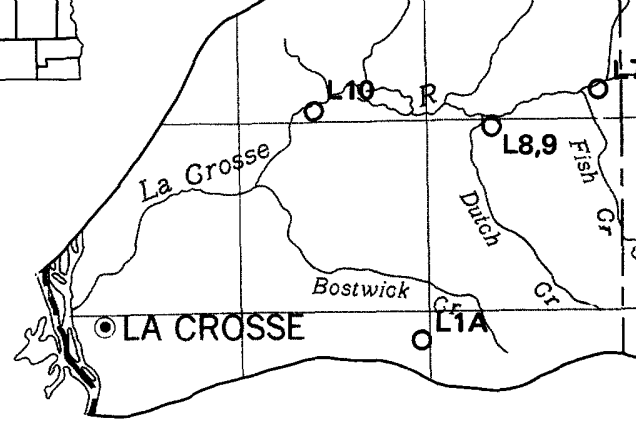

\section{Base from U.S. Geological Survey}

State base map, 1968

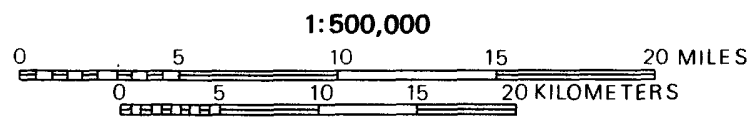

\begin{tabular}{|c|c|c|c|c|c|c|c|c|}
\hline $\begin{array}{l}\text { Station } \\
\text { number }\end{array}$ & ${ }_{\text {Stream }}^{1}$ & Station location & $\begin{array}{l}\text { Drainage } \\
\text { area } \\
\left(\mathrm{mi}^{2}\right)\end{array}$ & Date & $\begin{array}{c}\text { Discharge } \\
\left(\mathrm{ft}^{3} / \mathrm{s}\right)\end{array}$ & $\begin{array}{c}Q_{7,2} \\
\left(\mathrm{ft}^{3 / \mathrm{s}}\right)\end{array}$ & $\begin{array}{l}Q_{7,10} \\
\left(f^{3} / s\right)\end{array}$ & $\begin{array}{c}\text { Accuracy } \\
\text { level }\end{array}$ \\
\hline L4 & La Crosse River & $\begin{array}{l}S E^{1} / 4 N^{1} 1 / 4 \text { sec. } 27, T .18 \text { N., R. } 3 \text { W., } \\
\text { Monroe County, on country road, } \\
3.7 \text { mi northeast of Angelo, Wis. }\end{array}$ & 59.7 & $\begin{array}{l}\text { Aug. } 13,1970 \\
\text { Aug. } 10,1972 \\
\text { June } 27,1973 \\
\text { Sept. 11, } 1973 \\
\text { Aug. } 19,1976\end{array}$ & $\begin{array}{c}58.3 \\
60.5 \\
103 \\
72 \\
46.2\end{array}$ & 41 & 31 & c \\
\hline $\begin{array}{l}\angle 5 \\
6\end{array}$ & La Crosse River & $\begin{array}{l}\text { NE } 1 / 4 S^{S} E^{1} / 4 \text { sec. } 23, T .17 \text { N., R. } 4 \text { W., } \\
\text { Monroe County, upstream from sewage- } \\
\text { treatment plant, at Sparta, Wis. }\end{array}$ & 168 & $\begin{array}{l}\text { June } 27,1973 \\
\text { Sept. 11, } 1973 \\
\text { Aug. } 18,1976 \\
\text { Aug. } 8,1977\end{array}$ & $\begin{array}{l}223 \\
178 \\
104 \\
104\end{array}$ & 84 & 63 & c \\
\hline L7 & La Crosse River & $\begin{array}{l}\text { NW1/4NE1/4 sec. } 36, \text { T. } 17 \text { N., R. } 5 \text { W., } \\
\text { La Crosse County, on County Trunk } \\
\text { J, } 0.5 \text { mi north of Rockland, Wis. }\end{array}$ & 285 & $\begin{array}{l}\text { Aug. } 13,1970 \\
\text { Aug. } 10,1972 \\
\text { June } 26,1973 \\
\text { Sept. } 11,1973 \\
\text { Aug. } 18,1976\end{array}$ & $\begin{array}{l}178 \\
195 \\
338 \\
236 \\
167\end{array}$ & 120 & 87 & c \\
\hline $\begin{array}{l}\text { L8, } \\
9\end{array}$ & La Crosse River & $\begin{array}{l}\text { SE } 1 / 4 \text { SE } 1 / 4 \text { sec. } 32, T .17 \text { N., R. } 5 \text { W., } \\
\text { La Crosse County, just downstream } \\
\text { from Dutch Creek, } 0.6 \text { mi north } \\
\text { of Bangor, Wis. }\end{array}$ & 332 & $\begin{array}{l}\text { Aug. } 18,1976 \\
\text { Aug. } 8,1977 \\
\text { Sept. } 13,1977 \\
\text { Sept. } 11,1978\end{array}$ & $\begin{array}{l}192 \\
191 \\
172 \\
210\end{array}$ & 140 & 100 & c \\
\hline L10 & La Crosse River & $\begin{array}{l}\text { SW1/4SE } 1 / 4 \text { sec. } 32, \text { T. } 17 \text { N., R. } 6 \text { W., } \\
\text { La Crosse County, at town road, } \\
1.9 \text { mi west of West Salem, Wis. }\end{array}$ & 398 & & & 160 & 116 & a \\
\hline
\end{tabular}


Table 12. LaCrosse River basin station locations and low-flow characteristics-Continued.

\begin{tabular}{|c|c|c|c|c|c|c|c|c|}
\hline $\begin{array}{l}\text { Station } \\
\text { numbe }\end{array}$ & $\mathrm{n}^{1} \underset{\text { name }}{\text { Stream }}$ & Station location & $\begin{array}{l}\text { Drainage } \\
\text { area } \\
\left(\mathrm{mi}^{2}\right)\end{array}$ & Date & $\begin{array}{l}\text { Discharge } \\
\left(\mathrm{ft}^{3} / \mathrm{s}\right)\end{array}$ & $\begin{array}{l}Q_{7,2} Q_{7} \\
\left(\mathrm{ft}^{3} / \mathrm{s}\right)(\mathrm{ft}\end{array}$ & 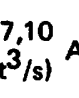 & $\begin{array}{c}\text { Accuracy } \\
\text { level }\end{array}$ \\
\hline \multirow[t]{2}{*}{ L11 } & $\begin{array}{l}\text { Little La Crosse } \\
\text { River }\end{array}$ & $\begin{array}{l}\text { NWY } / \text { SW1/ sec. } 30, \text { T. } 15 \text { N., R. } 3 \text { W., } \\
\text { Monroe County, at sewage-treatment } \\
\text { plant, at Cashton, Wis. }\end{array}$ & 0.97 & $\because$ & & & & \\
\hline & $\begin{array}{l}\text { Little Lo Crosse } \\
\text { River }\end{array}$ & $\begin{array}{l}\text { NWY/ANEY/4 sec. } 24, \text { T. } 15 \text { N., R. } 4 \text { W., } \\
\text { Monroe County, at State Highway } 27 \text {, } \\
0.8 \text { mi northwest of Cashton, Wis. }\end{array}$ & 3.05 & $\begin{array}{l}\text { Aug. } 10,1972 \\
\text { June } 28,1973 \\
\text { Sept. 11, } 1973 \\
\text { Aug. } 18,1976\end{array}$ & $\begin{array}{c}0.78 \\
2.03 \\
2.22 \\
.92\end{array}$ & 0.53 & 0.38 & c \\
\hline L12 & $\begin{array}{l}\text { Little La Crosse } \\
\text { River }\end{array}$ & $\begin{array}{l}\text { SEYNNE1/4 sec. 6, T. } 15 \text { N., R. } 3 \text { W., } \\
\text { Monroe County, at County Trunk F, } \\
\text { at Melvina, Wis. }\end{array}$ & 18.4 & $\begin{array}{l}\text { Aug. } 10,1972 \\
\text { June } 28,1973 \\
\text { Sept. 11, } 1973 \\
\text { Aug. } 18,1976\end{array}$ & $\begin{array}{l}6.37 \\
12.3 \\
12.7 \\
4.80\end{array}$ & 3.7 & 2.7 & c \\
\hline L1A & $\begin{array}{l}\text { St. Joseph } \\
\text { Coulee tributary }\end{array}$ & $\begin{array}{l}\text { NWYNEY// sec. 12, T. } 15 \text { N., R. } 6 \text { W., } \\
\text { La Crosse County, at bridge on } \\
\text { private road, } 0.8 \text { mi north of } \\
\text { St. Joseph, Wis. }\end{array}$ & .49 & $\begin{array}{l}\text { Aug. } 18,1976 \\
\text { Aug. } 9,1977 \\
\text { Sept. 13, } 1977 \\
\text { Sept. } 11,1978\end{array}$ & $\begin{array}{l}.39 \\
.28 \\
.36 \\
.45\end{array}$ & .17 & .13 & c \\
\hline
\end{tabular}

${ }^{1}$ Based on 1971 report by Wisconsin Department of Natural Resourcas (DNR). Site lettered "A" was not included in 1971 DNR report. 
Table 13. Manitowoc River basin station locations and low-flow characteristics.

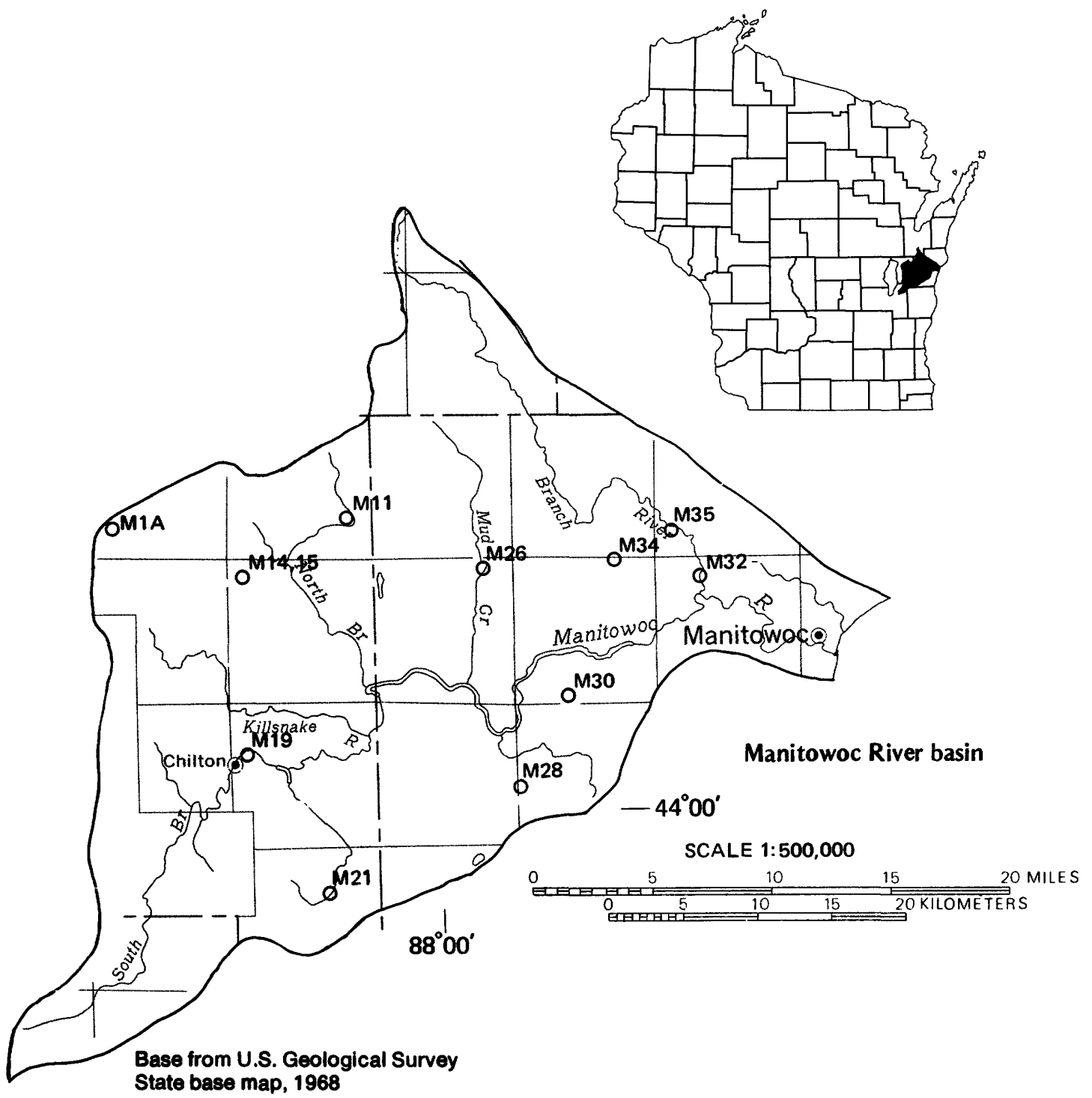

\begin{tabular}{|c|c|c|c|c|c|}
\hline $\begin{array}{l}\text { Station } \\
\text { number }\end{array}$ & Station location & $\begin{array}{c}\text { Drainage } \\
\text { area } \\
\left(\mathrm{mi}^{2}\right)\end{array}$ & Date & $\begin{array}{c}\text { Discharge } \\
\left(\mathrm{ft}^{3} / \mathrm{s}\right)\end{array}$ & $\begin{array}{cc}0_{7,2} & Q_{7,10} \\
\left(\mathrm{ft}^{3} / \mathrm{s}\right) & \left(\mathrm{ft}^{3} / \mathrm{s}\right) \\
\text { Accuracy } \\
\text { level }\end{array}$ \\
\hline
\end{tabular}

M11 Spring Creek

NWY $/ 4$ SE $/ 4$ sec. 26, T. 20 N., R. 20 E.

Calumet County, at sewage-treatment

plant, $0.3 \mathrm{mi}$ downstream from

State Highway 114, at Brillion, Wis.
7.42 July 17,1972

Oct. 11, 1972

July 31,1973

Sept. 5, 1974

Aug. 13, 1975

Sept. 30, 1976

1.70
1.74
.92
.84
.95
.44


Table 13. Manitowoc River basin station locations and low-flow characteristics-Continued.

\begin{tabular}{|c|c|c|c|c|c|c|c|c|}
\hline $\begin{array}{l}\text { Station } \\
\text { number }\end{array}$ & ${ }_{\text {name }}^{1}$ Stream & Station location & $\begin{array}{l}\text { Drainage } \\
\text { area } \\
\left(\mathrm{mi}^{2}\right)\end{array}$ & Date & $\begin{array}{c}\text { Discharge } \\
\left(\mathrm{ft}^{3} / \mathrm{s}\right)\end{array}$ & $\begin{array}{l}\mathrm{O}_{7,2} \mathrm{O}_{7} \\
\left(\mathrm{ft}^{3} / \mathrm{s}\right)\left(\mathrm{ft}^{3}\right.\end{array}$ & & $\begin{array}{l}\text { Accuracy } \\
\text { level }\end{array}$ \\
\hline \multirow[t]{2}{*}{$\begin{array}{c}\text { M14, } \\
15\end{array}$} & $\begin{array}{l}\text { North Branch } \\
\text { Manitowoc River } \\
\text { tributary }\end{array}$ & $\begin{array}{l}\text { SW } 1 / 4 \text { SE } 1 / 4 \text { sec. } 6, \text { T. } 19 \text { N., R. } 20 \text { E., } \\
\text { Calumet County, at sewage-treatment } \\
\text { plant, in Hilbert, Wis. }\end{array}$ & 3.24 & Aug. 12, 1975 & ${ }^{2} 0$ & 0 & 0 & c \\
\hline & $\begin{array}{l}\text { North Branch } \\
\text { Manitowoc River } \\
\text { tributary }\end{array}$ & $\begin{array}{l}\text { SE1//SE1/4 sec. } 6, \text { T. } 19 \text { N., R. } 20 \text { E., } \\
\text { Calumet County, at culvert on Irish } \\
\text { Road, } 0.9 \text { mi east of Hilbert, Wis. }\end{array}$ & 4.16 & $\begin{array}{l}\text { July } 17,1972 \\
\text { Oct. } 11,1972 \\
\text { July } 31,1973 \\
\text { Sept. } 5,1974 \\
\text { Aug. } 12,1975 \\
\text { Sept. } 30,1976\end{array}$ & $\begin{array}{l}.33 \\
.68 \\
.17 \\
.32 \\
.47 \\
0\end{array}$ & $\underline{5}$ & $\underline{5}$ & \\
\hline M19 & $\begin{array}{l}\text { South Branch } \\
\text { Manitowoc River }\end{array}$ & $\begin{array}{l}\text { NW } 1 / 4 \text { NW1/4 sec. } 17, \text { T. } 18 \text { N., R. } 20 \text { E., } \\
\text { Calumet County, at town road, } \\
1.1 \text { mi northeast of Chilton, Wis. }\end{array}$ & 75.2 & $\begin{array}{l}\text { July } 17,1972 \\
\text { Oct. } 10,1972 \\
\text { July } 31,1973 \\
\text { Sept. } 4,1974 \\
\text { Aug. } 12,1975 \\
\text { Oct. } 1,1976 \\
\text { July } 13,1977\end{array}$ & $\begin{array}{l}38.66 \\
54.5 \\
5.19 \\
5.53 \\
.73 \\
.27 \\
5.58\end{array}$ & .37 & .05 & b \\
\hline M21 & $\begin{array}{l}\text { Pine Creek } \\
\text { tributary }\end{array}$ & $\begin{array}{l}\text { SW } 1 / 4 \text { SW } 1 / 4 \text { sec. } 11, \text { T. } 17 \text { N., R. } 20 \text { E., } \\
\text { Calumet County, at sewage-treatment } \\
\text { plant, in New Holstein, Wis. }\end{array}$ & 3.62 & $\begin{array}{l}\text { July } 17,1972 \\
\text { Oct. } 10,1972 \\
\text { July } 31,1973 \\
\text { Sept. } 4,1974 \\
\text { Aug. } 12,1975 \\
\text { Oct. } 1,1976\end{array}$ & $\begin{array}{l}.04 \\
.22 \\
.08 \\
2_{0}^{.06} \\
2_{0}^{0}\end{array}$ & 0 & 0 & c \\
\hline \multirow[t]{2}{*}{ M26 } & Mud Creek & $\begin{array}{l}\text { SW1/4SW1/4 sec. } 2, \text { T. } 19 \text { N., R. } 21 \text { E., } \\
\text { Manitowoc County, at town road, } \\
1.0 \text { mi south of Reedsville, Wis. }\end{array}$ & 38.7 & $\begin{array}{l}\text { July } 18,1972 \\
\text { Oct. } 11,1972 \\
\text { Aug. } 1,1973 \\
\text { Nov. } 13,1973 \\
\text { Sept. } 5,1974 \\
\text { Aug. } 13,1975 \\
\text { Sept. } 30,1976 \\
\text { July } 13,1977\end{array}$ & $\begin{array}{l}3.40 \\
6.28 \\
1.85 \\
6.98 \\
.86 \\
.63 \\
.48 \\
.26\end{array}$ & .32 &. .12 & b \\
\hline & Mud Creek & $\begin{array}{l}\text { SW } 1 / 4 N E 1 / 4 \text { sec. } 2, T .19 \text { N., R. } 21 \text { E., } \\
\text { Manitowoc County, just upstream from } \\
\text { new sewage-treatment plant, in } \\
\text { Reedsville, Wis. }\end{array}$ & & $\begin{array}{l}\text { Aug. } 13,1975 \\
\text { Sept. } 30,1976 \\
\text { July } 13,1977\end{array}$ & $\begin{array}{l}.42 \\
.12 \\
.11\end{array}$ & .11 & .04 & c \\
\hline M28 & $\begin{array}{l}\text { Tributary to } \\
\text { tributary to } \\
\text { Manitowoc River } \\
\text { tributery }\end{array}$ & $\begin{array}{l}\text { NE } 1 / 4 \text { SE } 1 / 4 \text { sec. } 24, T .18 \text { N., R. } 21 \text { E., } \\
\text { Manitowoc County, et sewage-treetment } \\
\text { plant, at St. Nazianz, Wis. }\end{array}$ & .38 & $\begin{array}{l}\text { July } 18,1972 \\
\text { Oct. } 10,1972 \\
\text { Aug. } 1,1973 \\
\text { Sept. } 4,1974 \\
\text { Aug. } 13,1975 \\
\text { Oct. } 1,1976\end{array}$ & $\begin{array}{l}.06 \\
.03 \\
.28 \\
.12 \\
.02 \\
.04\end{array}$ & .01 & $<.01$ & c \\
\hline M30 & $\begin{array}{l}\text { Manitowoc River } \\
\text { tributary }\end{array}$ & $\begin{array}{l}\text { SE } 1 / 4 \text { SW } 1 / 4 \text { sec. } 32, \text { T. } 19 \text { N., R. } 22 \text { E., } \\
\text { Manitowoc County, at sewage- } \\
\text { treatment plant, in Velders, Wis. }\end{array}$ & 4.95 & $\begin{array}{l}\text { July } 18,1972 \\
\text { Oct. } 10,1972 \\
\text { Aug. } 1,1973 \\
\text { Nov. } 13,1973 \\
\text { Sept. } 4,1974 \\
\text { Aug. } 13,1975 \\
\text { Aug. } 12,1976 \\
\text { Oct. } 1,1976\end{array}$ & $\begin{array}{l}.62 \\
2.08 \\
2_{0}^{1.00} \\
20 \\
20 \\
20\end{array}$ & 0 & 0 & c \\
\hline M32 & Branch River & $\begin{array}{l}\text { NWY } / 4 \text { NE } 1 / 4 \text { sec. } 8, \text { T. } 19 \text { N., R. } 23 \text { E., } \\
\text { Manitowoc County, at Branch River } \\
\text { Country Club sewage-treatment } \\
\text { plant, } 0.1 \text { mi upstream from town } \\
\text { road, } 0.6 \text { mi south of Branch, Wis. }\end{array}$ & 108 & $\begin{array}{l}\text { July } 18,1972 \\
\text { Oct. } 11,1972 \\
\text { Aug. } 1,1973 \\
\text { Sept. } 5,1974 \\
\text { Aug. } 14,1975 \\
\text { Sept. } 30,1976\end{array}$ & $\begin{array}{l}16.7 \\
22.7 \\
18.6 \\
11.5 \\
6.76 \\
7.82\end{array}$ & 5.6 & 3.5 & c \\
\hline \multirow[t]{2}{*}{ M34 } & $\begin{array}{l}\text { Branch River } \\
\text { tributary }\end{array}$ & $\begin{array}{l}\text { SW } 1 / 4 N^{1} 1 / 4 \text { sec. } 2, \text { T. } 19 \text { N., R. } 22 \text { E., } \\
\text { Manitowoc County, at sewage- } \\
\text { treetment plant, in Whitelaw, Wis. }\end{array}$ & .26 & & & & & \\
\hline & $\begin{array}{l}\text { Brench River } \\
\text { tributary }\end{array}$ & $\begin{array}{l}\text { SW1/4NW1/4 sec. } 2, \text { T. } 19 \text { N., R. } 22 \text { E., } \\
\text { Manitowoc County, town road, } 0.75 \mathrm{mi} \\
\text { west of Whitelaw, Wis. }\end{array}$ & 1.30 & $\begin{array}{l}\text { July } 18,1972 \\
\text { Oct. } 11,1972 \\
\text { Aug. } 1,1973 \\
\text { Sept. } 5,1974 \\
\text { Aug. } 12,1975 \\
\text { Sept. } 28,1976\end{array}$ & $\begin{array}{l}.06 \\
.53 \\
.02 \\
.0 \\
.06 \\
.04\end{array}$ & $<.01$ & $<.01$ & c \\
\hline
\end{tabular}


Table 13. Manitowoc River basin station locations and low-flow characteristics-Continued.

\begin{tabular}{|c|c|c|c|c|c|c|c|c|}
\hline $\begin{array}{l}\text { Station } \\
\text { number }\end{array}$ & Stream & Station location & $\begin{array}{l}\text { Drainage } \\
\text { area } \\
\left(\mathrm{mi}^{2}\right)\end{array}$ & Date & $\begin{array}{l}\text { Discharge } \\
\left(\mathrm{ft}^{3} / \mathrm{s}\right)\end{array}$ & $\begin{array}{l}\mathrm{Q}_{7,2} \mathrm{Q}_{7,1} \\
\left(\mathrm{ft}^{3} / \mathrm{s}\right)\left(\mathrm{ft}^{3}\right)\end{array}$ & 10 & $\begin{array}{c}\text { Accuracy } \\
\text { level }\end{array}$ \\
\hline \multirow[t]{3}{*}{ M35 } & $\begin{array}{l}\text { Branch River } \\
\text { tributary }\end{array}$ & $\begin{array}{l}\text { SEY } 1 / 4 W 1 / 4 \text { sec. } 36, \text { T. } 20 \text { N., R. } 22 \text { E., } \\
\text { Manitowoc County, at sewage-treatment } \\
\text { site for Maple Crest Sanitarium, } \\
0.9 \text { mi northeast of Whitelaw, Wis. }\end{array}$ & 0.01 & & & & & \\
\hline & $\begin{array}{l}\text { Branch River } \\
\text { tributary }\end{array}$ & $\begin{array}{l}\text { NE1/4NE1/4 sec. } 36, T .20 \text { N., R. } 22 \text { E., } \\
\text { Manitowoc County, at culvert on } \\
\text { County Trunk } H, 1.8 \text { mi northeast } \\
\text { of Whitelaw, Wis. }\end{array}$ & .96 & $\begin{array}{l}\text { July } 18,1972 \\
\text { Oct. } 11,1972 \\
\text { Aug. } 1,1973 \\
\text { Nov. } 13,1973 \\
\text { Sept. } 5,1974\end{array}$ & $\begin{array}{l}q_{0} .03 \\
.12 \\
.24 \\
2_{0}^{24}\end{array}$ & 0 & 0 & c \\
\hline & Branch River & $\begin{array}{l}\text { NEY/4NE1/4 sec. } 31, \text { T. } 20 \text { N., R. } 23 \text { E., } \\
\text { ManitowOc County, at bridge on country } \\
\text { road, } 1.7 \text { mi northwest of Branch, Wis. }\end{array}$ & & $\begin{array}{l}\text { Sept. } 5,1974 \\
\text { Aug. } 14,1975 \\
\text { Sept. 30,1976 } \\
\text { July } 13,1977\end{array}$ & $\begin{array}{l}7.15 \\
4.01 \\
4.41 \\
3.68\end{array}$ & 4.3 & 3.1 & c \\
\hline M1A & $\begin{array}{l}\text { North Branch } \\
\text { Manitowoc River }\end{array}$ & $\begin{array}{l}\text { NWY } / 4 \text { NEY/4 sec. } 32, \text { T. } 20 \text { N., R. } 19 \text { E., } \\
\text { Calumet County, at culvert on } \\
\text { County Trunk B, at Sherwood, Wis. }\end{array}$ & 3.09 & $\begin{array}{l}\text { Oct. } 31,1975 \\
\text { July } 14,1976 \\
\text { Sept. 16, } 1976 \\
\text { Oct. } 1,1976 \\
\text { Oct. } 15,1976 \\
\text { Nov. } 1,1976 \\
\text { May } 17,1977\end{array}$ & $\begin{array}{l}.19 \\
.04 \\
.05 \\
.04 \\
.05 \\
.07 \\
.14\end{array}$ & .04 & .02 & c \\
\hline
\end{tabular}

1Based on 1969 report by Wisconsin Department of Natural Resources (DNR). Site lettered "A" was not included in 1969 DNR report.

2 Streambed is dry.

3 Additional discharge measurements are available.

${ }^{4}$ Negligible discharge--water ponded, unable to measure velocity.

${ }^{5}$ No estimate possible--discharge is primarily effluent. 
Table 14. Menominee River basin station locations and low-flow characteristics.

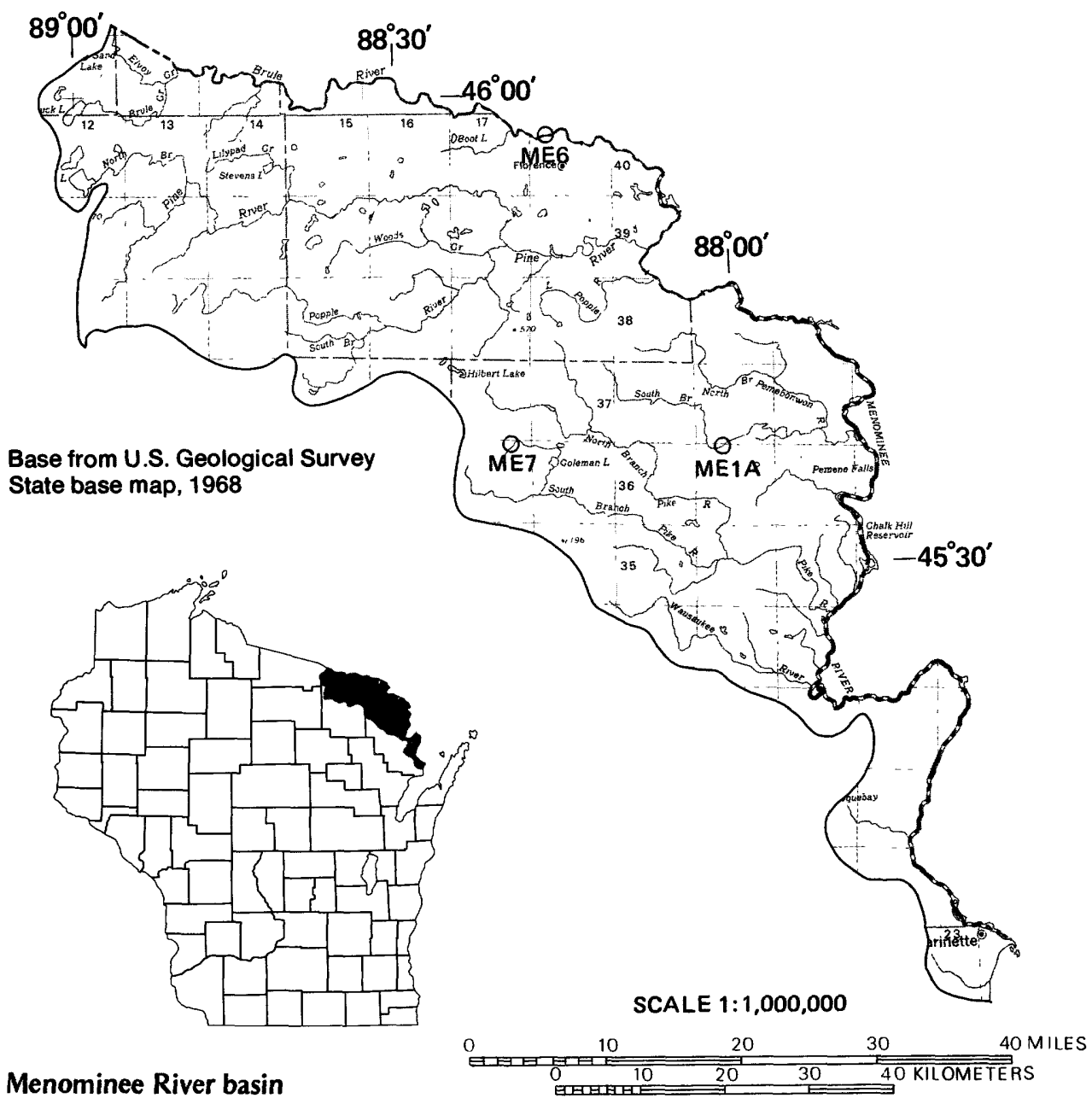

\begin{tabular}{|c|c|c|c|c|c|c|}
\hline $\begin{array}{l}\text { Station } \\
\text { number }\end{array}$ & $\underset{\text { Streame }}{\text { name }}$ & Station location & $\begin{array}{l}\text { Drainage } \\
\text { area } \\
\left(m i^{2}\right)\end{array}$ & Date & $\begin{array}{c}\text { Discharge } \\
\left(\mathrm{ft}^{3} / \mathrm{s}\right)\end{array}$ & $\begin{array}{ll}\mathrm{Q}_{7,2} & \mathrm{Q}_{7,10} \\
\left(\mathrm{ft}^{3} / \mathrm{s}\right)\left(\mathrm{ft}^{3} / \mathrm{s}\right) & \text { Accuracy } \\
\text { level } & \end{array}$ \\
\hline
\end{tabular}

ME6 Brule River tributary

Brule River ME7

Chemical Creek
SE $/ 4 S W \%$ sec. 16, T. 40 N., R. 18 E.

Florence County, at bridge on country road, $1.0 \mathrm{mi}$ north of Florence, Wis.

SE1/4SE\% sec. 11, T. 41 N., R. 32 W., Michigan meridian, on left bank $40 \mathrm{ft}$ upstream from highway bridge, $1.0 \mathrm{mi}$ upstream from Paint River, $2.6 \mathrm{mi}$ north of Florence, Wis.

SW1/4NE $1 / 4$ sec. 3, T. 36 N., R. 17 E., Marinette County, just upstream from sewage-treatment plant, at Goodman, Wis.

ME1A South Brench SW $1 / 4$ NW $1 / 4$ sec. 4 , T. 36 N., R. 20 E., Pemebonwon River Marinette County, at bridge on U.S. Highway $8,1.5 \mathrm{mi}$ southwest of Pembine, Wis.
0.60
4.57 Aug. 1, 1972

June 27,1973

Aug. 29,1973

Oct. 1,1974

July 8,1975

Aug. 5, 1975

Aug. 31,1976

Oct. 13, 1976

40.9
Oct. 3, 1974
July 8,1975
Aug. 5, 1975
Oct. 13, 1976
24.4
19.6
13.9

2.16

5.32

2.65

1.52

.54

1.07

.93

'Based on 1969 report by Wisconsin Department of Natural Resources (DNR). Site lettered "A" was not included in 1969 DNR report. 
Table 15. Milwaukee River basin station locations and low-flow characteristics.
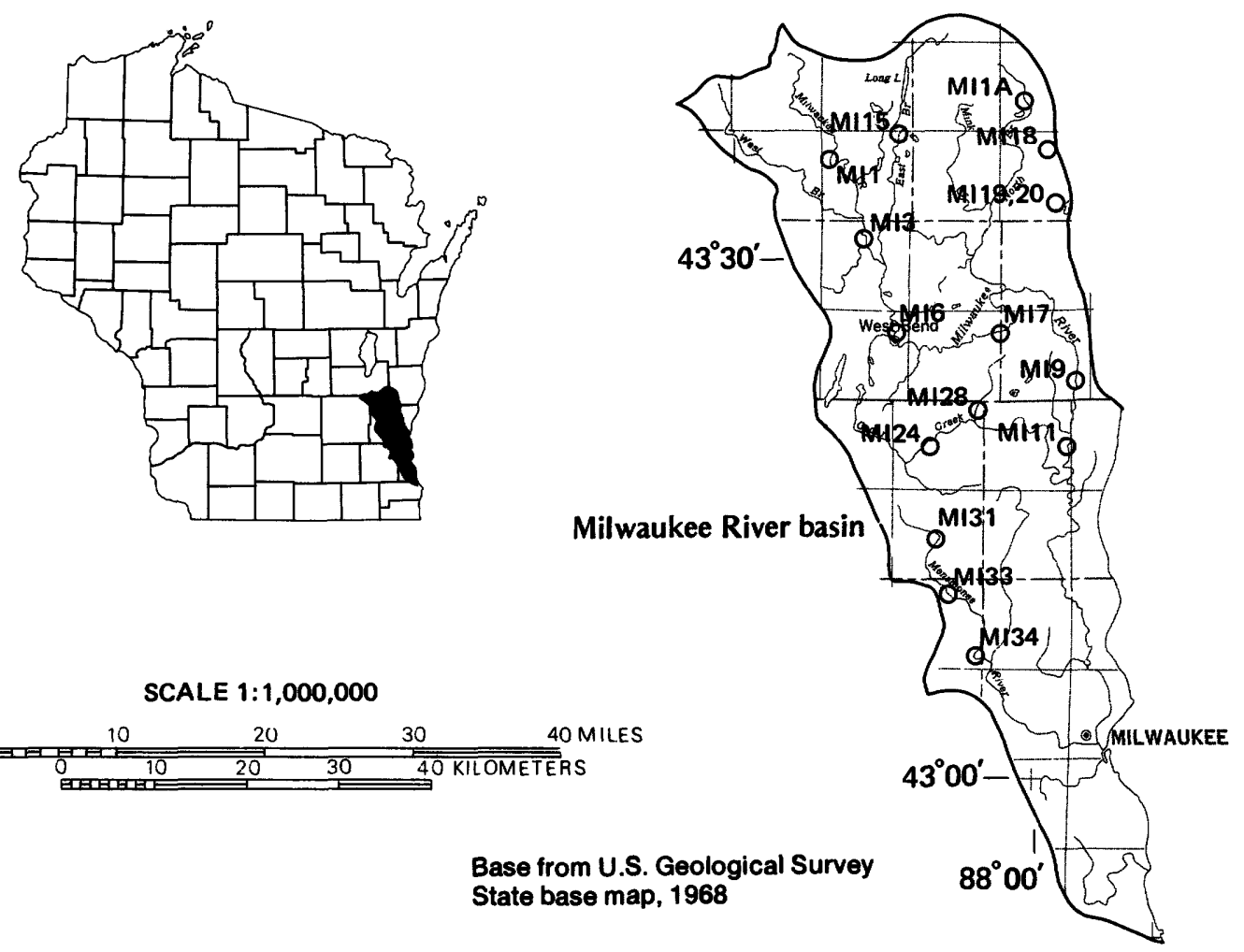

\begin{tabular}{|c|c|c|c|c|c|c|c|c|}
\hline $\begin{array}{l}\text { Station } \\
\text { number }\end{array}$ & 1 Stream & Station location & $\begin{array}{l}\text { Drainage } \\
\text { area } \\
\left(\mathrm{mi}^{2}\right)\end{array}$ & Date & $\begin{array}{c}\text { Discharge } \\
\left(\mathrm{ft}^{3} / \mathrm{s}\right)\end{array}$ & $\begin{array}{ll}\mathrm{Q}_{7,2} & \mathrm{Q}_{7} \\
\left(\mathrm{ft}^{3} / \mathrm{s}\right)(\mathrm{ft}\end{array}$ & $\hat{S}_{/ \mathrm{s})}^{10} \mathrm{Ac}$ & $\begin{array}{l}\text { Accuracy } \\
\text { level }\end{array}$ \\
\hline MI1 & Milwaukee River & $\begin{array}{l}\text { NE } 1 / 4 \text { SE } 1 / 4 \text { sec. } 7, T .13 \text { N., R. } 19 \text { E., } \\
\text { Fond du Lac County, at bridge on } \\
\text { private road, } 0.7 \text { mi northeast } \\
\text { from junction of State Highway } 67 \\
\text { and County Trunk Y, in Campbellsport, } \\
\text { Wis. }\end{array}$ & 51.4 & & & 0.24 & 0.07 & b \\
\hline M13 & Milwaukee River & $\begin{array}{l}\text { NE } 1 / 4 \text { SE } 1 / 4 \text { sec. } 9, \text { T. } 12 \text { N., R. } 19 \text { E., } \\
\text { Washington County, at dam } 0.25 \mathrm{mi} \\
\text { downstream from State Highway } 28 \text {, } \\
\text { at Kewaskum, Wis. }\end{array}$ & 138 & & & 7.6 & 1.3 & a \\
\hline \multirow[t]{2}{*}{ MI6 } & Milwaukee River & $\begin{array}{l}\text { SE } 1 / 4 N E 1 / 4 \text { sec. } 14, T .11 \text { N., R. } 19 \text { E., } \\
\text { Wash ington County, at bridge on } \\
\text { city street, at West Bend, Wis. }\end{array}$ & 236 & $\begin{array}{l}\text { July } 26,1972 \\
\text { June } 14,1973 \\
\text { Aug. } 14,1973 \\
\text { Oct. } 10,1975 \\
\text { Sept. } 30,1976\end{array}$ & $\begin{array}{c}44.9 \\
175 \\
48.7 \\
35.7 \\
18.9\end{array}$ & 14 & 5.1 & c \\
\hline & Milwaukee River & $\begin{array}{l}\text { SW } 1 / 4 N W 1 / 4 \text { sec. } 13, \text { T. } 11 \text { N., R. } 19 \text { E., } \\
\text { Washington County, at sewage- } \\
\text { treatment plant, at West Bend, Wis. }\end{array}$ & 237 & Oct. 10,1975 & 37.9 & & & \\
\hline Mi7 & Milwaukee River & $\begin{array}{l}\text { SW } 1 / 4 \text { NE } 1 / \text { sec. } 12, \text { T. } 11 \text { N., R. } 20 \text { E., } \\
\text { Washington County, at County Trunk } \\
\text { MY, at Newburg, Wis. }\end{array}$ & 265 & $\begin{array}{l}\text { Oct. } 3,1968 \\
\text { June } 14,1973 \\
\text { Aug. } 14,1973 \\
\text { Oct. } 11,1975 \\
\text { Sept. } 28,1976 \\
\text { Sept. } 30,1976 \\
\text { Nov. } 12,1976\end{array}$ & $\begin{array}{c}50.9 \\
192 \\
58.3 \\
43.1 \\
25.3 \\
24.4 \\
36.5\end{array}$ & 21 & 8.9 & c \\
\hline
\end{tabular}


Table 15. Milwaukee River basin station locations and low-flow characteristics-Continued.

\begin{tabular}{|c|c|c|c|c|c|c|c|c|}
\hline $\begin{array}{l}\text { Station } \\
\text { number }\end{array}$ & Stream & Station location & $\begin{array}{l}\text { Drainage } \\
\text { area } \\
\left(\mathrm{mi}^{2}\right)\end{array}$ & Date & $\begin{array}{c}\text { Discharge } \\
\left(\mathrm{ft}^{3} / \mathrm{s}\right)\end{array}$ & $\begin{array}{l}\mathrm{Q}_{7,2} \mathrm{Q}_{7} \\
\left(\mathrm{ft}^{3} / \mathrm{s}\right)\left(\mathrm{ft}^{3}\right.\end{array}$ & & $\begin{array}{c}\text { Accuracy } \\
\text { level }\end{array}$ \\
\hline \multirow[t]{2}{*}{ MI9 } & Milwaukee River & $\begin{array}{l}\text { NW1/4NW1/4 sec. } 36, T \text {. } 11 \text { N., R. } 21 \text { E., } \\
\text { Ozaukee County, at bridge on State } \\
\text { Highway 33, at Saukville, Wis. }\end{array}$ & 457 & $\begin{array}{l}\text { June } 14,1973 \\
\text { Aug. } 14,1973 \\
\text { Oct. 11, 1975 } \\
\text { Sept. 28, 1976 }\end{array}$ & $\begin{array}{l}306 \\
118 \\
78.9 \\
60.2\end{array}$ & 46 & 22 & c \\
\hline & Milwaukee River & $\begin{array}{l}\text { NE } 1 / 4 S E 1 / 4 \text { sec. } 35, \text { T. } 11 \text { N., R. } 21 \text { E., } \\
\text { Ozaukee County, } 50 \text { ft upstream from } \\
\text { sewage-treatment plant, at Saukville, } \\
\text { Wis. }\end{array}$ & 461 & Oct. 3, 1968 & 72.4 & & & \\
\hline MI11 & Milwaukee River & $\begin{array}{l}\text { NW } 1 / 4 N E 1 / 4 \text { sec. } 25, T .10 \text { N., R. } 21 \text { E., } \\
\text { Ozaukee County, just upstream } \\
\text { from sewage-treatment plant, } \\
\text { at Grafton, Wis. }\end{array}$ & 476 & $\begin{array}{l}\text { Aug. 14, } 1973 \\
\text { Oct. 11, } 1975 \\
\text { Sept. 27, } 1976\end{array}$ & $\begin{array}{r}121 \\
80.9 \\
46.9\end{array}$ & 46 & 22 & c \\
\hline MI15 & $\begin{array}{l}\text { East Branch } \\
\text { Milwaukee River }\end{array}$ & $\begin{array}{l}\text { SE } 1 / 4 \text { SE } 1 / 4 \text { sec. } 2, T .13 \text { N., R. } 19 \text { E., } \\
\text { Fond du Lac County, at bridge on } \\
\text { County Trunk SS, } 2.3 \text { mi south of } \\
\text { Dundee, Wis. }\end{array}$ & 36.2 & $\begin{array}{l}\text { July } 26,1962 \\
\text { June 14, } 1973 \\
\text { Aug. 15, 1973 } \\
\text { Oct. 10, 1975 } \\
\text { Sept. 28, } 1976\end{array}$ & $\begin{array}{l}7.90 \\
25.4 \\
8.52 \\
7.15 \\
5.51\end{array}$ & 4.2 & 1.2 & c \\
\hline MI18 & $\begin{array}{l}\text { North Branch } \\
\text { Milwaukee River } \\
\text { tributary }\end{array}$ & $\begin{array}{l}\text { NW1/4NW1/4 sec. 2, T. } 13 \text { N., R. } 21 \text { E., } \\
\text { Sheboygan County, at bridge on } \\
\text { County Trunk W, } 1.0 \text { mi north of } \\
\text { Adell, Wis. }\end{array}$ & 4.23 & $\begin{array}{l}\text { July } 26,1972 \\
\text { Aug. 15, } 1973 \\
\text { Oct. 10, } 1975 \\
\text { Sept. 28, 1976 } \\
\text { July } 11,1977\end{array}$ & $\begin{array}{l}.88 \\
.76 \\
.46 \\
.08 \\
.45\end{array}$ & .08 & .01 & c \\
\hline $\begin{array}{l}\text { MI19, } \\
20\end{array}$ & Silver Creek & $\begin{array}{l}\text { SEY } 1 / 4 \text { SW } 1 / 4 \text { sec. } 27, \text { T. } 13 \text { N., R. } 21 \text { E., } \\
\text { Sheboygan County, at bridge on } \\
\text { State Highway } 144,0.7 \text { mi northwest } \\
\text { of Random Lake, Wis. }\end{array}$ & 11.7 & $\begin{array}{l}\text { July } 26,1972 \\
\text { June } 14,1973 \\
\text { Aug. 15, 1973 } \\
\text { Oct. 10, 1975 } \\
\text { Sept. 29, } 1976\end{array}$ & $\begin{array}{l}1.98 \\
6.04 \\
.58 \\
.74 \\
.40\end{array}$ & .30 & .10 & c \\
\hline MI24 & Cedar Creek & $\begin{array}{l}\text { NE1/4NE1/4 sec. } 20, T .10 \text { N., R. } 20 \text { E., } \\
\text { Washington County, at bridge on } \\
\text { State Highway } 60,1.2 \text { mi east of } \\
\text { Jackson, Wis. }\end{array}$ & 53.6 & $\begin{array}{l}\text { Sept. 16, } 1968 \\
\text { Sept. 17, } 1968 \\
\text { Oct. 2, } 1968 \\
\text { July } 25,2972 \\
\text { June } 13,1973 \\
\text { Aug. } 14,1973 \\
\text { Oct. 10, } 1975 \\
\text { Sept. 27, } 1976\end{array}$ & $\begin{array}{c}7.80 \\
7.29 \\
9.33 \\
20.2 \\
38.1 \\
16.7 \\
13.1 \\
9.41\end{array}$ & 3.5 & .94 & c \\
\hline M128 & $\begin{array}{l}\text { Cedar Creek } \\
\text { tributary }\end{array}$ & $\begin{array}{l}\text { NE1/4NW1/4 sec. 12, T. } 10 \mathrm{~N} \text {., R. } 20 \mathrm{E} ., \\
\text { Washington County, at bridge on } \\
\text { Pleasant Valley road, } 5.0 \mathrm{mi} \\
\text { northeast of Jackson, Wis. }\end{array}$ & 13.8 & $\begin{array}{l}\text { Sept. 13, } 1968 \\
\text { Oct. 10, } 1975 \\
\text { Sept. 27, } 1976 \\
\text { July 11, } 1977\end{array}$ & $\begin{array}{l}.60 \\
1.38 \\
.55 \\
.38\end{array}$ & .19 & .04 & c \\
\hline \multirow[t]{2}{*}{ M131 } & Menomonee River & $\begin{array}{l}\text { SWY/4 SWY/4 sec. 15, T. } 9 \text { N., R. } 20 \text { E., } \\
\text { Wash ington County, at bridge on } \\
\text { Freistadt Road, } 0.9 \text { mi northwest } \\
\text { of Germantown, Wis. }\end{array}$ & 11.3 & $\begin{array}{l}\text { June } 13,1973 \\
\text { Aug. } 14,1973 \\
\text { Oct. } 10,1975 \\
\text { Sept. } 27,1976\end{array}$ & $\begin{array}{l}2.69 \\
4.30 \\
40\end{array}$ & .05 & $<.01$ & c \\
\hline & Menomonee River & $\begin{array}{l}\text { SE } 1 / 4 N E 1 / 4 \text { sec. } 21, T .9 \text { N., R. } 20 \text { E., } \\
\text { Washington County, at sewage- } \\
\text { treatment plant, } 1.0 \text { mi west of } \\
\text { Germantown, Wis. }\end{array}$ & 17.4 & & & & & \\
\hline M133 & Menomonee River & $\begin{array}{l}\text { SW } 1 / 4 S E 1 / 4 \text { sec. } 33, T \text {. } 9 \text { N., R. } 20 \text { E., } \\
\text { Washington County, at County Trunk } Q \text {, } \\
1.2 \text { mi northwest of Menomonee Falls, } \\
\text { Wis. }\end{array}$ & 30.7 & & & 1.9 & .54 & $\mathbf{b}$ \\
\hline MI34 & Menomonee River & $\begin{array}{l}\text { SE1/4NE1/4 sec. } 36, \text { T. } 8 \text { N., R. } 20 \text { E., } \\
\text { Waukesha County, at bridge on 124th } \\
\text { Street, } 0.7 \text { mi north of post office, } \\
\text { in Butler, Wis. }\end{array}$ & 60.6 & $\begin{array}{l}\text { July } 25,1972 \\
\text { June } 13,1973 \\
\text { Aug. } 14,1973 \\
\text { Sept. 27, } 1976\end{array}$ & $\begin{array}{l}11.5 \\
25.0 \\
8.53 \\
4.24\end{array}$ & 3.7 & 1.1 & c \\
\hline MIIA & $\begin{array}{l}\text { North Branch } \\
\text { Milwaukee River }\end{array}$ & $\begin{array}{l}\text { NW1/4NE1/4 sec. } 29, \text { T. } 14 \text { N., R. } 21 \text { E., } \\
\text { Sheboygan County, at bridge on } \\
\text { State Highway } 28 \text {, at Cascade, Wis. }\end{array}$ & 7.48 & $\begin{array}{l}\text { Oct. } 10,1975 \\
\text { Sept. 28, } 1976 \\
\text { July 11, } 1977\end{array}$ & $\begin{array}{l}7.21 \\
6.22 \\
4.35\end{array}$ & 3.7 & 2.2 & c \\
\hline
\end{tabular}

${ }_{1}^{1}$ ased on 1968 report by Wisconsin Department of Natural Resources (DNR). Site lettered "A" was not included in 1968 DNR report.

${ }^{4}$ Negligible discharge--water ponded, unable to measure velocity. 
Table 16. Oconto River basin station locations and low-flow characteristics.

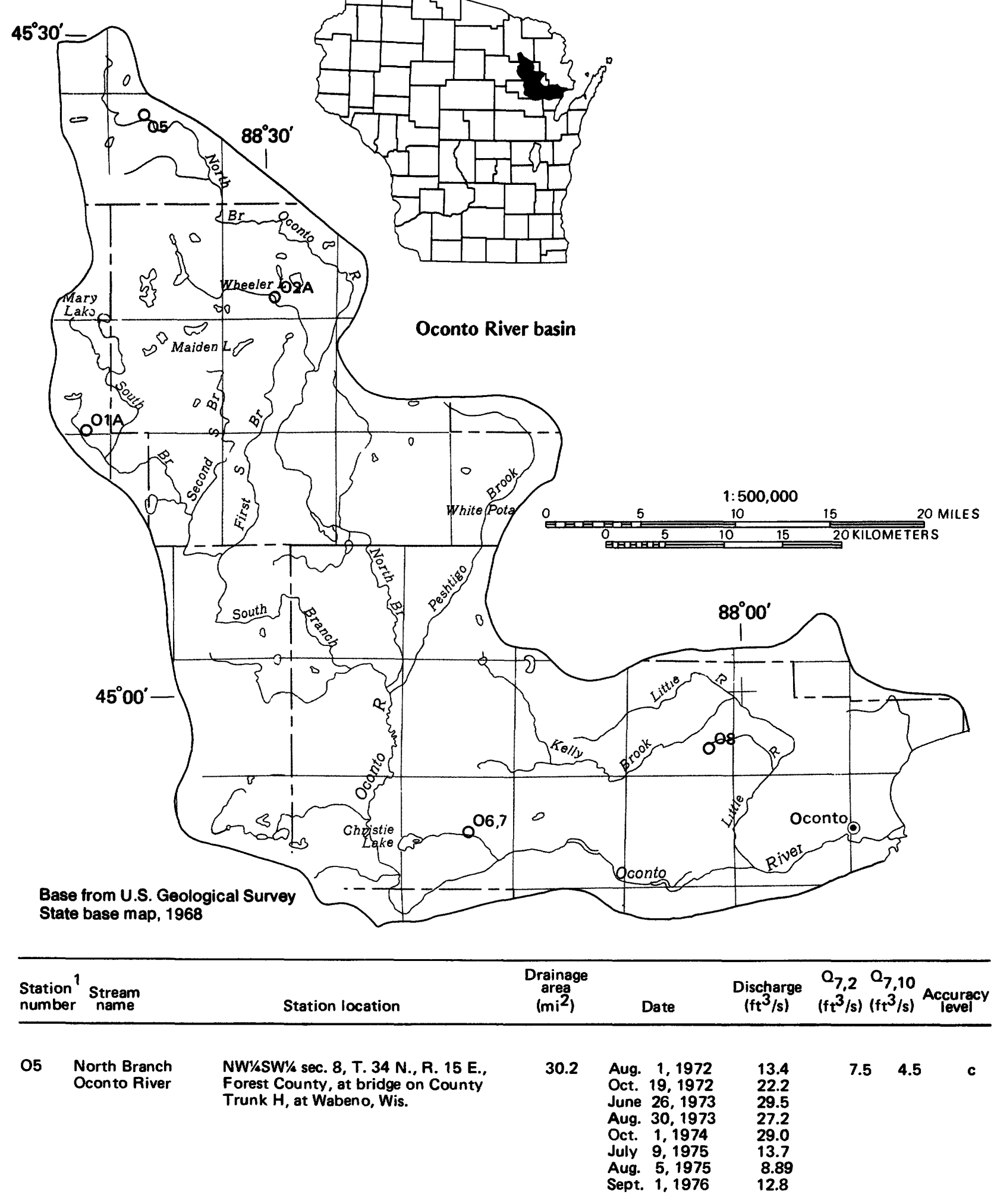


Table 16. Oconto River basin station locations and low-flow characteristics-Continued.

\begin{tabular}{|c|c|c|c|c|c|c|c|c|}
\hline $\begin{array}{l}\text { Station } \\
\text { number }\end{array}$ & Stream & Station location & $\begin{array}{l}\text { Drainage } \\
\text { area } \\
\left(\mathrm{mi}^{2}\right)\end{array}$ & Date & $\begin{array}{c}\text { Discharge } \\
\left(\mathrm{ft}^{3} / \mathrm{s}\right)\end{array}$ & $\begin{array}{l}Q_{7,2} Q_{7} \\
\left(\mathrm{ft}^{3} / \mathrm{s}\right)\left(\mathrm{ft}^{2}\right.\end{array}$ & $\begin{array}{l}7,10 \\
\left.t^{3} / s\right)\end{array}$ & $\begin{array}{c}\text { Accuracy } \\
\text { level }\end{array}$ \\
\hline O6, & Christie Brook & $\begin{array}{l}\text { NEKNW\%/ sec. 22, T. } 28 \text { N., R. } 18 \text { E., } \\
\text { Oconto County, at culvert on State } \\
\text { Highway 22, at Gillett, Wis. }\end{array}$ & 7.45 & $\begin{array}{lc}\text { Oct. } & 1,1974 \\
\text { July } & 7,1975 \\
\text { Aug. } & 4,1975 \\
\text { Aug. } & 30,1976 \\
\text { Oct. } 12,1976\end{array}$ & $\begin{array}{l}1.33 \\
1.65 \\
.50 \\
.23 \\
.31\end{array}$ & 0.41 & 0.17 & c \\
\hline \multirow[t]{2}{*}{08} & $\begin{array}{l}\text { Little River } \\
\text { tributary }\end{array}$ & $\begin{array}{l}\text { SEYSEY/ sec. } 27, \text { T. } 29 \text { N., R. } 20 \text { E., } \\
\text { Oconto County, at sewage-treatment } \\
\text { plant, at Lena, Wis. }\end{array}$ & 1.36 & 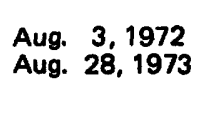 & ${ }^{2} 0$ & 0 & $\mathbf{0}$ & c \\
\hline & $\begin{array}{l}\text { Little River } \\
\text { tributary }\end{array}$ & $\begin{array}{l}\text { NEYSEY sec. 26. T. } 29 \text { N., R. } 20 \text { E., } \\
\text { Oconto County, at culvert on U.S. } \\
\text { Highway 141, at Lena, Wis. }\end{array}$ & 1.63 & $\begin{array}{l}\text { Aug. } 3,1972 \\
\text { Oct. } 18,1972 \\
\text { June } 25,1973 \\
\text { Aug. } 28,1973 \\
\text { Sept. } 30,1974 \\
\text { July } 10,1975 \\
\text { Aug. } 7,1975 \\
\text { Aug. } 30,1976\end{array}$ & $\begin{array}{l}{ }_{4}^{4} 0 \\
0 \\
1.56 \\
.02 \\
.85 \\
.67 \\
.45 \\
.25\end{array}$ & $\mathbf{0}$ & 0 & c \\
\hline $01 \mathrm{~A}$ & Dalton Creek & $\begin{array}{l}\text { SEY/\%E1/2 sec. } 2, \text { T. } 31 \text { N., R. } 14 \text { E., } \\
\text { Langlade County, at culvert on road } \\
\text { to fish hatchery, } 1.5 \text { mi east of } \\
\text { Langlade, Wis. }\end{array}$ & 10.6 & $\begin{array}{lc}\text { Oct. } & 1,1974 \\
\text { July } & 9,1975 \\
\text { Aug. } & 6,1975 \\
\text { Aug. } & 30,1976 \\
\text { Oct. } & 12,1976\end{array}$ & $\begin{array}{l}6.79 \\
3.58 \\
5.53 \\
1.44 \\
4.16\end{array}$ & 2.7 & 1.4 & c \\
\hline O2A & McCaslin Brook & $\begin{array}{l}\text { SW\%NEY sec. } 33 \text {, T. } 33 \text { N., R. } 16 \text { E., } \\
\text { Oconto County, at bridge on County } \\
\text { Trunk F, } 1.2 \text { mi west of Lakewood, } \\
\text { Wis. }\end{array}$ & 58.8 & 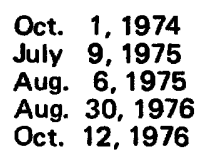 & $\begin{array}{l}25.3 \\
18.1 \\
15.5 \\
12.0 \\
13.2\end{array}$ & 14 & 9.8 & c \\
\hline
\end{tabular}

1 Based on 1969 report by Wisconsin Department of Natural Resources (DNR). Site lettered "A" was not included in 1969 DNR report.

2 Streambed is dry.

${ }^{4}$ Negligible discharge--water ponded, unable to measure velocity. 
Table 17. Pecatonica River basin station locations and low-flow characteristics.

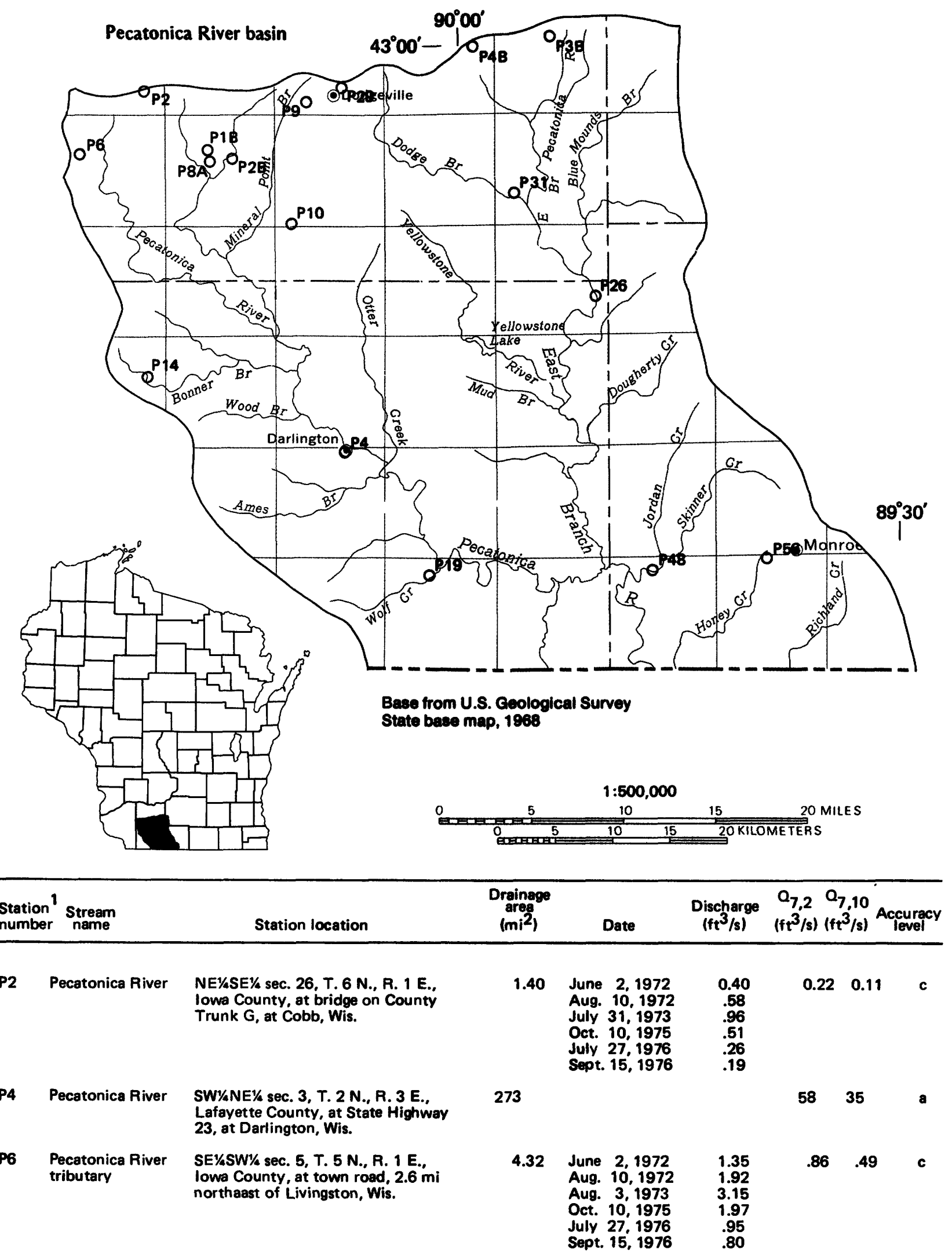


Table 17. Pecatonica River basin station locations and low-flow characteristics-Continued.

\begin{tabular}{|c|c|c|c|c|c|c|c|c|}
\hline $\begin{array}{l}\text { Station } \\
\text { number }\end{array}$ & ${ }^{1}$ Stream & Station location & $\begin{array}{l}\text { Drainage } \\
\text { area } \\
\left(m i^{2}\right)\end{array}$ & Date & $\begin{array}{l}\text { Discharge } \\
\left(\mathrm{ft}^{3} / \mathrm{s}\right)\end{array}$ & $\begin{array}{l}\mathrm{Q}_{7,2} \mathrm{Q}_{7} \\
\left(\mathrm{ft}^{3} / \mathrm{s}\right)\left(\mathrm{ft}^{3}\right.\end{array}$ & $\begin{array}{l}7,10 \\
3 / s)\end{array}$ & $\underset{\substack{\text { Accuracy } \\
\text { level }}}{\text { Actis }}$ \\
\hline \multirow[t]{2}{*}{ P8A } & $\begin{array}{l}\text { East Pecatonica } \\
\text { River tributary }\end{array}$ & $\begin{array}{l}\text { NW\%NW\% sec. 16, T. } 5 \text { N., R. } 2 \text { E., } \\
\text { lowa County, at bridge on State } \\
\text { Highway } 39 \text {, in Linden, Wis. }\end{array}$ & 2.79 & $\begin{array}{lc}\text { June } & 2,1972 \\
\text { Aug. } 10,1972 \\
\text { July } 31,1973 \\
\text { Oct. } 10,1975 \\
\text { July } 27,1976 \\
\text { Sept. } 15,1976\end{array}$ & $\begin{array}{l}.84 \\
.75 \\
2.54 \\
1.30 \\
.56 \\
.51\end{array}$ & 0.54 & .0 .28 & c \\
\hline & $\begin{array}{l}\text { East Pecatonica } \\
\text { River tributary }\end{array}$ & $\begin{array}{l}\text { SW1/NW1/ sec. 16, T. } 5 \text { N., R. } 2 \text { E., } \\
\text { lowa County, at sewage-treatment } \\
\text { plant, in Linden, Wis. }\end{array}$ & 3.11 & & & & & \\
\hline \multirow[t]{2}{*}{ P9 } & Pedlar Creek & $\begin{array}{l}\text { SW\%SE } \% \text { sec. } 26, \text { T. } 6 \text { N., R. } 2 \text { E., } \\
\text { lowa County, at bridge on town } \\
\text { road, } 4.6 \text { mi west of Dodgeville, } \\
\text { Wis. }\end{array}$ & 1.46 & $\begin{array}{l}\text { June } 2,1972 \\
\text { Aug. } 10,1972 \\
\text { July } 31,1973 \\
\text { Oct. } 10,1975 \\
\text { July } 27,1976 \\
\text { Sept. } 15,1976\end{array}$ & $\begin{array}{l}.44 \\
1.72 \\
1.64 \\
1.21 \\
.54 \\
.40\end{array}$ & .48 & .27 & c \\
\hline & Pedlar Creak & $\begin{array}{l}\text { SE } 1 / \text { NWW sec. } 35, \text { T. } 6 \text { N., R. } 2 \text { E., } \\
\text { lowa County, at sewage-treatment } \\
\text { plant, } 4.8 \text { mi west of Dodgeville, } \\
\text { Wis. }\end{array}$ & 1.67 & & & & & \\
\hline \multirow[t]{2}{*}{ P10 } & Brewery Creek & $\begin{array}{l}\text { SW1/4NE1/ sec. 6. T. } 4 \text { N., R. } 3 \text { E., } \\
\text { lowa County, at bridge on town } \\
\text { road, in Mineral Point, Wis. }\end{array}$ & 6.74 & $\begin{array}{l}\text { June } 2,1972 \\
\text { Aug. } 10,1972 \\
\text { July } 31,1973 \\
\text { Oct. } 9,1975 \\
\text { July } 27,2976 \\
\text { Sept. } 14,1976 \\
\text { June } 23,1977\end{array}$ & $\begin{array}{l}1.93 \\
1.53 \\
4.83 \\
2.57 \\
1.35 \\
1.06 \\
.83\end{array}$ & 1.2 & .68 & c \\
\hline & Brewery Creek & $\begin{array}{l}\text { SW\%NE1/4 sec. 6, T. } 4 \text { N., R. } 3 \text { E., } \\
\text { lowa County, at bridge on town } \\
\text { road, in Mineral Point, Wis. }\end{array}$ & 6.79 & & & & & \\
\hline P14 & Bonner Branch & $\begin{array}{l}\text { SEYNE } 1 / \text { sec. } 14, \text { T. } 3 \text { N., R. } 1 \text { E., } \\
\text { Lafayette County, at bridge on } \\
\text { County Trunk G, in Belmont, Wis. }\end{array}$ & 5.62 & $\begin{array}{l}\text { Aug. } 10,1972 \\
\text { June } 28,1973 \\
\text { Aug. } 2,1973 \\
\text { Oct. 9, } 1975 \\
\text { Sept. } 14,1976\end{array}$ & $\begin{array}{l}2.77 \\
6.17 \\
3.52 \\
1.98 \\
.98\end{array}$ & 1.2 & .74 & c \\
\hline \multirow[t]{2}{*}{ P19 } & Wolf Creek & $\begin{array}{l}\text { NW/ANE } 1 / 4 \text { sec. } 9, \text { T. } 1 \text { N., R. } 4 \text { E., } \\
\text { Lafayette County, at State Highway } \\
\text { 11, at Gratiot, Wis. }\end{array}$ & 27.6 & $\begin{array}{l}\text { Sept. } 17,1969 \\
\text { June } 1,1972 \\
\text { Aug. } 1,1972 \\
\text { Aug. } 1,1973 \\
\text { Oct. } 8,1975 \\
\text { Sept. } 14,1976\end{array}$ & $\begin{array}{l}11.1 \\
16.3 \\
12.5 \\
26.9 \\
10.7 \\
6.20\end{array}$ & 6.4 & 3.5 & c \\
\hline & Wolf Creek & $\begin{array}{l}\text { SE1/4SE1// sec. } 4, \text { T. } 1 \text { N., R. } 4 \text { E., } \\
\text { Lafayette County, at sewage-treatment } \\
\text { plant, at Gratiot, Wis. }\end{array}$ & 28.3 & & & & & \\
\hline P26 & $\begin{array}{l}\text { East Branch } \\
\text { Pecatonica River }\end{array}$ & $\begin{array}{l}\text { SE } 1 / 2 S E 1 / 4 \text { sec. } 26, \text { T. } 4 \text { N., R. } 5 \text { E., } \\
\text { Lafayette County, at State } \\
\text { Highway } 78,1.8 \text { mi south of } \\
\text { Blanchardville, Wis. }\end{array}$ & 221 & & & 62 & 46 & a \\
\hline P29 & Dodge Branch & $\begin{array}{l}\text { SE } 1 / 4 S E 1 / \% \text { sec. } 27, \text { T. } 6 \text { N., R. } 3 \text { E., } \\
\text { lowa County, at sewage-treatment } \\
\text { plant, at Dodgeville, Wis. }\end{array}$ & 1.05 & $\begin{array}{l}\text { June } 2,1972 \\
\text { Aug. } 10,1972 \\
\text { July } 31,1973 \\
\text { Sept. } 26,1974 \\
\text { Nov. } 19,1974 \\
\text { Oct. } 9,1975 \\
\text { July } 27,1976 \\
\text { Sept. } 14,1976\end{array}$ & $\begin{array}{l}2.25 \\
.09 \\
.70 \\
.43 \\
.13 \\
.05 \\
.05\end{array}$ & .02 & $<.01$ & c \\
\hline
\end{tabular}


Table 17. Pecatonica River basin station locations and low-flow characteristics-Continued.

\begin{tabular}{|c|c|c|c|c|c|c|c|c|}
\hline $\begin{array}{l}\text { Station } \\
\text { numbe }\end{array}$ & er $^{1}$ Stream & Station location & $\begin{array}{l}\text { Drainage } \\
\text { area } \\
\left(\mathrm{mi}^{2}\right)\end{array}$ & Date & $\begin{array}{l}\text { Discharge } \\
\left(\mathrm{ft}^{3} / \mathrm{s}\right)\end{array}$ & $\begin{array}{l}\mathrm{Q}_{7,2} \\
\left(\mathrm{ft}^{3} / \mathrm{s}\right)\end{array}$ & $\begin{array}{l}0_{7,10} \\
\left(\mathrm{ft}^{3} / \mathrm{s}\right)\end{array}$ & $\begin{array}{c}\text { Accuracy } \\
\text { level }\end{array}$ \\
\hline \multirow[t]{2}{*}{ P31 } & Dodge Branch & $\begin{array}{l}\text { SE } 1 / 4 N E 1 / 4 \text { sec. } 30, \text { T. } 5 \text { N., R. } 5 \text { E., } \\
\text { lowa County, at State Highway } 191 \text {, } \\
\text { at Hollandale, Wis. }\end{array}$ & 66.0 & $\begin{array}{lc}\text { June } & 2,1972 \\
\text { Aug. } 10,1972 \\
\text { July } & 31,1973 \\
\text { Oct. } 8,1975 \\
\text { July } 27,1976 \\
\text { Sept. } 15,1976\end{array}$ & $\begin{array}{c}329.3 \\
21.7 \\
54.9 \\
27.5 \\
20.3 \\
18.2\end{array}$ & 16 & 11 & b \\
\hline & Dodge Branch & $\begin{array}{l}\text { NE } 1 / 4 S W 1 / 4 \text { sec. } 29, \text { T. } 5 \text { N., R. } 5 \text { E., } \\
\text { lowa County, at sewage-treatment } \\
\text { plant, at Hollandale, Wis. }\end{array}$ & 67.0 & & & & & \\
\hline P48 & Skinner Creek & $\begin{array}{l}\text { SE } 1 / 4 \text { SE } 1 / 4 \text { sec. } 5, T .1 \text { N., R. } 6 \text { E., } \\
\text { Green County, at bridge on County } \\
\text { Trunk MM, } 0.4 \text { mi west of } \\
\text { Browntown, Wis. }\end{array}$ & 71.0 & $\begin{array}{l}\text { Oct. } 8,1975 \\
\text { July } 27,1976 \\
\text { Sept. 13, 1976 } \\
\text { June } 23,1977\end{array}$ & $\begin{array}{l}23.5 \\
19.0 \\
15.0 \\
15.1\end{array}$ & 15 & 11 & c \\
\hline P56 & Honey Creek & $\begin{array}{l}\text { SW } 1 / 4 \text { SE } 1 / 4 \text { sec. } 33, \text { T. } 2 \text { N., R. } 7 \text { E., } \\
\text { Green County, just upstream from } \\
\text { sewage-disposal plant outlet, } \\
1.7 \text { mi west of Monroe, Wis. }\end{array}$ & 4.74 & $\begin{array}{l}\text { July } 10,1968 \\
\text { Oct. } 21,1968 \\
\text { June } 1,1972 \\
\text { Aug. } 1,1972 \\
\text { Aug. } 1,1973 \\
\text { Oct. } 7,1975 \\
\text { July } 27,1976 \\
\text { Sept. 13, } 1976\end{array}$ & $\begin{array}{l}2.78 \\
2.63 \\
2.85 \\
2.76 \\
3.97 \\
1.98 \\
2.40 \\
1.92\end{array}$ & 1.9 & 1.5 & c \\
\hline P1B & $\begin{array}{l}\text { East Branch } \\
\text { Pecatonica River } \\
\text { tributary }\end{array}$ & $\begin{array}{l}\text { NE } 1 / 4 S W 1 / 4 \text { sec. } 9, \text { T. } 5 \text { N., R. } 2 \text { E., } \\
\text { lowa County, at culvert on private } \\
\text { road, } 0.6 \text { mi east of Linden, Wis. }\end{array}$ & 2.03 & $\begin{array}{l}\text { Oct. } 10,1975 \\
\text { July } 27,1976 \\
\text { Sept. } 15,1976 \\
\text { June } 23,1977\end{array}$ & $\begin{array}{l}.88 \\
.36 \\
.33 \\
.14\end{array}$ & .33 & .15 & c \\
\hline P2B & Pedler Creek & $\begin{array}{l}\text { NW } 1 / 4 \text { SW } 1 / 4 \text { sec. } 15, \text { T. } 5 \text { N., R. } 2 \text { E., } \\
\text { lowa County, at bridge on State } \\
\text { Highway } 39,1.4 \text { mi southeast of } \\
\text { Linden, Wis. }\end{array}$ & 13.6 & $\begin{array}{l}\text { Oct. } 10,1975 \\
\text { July } 27,1976 \\
\text { Sept. } 15,1976 \\
\text { June } 23,1977\end{array}$ & $\begin{array}{l}5.42 \\
2.81 \\
2.31 \\
2.20\end{array}$ & 2.9 & 1.7 & c \\
\hline P3B & $\begin{array}{l}\text { East Branch } \\
\text { Pecatonica River }\end{array}$ & $\begin{array}{l}\text { NW1/4NE1/4 sec. } 22, \text { T. } 6 \text { N., R. } 5 \text { E., } \\
\text { lowa County, at culvert on private } \\
\text { road, } 2.1 \text { mi southeast of Barneveld, } \\
\text { Wis. }\end{array}$ & 3.99 & $\begin{array}{l}\text { July } 27,1976 \\
\text { Sept. 15, } 1976 \\
\text { June } 21,1977\end{array}$ & $\begin{array}{l}1.11 \\
1.20 \\
.81\end{array}$ & .90 & .62 & c \\
\hline P4B & Ridgeway Branch & $\begin{array}{l}\text { NW } 1 / 4 \text { SE } 1 / 4 \text { sec. } 14, \text { T. } 6 \text { N., R. } 4 \text { E., } \\
\text { lowa County, } 200 \mathrm{ft} \text { upstream from } \\
\text { bridge on private road just upstream } \\
\text { from tributary, } 0.9 \text { mi southeast of } \\
\text { Ridgeway, Wis. }\end{array}$ & .74 & $\begin{array}{l}\text { Oct. 9, } 1975 \\
\text { Julv } 27 ; 1976 \\
\text { Sept. 15, } 1976\end{array}$ & $\begin{array}{l}20 \\
20 \\
20\end{array}$ & 0 & 0 & c \\
\hline
\end{tabular}

'Based on 1971 report by Wisconsin Department of Natural Resources (DNR). Sita lettered "B" was not included in 1971 DNR report.

2 Streambed is dry.

${ }^{3}$ Additional discharge measurements are available. 
Table 18. Peshtigo River basin station locations and low-flow characteristics.

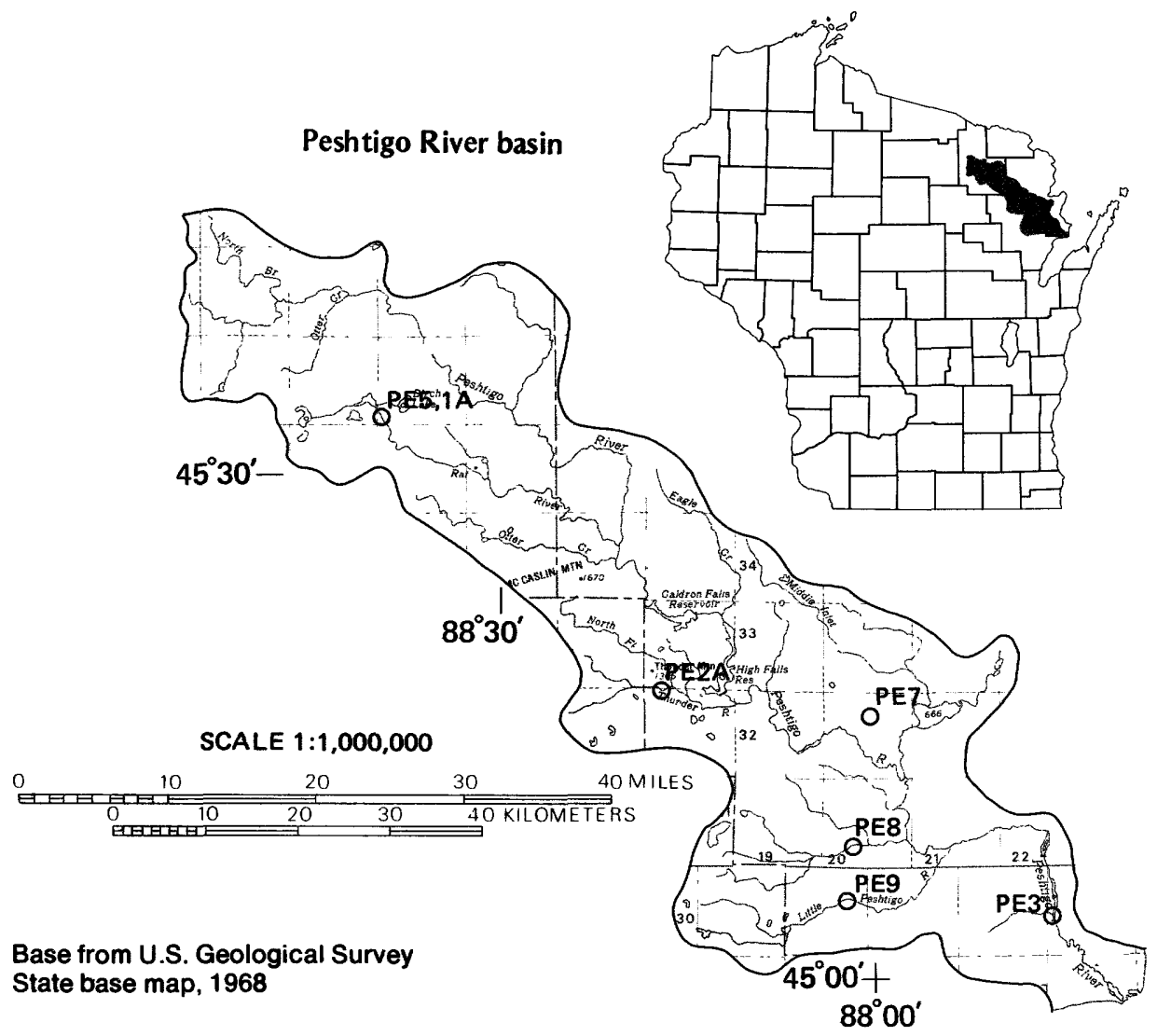

\begin{tabular}{|c|c|c|c|c|c|c|}
\hline $\begin{array}{l}\text { Station } \\
\text { number }\end{array}$ & $\underset{\text { ntreame }}{\text { Stream }}$ & Station location & $\begin{array}{l}\text { Drainage } \\
\text { area } \\
\left(\mathrm{mi}^{2}\right)\end{array}$ & Date & $\begin{array}{c}\text { Discharge } \\
\left(\mathrm{ft}^{3} / \mathrm{s}\right)\end{array}$ & $\begin{array}{c}\mathrm{Q}_{7,2} \quad \mathrm{O}_{7,10} \\
\left(\mathrm{ft}^{3} / \mathrm{s}\right)\left(\mathrm{ft}^{3} / \mathrm{s}\right)\end{array}$ Accuracy \\
\hline
\end{tabular}

PE3 Peshtigo River

PE5, Rat River

$1 \mathrm{~A}$

Rat River
NE $1 / 4 N E 1 / 4$ sec. 30, T. 30 N., R. 23 E.,

1,120

Marinette County, on left bank,

$75 \mathrm{ft}$ downstream from railroad

bridge, $0.5 \mathrm{mi}$ downstream from

WPSC powerplant in Peshtigo,

11.5 mi upstream from mouth.

SW $1 / 4$ NW $1 / 4$ sec. 31, T. 36 N., R. 15 E.,

Forest County, at culverts on

County Trunk H, at Laona, Wis.

NE $1 / 2 N W 1 / 4$ sec. 6, T. 35 N., R. 15 E., Forest County, just downstream

from confluence with Swanson Creek,

1.0 mi south of Laona, Wis.

SW $1 / 4$ SE $1 / 4$ sec. 10, T. 32 N., R. 20 E., Marinette County, at bridge on U.S.

Highway 141, 1.7 mi northeast of-

Crivitz, Wis.
$296 \quad 211$

a

33.8 Aug. 1, 1972

June 26,1973

Aug. 30, 1973

Oct. 1, 1974

July 9,1975

Aug. 4, 1975

Sept. 1, 1976

Oct. 13,1976
19.1

48.3

50.0

23.4

22.6

18.8

21.6

17.3
38.0

10.6

June 28,1973

Aug. 29, 1973

Oct. 3, 1974

July 9,1975

Aug. 7, 1975

Sept. 2, 1976
9.44

5.78

5.04

7.26

2.63

3.50 
Table 18. Peshtigo River basin station locations and low-flow characteristics-Continued.

\begin{tabular}{|c|c|c|c|c|c|c|c|c|}
\hline $\begin{array}{l}\text { Station } \\
\text { number }\end{array}$ & ${ }_{\text {name }}^{1}$ Stream & Station location & $\begin{array}{c}\text { Drainage } \\
\text { area } \\
\left(\mathrm{mi}^{2}\right)\end{array}$ & Date & $\begin{array}{c}\text { Discharge } \\
\left(\mathrm{ft}^{3} / \mathrm{s}\right)\end{array}$ & $\begin{array}{l}Q_{7,2} Q_{7} \\
\left(\mathrm{ft}^{3} / \mathrm{s}\right)\left(\mathrm{ft}^{3}\right.\end{array}$ & & $\begin{array}{c}\text { Accuracy } \\
\text { level }\end{array}$ \\
\hline \multirow[t]{2}{*}{ PE8 } & $\begin{array}{l}\text { South Branch } \\
\text { Beaver Creek }\end{array}$ & $\begin{array}{l}\text { SW\%NEY sec. } 28, \text { T. } 31 \text { N., R. } 20 \text { E., } \\
\text { Marinette County, at bridge on } \\
\text { U.S. Highway 141, } 0.5 \text { mi south } \\
\text { of Beaver, Wis. }\end{array}$ & 53.9 & $\begin{array}{l}\text { Aug. } 4,1972 \\
\text { Aug. } 28,1973 \\
\text { June } 26,1974 \\
\text { Sept. } 30,1974 \\
\text { July } 10,1975 \\
\text { Aug. } 7,1975 \\
\text { Sept. } 3,1976\end{array}$ & $\begin{array}{r}3_{24.3} \\
19.5 \\
27.6 \\
34.4 \\
41.4 \\
16.6 \\
19.3\end{array}$ & 15 & 11 & b \\
\hline & $\begin{array}{l}\text { South Branch } \\
\text { Beaver Creek } \\
\text { tributary }\end{array}$ & $\begin{array}{l}\text { NE } 1 / N W 1 / \text { sec. } 2, T .30 \text { N., R. } 20 \text { E., } \\
\text { Marinette County, at sewage- } \\
\text { treatment plant, } 0.9 \text { mi north of } \\
\text { Pound, Wis. }\end{array}$ & 2.41 & $\begin{array}{l}\text { Aug. } 4,1972 \\
\text { Aug. } 28,1973\end{array}$ & ${ }^{2} 0$ & $\mathbf{0}$ & $\mathbf{0}$ & c \\
\hline PE9 & $\begin{array}{l}\text { Little Peshtigo } \\
\text { River }\end{array}$ & $\begin{array}{l}\text { NE } 1 / \text { NW1/4 sec. } 14, T .30 \text { N., R. } 20 \text { E., } \\
\text { Marinette County, at bridge on } \\
\text { U.S. Highway } 141,0.8 \text { mi north } \\
\text { of Coleman, Wis. }\end{array}$ & 43.8 & $\begin{array}{l}\text { Aug. } 3,1972 \\
\text { June } 28,1973 \\
\text { Aug. } 28,1973 \\
\text { Sept. 30, } 1974 \\
\text { July } 9,1975 \\
\text { Aug. } 7,1975 \\
\text { Sept. } 3,1976\end{array}$ & $\begin{array}{c}9.97 \\
67.7 \\
12.5 \\
10.9 \\
12.1 \\
4.22 \\
2.12\end{array}$ & 3.6 & 2.1 & c \\
\hline PE2A & $\begin{array}{l}\text { South Fork } \\
\text { Thunder River }\end{array}$ & $\begin{array}{l}\text { SWY NWW/4 sec. } 5, \text { T. } 32 \text { N., R. } 18 \text { E.. } \\
\text { Marinette County, just upstream } \\
\text { from falls at Thunder River Fish } \\
\text { Hatchery, } 11.5 \text { mi northeast of } \\
\text { Mountain, Wis. }\end{array}$ & 20.7 & $\begin{array}{l}\text { July } 10,1975 \\
\text { Aug. } 6,1975 \\
\text { Sept. } 2,1976 \\
\text { Oct. } 13,1976 \\
\text { July } 25,1977\end{array}$ & $\begin{array}{c}11.8 \\
7.17 \\
9.58 \\
6.82 \\
10.5\end{array}$ & 7.6 & 5.8 & c \\
\hline
\end{tabular}

1 Based on 1969 report by Wisconsin Department of Natural Resources (DNR). Site lettered "A" was not included in 1969 DNR report.

${ }^{2}$ Streambed is dry.

${ }^{3}$ Additional discharge measurements are available. 
Table 19. Lower Rock River basin station locations and low-flow characteristics.

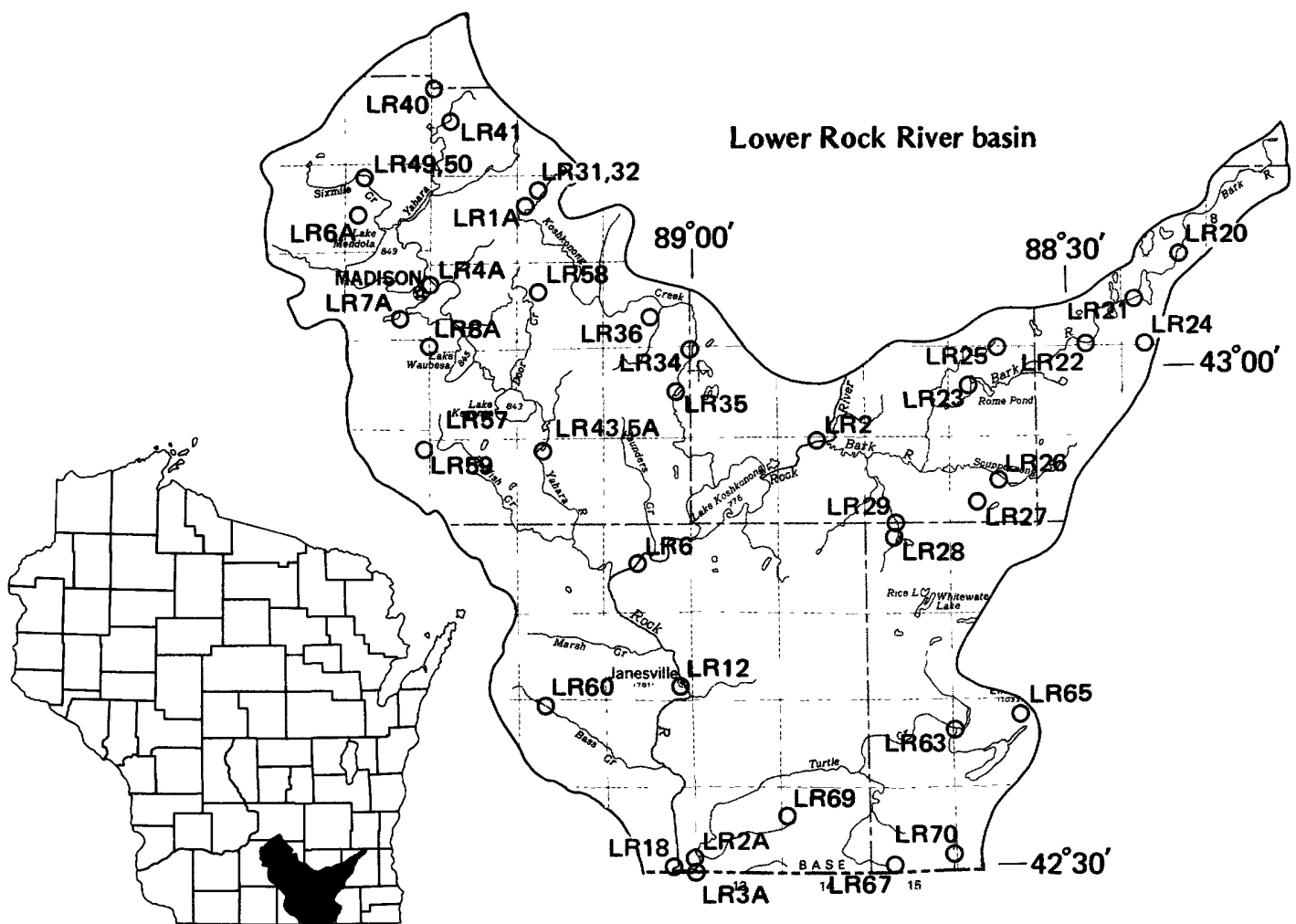

Base from U.S. Geological Survey State base map, 1968

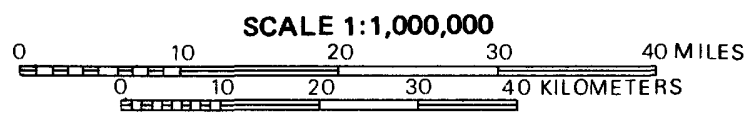

\begin{tabular}{|c|c|c|c|c|c|c|c|c|}
\hline $\begin{array}{l}\text { Station } \\
\text { number }\end{array}$ & ${ }^{1} \underset{\text { name }}{\text { Stream }}$ & Station location & $\begin{array}{l}\text { Drainage } \\
\text { area } \\
\left(\mathrm{mi}^{2}\right)\end{array}$ & Date & $\begin{array}{c}\text { Discharge } \\
\left(\mathrm{ft}^{3} / \mathrm{s}\right)\end{array}$ & $\begin{array}{l}\mathrm{O}_{7,2} \\
\left(\mathrm{ft}^{3} / \mathrm{s}\right)\end{array}$ & $\begin{array}{r}\mathrm{Q}_{7,10} \\
\left(\mathrm{ft}^{3} / \mathrm{s}\right)\end{array}$ & $\begin{array}{c}\text { Accuracy } \\
\text { level }\end{array}$ \\
\hline LR2 & Rock River & $\begin{array}{l}\text { SW1/4NW1/4 sec. } 3, \text { T. } 5 \text { N., R. } 14 \text { E., } \\
\text { Jefferson County, at U.S. } \\
\text { Highway } 12 \text {, at Fort Atkinson, Wis. }\end{array}$ & 2,190 & $\begin{array}{l}\text { Oct. } 27,1966 \\
\text { Nov. } 29,1972 \\
\text { Sept. } 24,1975 \\
\text { June } 29,1977 \\
\text { Sept. } 8,1977\end{array}$ & $\begin{array}{c}146 \\
1,115 \\
579 \\
202 \\
387\end{array}$ & 160 & 44 & c \\
\hline LR12 & Rock River & $\begin{array}{l}\text { NW1/4NE1/4 sec. 1, T. } 2 \text { N., R. } 12 \text { E., } \\
\text { Rock County, at bridge on } \\
\text { Jackson Street, at Janesville, Wis. }\end{array}$ & 3,310 & $\begin{array}{l}\text { Sept. } 30,1975 \\
\text { June } 29,1977 \\
\text { Sept. } 8,1977\end{array}$ & $\begin{array}{l}960 \\
278 \\
947\end{array}$ & $\underline{6}$ & $\underline{6}$ & \\
\hline LR18 & Rock River & $\begin{array}{l}\text { SW1/4SE } 1 / 4 \text { sec. } 35, \text { T. } 1 \text { N., R. } 12 \text { E., } \\
\text { Rock County, at sewage-treatment } \\
\text { plant, in Beloit, Wis. }\end{array}$ & 3,463 & $\begin{array}{l}\text { Sept. 22, } 1975 \\
\text { June 28, } 1977 \\
\text { Sept. 28, } 1977\end{array}$ & $\begin{array}{l}787 \\
622 \\
997\end{array}$ & $\underline{6}$ & $\underline{6}$ & \\
\hline LR20 & Bark River & $\begin{array}{l}\text { NE1/4SE } 1 / 4 \text { sec. 3, T. } 7 \text { N., R. } 18 \text { E., } \\
\text { Waukesha County, at bridge on } \\
\text { County Trunk E, } 0.7 \text { mi south } \\
\text { of Hartland, Wis. }\end{array}$ & 32.4 & $\begin{array}{l}\text { June } 2,1972 \\
\text { July } 23,1973 \\
\text { Nov. } 7,1973 \\
\text { Sept. 23, } 1975 \\
\text { Aug. } 15,1978\end{array}$ & $\begin{array}{l}18.0 \\
23.9 \\
27.6 \\
25.2 \\
19.6\end{array}$ & $\underline{7}$ & $\underline{I}$ & \\
\hline LR21 & Bark River & $\begin{array}{l}\text { SW } 1 / 4 \text { SW } 1 / 4 \text { sec. } 17, \text { T. } 7 \text { N., R. } 18 \text { E., } \\
\text { Waukesha County, at dam on outlet } \\
\text { of Nagawicka Lake, in Delafield, } \\
\text { Wis. }\end{array}$ & 44.9 & $\begin{array}{lc}\text { June } & 2,1972 \\
\text { Nov. } 29,1972 \\
\text { July } 23,1973 \\
\text { Nov. } 7,1973 \\
\text { Sept. } 23,1975 \\
\text { Aug. } 15,1978\end{array}$ & $\begin{array}{c}18.2 \\
6.89 \\
21.2 \\
91.2 \\
20.3 \\
17.2\end{array}$ & $\underline{7}$ & $\underline{7}$ & \\
\hline
\end{tabular}


Table 19. Lower Rock River basin station locations and low-flow characteristics-Continued.

\begin{tabular}{|c|c|c|c|c|c|c|c|c|}
\hline $\begin{array}{l}\text { Station } \\
\text { number }\end{array}$ & 1 name & Station location & $\begin{array}{l}\text { Drainage } \\
\text { area } \\
\left(\mathrm{mi}^{2}\right)\end{array}$ & Date & $\begin{array}{c}\text { Discharge } \\
\left(\mathrm{ft}^{3} / \mathrm{s}\right)\end{array}$ & $\begin{array}{l}\mathrm{a}_{7,2} \mathrm{a}_{7} \\
\left(\mathrm{ft}^{3} / \mathrm{s}\right)\left(\mathrm{ft}^{3}\right.\end{array}$ & $\begin{array}{l}7,10 \\
3 / s)\end{array}$ & $\underset{\text { level }}{\text { Accuracy }}$ \\
\hline LR22 & Bark River & $\begin{array}{l}\text { SE1/2SW1/4 sec. } 34, \text { T. } 7 \text { N., R. } 17 \text { E., } \\
\text { Waukesha County, at bridge on } \\
\text { County Trunk } Z \text {, } 0.4 \text { mi north } \\
\text { of Dousman, Wis. }\end{array}$ & 56.8 & $\begin{array}{l}\text { June } 2,1972 \\
\text { July } 23,1973 \\
\text { Aug. } 15,1973 \\
\text { Nov. } 7,1973 \\
\text { Sept. } 23,1975 \\
\text { Aug. } 15,1978\end{array}$ & $\begin{array}{l}19.7 \\
32.1 \\
31.4 \\
32.7 \\
28.6 \\
30.3\end{array}$ & 7 & $\underline{7}$ & \\
\hline LR23 & Bark River & $\begin{array}{l}\text { NW//SE1/4 sec. 17, T. } 6 \text { N., R. } 16 \text { E., } \\
\text { Jefferson County, at bridge on } \\
\text { State Highway } 135 \text {, at Rome, Wis. }\end{array}$ & 114 & $\begin{array}{l}\text { Nov. } 28,1972 \\
\text { June } 27,1977 \\
\text { Sept. } 8,1977 \\
\text { Aug. } 15,1978\end{array}$ & $\begin{array}{l}102 \\
13.0 \\
31.8 \\
42.8\end{array}$ & 13 & 4.1 & c \\
\hline LR24 & $\begin{array}{l}\text { Scuppernong } \\
\text { Creek }\end{array}$ & $\begin{array}{l}\text { SE } 1 / 4 S^{1} 1 / 4 \text { sec. } 32, T .7 \text { N., R. } 18 \text { E., } \\
\text { Waukesha County, at culvert on } \\
\text { private road, } 1.1 \text { mi northwest } \\
\text { of Wales, Wis. }\end{array}$ & 2.28 & $\begin{array}{l}\text { June } 2,1972 \\
\text { July } 23,1973 \\
\text { Nov. } 7,1973 \\
\text { Sept. } 23,1975\end{array}$ & $\begin{array}{l}.70 \\
1.75 \\
1.36 \\
.91\end{array}$ & .18 & .09 & c \\
\hline LR25 & Duck Creek & $\begin{array}{l}\text { SE } 1 / 4 \text { NE } 1 / 4 \text { sec. } 27, T .7 \text { N., R. } 16 \text { E., } \\
\text { Jefferson County, at culvert on } \\
\text { State Highway 135, } 1.9 \text { mi north } \\
\text { of Sullivan, Wis. }\end{array}$ & 7.44 & $\begin{array}{l}\text { June } 2,1972 \\
\text { July } 23,1973 \\
\text { Oct. } 17,1973 \\
\text { Nov. } 7,1973 \\
\text { Sept. } 23,1975\end{array}$ & $\begin{array}{l}1.24 \\
.30 \\
.40 \\
.86 \\
.26\end{array}$ & .09 & .02 & c \\
\hline LR26 & $\begin{array}{l}\text { Scuppernong } \\
\text { River }\end{array}$ & $\begin{array}{l}\text { NE1/4NE1/4 sec. } 22, \text { T. } 5 \text { N., R. } 16 \text { E., } \\
\text { Jefferson County, at bridge at } \\
\text { County Trunk E, at Palmyra } \\
\text { sewage-treatment plant. }\end{array}$ & 29.4 & $\begin{array}{l}\text { June } 2,1972 \\
\text { July } 23,1973 \\
\text { Nov. } 7,1973 \\
\text { Sept. } 23,1975\end{array}$ & $\begin{array}{l}13.2 \\
19.6 \\
22.3 \\
13.7\end{array}$ & 6.8 & 3.4 & c \\
\hline LR27 & Spring Creek & $\begin{array}{l}\text { SWY SWY sec. } 20, \text { T. } 5 \text { N., R. } 16 \text { E., } \\
\text { Jefferson County, at bridge on } \\
\text { State Highway } 59,5.6 \text { mi northeast } \\
\text { of Whitewater, Wis. }\end{array}$ & 10.1 & $\begin{array}{l}\text { Oct. } 18,1973 \\
\text { Sept. } 23,1975 \\
\text { June } 27,1977 \\
\text { Sept. } 8,1977\end{array}$ & $\begin{array}{l}15.2 \\
12.7 \\
8.47 \\
9.59\end{array}$ & 9.1 & 7.8 & c \\
\hline LR28 & Whitewater Creek & $\begin{array}{l}\text { SW1/4SE1/4 sec. } 4, T \text {, } 4 \mathrm{~N} ., \mathrm{R} .15 \mathrm{E} . \text {, } \\
\text { Walworth County, on town road, } \\
\text { at Whitewater, Wis. }\end{array}$ & 24.1 & $\begin{array}{l}\text { June } 27,1977 \\
\text { Sept. } 8,1977 \\
\text { Aug. } 16,1978\end{array}$ & $\begin{array}{l}8.05 \\
10.7 \\
19.2\end{array}$ & 10 & 7.7 & c \\
\hline LR29 & Whitewater Creek & $\begin{array}{l}\text { NW } 1 / 4 \text { NW } 1 / 4 \text { sec. } 4, \text { T. } 4 \text { N., R. } 15 \text { E., } \\
\text { Walworth County, at bridge on } \\
\text { private road on county line, } \\
0.6 \text { mi north of post office, } \\
\text { at Whitewater, Wis. }\end{array}$ & 42.7 & $\begin{array}{lr}\text { Nov. } & 2,1965 \\
\text { Sept. } 25,1971 \\
\text { June } 2,1972 \\
\text { July } 25,1973 \\
\text { Aug. } 9,1973 \\
\text { Nov. } 6,1973 \\
\text { Sept. } 17,1975 \\
\text { June } 27,1977 \\
\text { Sept. } 8,1977\end{array}$ & $\begin{array}{l}33.2 \\
14.3 \\
20.4 \\
27.4 \\
25.3 \\
34.1 \\
25.0 \\
11.5 \\
16.5\end{array}$ & 13 & 10 & c \\
\hline $\begin{array}{l}\text { LR31, } \\
\text { 32, }\end{array}$ & $\begin{array}{l}\text { Koshkonong } \\
\text { Creek }\end{array}$ & $\begin{array}{l}\text { NW } 1 / 4 \text { NE } 1 / 4 \text { sec. } 8, \text { T. } 8 \text { N., R. } 11 \text { E., } \\
\text { Dane County, at sewage-treatment } \\
\text { plant, at Sun Prairie, Wis. }\end{array}$ & 3.06 & $\begin{array}{l}\text { June } 1,1972 \\
\text { July } 23,1973 \\
\text { Nov. } 5,1973 \\
\text { Sept. 26, } 1976\end{array}$ & $\begin{array}{l}.25 \\
.54 \\
.13 \\
.09\end{array}$ & .05 & .01 & c \\
\hline LR34 & $\begin{array}{l}\text { Koshkonong } \\
\text { Creek }\end{array}$ & $\begin{array}{l}\text { NWY NEY N sec. } 12, \text { T. } 6 \text { N., R. } 12 \text { E., } \\
\text { Dane County, at bridge on U.S. } \\
\text { Highway } 12 \text {, at Cambridge, Wis. }\end{array}$ & 134 & $\begin{array}{l}\text { June } 2,1972 \\
\text { July } 24,1973 \\
\text { Nov. } 5,1973 \\
\text { Sept. } 24,1975\end{array}$ & $\begin{array}{l}23.2 \\
37.7 \\
45.1 \\
26.8\end{array}$ & 15 & 8.4 & c \\
\hline LR35 & $\begin{array}{l}\text { Koshkonong } \\
\text { Creek }\end{array}$ & $\begin{array}{l}\text { SW } 1 / 4 N W^{1} 1 / 4 \text { sec. } 24, \text { T. } 6 \text { N., R. } 12 \text { E., } \\
\text { Dane County, at sewage-treatment } \\
\text { plant, in Rockdale, just downstream } \\
\text { from dam on Rockdale Millpond. }\end{array}$ & 146 & $\begin{array}{l}\text { June } 2,1972 \\
\text { July } 24,1973 \\
\text { Nov. } 5,1973 \\
\text { Sept. } 24,1975\end{array}$ & $\begin{array}{l}27.6 \\
38.2 \\
49.0 \\
35.2\end{array}$ & 17 & 9.2 & c \\
\hline LR36 & $\begin{array}{l}\text { Mud Creek } \\
\text { tributary }\end{array}$ & $\begin{array}{l}\text { SW } 1 / 4 \text { SW } 1 / 4 \text { sec. } 22, \text { T. } 7 \text { N., R. } 12 \text { E., } \\
\text { Dane County, at sewage-treatment } \\
\text { plant, at Deerfield, Wis. }\end{array}$ & .10 & Sept. 24, 1975 & ${ }^{4} 0$ & 0 & 0 & c \\
\hline & $\begin{array}{l}\text { Mud Creek } \\
\text { tributary }\end{array}$ & $\begin{array}{l}\text { NW1/4SW1/4 sec. } 22, \text { T. } 7 \text { N., R. } 12 \text { E., } \\
\text { Dane County, } 0.2 \text { mi downstream } \\
\text { from Deerfield sewage-treatment } \\
\text { plant outfall. }\end{array}$ & .64 & $\begin{array}{lc}\text { June } & 2,1972 \\
\text { July } & 24,1973 \\
\text { Nov. } & 5,1973 \\
\text { June } & 24,1974\end{array}$ & $\begin{array}{l}.43 \\
.20 \\
.66 \\
.43\end{array}$ & $\underline{\mathbf{5}}$ & $\underline{5}$ & \\
\hline
\end{tabular}


Table 19. Lower Rock River basin station locations and low-flow characteristics-Continued.

\begin{tabular}{|c|c|c|c|c|c|c|c|c|}
\hline $\begin{array}{l}\text { Station } \\
\text { number }\end{array}$ & Stream & Station location & $\begin{array}{l}\text { Drainage } \\
\text { area } \\
\left(\mathrm{mi}^{2}\right)\end{array}$ & Date & $\begin{array}{c}\text { Discharge } \\
\left(\mathrm{ft}^{3} / \mathrm{s}\right)\end{array}$ & $\begin{array}{l}\mathrm{O}_{7,2} \mathrm{Q}_{7} \\
\left(\mathrm{ft}^{3} / \mathrm{s}\right)\left(\mathrm{ft}^{3}\right.\end{array}$ & $\begin{array}{l}7,10 \\
3 / s)\end{array}$ & $\begin{array}{c}\text { Accuracy } \\
\text { level }\end{array}$ \\
\hline LR40 & $\begin{array}{l}\text { Yahara River } \\
\text { tributary }\end{array}$ & $\begin{array}{l}\text { NWYSEY// sec. 1, T. } 9 \text { N., R. } 9 \text { E., } \\
\text { Dane County, at sewage-disposal } \\
\text { pond, } 0.9 \text { mi northwest of } \\
\text { Morrisonville, Wis. }\end{array}$ & 18.7 & 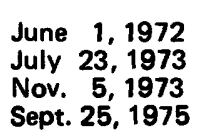 & $\begin{array}{l}2 \\
20 \\
2 \\
2 \\
2\end{array}$ & 0 & 0 & c \\
\hline LR41 & Yahara River & $\begin{array}{l}\text { NWYNWY, sec. 17, T. } 9 \text { N., R. } 10 \text { E., } \\
\text { Dane County, at bridge on County } \\
\text { Trunk CV, at DeForest, Wis. }\end{array}$ & 39.2 & $\begin{array}{l}\text { June } 1,1972 \\
\text { July } 23,1973 \\
\text { Nov. } 5,1973 \\
\text { Sept. } 25,1975\end{array}$ & $\begin{array}{c}4.03 \\
10.8 \\
13.5 \\
7.36\end{array}$ & 2.6 & 1.6 & c \\
\hline $\begin{array}{l}\text { LR43, } \\
\text { 5A }\end{array}$ & Yahara River & $\begin{array}{l}\text { SEYNEY// sec. 8, T. } 5 \text { N., R. } 11 \text { E., } \\
\text { Dane County, at sewage-treatment } \\
\text { plant, at Stoughton, Wis. }\end{array}$ & 410 & $\begin{array}{l}\text { June } 2,1972 \\
\text { July } 26,1973 \\
\text { Nov. } 8,1973 \\
\text { Sept. } 23,1975 \\
\text { Aug. } 14,1978\end{array}$ & $\begin{array}{l}120 \\
196 \\
283 \\
195 \\
170\end{array}$ & 35 & 15 & c \\
\hline$\underset{50}{\operatorname{LR} 49}$ & Sixmile Creek & $\begin{array}{l}\text { SWY NEY sec. } 8, \text { T. } 8 \text { N., R. } 9 \text { E., } \\
\text { Dane County, at sewage-treatment } \\
\text { plant, at Waunakee, Wis. }\end{array}$ & 38.3 & $\begin{array}{l}\text { June } 1,1972 \\
\text { July } 23,1973 \\
\text { Nov. } 5,1973 \\
\text { Sept. } 25,1975\end{array}$ & $\begin{array}{c}2.91 \\
9.89 \\
13.5 \\
8.01\end{array}$ & .60 & .23 & c \\
\hline LR58 & $\begin{array}{l}\text { Door Creek } \\
\text { tributary }\end{array}$ & $\begin{array}{l}\text { SWYNE1// sec. 17, T. } 7 \text { N., R. } 11 \text { E., } \\
\text { Dane County, just upstream from } \\
\text { sewage-disposal ponds, } 0.8 \mathrm{mi} \\
\text { west of Cottage Grove, Wis. }\end{array}$ & 2.73 & 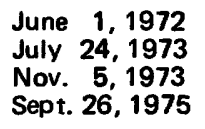 & $\begin{array}{l}.74 \\
.74 \\
1.59 \\
.63\end{array}$ & .31 & .13 & c \\
\hline LR59 & Oregon Branch & $\begin{array}{l}\text { NWYNEY\% sec. 12, T. } 5 \text { N., R. } 9 \text { E., } \\
\text { Dane County, at sewage-treatment } \\
\text { plant, at Oregon, Wis. }\end{array}$ & 11.1 & $\begin{array}{l}\text { June } 2,1972 \\
\text { July } 23,1973 \\
\text { Nov. } 8,1973 \\
\text { Sept. 26, } 1975\end{array}$ & $\begin{array}{l}<.05 \text { est. } \\
.14 \\
.17 \\
.17\end{array}$ & .01 & $<.01$ & c \\
\hline LR60 & Bass Creek & $\begin{array}{l}\text { NWYSWY sec. } 4, \text { T. } 2 \text { N., R. } 11 \text { E., } \\
\text { Rock County, at bridge on State } \\
\text { Highway } 11,0.7 \text { mi south of } \\
\text { Footville, Wis. }\end{array}$ & 14.0 & $\begin{array}{l}\text { Aug. } 14,1968 \\
\text { Oct. } 21,1968 \\
\text { June } 1,1972 \\
\text { July } 23,1973 \\
\text { Nov. } 8,1973 \\
\text { Sept. 24, } 1975 \\
\text { July } 27,1976\end{array}$ & $\begin{array}{l}5.61 \\
6.16 \\
6.95 \\
7.88 \\
8.26 \\
6.07 \\
5.42\end{array}$ & 4.2 & 3.3 & c \\
\hline LR63 & Turtle Creek & $\begin{array}{l}\text { NWY NWY/ sec. 18, T. } 2 \text { N., R. } 16 \text { E., } \\
\text { Walworth County, at Delavan sewage- } \\
\text { treatment plant. }\end{array}$ & 83.3 & $\begin{array}{lr}\text { Nov. } & 1,1965 \\
\text { June } & 2,1972 \\
\text { July } & 25,1973 \\
\text { Aug. } & 9,1973 \\
\text { Nov. } & 6,1973 \\
\text { Sept. } 22,1975\end{array}$ & $\begin{array}{l}62.3 \\
15.3 \\
25.1 \\
35.9 \\
70.8 \\
17.6\end{array}$ & 6.9 & 4.2 & c \\
\hline \multirow[t]{2}{*}{ LR65 } & Jackson Creek & $\begin{array}{l}\text { NW1/sW1/4 sec. 7, T. } 2 \text { N., R. } 17 \text { E., } \\
\text { Walworth County, at bridge on } \\
\text { State Highway } 67,1.7 \text { mi south } \\
\text { of Elkhorn, Wis. }\end{array}$ & 9.69 & $\begin{array}{lr}\text { Aug. } & 14,1968 \\
\text { Oct. } 17,1968 \\
\text { June } 2,1972 \\
\text { July } 25,1973 \\
\text { Aug. } 9,1973 \\
\text { Nov. } 6,1973 \\
\text { Sept. } 22,1975\end{array}$ & $\begin{array}{l}1.15 \\
.62 \\
.83 \\
1.26 \\
2.41 \\
2.47 \\
.67\end{array}$ & .41 & .27 & c \\
\hline & $\begin{array}{l}\text { Jackson Creek } \\
\text { tributary }\end{array}$ & $\begin{array}{l}\text { SW1/4SW1/ sec. } 6, \text { T. } 2 \text { N., R. } 17 \text { E., } \\
\text { Walworth County, at State Highway } \\
67,0.9 \text { mi south of Elkhorn, Wis. }\end{array}$ & 2.00 & & & & & \\
\hline LR67 & $\begin{array}{l}\text { Little Turtle } \\
\text { Creek }\end{array}$ & $\begin{array}{l}\text { NW } 1 / 4 S E 1 / 4 \text { sec. } 32, \text { T. } 1 \text { N., R. } 15 \text { E., } \\
\text { Walworth County, at sewage- } \\
\text { treatment plant, } 1.0 \text { mi west of } \\
\text { Sharon, Wis. }\end{array}$ & 1.83 & $\begin{array}{l}\text { June } 2,1972 \\
\text { July } 25,1973 \\
\text { Nov. } 6,1973 \\
\text { Sept. 22, } 1975\end{array}$ & $\begin{array}{l}.05 \text { est. } \\
.18 \\
.44 \\
.02\end{array}$ & $<.01$ & $<.01$ & c \\
\hline LR69 & Spring Brook & $\begin{array}{l}\text { SWYSWY\% sec. 18, T. } 1 \text { N., R. } 14 \text { E., } \\
\text { Rock County, at culvert on Clinton } \\
\text { Corners Road, } 1.7 \text { mi southwest of } \\
\text { Clinton, Wis. }\end{array}$ & 2.79 & $\begin{array}{l}\text { June } 2,1972 \\
\text { July } 25,1973 \\
\text { Nov. } 6,1973 \\
\text { Sept. 22, } 1975\end{array}$ & $\begin{array}{l}.70 \\
1.22 \\
1.46 \\
.62\end{array}$ & .30 & .20 & c \\
\hline LR70 & Piscasaw Creek & $\begin{array}{l}\text { SE } 1 / 2 \text { SE1/ sec. } 25, \text { T. } 1 \text { N., R. } 15 \text { E., } \\
\text { Walworth County, at bridge on } \\
\text { County Trunk B, } 3.3 \text { mi east of } \\
\text { Sharon, Wis. }\end{array}$ & 11.2 & $\begin{array}{l}\text { June } 2,1972 \\
\text { June } 26,1972 \\
\text { July } 25,1973 \\
\text { Nov. } 6,1973 \\
\text { Sept. 22, } 1975\end{array}$ & $\begin{array}{l}3.64 \\
3.59 \\
8.12 \\
5.06 \\
4.48\end{array}$ & 1.9 & 1.3 & c \\
\hline
\end{tabular}


Table 19. Lower Rock River basin station locations and low-flow characteristics-Continued.

\begin{tabular}{|c|c|c|c|c|c|c|c|c|}
\hline \multirow{2}{*}{$\begin{array}{l}\text { Station } \\
\text { number }\end{array}$} & \multirow{2}{*}{$\begin{array}{l}{ }_{\text {Stream }}^{\text {Same }} \\
\text { Koshkonong Creek }\end{array}$} & \multirow{2}{*}{$\begin{array}{l}\text { Station location } \\
\text { W1/4 sec. } 18, \text { T. } 8 \text { N., R. } 11 \text { E., } \\
\text { County, } 2.7 \text { mi downstream } \\
\text { ewage-treatment plant, } \\
\text { Prairie, Wis. }\end{array}$} & \multirow{2}{*}{$\begin{array}{r}\begin{array}{c}\text { Drainage } \\
\text { area } \\
\left(\mathrm{mi}^{2}\right)\end{array} \\
7.16\end{array}$} & \multirow{2}{*}{$\begin{array}{l}\text { Date } \\
\\
\text { pt. } 25,1975 \\
\text { ine } 27,1977 \\
\text { pt. } 7,1977\end{array}$} & \multirow{2}{*}{$\begin{array}{r}\begin{array}{c}\text { Discharge } \\
\left(\mathrm{ft}^{3} / \mathrm{s}\right)\end{array} \\
\\
\\
2.68 \\
2.24 \\
3.36\end{array}$} & \multicolumn{3}{|c|}{$\begin{array}{ll}0_{7,2} & 0_{7,10} \\
\left(\mathrm{ft}^{3} / \mathrm{s}\right)\left(\mathrm{ft}^{3} / \mathrm{s}\right) & \begin{array}{c}\text { Accuracy } \\
\text { level }\end{array}\end{array}$} \\
\hline & & & & & & 2.0 & 1.1 & c \\
\hline LR2A & Turtle Creek & $\begin{array}{l}\text { SW1/4NW1/4 sec. } 30, \text { T. } 1 \text { N., R. } 13 \text { E., } \\
\text { Rock County, } 0.9 \text { mi upstream } \\
\text { from White Avenue Road, at } \\
\text { Beloit, Wis. }\end{array}$ & 233 & $\begin{array}{l}\text { Sept. } 22,1975 \\
\text { June } 28,1977 \\
\text { Sept. } 8,1977 \\
\text { Aug. } 14,1978\end{array}$ & $\begin{array}{l}91.5 \\
27.9 \\
34.6 \\
91.0\end{array}$ & 33 & 23 & c \\
\hline LR3A & Spring Brook & $\begin{array}{l}\text { NE } 1 / 4 \text { NW } 1 / 4 \text { sac. } 32, \text { T. } 1 \text { N., R. } 13 \text { E., } \\
\text { Rock County, at private road bridge, } \\
2.6 \text { mi northeast of post office in } \\
\text { Beloit, Wis. }\end{array}$ & 9.66 & $\begin{array}{l}\text { Sept. } 22,1975 \\
\text { June } 28,1977 \\
\text { Sept. } 8,1977 \\
\text { Aug. } 14,1978\end{array}$ & $\begin{array}{l}2.25 \\
.28 \\
.08 \\
2.22\end{array}$ & .12 & .04 & c \\
\hline LR4A & Yahara River & $\begin{array}{l}\text { NW1/4 NW1/4 sec. } 7, \text { T. } 7 \text { N., R. } 10 \text { E., } \\
\text { Dane County, at Main Street bridge, } \\
1.5 \text { mi northeast of capitol, at } \\
\text { Madison, Wis. }\end{array}$ & 235 & $\begin{array}{l}\text { Sept. } 14,1975 \\
\text { Oct. } 19,1975 \\
\text { Nov. } 15,1975 \\
\text { Dec. } 14,1975\end{array}$ & $\begin{array}{l}3116 \\
12.7 \\
68.4 \\
225\end{array}$ & $\underline{6}$ & $\underline{6}$ & \\
\hline LR6A & Spring Creek & $\begin{array}{l}\text { NE } 1 / 4 \text { NW } 1 / 4 \text { sec. } 30, \text { T. } 8 \text { N., R. } 9 \text { E., } \\
\text { Dane County, at culvert at County } \\
\text { Trunk } Q, 3.4 \text { mi southwest of } \\
\text { Waunakee, Wis. }\end{array}$ & 9.95 & $\begin{array}{l}\text { Sept. } 25,1975 \\
\text { June } 27,1977 \\
\text { Sept. } 7,1977 \\
\text { Aug. } 14,1978\end{array}$ & $\begin{array}{l}3.80 \\
.76 \\
1.54 \\
1.90\end{array}$ & .75 & .48 & c \\
\hline LR7A & Murphy Creek & $\begin{array}{l}\text { SW1/4SE } 1 / 4 \text { sec. } 26, \text { T. } 7 \text { N., R. } 9 \text { E., } \\
\text { Dane County, at Beld Street, } \\
\text { in Madison, Wis. }\end{array}$ & 8.03 & & & 0 & 0 & $\mathbf{a}$ \\
\hline LR8A & $\begin{array}{l}\text { Nine Springs } \\
\text { Creek tributary }\end{array}$ & $\begin{array}{l}\text { NW1/4NE1/4 sec. 10, T. } 6 \text { N., R. } 9 \text { E., } \\
\text { Dane County, at outiat from } \\
\text { Nevin fish hatchery, } 4.4 \mathrm{mi} \\
\text { southwest of capitol, } \\
\text { at Madison, Wis. }\end{array}$ & .12 & $\begin{array}{l}\text { Oct. } 23,1972 \\
\text { Jan. } 18,1974 \\
\text { July } 19,1974 \\
\text { Feb. } 28,1975 \\
\text { June } 26,1975 \\
\text { Sept. } 2,1975 \\
\text { Oct. } 15,1975\end{array}$ & $\begin{array}{l}32.87 \\
2.42 \\
2.66 \\
1.92 \\
2.39 \\
2.13 \\
2.43\end{array}$ & 1.5 & 1.1 & c \\
\hline
\end{tabular}

1 Based on 1971 report by Wisconsin Department of Natural Resources (DNR). Site lettered "A" was not included in 1971 DNR report.

${ }^{2}$ Streambed is dry.

${ }^{3}$ Additional discharge measurements are available.

5 No estimate possible--discharge is primarily effluent.

${ }^{6}$ No estimate possible due to regulation upstream.

7 Unable to define relationship. 
Table 20. Upper Rock River basin station locations and low-flow characteristics.

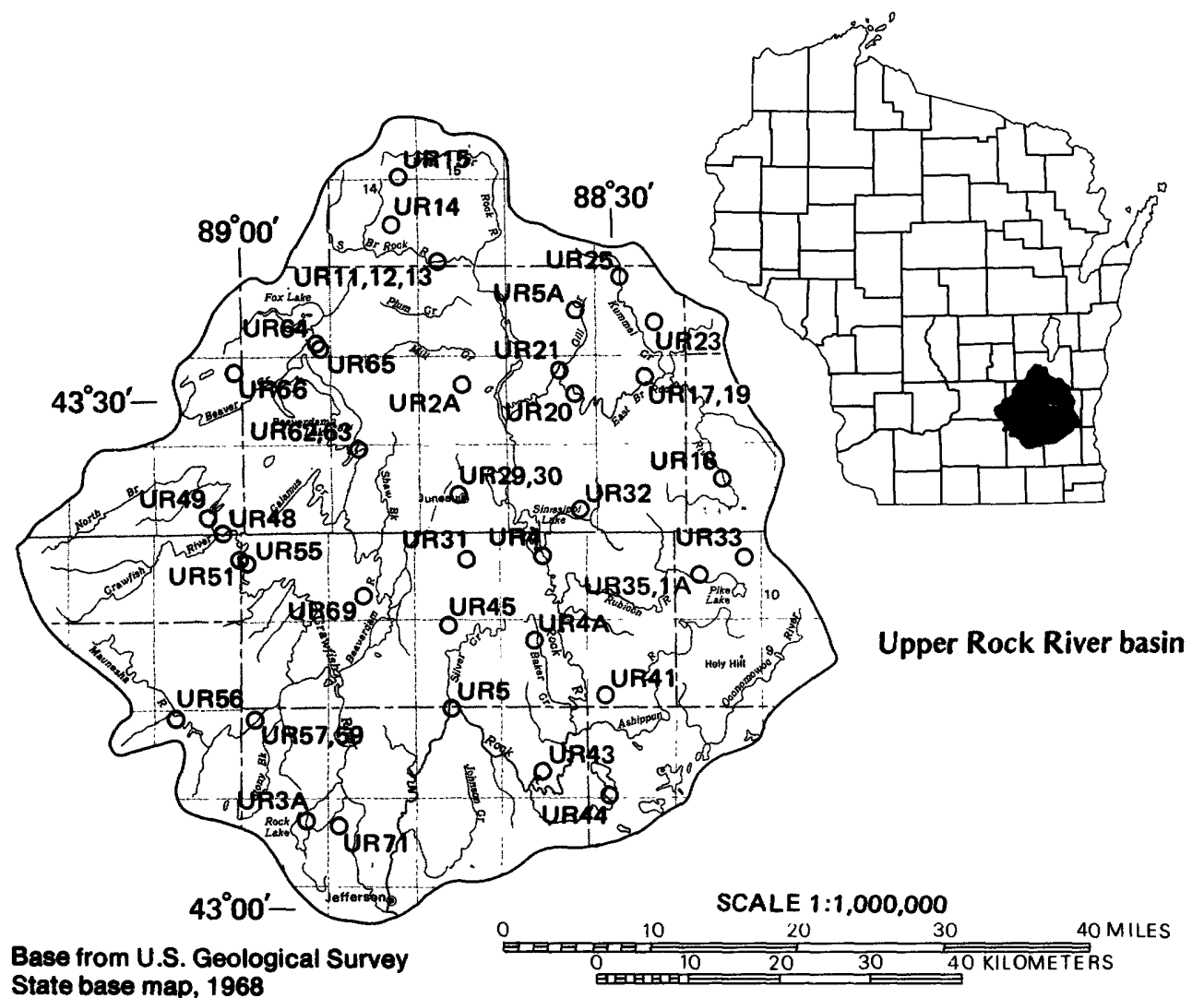

\begin{tabular}{|c|c|c|c|c|c|c|c|c|}
\hline $\begin{array}{l}\text { Station }{ }^{1} \\
\text { number }\end{array}$ & $1 \underset{\text { Stream }}{\text { name }}$ & Station location & $\begin{array}{c}\text { Drainage } \\
\text { area } \\
\left(\mathrm{mi}^{2}\right)\end{array}$ & Date & $\begin{array}{c}\text { Discharge } \\
\left(\mathrm{ft}^{3} / \mathrm{s}\right)\end{array}$ & $\begin{array}{l}\mathrm{Q}_{7,2} \mathrm{Q}_{7} \\
\left(\mathrm{ft}^{3} / \mathrm{s}\right)(\mathrm{ft}\end{array}$ & $3 / 51$ & $\begin{array}{c}\text { Accuracy } \\
\text { level }\end{array}$ \\
\hline \multirow[t]{2}{*}{ UR4 } & Rock River & $\begin{array}{l}\text { NW } 1 / 4 \text { SW } 1 / 4 \text { sec. } 10, T .10 \text { N., R. } 16 \text { E., } \\
\text { Dodge County, } 1,000 \text { ft downstream } \\
\text { from dam, downstream from Wildcat } \\
\text { Creek, at Hustisford, Wis. }\end{array}$ & 554 & $\begin{array}{l}\text { Aug. } 15,1967 \\
\text { Sept. } 10,1972 \\
\text { July } 8,1973 \\
\text { Sept. } 13,1973 \\
\text { Sept. 25, } 1975 \\
\text { July } 22,1976 \\
\text { Nov. } 12,1976\end{array}$ & $\begin{array}{c}1.45 \\
88.4 \\
142 \\
1.61 \\
3.19 \\
6.65 \\
.91\end{array}$ & $\underline{6}$ & $\underline{6}$ & \\
\hline & Rock River & $\begin{array}{l}\text { NW1/4NW1/4 sec. } 15, \text { T. } 10 \text { N., R. } 16 \text { E., } \\
\text { Dodge County, at Hustisford sewage- } \\
\text { treatment plant, } 0.6 \text { mi southeast } \\
\text { of Hustisford, Wis. }\end{array}$ & 554 & & & & & \\
\hline UR5 & Rock River & $\begin{array}{l}\text { NE } 1 / 4 \text { SW } 1 / 4 \text { sec. } 4, \text { T. } 8 \text { N., R. } 15 \text { E., } \\
\text { Jefferson County, } 700 \mathrm{ft} \\
\text { downstream from Milwaukee Street } \\
\text { bridge, at Watertown, Wis. }\end{array}$ & 969 & & & 26 & 5.3 & a \\
\hline $\begin{array}{l}\text { UR 13, } \\
11, \\
12\end{array}$ & $\begin{array}{l}\text { South Branch } \\
\text { Rock River }\end{array}$ & $\begin{array}{l}\text { SW1/4NW1/4 sec. } 33, \text { T. } 14 \mathrm{~N} ., \text { R. } 15 \mathrm{E} . \text {, } \\
\text { Fond du Lac County, } 100 \mathrm{ft} \\
\text { upstream from U.S. Highway } 151 \text {, } \\
0.8 \text { mi northeast of Waupun, Wis. }\end{array}$ & 68.6 & $\begin{array}{l}\text { June } 1,1972 \\
\text { July } 17,1973 \\
\text { Sept. 11, } 1973 \\
\text { Sept. 24, } 1975 \\
\text { July } 21,1976 \\
\text { Nov. 10, } 1976 \\
\text { July } 11,1977\end{array}$ & $\begin{array}{c}312.2 \\
12.2 \\
3.99 \\
3.30 \\
3.06 \\
.70 \\
.44\end{array}$ & .60 & .06 & a \\
\hline UR14 & $\begin{array}{l}\text { South Branch } \\
\text { Rock River } \\
\text { tributary }\end{array}$ & $\begin{array}{l}\text { SE } / / S E 1 / 4 \text { sec. } 23, T .14 \text { N., R. } 14 \text { E., } \\
\text { Fond du Lac County, at culvert on } \\
\text { County Trunk E, } 1.1 \text { mi southeast } \\
\text { of Alto, Wis. }\end{array}$ & 1.12 & $\begin{array}{l}\text { Sept. } 24,1975 \\
\text { July } 21,1976 \\
\text { Nov. 10, } 1976 \\
\text { July } 11,1977\end{array}$ & $\begin{array}{r}.56 \\
.32 \\
.36 \\
<.01\end{array}$ & $<.01$ & $<.01$ & c \\
\hline
\end{tabular}


Table 20. Upper Rock River basin station locations and low-flow characteristics-Continued.

\begin{tabular}{|c|c|c|c|c|c|c|c|c|}
\hline $\begin{array}{l}\text { Station } \\
\text { number }\end{array}$ & ${ }_{\text {Stream }}^{1}$ & Station location & $\begin{array}{l}\text { Drainage } \\
\text { area } \\
\left(\mathrm{mi}^{2}\right)\end{array}$ & Date & $\begin{array}{c}\text { Discharge } \\
\left(\mathrm{ft}^{3} / \mathrm{s}\right)\end{array}$ & $\begin{array}{l}Q_{7,2} Q \\
\left(f t^{3} / s\right)(f\end{array}$ & $\mathrm{ft}^{2}, 10$ & $\underset{\text { level }}{\text { Accuracy }}$ \\
\hline UR15 & $\begin{array}{l}\text { South Branch } \\
\text { Rock River } \\
\text { tributary }\end{array}$ & $\begin{array}{l}\text { SW1/4SW1/4 sec. } 36, \text { T. } 15 \text { N., R. } 14 \text { E., } \\
\text { Fond du Lac County, at bridge on } \\
\text { County Trunk JJ, } 1.0 \text { mi south of } \\
\text { Brandon, Wis. }\end{array}$ & 1.23 & $\begin{array}{l}\text { June } 1,1972 \\
\text { July } 17,1973 \\
\text { Sept. } 11,1973 \\
\text { Sept. } 24,1975 \\
\text { July } 21,1976\end{array}$ & $\begin{array}{l}.73 \\
.32 \\
.13 \\
.20 \\
.30\end{array}$ & 0.01 & $<0.01$ & c \\
\hline UR16 & $\begin{array}{l}\text { East Branch } \\
\text { Rock River }\end{array}$ & $\begin{array}{l}\text { SW } 1 / 4 \text { SW } 1 / 4 \text { sec. } 15, T .11 \text { N., R. } 18 \text { E., } \\
\text { Washington County, at County } \\
\text { Trunk W, } 0.3 \text { mi south of Allenton, } \\
\text { Wis. }\end{array}$ & 28.1 & $\begin{array}{l}\text { Aug. } 15,1967 \\
\text { June } 1,1972 \\
\text { July } 19,1973 \\
\text { Sept. } 12,1973 \\
\text { Sept. } 26,1975 \\
\text { July } 22,1976 \\
\text { Nov. } 12,1976\end{array}$ & $\begin{array}{l}2.95 \\
4.63 \\
5.17 \\
7.44 \\
7.36 \\
5.05 \\
5.99\end{array}$ & 2.3 & 1.3 & c \\
\hline $\begin{array}{l}\text { UR17. } \\
19\end{array}$ & $\begin{array}{l}\text { East Branch } \\
\text { Rock River }\end{array}$ & $\begin{array}{l}\text { NE } 1 / 4 \text { SW } 1 / 4 \text { sec. } 10, T .12 \text { N., R. } 17 \text { E., } \\
\text { Dodge County, just downstream } \\
\text { from dam, at Theresa, Wis. }\end{array}$ & 140 & $\begin{array}{l}\text { June } 1,1972 \\
\text { July } 18,1973 \\
\text { Sept. 12, 1973 } \\
\text { Sept. 26, } 1975 \\
\text { July 22, } 1976 \\
\text { Nov. 11, } 1976\end{array}$ & $\begin{array}{c}37.3 \\
36.7 \\
29.5 \\
3.84 \\
17.4 \\
5.50\end{array}$ & $\underline{6}$ & $\underline{6}$ & \\
\hline $\begin{array}{c}\text { UR20, } \\
21\end{array}$ & $\begin{array}{l}\text { East Branch } \\
\text { Rock River }\end{array}$ & $\begin{array}{l}\text { NE } 1 / 4 \mathrm{SE}^{1} / 4 \text { sec. } 14, \mathrm{~T} .12 \mathrm{~N} ., \mathrm{R} .16 \mathrm{E} . \text {, } \\
\text { Dodge County, at bridge on County } \\
\text { Trunk V, at Mayvilie, Wis. }\end{array}$ & 173 & $\begin{array}{l}\text { Sept. 26, } 1975 \\
\text { July 22, } 1976 \\
\text { Nov. 11, } 1976 \\
\text { July } 11,1977\end{array}$ & $\begin{array}{c}6.49 \\
14.9 \\
3.32 \\
9.04\end{array}$ & $\underline{6}$ & $\underline{6}$ & \\
\hline & $\begin{array}{l}\text { East Branch } \\
\text { Rock River }\end{array}$ & $\begin{array}{l}\text { SW1/4NE } 1 / 4 \text { sec. } 14, T .12 \text { N., R. } 16 \text { E., } \\
\text { Dodge County, at sewage-treatment } \\
\text { plant, at Mayville, Wis. }\end{array}$ & 173 & & & & & \\
\hline & $\begin{array}{l}\text { East Branch } \\
\text { Rock River }\end{array}$ & $\begin{array}{l}\text { NW } 1 / 4 \text { NE } 1 / 4 \text { sec. } 10, T .12 \mathrm{~N} ., \text { R. } 16 \mathrm{E} ., \\
\text { Dodge County, } 500 \mathrm{ft} \text { downstream } \\
\text { from Kekoskee dam, } 2.0 \mathrm{mi} \\
\text { northwest of Mayville, Wis. }\end{array}$ & 181 & & & 5.8 & 1.6 & a \\
\hline UR23 & $\begin{array}{l}\text { East Branch } \\
\text { Rock River } \\
\text { tributary }\end{array}$ & $\begin{array}{l}\text { SE1/4NE1/4 sec. } 27, T .13 \mathrm{~N} ., \mathrm{R} .17 \mathrm{E} \text {., } \\
\text { Dodge County, at culvert on country } \\
\text { road, } 1.5 \text { mi south of Lomira, Wis. }\end{array}$ & 3.70 & $\begin{array}{l}\text { June } 1,1972 \\
\text { July } 18,1973 \\
\text { Sept. 12, } 1973 \\
\text { Sept. 26, } 1975 \\
\text { July } 22,1976 \\
\text { Nov. 11, } 1976\end{array}$ & $\begin{array}{l}1.56 \\
1.87 \\
1.10 \\
.80 \\
.69 \\
.57\end{array}$ & .42 & .22 & c \\
\hline UR25 & Kummel Creek & $\begin{array}{l}\text { SW1/4NE1/4 sec. 8, T. } 13 \mathrm{~N} ., \text { R. } 17 \mathrm{E} ., \\
\text { Dodge County, just upstream from } \\
\text { sewege-treatment plant, at } \\
\text { Brownsville, Wis. }\end{array}$ & 13.1 & $\begin{array}{l}\text { June } 1,1972 \\
\text { July } 18,1973 \\
\text { Sept. 12, } 1973 \\
\text { Sept. 26, } 1975 \\
\text { July 22, } 1976\end{array}$ & $\begin{array}{l}2.08 \\
5.21 \\
1.29 \\
.59 \\
1.11\end{array}$ & .13 & .02 & c \\
\hline UR29 & Dead Creek & $\begin{array}{l}\text { NW } 1 / 4 N W 1 / 4 \text { sec. } 27, T .11 \text { N., R. } 15 \text { E., } \\
\text { Dodge County, at Juneau sewage- } \\
\text { treatment plant at south edge of } \\
\text { city, about } 0.3 \text { mi south of } \\
\text { State Highway } 115 \text { bridge. }\end{array}$ & .58 & $\begin{array}{l}\text { June } 1,1972 \\
\text { July } 18,1973 \\
\text { Sept. 13, } 1975 \\
\text { Sept. } 24,1975 \\
\text { July } 23,1976 \\
\text { Nov. } 9,1976\end{array}$ & $\begin{array}{l}.70 \\
1.36 \\
.89 \\
.96 \\
.95 \\
1.27\end{array}$ & $\underline{5}$ & $\underline{5}$ & \\
\hline UR30 & Dead Creek & $\begin{array}{l}\text { SW } 1 / \text { SW } 1 / 4 \text { sec. } 22, T .11 \text { N., R. } 15 \text { E., } \\
\text { Dodge County, just upstream from } \\
\text { Milbrew, Inc., at Juneau, Wis. }\end{array}$ & .46 & $\begin{array}{l}\text { Sept. } 24,1975 \\
\text { July } 23,1976 \\
\text { Nov. } 9,1976\end{array}$ & $\begin{array}{l}2 \\
2_{0}^{0} \\
2_{0}^{0}\end{array}$ & 0 & 0 & c \\
\hline \multirow[t]{2}{*}{ UR31 } & $\begin{array}{l}\text { Dead Creek } \\
\text { tributary }\end{array}$ & $\begin{array}{l}\text { NE } 1 / 1 \text { SW } 1 / 4 \text { sec. } 15, T .10 \text { N., R. } 15 \text { E., } \\
\text { Dodge County, at sewage-disposal } \\
\text { ponds, } 1.5 \text { mi northeast of Clyman, } \\
\text { Wis. }\end{array}$ & 4.23 & & & & & \\
\hline & $\begin{array}{l}\text { Dead Creek } \\
\text { tributary }\end{array}$ & $\begin{array}{l}\text { NW } 1 / 4 N^{N} 1 / 4 \text { sec. } 15, T .10 \text { N., R. } 15 \text { E., } \\
\text { Dodge County, at culvert on } \\
\text { State Highway } 60,2.2 \text { mi northeast } \\
\text { of Clyman, Wis. }\end{array}$ & 5.30 & $\begin{array}{l}\text { July } 18,1973 \\
\text { Sept. 13, 1973 } \\
\text { Sept. 25, } 1975 \\
\text { July } 22,1976 \\
\text { Nov. } 9,1976\end{array}$ & $\begin{array}{l}2.14 \\
2.04 \\
2.0 \\
20\end{array}$ & 0 & 0 & c \\
\hline
\end{tabular}


Table 20. Upper Rock River basin station locations and low-flow characteristics-Continued.

\begin{tabular}{|c|c|c|c|c|c|c|c|c|}
\hline $\begin{array}{l}\text { Station } \\
\text { numbe }\end{array}$ & $\mathrm{n}^{1} \underset{\text { er }}{\text { Streame }}$ & Station location & $\begin{array}{l}\text { Drainage } \\
\text { area } \\
\left(\mathrm{mi}^{2}\right)\end{array}$ & Date & $\begin{array}{c}\text { Discharge } \\
\left(\mathrm{ft}^{3} / \mathrm{s}\right)\end{array}$ & $\begin{array}{l}0_{7,2} \\
\left(\mathrm{ft}^{3} / \mathrm{s}\right)(\mathrm{f}\end{array}$ & $\left.\mathrm{ft}^{3} / \mathrm{s}\right)$ & $\begin{array}{c}\text { Accuracy } \\
\text { level }\end{array}$ \\
\hline UR32 & Wildcat Creek & $\begin{array}{l}\text { SE1/4SE } 1 / 4 \text { sec. } 26, T .11 \text { N., R. } 16 \text { E., } \\
\text { Dodge County, at town road bridge } \\
\text { about } 1.1 \text { mi southwest of Iron } \\
\text { Ridge, Wis. }\end{array}$ & 21.0 & $\begin{array}{l}\text { June } 1,1972 \\
\text { July } 18,1973 \\
\text { Sept. 12, } 1973 \\
\text { Sept. 25, } 1975 \\
\text { July } 22,1976 \\
\text { Nov. } 12,1976\end{array}$ & $\begin{array}{l}3.89 \\
1.39 \\
.85 \\
1.68 \\
.63 \\
.37\end{array}$ & 0.21 & 0.03 & c \\
\hline \multirow[t]{2}{*}{ UR33 } & Rubicon River & $\begin{array}{l}\text { NW } 1 / 4 \text { SE } 1 / 4 \text { sec. } 13, T .10 \text { N., R. } 18 \text { E., } \\
\text { Washington County, at culvert on } \\
\text { Hartford Road, } 1.1 \text { mi west of } \\
\text { Slinger, Wis. }\end{array}$ & 5.24 & $\begin{array}{lr}\text { June } & 1,1972 \\
\text { July } & 19,1973 \\
\text { Aug. } 15,1973 \\
\text { Sept. 26, } 1975 \\
\text { July } 22,1976 \\
\text { Nov. } 12,1976\end{array}$ & $\begin{array}{l}1.06 \\
.60 \\
.37 \\
.44 \\
.18 \\
.14\end{array}$ & .08 & .01 & c \\
\hline & Rubicon River & $\begin{array}{l}\text { SEE } 1 / 4 \text { SW } 1 / 4 \text { sec. } 13, T .10 \mathrm{~N} ., \text { R. } 18 \mathrm{E} \text {., } \\
\text { Washington County, just downstream } \\
\text { from confluence with intermittent } \\
\text { tributary from sewage-disposel } \\
\text { plant, } 1.3 \text { mi west of Slinger, Wis. }\end{array}$ & 6.21 & & & & & \\
\hline $\begin{array}{l}\text { UR35, } \\
\text { IA }\end{array}$ & Rubicon River & $\begin{array}{l}\text { SE1/4NW1/4 sec. } 20, T .10 \text { N., R. } 18 \text { E., } \\
\text { Washington County, at sewage- } \\
\text { treatment plant outfall, at west } \\
\text { city limits of Hartford, Wis. }\end{array}$ & 27.8 & $\begin{array}{l}\text { June } 1,1972 \\
\text { Oct. } 4,1972 \\
\text { July } 19,1973 \\
\text { Aug. } 15,1973 \\
\text { Sept. } 26,1975 \\
\text { July } 22,1976 \\
\text { Nov. } 12,1976\end{array}$ & $\begin{array}{l}4.16 \\
8.68 \\
3.11 \\
3.08 \\
2.20 \\
2.84 \\
6.22\end{array}$ & 1.8 & 1.1 & c \\
\hline UR41 & Davy Creek & $\begin{array}{l}\text { NE } 1 / 4 \text { NE1/4 sec. } 31 \text {, T. } 9 \text { N., R. } 17 \text { E., } \\
\text { Dodge County, at culvert on } \\
\text { Lincoln Road, at Ashippun, Wis. }\end{array}$ & 5.79 & $\begin{array}{l}\text { July } 21,1976 \\
\text { Oct. } 4,1976 \\
\text { July } 12,1977\end{array}$ & $\begin{array}{l}{ }_{4}^{4} \\
{ }^{4} \\
0\end{array}$ & 0 & 0 & c \\
\hline UR43 & $\begin{array}{l}\text { Tributary to } \\
\text { Rock River } \\
\text { tributary }\end{array}$ & $\begin{array}{l}\text { SW } 1 / 4 \text { SW1/4 sec. } 22, \text { T. } 8 \text { N., R. } 16 \text { E., } \\
\text { Jefferson County, at sewage- } \\
\text { treatment plant, in Ixonia, Wis. }\end{array}$ & .75 & $\begin{array}{l}\text { Nov. } 7,1973 \\
\text { Sept. } 29,1975 \\
\text { July } 21,1976 \\
\text { Oct. } 4,1976\end{array}$ & $\begin{array}{l}.03 \\
2_{0}^{.02} \\
2_{0}^{0}\end{array}$ & 0 & 0 & c \\
\hline \multirow[t]{2}{*}{ UR44 } & $\begin{array}{l}\text { Oconomowoc } \\
\text { River }\end{array}$ & $\begin{array}{l}\text { NE } 1 / 4 N W 1 / 4 \text { sec. } 5, \text { T. } 7 \text { N., R. } 17 \text { E., } \\
\text { Waukesha County, at bridge on } \\
\text { County Trunk BB, just south of } \\
\text { Oconomowoc, Wis. }\end{array}$ & 100 & $\begin{array}{l}\text { June } 2,1972 \\
\text { Sept. } 10,1972 \\
\text { July } 19,1973 \\
\text { Aug. } 15,1973 \\
\text { Sept. } 26,1975 \\
\text { July } 21,1976 \\
\text { Oct. 4, } 1976\end{array}$ & $\begin{array}{l}5.60 \\
75.0 \\
11.5 \\
29.8 \\
35.7 \\
11.7 \\
6.10\end{array}$ & 1.2 & .18 & c \\
\hline & $\begin{array}{l}\text { Oconomowoc } \\
\text { River }\end{array}$ & $\begin{array}{l}\text { SW1/4NE } 1 / 4 \text { sec. } 5, \text { T. } 7 \text { N., R. } 17 \text { E., } \\
\text { Waukesha County, } 1,400 \text { ft down- } \\
\text { stream from County Trunk BB, at } \\
\text { sewage-treatment plant outfall. }\end{array}$ & 101 & & & & & \\
\hline UR45 & Clyman Creek & $\begin{array}{l}\text { SW } 1 / 4 \mathrm{SE}^{1} / 4 \text { sec. } 4, \mathrm{~T} .9 \mathrm{~N} ., \mathrm{R} .15 \mathrm{E} . \text {, } \\
\text { Dodge County, at culvert on County } \\
\text { Trunk JM, } 3.0 \text { mi south of } \\
\text { Clyman, Wis. }\end{array}$ & 2.38 & $\begin{array}{l}\text { Sept. 25, } 1975 \\
\text { July 22, } 1976 \\
\text { Nov. 9, } 1976 \\
\text { July } 12,1977\end{array}$ & $\begin{array}{l}1.08 \\
.08 \\
.08 \\
.02\end{array}$ & .02 & $<.01$ & c \\
\hline UR48 & Crawfish River & $\begin{array}{l}\text { SW1/4 NW1/4 sec. } 2, T .10 \mathrm{~N} ., \mathrm{R} .12 \mathrm{E} . \text {, } \\
\text { Columbia County, at bridge on } \\
\text { U.S. Highway } 16,1.6 \text { mi south } \\
\text { of Fall River, Wis. }\end{array}$ & 54.2 & $\begin{array}{l}\text { Aug. } 15,1967 \\
\text { June } 2,1972 \\
\text { Sept. } 10,1972 \\
\text { July } 17,1973 \\
\text { Sept. } 11,1973 \\
\text { Sept. } 23,1975 \\
\text { July } 23,1976 \\
\text { Nov. } 9,1976\end{array}$ & $\begin{array}{l}6.76 \\
14.2 \\
17.2 \\
20.9 \\
19.6 \\
13.3 \\
10.3 \\
7.69\end{array}$ & 4.3 & 1.2 & c \\
\hline
\end{tabular}


Table 20. Upper Rock River basin station locations and low-flow characteristics-Continued.

\begin{tabular}{|c|c|c|c|c|c|c|c|c|}
\hline $\begin{array}{l}\text { Station } \\
\text { number }\end{array}$ & $1 \underset{\text { name }}{\text { Stream }}$ & Station location & $\begin{array}{l}\text { Drainage } \\
\text { area } \\
\left(\mathrm{mi}^{2}\right)\end{array}$ & Date & $\begin{array}{l}\text { Discharge } \\
\left(\mathrm{ft}^{3} / \mathrm{s}\right)\end{array}$ & $\begin{array}{l}\mathrm{Q}_{7,2} \mathrm{Q}_{7} \\
\left(\mathrm{ft}^{3} / \mathrm{s}\right)\left(\mathrm{ft}^{3}\right.\end{array}$ & $3 / 5)$ & $\begin{array}{c}\text { Accuracy } \\
\text { level }\end{array}$ \\
\hline \multirow[t]{2}{*}{ UR49 } & $\begin{array}{l}\text { North Branch } \\
\text { Crawfish River }\end{array}$ & $\begin{array}{l}\text { SE } 1 / 4 S^{1} 1 / 4 \text { sec. } 27, T .11 \text { N., R. } 12 \text { E., } \\
\text { Columbia County, at County Trunk } \\
\text { D, at Fall River, Wis. }\end{array}$ & 76.2 & $\begin{array}{l}\text { June } 2,1972 \\
\text { Sept. 10, } 1972 \\
\text { July } 17,1973 \\
\text { Sept. 11, } 1973 \\
\text { Sept. 23, } 1975 \\
\text { July } 23,1976 \\
\text { Nov. } 9,1976\end{array}$ & $\begin{array}{c}8.34 \\
19.5 \\
14.4 \\
12.1 \\
2.82 \\
1.35 \\
2.59\end{array}$ & 0.40 & 0.02 & c \\
\hline & $\begin{array}{l}\text { North Branch } \\
\text { Crawfish River }\end{array}$ & $\begin{array}{l}\text { SE1/4NE } 1 / 4 \text { sec. } 34, T .11 \text { N., R. } 12 \text { E., } \\
\text { Columbia County, at sewage- } \\
\text { treatment plant, } 0.4 \text { mi south of } \\
\text { Fall River, Wis. }\end{array}$ & 77.7 & & & & & \\
\hline \multirow[t]{2}{*}{ UR51 } & Crawfish River & $\begin{array}{l}\text { NE } 1 / 4 \text { SE } 1 / 4 \text { sec. } 13, T .10 \mathrm{~N} ., \mathrm{R} .12 \mathrm{E} . \text {., } \\
\text { Columbia County, at bridge on } \\
\text { U.S. Highway } 16,0.3 \text { mi southeast } \\
\text { of Columbus, Wis. }\end{array}$ & 164 & $\begin{array}{l}\text { June } 2,1972 \\
\text { Sept. 10, } 1972 \\
\text { July } 17,1973 \\
\text { Sept. 11, } 1973 \\
\text { Sept. 23, } 1975 \\
\text { July } 23,1976 \\
\text { Nov. } 9,1976\end{array}$ & $\begin{array}{l}26.1 \\
44.8 \\
34.2 \\
34.2 \\
19.5 \\
14.3 \\
17.1\end{array}$ & 7.4 & 2.0 & c \\
\hline & Crawfish River & $\begin{array}{l}\text { NW1/4NW1/4 sec. } 19, \mathrm{~T} .10 \mathrm{~N} ., \text { R. } 13 \text { E., } \\
\text { Dodge County, at sewage-treatment } \\
\text { plant, } 0.8 \text { mi downstream from } \\
\text { U.S. Highway } 16 \text { bridge, } 1.0 \mathrm{mi} \\
\text { southeast of Columbus, Wis. }\end{array}$ & 167 & & & & & \\
\hline UR55 & $\begin{array}{l}\text { Crawfish River } \\
\text { tributary }\end{array}$ & $\begin{array}{l}\text { NW } 1 / 4 \text { SW1 } 1 / 4 \text { sec. } 18, T .10 \text { N., R. } 13 \text { E., } \\
\text { Dodge County, at culvert on State } \\
\text { Highway } 60 \text {, at Columbus, Wis. }\end{array}$ & 1.89 & $\begin{array}{l}\text { Sept. 23, } 1975 \\
\text { July 23, } 1976 \\
\text { Nov. 9, } 1976\end{array}$ & $\begin{array}{l}.03 \\
.08 \\
.32\end{array}$ & $\underline{\mathbf{5}}$ & 5 & \\
\hline UR56 & Maunesha River & $\begin{array}{l}\text { NW1/4NE } 1 / 4 \text { sec. } 15, \text { T. } 8 \text { N., R. } 12 \text { E., } \\
\text { Dane County, at sewage-treatment } \\
\text { plant, at Marshall, Wis. }\end{array}$ & 70.6 & $\begin{array}{l}\text { June 2, } 1972 \\
\text { Sept. 10, } 1972 \\
\text { July 19, } 1973 \\
\text { Sept. 11, } 1973 \\
\text { Sept. 22, } 1975 \\
\text { July 22, } 1976 \\
\text { Oct. 4, } 1976\end{array}$ & $\begin{array}{l}10.9 \\
13.1 \\
10.5 \\
11.5 \\
7.36 \\
2.50 \\
1.36\end{array}$ & 2.4 & .62 & c \\
\hline UR57 I & Maunesha River & $\begin{array}{l}\text { NW1/4NE } 1 / 4 \text { sec. } 7, \text { T. } 8 \text { N., R. } 13 \text { E., } \\
\text { Jefferson County, at bridge on } \\
\text { State Highway } 19 \text {, at Waterloo, Wis. }\end{array}$ & 90.8 & $\begin{array}{l}\text { Sept. } 25,1975 \\
\text { July } 21,1976 \\
\text { Oct. } 4,1976 \\
\text { July } 12,1977\end{array}$ & $\begin{array}{l}11.4 \\
3.75 \\
4.33 \\
2.94\end{array}$ & 4.2 & 1.3 & c \\
\hline UR59 I & Maunesha River & $\begin{array}{l}\text { NW } 1 / 4 \text { SW } 1 / 4 \text { sec. } 5, \text { T. } 8 \text { N., R. } 13 \text { E., } \\
\text { Jefferson County, at sewage- } \\
\text { treatment plant outfall in } \\
\text { Fireman Park, at Waterloo, Wis. }\end{array}$ & 91.3 & $\begin{array}{l}\text { June } 2,1972 \\
\text { Sept. 10, } 1972 \\
\text { July } 18,1973 \\
\text { Sept. 13, } 1973 \\
\text { Sept. 22, } 1975 \\
\text { July } 23,1976 \\
\text { Oct. } 4,1976\end{array}$ & $\begin{array}{l}14.7 \\
18.7 \\
16.3 \\
15.8 \\
9.98 \\
5.20 \\
4.54\end{array}$ & 4.3 & 1.3 & c \\
\hline $\begin{array}{l}\text { UR62, } \\
63\end{array}$ & $\begin{array}{l}\text { Beaverdam } \\
\text { River }\end{array}$ & $\begin{array}{l}\text { NEY/4SW1/4 sec. 4, T. } 11 \text { N., R. } 14 \text { E., } \\
\text { Dodge County, at bridge on Davis } \\
\text { Street, in Beaver Dam, Wis. }\end{array}$ & 157 & $\begin{array}{l}\text { Aug. } 14,1967 \\
\text { June } 2,1972 \\
\text { July } 17,1973 \\
\text { Sept. 11, } 1973 \\
\text { Sept. 23, } 1975 \\
\text { July } 21,1976 \\
\text { Nov. } 10,1976\end{array}$ & $\begin{array}{c}26.0 \\
20.1 \\
3.42 \\
13.0 \\
13.9 \\
.81 \\
.24\end{array}$ & 6 & $\underline{6}$ & \\
\hline UR64 & $\begin{array}{l}\text { Tributary to } \\
\text { Beaverdam } \\
\text { tributary }\end{array}$ & $\begin{array}{l}\text { NE1/1/NE1/4 sec. 35, T. } 13 \text { N., R. } 13 \text { E., } \\
\text { Dodge County, at culvert on } \\
\text { Trenton Street, at Fox Lake, Wis. }\end{array}$ & .27 & $\begin{array}{l}\text { Sept. } 23,1975 \\
\text { July } 21,1976 \\
\text { Nov. 10, } 1976 \\
\text { July } 11,1977\end{array}$ & $\begin{array}{l}.33 \\
.25 \\
.29 \\
.16\end{array}$ & .17 & .06 & c \\
\hline UR65 & $\begin{array}{l}\text { Beaverdam River } \\
\text { tributary }\end{array}$ & $\begin{array}{l}\text { SE1/1/4NE1/4 sec. 35, T. } 13 \text { N., R. } 13 \text { E., } \\
\text { Dodge County, at sewage-treatment } \\
\text { plant, } 0.1 \text { mi downstream from } \\
\text { Chicago, Milwaukee, St. Paul, and } \\
\text { Pacific Railroad, in Fox Lake, Wis. }\end{array}$ & 1.28 & $\begin{array}{l}\text { June } 1,1972 \\
\text { July } 17,1973 \\
\text { Sept. 11, } 1973 \\
\text { Sept. 23, } 1975 \\
\text { July } 21,1976 \\
\text { Nov. 10, } 1976\end{array}$ & $\begin{array}{l}.32 \\
.46 \\
.37 \\
.26 \\
.26 \\
.13\end{array}$ & .12 & .05 & c \\
\hline
\end{tabular}


Table 20. Upper Rock River basin station locations and low-flow characteristics-Continued.

\begin{tabular}{|c|c|c|c|c|c|c|c|c|}
\hline $\begin{array}{l}\text { Station } \\
\text { number }\end{array}$ & ${ }_{\text {Stream }}^{1}$ & Station location & $\begin{array}{l}\text { Drainage } \\
\text { area } \\
\left(\mathrm{mi}^{2}\right)\end{array}$ & Date & $\begin{array}{l}\text { Discharge } \\
\left(\mathrm{ft}^{3} / \mathrm{s}\right)\end{array}$ & $\begin{array}{l}Q_{7,2} Q_{7} \\
\left(\mathrm{ft}^{3 / \mathrm{s}}\right)\left(\mathrm{ft}^{3}\right.\end{array}$ & $3 / 10$ & $\begin{array}{c}\text { Accuracy } \\
\text { level }\end{array}$ \\
\hline UR66 $\frac{E}{t}$ & $\begin{array}{l}\text { Beaver Creek } \\
\text { tributary }\end{array}$ & $\begin{array}{l}\text { SW } 1 / 4 \mathrm{SW} 1 / 4 \text { sec. } 13, \mathrm{~T} .12 \mathrm{~N} ., \mathrm{R} .12 \mathrm{E.} \\
\text { Columbia County, at bridge on } \\
\text { County Trunk G, } 2.4 \text { mi southwest of } \\
\text { Randolph, Wis. }\end{array}$ & 4.58 & $\begin{array}{l}\text { June } 1,1972 \\
\text { July } 17,1973 \\
\text { Sept. 11, 1973 } \\
\text { Sept. 23, } 1975 \\
\text { July } 21,1976 \\
\text { Nov. } 10,1976\end{array}$ & $\begin{array}{l}1.00 \\
.74 \\
.43 \\
.27 \\
.26 \\
.17\end{array}$ & 0.12 & 0.03 & c \\
\hline UR69 & $\begin{array}{l}\text { Beaverdam River } \\
\text { tributary }\end{array}$ & $\begin{array}{l}\text { NW1/4SE1/4 sec. } 28, \text { T. } 10 \text { N., R. } 14 \text { E., } \\
\text { Dodge County, at bridge on County } \\
\text { Trunk J, } 0.2 \text { mi east of Reeseville, } \\
\text { Wis. }\end{array}$ & 3.51 & $\begin{array}{l}\text { June } 2,1972 \\
\text { July } 18,1973 \\
\text { Sept. 13, } 1973 \\
\text { Sept. 23, } 1975 \\
\text { July } 21,1976 \\
\text { Nov. } 9,1976\end{array}$ & $\begin{array}{l}.18 \\
.10 \\
.01 \\
.12 \\
\Sigma_{0}^{.01}\end{array}$ & 0 & 0 & c \\
\hline UR71 $\mathrm{F}$ & Rock Creek & $\begin{array}{l}\text { NW1/4NE } 1 / 4 \text { sec. } 13, T .7 \text { N., R. } 13 \text { E., } \\
\text { Jefferson County, at County Trunk } \\
\text { B, in Lake Mills, Wis. }\end{array}$ & 15.7 & $\begin{array}{l}\text { June } 1,1972 \\
\text { Sept. 10, } 1972 \\
\text { July 19, } 1973 \\
\text { Sept. 13,1973 } \\
\text { Sept. 22, } 1975 \\
\text { July 21, } 1976 \\
\text { Oct. 4, } 1976\end{array}$ & $\begin{array}{l}1.12 \\
1.22 \\
7.24 \\
1.08 \\
2.62 \\
1.09 \\
1.10\end{array}$ & $\underline{I}$ & I & \\
\hline & Rock Creek & $\begin{array}{l}\mathrm{SE} 1 / 4 \mathrm{SE}^{1} / 4 \text { sec. } 12, \mathrm{~T} .7 \mathrm{~N} ., \mathrm{R} .13 \mathrm{E} . \text {, } \\
\text { Jefferson County, at sewage- } \\
\text { treatment plant, } 0.8 \text { mi east of } \\
\text { Lake Mills, Wis. }\end{array}$ & 16.0 & & & & & \\
\hline UR2A & Spring Brook & $\begin{array}{l}\text { SE } 1 / 4 \text { NE } 1 / 4 \text { sec. } 15, \text { T. } 12 \text { N., R. } 15 \text { E., } \\
\text { Dodge County, at culvert on } \\
\text { Burnett Ditch Road, } 1.1 \text { mi north- } \\
\text { east of Burnett, Wis. }\end{array}$ & 8.72 & $\begin{array}{l}\text { Sept. } 24,1975 \\
\text { Nov. 11, } 1976 \\
\text { July } 11,1977\end{array}$ & $\begin{array}{l}2.67 \\
1.22 \\
.86\end{array}$ & 1.0 & .25 & c \\
\hline UR3A & Rock Creek & $\begin{array}{l}\text { NW1/4NW1/4 sec. 13, T. } 7 \text { N., R. } 13 \text { E., } \\
\text { Jefferson County, at State } \\
\text { Highway } 89 \text {, in Lake Mills, Wis. }\end{array}$ & 15.0 & $\begin{array}{l}\text { Oct. } 17,1973 \\
\text { Sept. 22, } 1975 \\
\text { July } 21,1976 \\
\text { Oct. 4, } 1976 \\
\text { July } 12,1977\end{array}$ & $\begin{array}{l}.62 \\
.34 \\
.23 \\
.18 \\
.22\end{array}$ & .22 & .13 & c \\
\hline UR4A & Baker Creek & $\begin{array}{l}\text { NE } 1 / 4 \text { SW } 1 / 4 \text { sec. } 9, \text { T. } 9 \text { N., R. } 16 \text { E., } \\
\text { Dodge County, just upstream of } \\
\text { tributary upstream of County } \\
\text { Trunk MM, } 1.0 \text { mi east of } \\
\text { Lebanon, Wis. }\end{array}$ & 8.63 & $\begin{array}{l}\text { Sept. 26, } 1975 \\
\text { July 21, } 1.976 \\
\text { Nov. 12, } 1976 \\
\text { July } 12,1977\end{array}$ & $\begin{array}{l}4.51 \\
2_{0}^{0} \\
2_{0}^{0}\end{array}$ & 0 & 0 & c \\
\hline UR5A & Gill Creek & $\begin{array}{l}\text { SW } 1 / 4 \text { NW } 1 / 4 \text { sec. } 24, T .13 \text { N., R. } 16 \text { E., } \\
\text { Dodge County, } 0.15 \text { mi downstream } \\
\text { from Dairy Road, } 1.1 \text { mi northeast } \\
\text { of Leroy, Wis. }\end{array}$ & 6.16 & $\begin{array}{l}\text { Sept. 26, } 1975 \\
\text { July 22, } 1976 \\
\text { Nov. 11, } 1976 \\
\text { July } 11,1977\end{array}$ & $\begin{array}{l}.70 \\
.80 \\
.49 \\
.37\end{array}$ & .43 & .25 & c \\
\hline
\end{tabular}

1Based on 1969 report by Wisconsin Department of Natural Resources (DNR). Site lettered "A" was not included in 1969 DNR report.

2 Streambed is dry.

3 Additional discharge measurements are available.

${ }^{4}$ Negligible discharge--water ponded, unable to measure velocity.

${ }^{5}$ No estimate possible--discharge is primarily effluent.

${ }^{6}$ No estimate possible due to regulation upstream.

7 Unable to define relationship. 
Table 21. Root River basin station locations and low-flow characteristics.
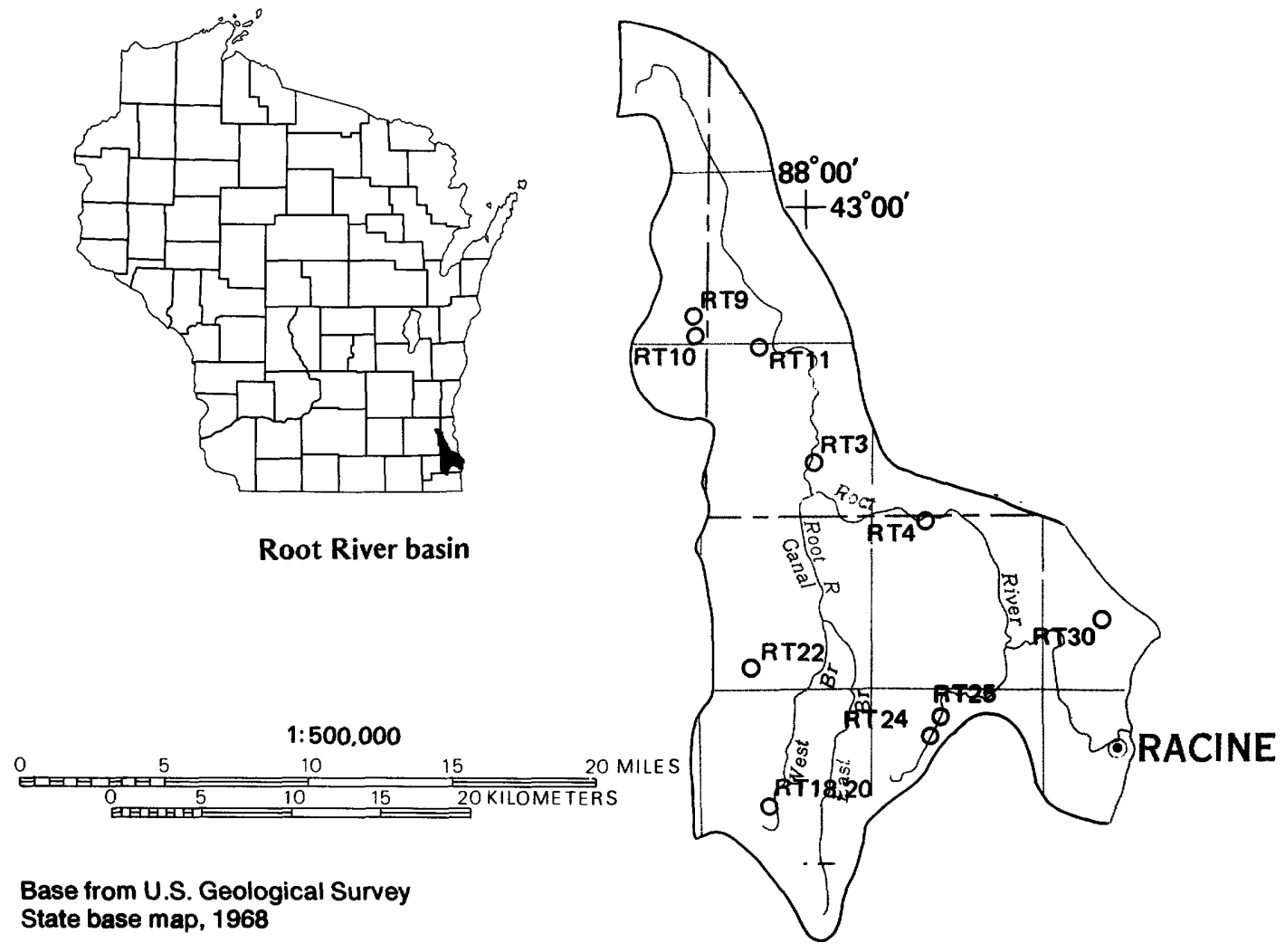

\begin{tabular}{|c|c|c|c|c|c|c|}
\hline $\begin{array}{l}\text { Station }{ }^{1} \\
\text { number }\end{array}$ & $\underset{\text { name }}{\text { Stream }}$ & Station location & $\begin{array}{c}\text { Drainage } \\
\text { area } \\
\left(\mathrm{mi}^{2}\right)\end{array}$ & Date & $\begin{array}{c}\text { Discharge } \\
\left(\mathrm{ft}^{3} / \mathrm{s}\right)\end{array}$ & $\begin{array}{cc}0_{7,2} & 0_{7,10} \\
\left(\mathrm{ft}^{3} / \mathrm{s}\right)\left(\mathrm{ft}^{3} / \mathrm{s}\right) & \text { Accuracy } \\
\text { level } & \end{array}$ \\
\hline
\end{tabular}

RT3 Root River

RT4 Root River

Root River

RT9 Tributary to
Root River tributary

RT10 Tributary to Root River tributary

RT11 Tributary to Root River tributary
SE $1 / 4 S E 1 / 4$ sec. 22, T. 5 N., R. 21 E., Milwaukee County, $400 \mathrm{ft}$ upstream from State Highway 100, $2.4 \mathrm{mi}$ southeast of Franklin, Wis.

NE $1 / 4$ NE $1 / 4$ sec. 5, T. 4 N., R. 22 E., Racine County, at bridge on

State Highway 38, 2.5 mi north of Caledonia, Wis.

NW $1 / 4 N^{1} / \frac{1}{4}$ sec. 4, T. 4 N., R. 22 E., Racine County, at sewage-treatment plant, 2.6 mi northeast of

Caledonia, Wis.

SW1/4SE $1 / 4$ sec. 35, T. 6 N., R. 20 E., Waukesha County, at bridge on County Trunk HH, 2.6 mi west of Hales Corners, Wis.

NW $1 / 4$ SE $1 / 4$ sec. 2, T. 5 N., R. 20 E., Waukesha County, at bridge on State Highway 24, 2.5 mi northeast of Muskego, Wis.

SE $1 / 4$ NW1/4 sec. 7, T. 5 N., R. 21 E., Milwaukee County, at bridge on County Trunk J, 2.0 mi south of Hales Corners, Wis.
47.2

$3.6 \quad 1.6 \quad$ a

150

July 25, 1972

June 12, 1973

Aug. 13, 1973

39.6

64.8

8.56

Sept. 18, 1975

150

\begin{tabular}{|c|c|c|c|c|c|}
\hline 2.68 & $\begin{array}{l}\text { July } 25,1972 \\
\text { June } 13,1973 \\
\text { Aug. } 13,1973 \\
\text { Sept. } 18,1975 \\
\text { Oct. } 4,1976\end{array}$ & $\begin{array}{l}.05 \\
2_{0}^{.25} \\
20 \\
20\end{array}$ & 0 & 0 & $c$ \\
\hline 3.07 & $\begin{array}{l}\text { July } 25,1972 \\
\text { June } 13,1973 \\
\text { Aug. } 13,1973 \\
\text { Sept. } 18,1975 \\
\text { Oct. } 4,1976\end{array}$ & $\begin{array}{l}.10 \\
.55 \\
.04 \\
.02 \\
.02\end{array}$ & .02 & $<.01$ & c \\
\hline .67 & $\begin{array}{l}\text { July } 25,1972 \\
\text { June } 13,1973 \\
\text { Aug. } 13,1973 \\
\text { Sept. } 18,1975 \\
\text { Oct. } 4,1976\end{array}$ & $\begin{array}{l}.35 \\
1.78 \\
.11 \\
.46 \\
.28\end{array}$ & .03 & $<.01$ & 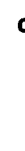 \\
\hline
\end{tabular}


Table 21. Root River basin station locations and low-flow characteristics-Continued.

\begin{tabular}{|c|c|c|c|c|c|c|c|c|}
\hline $\begin{array}{l}\text { Station } \\
\text { number }\end{array}$ & ${ }^{1} \underset{\text { name }}{\text { Stream }}$ & Station location & $\begin{array}{c}\text { Drainage } \\
\text { area } \\
\left(\mathrm{mi}^{2}\right)\end{array}$ & Date & $\begin{array}{c}\text { Discharge } \\
\left(\mathrm{ft}^{3} / \mathrm{s}\right)\end{array}$ & $\begin{array}{l}\mathrm{Q}_{7,2} \mathrm{Q}_{7} \\
\left(\mathrm{ft}^{3} / \mathrm{s}\right)(\mathrm{ft}\end{array}$ & $\begin{array}{l}7,10 \\
\left.t^{3} / \mathrm{s}\right)\end{array}$ & $\begin{array}{c}\text { Accuracy } \\
\text { level }\end{array}$ \\
\hline $\begin{array}{l}\text { RT 18, } \\
20\end{array}$ & $\begin{array}{l}\text { West Branch } \\
\text { Root River } \\
\text { Canal }\end{array}$ & $\begin{array}{l}\text { NEY/4SE1/4 sec. } 29, \text { T. } 3 \text { N., R. } 21 \text { E., } \\
\text { Racine County, at culvert on } 67 \text { th } \\
\text { Drive, } 1.0 \text { mi east of Union Grove, } \\
\text { Wis. }\end{array}$ & 3.94 & $\begin{array}{l}\text { July } 24,1972 \\
\text { June } 12,1973 \\
\text { Aug. } 13,1973 \\
\text { Sept. 18, } 1975 \\
\text { Oct. } 4,1976\end{array}$ & $\begin{array}{l}0.70 \\
3.05 \\
.38 \\
.99 \\
1.11\end{array}$ & $\underline{7}$ & $\underline{7}$ & \\
\hline RT22 & $\begin{array}{l}\text { West Branch } \\
\text { Root River } \\
\text { Canal tributary }\end{array}$ & $\begin{array}{l}\text { SWY } 1 \text { SW1/4 sec. } 34, \text { T. } 4 \text { N., R. } 21 \text { E., } \\
\text { Racine County, at culvert on Twomile } \\
\text { Road, } 3.3 \text { mi southeast of North } \\
\text { Cape, Wis. }\end{array}$ & 4.69 & $\begin{array}{l}\text { Sept. 18, } 1975 \\
\text { Oct. 4, } 1976 \\
\text { Sept. } 6,1978\end{array}$ & $\begin{array}{l}.07 \\
.10 \\
.45\end{array}$ & 0.06 & 0.02 & c \\
\hline RT24 & Hoods Creek & $\begin{array}{l}\text { SW } 1 / 4 \text { NE } 1 / 4 \text { sec. } 17, T .3 \text { N., R. } 22 \text { E., } \\
\text { Racine County, at bridge on State } \\
\text { Highway } 20,2.1 \text { mi northwest of } \\
\text { Sturtevant, Wis. }\end{array}$ & 3.38 & $\begin{array}{l}\text { July } 24,1972 \\
\text { June } 12,1973 \\
\text { Aug. } 13,1973 \\
\text { Sept. 18, } 1975 \\
\text { Oct. } 4,1976\end{array}$ & $\begin{array}{l}1.38 \\
1.35 \\
.01 \\
2.02\end{array}$ & 0 & 0 & c \\
\hline RT25 & Hoods Creek & $\begin{array}{l}\text { NW1/4NW1/4 sec. } 9, \text { T. } 3 \text { N., R. } 22 \text { E., } \\
\text { Racine County, at bridge on County } \\
\text { Trunk C, } 2.9 \text { mi north of Sturtevant, } \\
\text { Wis. }\end{array}$ & 8.84 & $\begin{array}{l}\text { Sept. 18, } 1975 \\
\text { Oct. 4, } 1976 \\
\text { Sept. 6, } 1978\end{array}$ & $\begin{array}{r}.05 \\
<.01 \\
3.04\end{array}$ & $<.01$ & $<.01$ & c \\
\hline RT30 & $\begin{array}{l}\text { Lake Michigan } \\
\text { tributary }\end{array}$ & $\begin{array}{l}\text { SE } 1 / 4 \text { SE } 1 / 4 \text { sec. } 21, \text { T. } 4 \text { N., R. } 23 \text { E., } \\
\text { Racine County, at culvert on road } \\
\text { to sewage-treatment plant, } 0.8 \mathrm{mi} \\
\text { west of Wind Point, Wis. }\end{array}$ & 1.03 & $\begin{array}{l}\text { July } 24,1972 \\
\text { June } 12,1973 \\
\text { Aug. } 13,1973 \\
\text { Sept. 18, } 1975 \\
\text { Oct. } 4,1976\end{array}$ & $\begin{array}{l}2.14 \\
2.75 \\
.43 \\
.05 \\
1.62\end{array}$ & .08 & .02 & c \\
\hline
\end{tabular}

${ }^{1}$ Based on 1967 report by Wisconsin Department of Natural Resources.

${ }^{2}$ Streambed is dry.

7 Unable to define relationship. 
Table 22. St. Croix River basin station locations and low-flow characteristics.

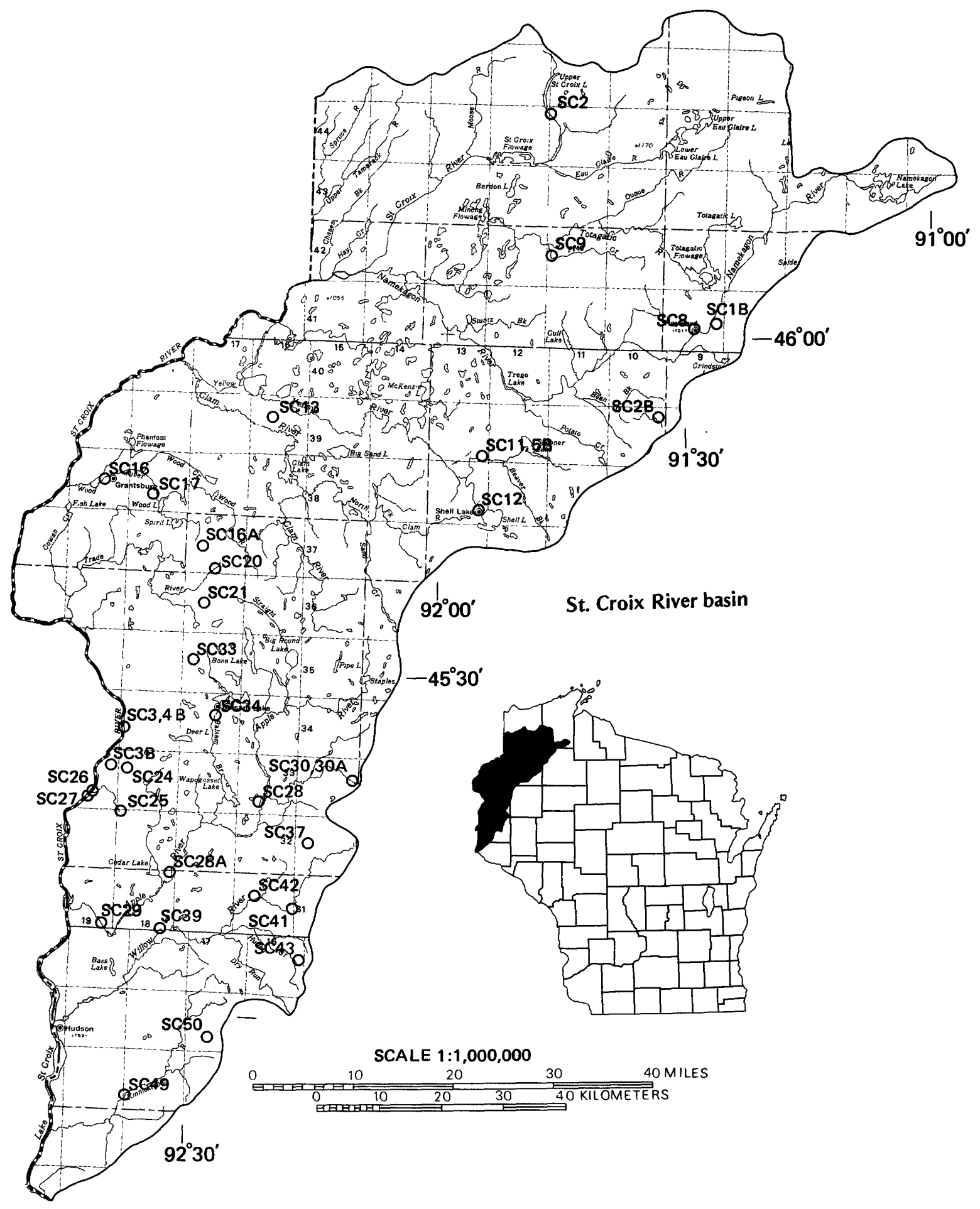

Base from U.S. Geological Survey State base map, 1968 
Table 22. St. Croix River basin station locations and low-flow characteristics-Continued.

\begin{tabular}{|c|c|c|c|c|c|c|c|c|}
\hline $\begin{array}{l}\text { Station } \\
\text { number }\end{array}$ & Stream & Station location & $\begin{array}{l}\text { Drainage } \\
\text { area } \\
\left(\mathrm{mi}^{2}\right)\end{array}$ & Date & $\begin{array}{c}\text { Discharge } \\
\left(\mathrm{ft}^{3} / \mathrm{s}\right)\end{array}$ & $\begin{array}{l}Q_{7,2} \\
\left(f^{3} / s\right)(\end{array}$ & $\begin{array}{l}Q_{7,10} \\
\left(\mathrm{ft}^{3} / \mathrm{s}\right)\end{array}$ & $\underset{\text { Aevel }}{\text { Accuracy }}$ \\
\hline $\mathrm{SC} 2$ & St. Croix River & $\begin{array}{l}\text { NW1/4SE } 1 / 4 \text { sec. } 7, \text { T. } 44 \text { N., R. } 11 \text { W., } \\
\text { Douglas County, at bridge on } \\
\text { country road, } 3.6 \text { mi southeast } \\
\text { of Solon Springs, Wis. }\end{array}$ & 36.9 & $\begin{array}{l}\text { July } 23,1974 \\
\text { June } 8,1976 \\
\text { Aug. } 4,1976 \\
\text { Sept. 29, } 1976 \\
\text { Aug. 24, } 1977\end{array}$ & $\begin{array}{l}11.5 \\
16.7 \\
10.3 \\
17.0 \\
21.7\end{array}$ & 15 & 11 & c \\
\hline $\begin{array}{l}\mathrm{SC} 3 . \\
4 \mathrm{~B}\end{array}$ & St. Croix River & $\begin{array}{l}\text { SW1/4NW1/4 sec. } 30, \text { T. } 34 \text { N., R. } 18 \text { W., } \\
\text { Polk County, on left bank } 0.3 \mathrm{mi} \\
\text { downstream from powerplant, at } \\
\text { St. Croix Falls, Wis. }\end{array}$ & 5,930 & & & 1,490 & 1,080 & $\mathbf{a}$ \\
\hline SC8 & Namekagon River & $\begin{array}{l}\text { NE } 1 / 4 S^{-1} / 4 \text { sec. } 28, \text { T. } 41 \text { N., R. } 9 \text { W., } \\
\text { Sawyer County, at ranger station, } \\
0.8 \text { mi southwest of U.S. Highway } \\
63 \text { and State Highway } 27 \text { in } \\
\text { Hayward, Wis. }\end{array}$ & 231 & $\begin{array}{l}\text { May } 22,1973 \\
\text { Sept. 19, } 1973 \\
\text { Oct. } 17,1973 \\
\text { July } 23,1974 \\
\text { Aug. } 12,1975 \\
\text { June } 8,1976 \\
\text { Sept. } 30,1976\end{array}$ & $\begin{array}{l}282 \\
203 \\
304 \\
107 \\
156 \\
179 \\
128\end{array}$ & 130 & 100 & c \\
\hline sc9 & Frog Creek & $\begin{array}{l}\text { NE } 1 / 4 N W 1 / 4 \text { sec. } 19, T .42 \text { N., R. } 11 \text { W., } \\
\text { Washburn County, at bridge on } \\
\text { country road, } 1.8 \text { mi northeast } \\
\text { of Minong. Wis. }\end{array}$ & 46.1 & $\begin{array}{l}\text { Aug. } 22,1967 \\
\text { Aug. } 21,1969 \\
\text { Aug. } 25,1970 \\
\text { May } 21,1971 \\
\text { Oct. } 13,1971 \\
\text { May } 22,1973 \\
\text { Sept. } 20,1973 \\
\text { June } 26,1974 \\
\text { July } 23,1974 \\
\text { Aug. } 12,1975 \\
\text { June } 8,1976\end{array}$ & $\begin{array}{l}4.99 \\
3.83 \\
2.05 \\
26.1 \\
16.6 \\
26.2 \\
6.60 \\
12.3 \\
16.9 \\
2.96 \\
3.88\end{array}$ & 3.1 & 1.4 & c \\
\hline \multirow[t]{2}{*}{$\begin{array}{l}\text { SC11, } \\
5 B\end{array}$} & Yellow River & $\begin{array}{l}\mathrm{SW} 1 / \mathrm{SE} 1 / 4 \text { sec. } 36, \mathrm{~T} .39 \mathrm{~N} ., \mathrm{R} .13 \mathrm{~W} . \text {. } \\
\text { Washburn County, at sewage- } \\
\text { treatment pond, } 1.3 \text { mi west of } \\
\text { Spooner, Wis. }\end{array}$ & 77.9 & & & & & \\
\hline & Yellow River & $\begin{array}{l}\text { NE } 1 / 4 S^{1} 1 / 4 \text { sec. } 35, \text { T. } 39 \text { N., R. } 13 \text { W., } \\
\text { Washburn County, at Tozer Lake } \\
\text { Road, } 2.0 \text { mi west of Spooner, Wis. }\end{array}$ & 81.9 & $\begin{array}{l}\text { Aug. } 16,1972 \\
\text { May } 21,1973 \\
\text { Sept. } 25,1973 \\
\text { July } 24,1974 \\
\text { Aug. } 11,1975 \\
\text { June } 9,1976\end{array}$ & $\begin{array}{l}46.0 \\
54.6 \\
70.0 \\
48.0 \\
33.2 \\
30.9\end{array}$ & 33 & 27 & c \\
\hline \multirow[t]{2}{*}{ sc12 } & Sawyer Creek & $\begin{array}{l}\text { SWY/4NW1/4 sec. } 25, \text { T. } 38 \text { N., R. } 13 \text { W., } \\
\text { Washburn County, at sewage-disposal } \\
\text { plant, at Shell Lake, Wis. }\end{array}$ & 26.2 & & & & & \\
\hline & Sawyer Creek & $\begin{array}{l}\text { NW1/4NEY// sec. } 14, \text { T. } 38 \text { N., R. } 13 \text { W., } \\
\text { Washburn County, at culvert on } \\
\text { country road, } 2.8 \text { mi north of } \\
\text { Shell Lake, Wis. }\end{array}$ & 33.7 & $\begin{array}{lc}\text { Aug. } & 16,1972 \\
\text { May } & 21,1973 \\
\text { Sept. } 25,1973 \\
\text { July } 24,1974 \\
\text { Aug. } 11,1975 \\
\text { June } 9,1976\end{array}$ & $\begin{array}{l}8.69 \\
7.56 \\
6.68 \\
6.51 \\
5.29 \\
4.10\end{array}$ & 4.3 & 3.2 & c \\
\hline \multirow[t]{2}{*}{$\mathrm{sC13}$} & $\begin{array}{l}\text { Clam River } \\
\text { tributary }\end{array}$ & $\begin{array}{l}\text { SE1/4NW1/4 sec. } 16, T .39 \text { N., R. } 16 \text { W., } \\
\text { Burnett County, at sewage-stabilization } \\
\text { pond, } 0.5 \text { mi southeast of Webster, } \\
\text { Wis. }\end{array}$ & .47 & & & & & \\
\hline & $\begin{array}{l}\text { Clam River } \\
\text { tributary }\end{array}$ & $\begin{array}{l}\text { SW } 1 / 4 \text { SE } 1 / 4 \text { sec. } 16, \text { T. } 39 \text { N., R. } 16 \text { W., } \\
\text { Burnett County, at culvert on } \\
\text { Bass Lake Road, } 1.2 \text { mi southeast } \\
\text { of Webster, Wis. }\end{array}$ & 1.54 & $\begin{array}{l}\text { Aug. } 16,1972 \\
\text { May } 24,1973 \\
\text { Sept. } 25,1973 \\
\text { July } 25,1974 \\
\text { July } 30,1975 \\
\text { June } 9,1976\end{array}$ & $\begin{array}{l}.30 \\
1.87 \\
4_{0}^{.65} \\
4_{0}^{0} \\
<.01\end{array}$ & 0 & 0 & c \\
\hline Sc16 & Wood River & $\begin{array}{l}\text { SE } 1 / 4 \text { SWY/4 sec. } 15, T .38 \text { N., R. } 19 \text { W., } \\
\text { Burnett County, at bridge on State } \\
\text { Highway } 70,1.3 \text { mi west of } \\
\text { Grantsburg, Wis. }\end{array}$ & 163 & $\begin{array}{lr}\text { May } & 23,1973 \\
\text { Sept. } 25,1973 \\
\text { Oct. } 17,1973 \\
\text { July } 25,1974 \\
\text { July } 30,1975 \\
\text { June } 9,1976\end{array}$ & $\begin{array}{c}96.8 \\
59.1 \\
130 \\
42.1 \\
40.6 \\
37.5\end{array}$ & 34 & 25 & c \\
\hline
\end{tabular}


Table 22. St. Croix River basin station locations and low-flow characteristics-Continued.

\begin{tabular}{|c|c|c|c|c|c|c|c|c|}
\hline $\begin{array}{l}\text { Station } \\
\text { number }\end{array}$ & $1 \underset{\text { name }}{\text { Stream }}$ & Station location & $\begin{array}{l}\text { Drainage } \\
\text { area } \\
\left(\mathrm{mi}^{2}\right)\end{array}$ & Date & $\begin{array}{c}\text { Discharge } \\
\left(\mathrm{ft}^{3} / \mathrm{s}\right)\end{array}$ & $\begin{array}{l}\mathrm{a}_{7,2} \mathrm{a}_{7} \\
\left(\mathrm{ft}^{3} / \mathrm{s}\right)\left(\mathrm{ft}^{3}\right.\end{array}$ & \multicolumn{2}{|c|}{ (s) Accuracy } \\
\hline SC16A & $\begin{array}{l}\text { Wood River } \\
\text { tributary }\end{array}$ & $\begin{array}{l}\text { SW1/4SE1/4 sec. } 20, T .37 \mathrm{~N} ., \mathrm{R} .17 \mathrm{~W} . \\
\text { Polk County, at culvert on County } \\
\text { Trunk W, } 1.9 \text { mi northwest of } \\
\text { Frederic, Wis. }\end{array}$ & 1.78 & $\begin{array}{l}\text { July } 23,1974 \\
\text { Aug. } 5,1976 \\
\text { Sept. 28, } 1976\end{array}$ & $\begin{array}{l}0.72 \\
.33 \\
.37\end{array}$ & 0.46 & 0.25 & c \\
\hline $\operatorname{sc17} V$ & Wood River & $\begin{array}{l}\text { NE1/4NE } 1 / 4 \text { sec. } 28, T .38 \mathrm{~N} ., \mathrm{R} .18 \mathrm{~W} . \text {., } \\
\text { Burnett County, at culvert on } \\
\text { country road, } 1.2 \text { mi southwest of } \\
\text { Alpha, Wis. }\end{array}$ & 72.7 & $\begin{array}{l}\text { July } 23,1974 \\
\text { Aug. } 5,1976 \\
\text { Sept. } 28,1976\end{array}$ & $\begin{array}{l}23.1 \\
16.2 \\
14.9\end{array}$ & 18 & 12 & c \\
\hline $\operatorname{sc20}$ & $\begin{array}{l}\text { Trade River } \\
\text { tributary }\end{array}$ & $\begin{array}{l}\text { SW1/4NW1/4 sec. 34, T. } 37 \mathrm{~N} ., \mathrm{R} .17 \mathrm{~W} . \text {., } \\
\text { Polk County, at culvert on State } \\
\text { Highways } 35 \text { and } 48,0.6 \mathrm{mi} \text { south } \\
\text { of Frederic, Wis. }\end{array}$ & 1.56 & $\begin{array}{l}\text { Aug. } 15,1972 \\
\text { May } 23,1973 \\
\text { Sept. 25, } 1973 \\
\text { July 23, } 1974 \\
\text { July } 30,1975 \\
\text { June } 10,1976\end{array}$ & $\begin{array}{r}.17 \\
.08 \\
.02 \\
.01 \\
<.01 \\
.04\end{array}$ & $<.01$ & $<.01$ & c \\
\hline \multirow[t]{2}{*}{ Sc21 } & $\begin{array}{l}\text { Trade River } \\
\text { tributary }\end{array}$ & $\begin{array}{l}\text { NW1/4SW1/4 sec. } 28, \text { T. } 36 \text { N., R. } 17 \text { W., } \\
\text { Polk County, at sewage-treatment } \\
\text { plant, at Luck, Wis. }\end{array}$ & .08 & & & & & \\
\hline & $\begin{array}{l}\text { Trade River } \\
\text { tributary }\end{array}$ & $\begin{array}{l}\text { NW } 1 / 4 \text { NW } 1 / 4 \text { sec. } 21, T .36 \text { N., R. } 17 \text { W., } \\
\text { Polk County, at culvert on country } \\
\text { road, } 1.7 \text { mi north of Luck, Wis. }\end{array}$ & 2.13 & $\begin{array}{l}\text { Aug. } 15,1972 \\
\text { May } 24,1973 \\
\text { Sept. } 25,1973 \\
\text { July } 23,1974 \\
\text { July } 30,1975 \\
\text { June } 9,1976\end{array}$ & $\begin{array}{l}1.04 \\
8.31 \\
.85 \\
.39 \\
.52 \\
.56\end{array}$ & .15 & .05 & c \\
\hline SC24 & $\begin{array}{l}\text { St. Croix } \\
\text { River tributary }\end{array}$ & $\begin{array}{l}\text { SE } 1 / 4 \text { SW } 1 / 4 \text { sec. } 7, T .33 \mathrm{~N} ., \mathrm{R} .18 \mathrm{~W} . \text {., } \\
\text { Polk County, just upstream from } \\
\text { sewage-treatment plant, } 0.4 \mathrm{mi} \\
\text { west of Dresser, Wis. }\end{array}$ & 4.95 & $\begin{array}{l}\text { Aug. } 14,1972 \\
\text { May } 24,1973 \\
\text { Sept. } 25,1973 \\
\text { July } 24,1974 \\
\text { July } 29,1975 \\
\text { June } 9,1976\end{array}$ & $\begin{array}{l}.74 \\
.30 \\
.20 \\
.23 \\
.06\end{array}$ & 0 & 0 & c \\
\hline SC25 7 & Trout Brook & $\begin{array}{l}\text { SE1/4SE1/4 sec. } 36, \mathrm{~T} .33 \mathrm{~N} ., \mathrm{R} .19 \mathrm{~W} . \text {., } \\
\text { Polk County, at culvert on town } \\
\text { road, } 3.0 \mathrm{mi} \text { southeast of } \\
\text { Osceola, Wis. }\end{array}$ & 6.16 & $\begin{array}{l}\text { July } 24,1974 \\
\text { July } 29,1975 \\
\text { June } 9,1976 \\
\text { Aug. } 3,1976 \\
\text { Sept. 28, } 1976\end{array}$ & $\begin{array}{l}.94 \\
1.51 \\
1.61 \\
.78 \\
1.21\end{array}$ & .59 & .24 & c \\
\hline SC26 & Trout Brook & $\begin{array}{l}\text { NE } 1 / 4 N E 1 / 4 \text { sec. } 27, T .33 \text { N., R. } 19 \text { W., } \\
\text { Polk County, at bridge on County } \\
\text { Trunk M, at Osceola, Wis. }\end{array}$ & 15.7 & $\begin{array}{l}\text { July } 24,1974 \\
\text { June } 9,1976 \\
\text { Aug. } 6,1976 \\
\text { Sept. 28, } 1976\end{array}$ & $\begin{array}{l}4.51 \\
5.26 \\
3.39 \\
3.38\end{array}$ & 2.4 & 1.0 & c \\
\hline$S C 27 \quad S$ & $\begin{array}{l}\text { St. Croix } \\
\text { River tributary }\end{array}$ & $\begin{array}{l}\text { NW } 1 / 4 \text { SW } 1 / 4 \text { sec. } 27, T .33 \text { N., R. } 19 \text { W., } \\
\text { Polk County, just upstream from } \\
\text { sewage-treatment plant, at } \\
\text { Osceola, Wis. }\end{array}$ & .15 & $\begin{array}{l}\text { Aug. } 14,1972 \\
\text { May 24, 1973 } \\
\text { Sept. 25, 1973 } \\
\text { Oct. 18, 1973 } \\
\text { July } 24,1974 \\
\text { July 29, 1975 } \\
\text { June } 9,1976\end{array}$ & $\begin{array}{l}20 \\
1.15 \\
.21 \\
.10 \\
.13 \\
.06 \\
.20 \text { est }\end{array}$ & t. & $<.01$ & c \\
\hline $\mathrm{SC} 28$ & Apple River & $\begin{array}{l}\text { NW1/SW1/4 sec. } 33, T .33 \text { N., R. } 16 \text { W., } \\
\text { Polk County, just upstream from } \\
\text { sewage-treatment plant, on town } \\
\text { road, } 0.1 \text { mi southeast of junction } \\
\text { of County Trunk F and State } \\
\text { Highway } 46 \text {, at Amery, Wis. }\end{array}$ & 256 & $\begin{array}{l}\text { Aug. } 14,1972 \\
\text { May 29, } 1973 \\
\text { July 24, } 1974 \\
\text { July 29, } 1975 \\
\text { June 10, } 1976 \\
\text { Aug. } 3,1976 \\
\text { Sept. 28, 1976 }\end{array}$ & $\begin{array}{c}238 \\
459 \\
94.8 \\
112 \\
90.2 \\
60.5 \\
75.1\end{array}$ & 49 & 23 & c \\
\hline SC28A $A$ & Apple River & $\begin{array}{l}\text { SE } 1 / 4 \text { SE } 1 / 4 \text { sec. } 1, T .31 \text { N., R. } 18 \text { W., } \\
\text { St. Croix County, at bridge on } \\
\text { County Trunk HH, at Star Prairie, } \\
\text { Wis. }\end{array}$ & 422 & $\begin{array}{l}\text { July } 24,1974 \\
\text { July } 29,1975 \\
\text { June } 9,1976 \\
\text { Aug. } 3,1976 \\
\text { Sept. 28, } 1976\end{array}$ & $\begin{array}{l}169 \\
210 \\
194 \\
119 \\
151\end{array}$ & 100 & 53 & c \\
\hline
\end{tabular}


Table 22. St. Croix River basin station locations and low-flow characteristics-Continued.

\begin{tabular}{|c|c|c|c|c|c|c|c|c|}
\hline $\begin{array}{l}\text { Station } \\
\text { number }\end{array}$ & ${ }_{\text {name }}^{1}$ & Station location & $\begin{array}{l}\text { Drainage } \\
\text { area } \\
\left(\mathrm{mi}^{2}\right)\end{array}$ & Date & $\begin{array}{l}\text { Discharge } \\
\left(\mathrm{ft}^{3} / \mathrm{s}\right)\end{array}$ & $\begin{array}{l}a_{7,2} Q_{7} \\
\left(\mathrm{ft}^{3} / \mathrm{s}\right)\left(\mathrm{ft}^{3}\right.\end{array}$ & $3 / s)$ & $\begin{array}{c}\text { Accuracy } \\
\text { level }\end{array}$ \\
\hline \multirow[t]{2}{*}{ SC29 } & Apple River & $\begin{array}{l}\text { SE } 1 / 4 \text { SEY } / 4 \text { sec. } 35, \text { T. } 31 \text { N., R. } 19 \text { W., } \\
\text { St. Croix County, at bridge on } \\
\text { State Highways } 35 \text { and } 64 \text {, in } \\
\text { Somerset, Wis. }\end{array}$ & 550 & & & & & \\
\hline & Apple River & $\begin{array}{l}\text { NE } 1 / 4 S^{1} / 4 \text { sec. } 21, \text { T. } 31 \text { N., R. } 19 \text { W., } \\
\text { St. Croix County, at powerplant of } \\
\text { NSP Co., } 3.1 \text { mi northwest of } \\
\text { Somerset, Wis. }\end{array}$ & 555 & & & 142 & 77 & a \\
\hline $\begin{array}{l}\text { Sc30, } \\
30 A\end{array}$ & $\begin{array}{l}\text { South Branch } \\
\text { Beaver Brook } \\
\text { tributary }\end{array}$ & $\begin{array}{l}\text { NW1/SW } 1 / 4 \text { sec. } 11, T .33 \text { N., R. } 15 \text { W., } \\
\text { Polk County, at culvert on country } \\
\text { road, } 2.2 \text { mi northwest of Clayton, } \\
\text { Wis. }\end{array}$ & 2.73 & $\begin{array}{l}\text { Aug. } 14,1972 \\
\text { May } 29,1973 \\
\text { July } 24,1974 \\
\text { July } 29,1975 \\
\text { June } 10,1976 \\
\text { Aug. } 3,1976\end{array}$ & $\begin{array}{l}2.06 \\
2.08 \\
4.48 \\
0 \\
.85 \\
.21\end{array}$ & .07 & .01 & c \\
\hline \multirow[t]{2}{*}{$\operatorname{sc} 33$} & Balsam Branch & $\begin{array}{l}\text { NW } 1 / 4 \text { SW } 1 / 4 \text { sec. } 17, \text { T. } 35 \mathrm{~N} ., \mathrm{R} .17 \mathrm{~W} \text {., } \\
\text { Polk County, at sewage-treatment } \\
\text { plant, } 0.5 \text { mi south of Milltown, } \\
\text { Wis. }\end{array}$ & 2.68 & & & & & \\
\hline & Balsam Branch & $\begin{array}{l}\text { SE1/4SW1/4 sec. } 17, \mathrm{~T} .35 \mathrm{~N} ., \mathrm{R} .17 \mathrm{~W} . \text {, } \\
\text { Polk County, at bridge on town } \\
\text { road, } 1.0 \mathrm{mi} \text { south of Milltown. } \\
\text { Wis. }\end{array}$ & 4.40 & $\begin{array}{l}\text { Aug. } 15,1972 \\
\text { May } 24,1973 \\
\text { July } 23,1974 \\
\text { July } 30,1975 \\
\text { June } 9,1976 \\
\text { Sept. } 28,1976\end{array}$ & $\begin{array}{l}.60 \\
.76 \\
.31 \\
.24 \\
.66 \\
.30\end{array}$ & .17 & .07 & c \\
\hline SC34 & Balsam Branch & $\begin{array}{l}\text { NE1/4SE1/4 sec. 10, T. } 34 \text { N., R. } 17 \text { W., } \\
\text { Polk County, just upstream from } \\
\text { sewage-treatment plant, at south } \\
\text { side of Balsam Lake, Wis. }\end{array}$ & 44.6 & $\begin{array}{l}\text { Aug. } 15,1972 \\
\text { May } 24,1973 \\
\text { July } 23,1974 \\
\text { July } 30,1975 \\
\text { June } 10,1976 \\
\text { Aug. } 3,1976\end{array}$ & $\begin{array}{c}23.0 \\
28.0 \\
5.66 \\
14.6 \\
1.78 \\
1.94\end{array}$ & 1.5 & .36 & c \\
\hline sc37 & $\begin{array}{l}\text { Willow River } \\
\text { tributary }\end{array}$ & $\begin{array}{l}\text { SE1/4NW1/4 sec. } 20, T .32 \text { N., R. } 15 \text { W., } \\
\text { Polk County, at bridge on town } \\
\text { road, at Clear Lake, Wis. }\end{array}$ & 1.93 & $\begin{array}{l}\text { Aug. } 14,1972 \\
\text { May } 29,1973 \\
\text { Sept. } 26,1973 \\
\text { July } 24,1974 \\
\text { July } 29,1975 \\
\text { June } 9,1976\end{array}$ & $\begin{array}{l}{ }_{0} 0 \\
2_{0}^{.24} \\
2_{0}^{2} \\
2_{0}^{0}\end{array}$ & 0 & 0 & c \\
\hline sc39 & Willow River & $\begin{array}{l}\text { SW1/4SE1/4 sec. 35, T. } 31 \text { N., R. } 18 \text { W., } \\
\text { St. Croix County, just downstream } \\
\text { from sewage-treatment plant, } 0.9 \text { mi } \\
\text { west of New Richmond, Wis. }\end{array}$ & 205 & $\begin{array}{l}\text { Aug. } 17,1972 \\
\text { May } 24,1973 \\
\text { Sept. } 24,1973 \\
\text { July } 25,1974 \\
\text { July } 29,1975 \\
\text { June } 9,1976\end{array}$ & $\begin{array}{c}42.5 \\
102 \\
48.8 \\
35.6 \\
47.4 \\
49.6\end{array}$ & 20 & 11 & c \\
\hline SC41 & $\begin{array}{l}\text { Willow River } \\
\text { tributary }\end{array}$ & $\begin{array}{l}\text { SW1/4NW1/4 sec. } 30, \text { T. } 31 \text { N., R. } 15 \text { W., } \\
\text { St. Croix County, at bridge on } \\
\text { U.S. Highway } 63,1.2 \text { mi northwest } \\
\text { of Forest, Wis. }\end{array}$ & 7.42 & $\begin{array}{l}\text { July } 24,1974 \\
\text { July } 28,1975 \\
\text { June } 8,1976 \\
\text { Aug. } 3,1976 \\
\text { Sept. } 27,1976\end{array}$ & $\begin{array}{l}{ }_{4}^{0} \\
4_{0}^{0} \\
4_{0}^{0} \\
{ }^{0}{ }_{0}^{0}\end{array}$ & 0 & 0 & c \\
\hline SC42 & Willow River & $\begin{array}{l}\text { SW1/4NW1/4 sec. 16, T. } 31 \text { N., R. } 16 \text { W., } \\
\text { St. Croix County, at bridge on } \\
\text { country road, } 1.5 \text { mi southeast of } \\
\text { Deer Park, Wis. }\end{array}$ & 75.9 & $\begin{array}{l}\text { July } 24,1974 \\
\text { July } 29,1975 \\
\text { June } 10,1976 \\
\text { Aug. } 3,1976 \\
\text { Sept. } 27,1976\end{array}$ & $\begin{array}{l}10.5 \\
15.6 \\
17.1 \\
12.3 \\
17.2\end{array}$ & 6.4 & 2.9 & c \\
\hline $\mathrm{SC43}$ & $\begin{array}{l}\text { Hutton Creek } \\
\text { tributary }\end{array}$ & $\begin{array}{l}\text { NWy/4SE1/4 sec. } 13, \text { T. } 30 \text { N., R. } 16 \text { W., } \\
\text { St. Croix County, at bridge on } \\
\text { town road, at Emerald, Wis. }\end{array}$ & 5.83 & $\begin{array}{l}\text { Aug. } 17,1972 \\
\text { May } 24,1973 \\
\text { Sept. } 26,1973 \\
\text { Oct. } 18,1973 \\
\text { July } 24,1974 \\
\text { July } 29,1975 \\
\text { June } 8,1976\end{array}$ & $\begin{array}{l}{ }^{4} 0 \\
4^{.88} \\
4_{0}^{0} \\
2_{0}^{0} \\
2_{0}^{0} \\
2_{0}^{0}\end{array}$ & 0 & 0 & c \\
\hline
\end{tabular}


Table 22. St. Croix River basin station locations and low-flow characteristics-Continued.

\begin{tabular}{|c|c|c|c|c|c|c|c|c|}
\hline $\begin{array}{l}\text { Station } \\
\text { number }\end{array}$ & Stream & Station location & $\begin{array}{l}\text { Drainage } \\
\text { area } \\
\left(\mathbf{m i}^{2}\right)\end{array}$ & Date & $\begin{array}{c}\text { Discharge } \\
\left(\mathrm{ft}^{3} / \mathrm{s}\right)\end{array}$ & $\begin{array}{l}\mathrm{a}_{7,2} \mathrm{a}_{7,1} \\
\left(\mathrm{ft}^{3} / \mathrm{s}\right)\left(\mathrm{ft}^{3}\right)\end{array}$ & & $\begin{array}{c}\text { Accuracy } \\
\text { level }\end{array}$ \\
\hline \multirow[t]{2}{*}{ SC49 } & $\begin{array}{l}\text { Kinnickinnic } \\
\text { River }\end{array}$ & $\begin{array}{l}\text { NE1//2NE1/4 sec. 36, T. } 28 \text { N., R. } 19 \text { W., } \\
\text { St. Croix County, at State Highway } \\
35,1.2 \text { mi north of River Falls, Wis. }\end{array}$ & 95.8 & $\begin{array}{l}\text { Aug. } 20,1969 \\
\text { Aug. } 17,1972 \\
\text { May } 24,1973 \\
\text { Sept. } 24,1973 \\
\text { July } 25,1974 \\
\text { July } 28,1975 \\
\text { June } 8,1976\end{array}$ & $\begin{array}{l}32.4 \\
43.3 \\
57.2 \\
40.4 \\
38.0 \\
56.0 \\
47.9\end{array}$ & 26 & 18 & c \\
\hline & $\begin{array}{l}\text { Kinnickinnic } \\
\text { River }\end{array}$ & $\begin{array}{l}\text { SE } 1 / 4 \text { SE } 1 / 4 \text { sec. } 2, \text { T. } 27 \text { N., R. } 19 \text { W., } \\
\text { Pierce County, at sewage-treatment } \\
\text { plant, at River Falls, Wis. }\end{array}$ & 117 & & & & & \\
\hline SC50 & $\begin{array}{l}\text { Kinnickinnic } \\
\text { River tributary }\end{array}$ & $\begin{array}{l}\text { NE } 1 / 2 \text { NE } 1 / \text { sec. } 32, T .29 \text { N., R. } 17 \text { W., } \\
\text { St. Croix County, at bridge on } \\
\text { country road, } 1.5 \text { mi southwest } \\
\text { of Hammond, Wis. }\end{array}$ & 1.88 & $\begin{array}{l}\text { Aug. } 16,1972 \\
\text { Oct. 18, } 1973 \\
\text { July } 29,1975 \\
\text { June } 8,1976\end{array}$ & $\begin{array}{l}2 \\
2_{0}^{0} \\
2_{0}^{0} \\
{ }^{0} 0\end{array}$ & 0 & $\mathbf{0}$ & c \\
\hline SC1B & Hatchery Creek & $\begin{array}{l}\text { NE } 1 / 4 \text { NE } 1 / \text { sec. } 26, T .41 \text { N., R. } 9 \text { W., } \\
\text { Sawyer County, at culverts on } \\
\text { country road, } 1.6 \text { mi east of } \\
\text { Hayward, Wis. }\end{array}$ & 2.53 & $\begin{array}{l}\text { Aug. } 12,1975 \\
\text { June } 8,1976 \\
\text { Aug. } 4,1976 \\
\text { Sept. 30, } 1976 \\
\text { Aug. } 25,1977\end{array}$ & $\begin{array}{l}1.53 \\
1.75 \\
1.64 \\
1.64 \\
1.79\end{array}$ & 1.6 & 1.3 & c \\
\hline$S C 2 B$ & Godfrey Creek & $\begin{array}{l}\text { NW1/4NW1/4 sec. 12, T. } 39 \text { N., R. } 10 \text { W., } \\
\text { Washburn County, at culverts on } \\
\text { Lake Road, } 2.7 \text { mi northwest of } \\
\text { Stone Lake, Wis. }\end{array}$ & 2.44 & $\begin{array}{l}\text { Aug. } 11,1975 \\
\text { June } 8,1976 \\
\text { Aug. } 5,1976 \\
\text { Sapt. 30, 1976 } \\
\text { Aug. 25, } 1977\end{array}$ & $\begin{array}{l}.54 \\
.61 \\
.66 \\
.66 \\
.57\end{array}$ & .61 & .51 & c \\
\hline $\operatorname{sc3B}$ & $\begin{array}{l}\text { St. Croix } \\
\text { River tributary }\end{array}$ & $\begin{array}{l}\text { SW } 1 / 2 N E 1 / 4 \text { sec. } 14, T .33 \text { N., R. } 19 \text { W., } \\
\text { Polk County, at road to fish } \\
\text { hatchery, } 2.1 \text { mi northeast of } \\
\text { Osceola, Wis. }\end{array}$ & 0.38 & $\begin{array}{l}\text { June } 9,1976 \\
\text { Aug. 3, } 1976 \\
\text { Sept. 28, } 1976\end{array}$ & $\begin{array}{l}4.33 \\
3.49 \\
4.35\end{array}$ & 2.1 & .89 & $\mathbf{c}$ \\
\hline
\end{tabular}

1 Based on 1972 report by Wisconsin Department of Natural Resources (DNR). Site lettered "B" was not included in 1972 DNR report.

2 Streambed is dry.

${ }^{4}$ Negligible discharge--water ponded, unable to measure velocity. 
Table 23. Sheboygan River basin station location and low-flow characteristics.

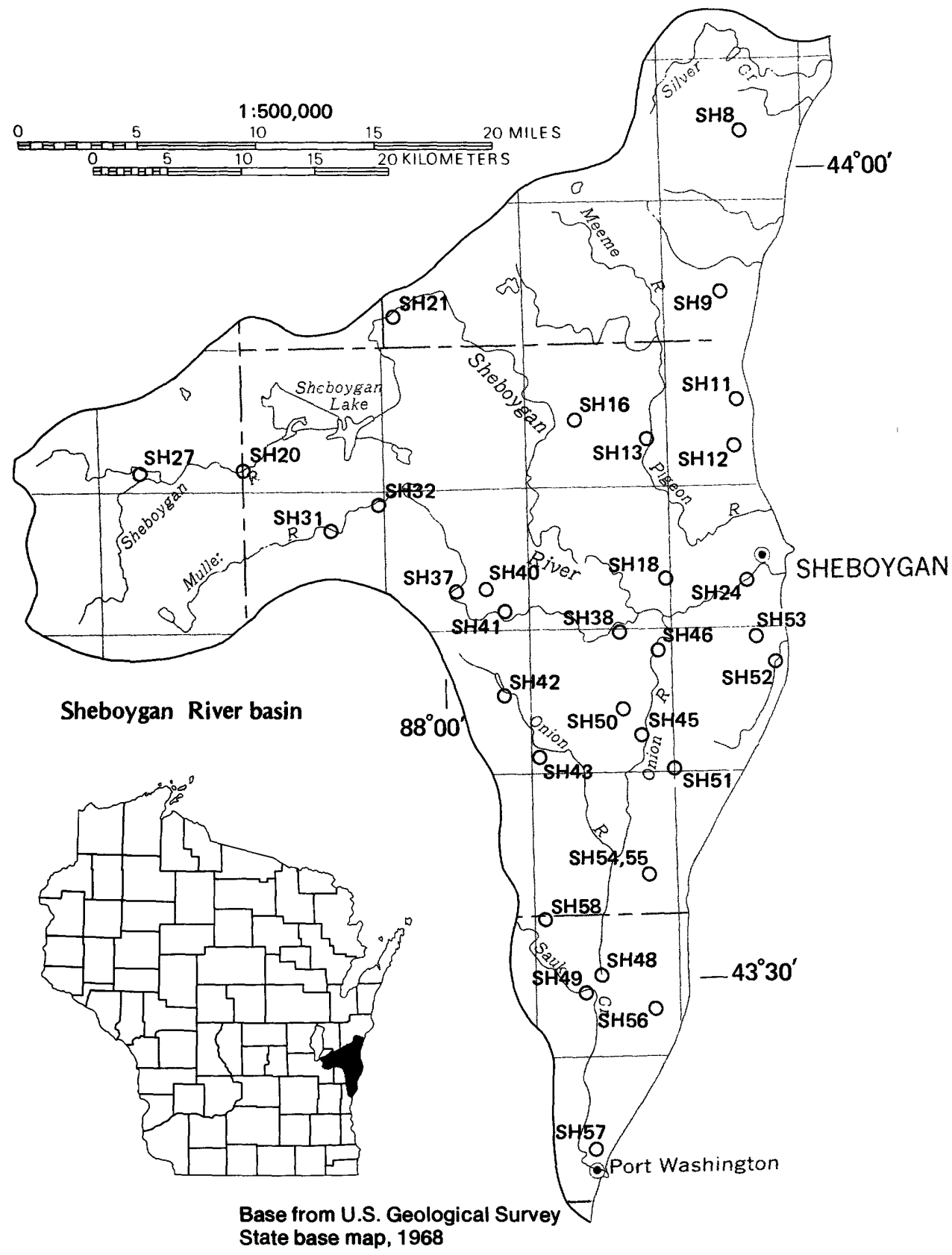

\begin{tabular}{|c|c|c|c|c|c|c|}
\hline $\begin{array}{l}\text { Station }{ }^{1} \\
\text { number }\end{array}$ & $\underset{\text { name }}{\text { Stream }}$ & Station location & $\begin{array}{l}\text { Drainage } \\
\text { area } \\
\left(\mathrm{mi}^{2}\right)\end{array}$ & Date & $\begin{array}{c}\text { Discharge } \\
\left(\mathrm{ft}^{3} / \mathrm{s}\right)\end{array}$ & 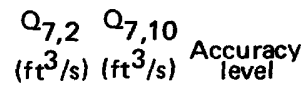 \\
\hline
\end{tabular}

SH8 Pine Creek

Pine Creek tributary
SE $1 / 4 S^{1} / \frac{\text { sec. } 22, T .}{18}$ N., R. 23 E. Manitowoc County, at bridge on County Trunk C, 1.0 mi northeast of Newton, Wis.

NW\% 14 SW1/4 sec. 27, T. 18 N., R. 23 E. Manitowoc County, at Stock's Supper Club end manufacturing company sewage-treatment plant, $0.7 \mathrm{mi}$ northwest of Newton, Wis.
6.17

July 17,1972

July 12, 1973

0.30

1.06

Aug 21, 1973

Sept. 24, 1974

Aug. 14, 1975

Aug. 12, 1976

1.07 
Table 23. Sheboygan River basin station location and low-flow characteristics-Continued.

\begin{tabular}{|c|c|c|c|c|c|c|c|c|}
\hline $\begin{array}{l}\text { Station } \\
\text { number }\end{array}$ & 1 Stream & Station location & $\begin{array}{l}\text { Drainage } \\
\text { areag } \\
\left(\mathrm{mi}^{2}\right)\end{array}$ & Date & $\begin{array}{c}\text { Discharge } \\
\left(\mathrm{ft}^{3} / \mathrm{s}\right)\end{array}$ & $\begin{array}{l}a_{7,2} a_{7} \\
\left(\mathrm{ft}^{3} / \mathrm{s}\right)\left(\mathrm{ft}^{3}\right.\end{array}$ & & $\begin{array}{c}\text { Accuracy } \\
\text { level }\end{array}$ \\
\hline SH9 & Centerville Creek & $\begin{array}{l}\text { NE } 1 / 4 \text { SE } / 4 \text { sec. } 21, T .17 \text { N., R. } 23 \text { E., } \\
\text { Manitowoc County, } 0.8 \text { mi north } \\
\text { of State Highway } 149, \text { at Chicago } \\
\text { and NorthWestern Railroad, at } \\
\text { Cleveland, Wis. }\end{array}$ & 3.15 & $\begin{array}{l}\text { Sept. } 24,1974 \\
\text { Aug. } 13,1975 \\
\text { Aug. 12, } 1976 \\
\text { Sept. 29, } 1976 \\
\text { July } 13,1977\end{array}$ & $\begin{array}{l}2.08 \\
<01 \\
.01 \\
.12 \\
.13\end{array}$ & 0 & 0 & c \\
\hline SH11 & Sevenmile Creek & $\begin{array}{l}\text { NE } 1 / 4 \text { SE } 1 / 4 \text { sec. } 16, T .16 \text { N., R. } 23 \text { E., } \\
\text { Sheboygan County, at bridge on } \\
\text { County Trunk LS, } 0.4 \text { mi northeast } \\
\text { of Haven, Wis. }\end{array}$ & 5.57 & $\begin{array}{l}\text { July } 19,1972 \\
\text { July } 12,1973 \\
\text { Aug. } 21,1973 \\
\text { Sept. } 24,1974 \\
\text { Aug. } 13,1975 \\
\text { Aug. } 12,1976\end{array}$ & $\begin{array}{l}.16 \\
.35 \\
.55 \\
.14 \\
.04\end{array}$ & 0 & 0 & c \\
\hline $\mathrm{SH} 12$ & $\begin{array}{l}\text { Lake Michigan } \\
\text { tributary }\end{array}$ & $\begin{array}{l}\text { SEY } / 4 S E 1 / 4 \text { sec. } 28, T .16 \text { N., R. } 23 \text { E., } \\
\text { Sheboygan County, at bridge on } \\
\text { County Trunk LS, } 3.0 \text { mi northwest } \\
\text { of post office, at Sheboygan, Wis. }\end{array}$ & .87 & $\begin{array}{l}\text { Sept. } 24,1974 \\
\text { Aug. } 13,1975 \\
\text { Aug. } 12,1976 \\
\text { Sept. 29, } 1976\end{array}$ & $\begin{array}{l}2 \\
20 \\
2 \\
2 \\
2\end{array}$ & 0 & 0 & c \\
\hline SH13 & Pigeon River & $\begin{array}{l}\text { SEY//SE } 1 / 4 \text { sec. } 26, T .16 \text { N., R. } 22 \text { E., } \\
\text { Sheboygen County, at sewage- } \\
\text { treatment plant, } 0.2 \text { mi east of } \\
\text { State Highway } 32,0.3 \text { mi northeast } \\
\text { of Millersville, Wis. }\end{array}$ & 50.5 & $\begin{array}{l}\text { July } 19,1972 \\
\text { July } 12,1973 \\
\text { Aug. } 21,1973 \\
\text { Sept. } 24,1974 \\
\text { Aug. } 13,1975 \\
\text { Aug. } 12,1976\end{array}$ & $\begin{array}{l}3.97 \\
5.31 \\
23.1 \\
4.68 \\
1.68 \\
3.14\end{array}$ & .21 & .02 & c \\
\hline SH16 & $\begin{array}{l}\text { Pigeon River } \\
\text { tributary }\end{array}$ & $\begin{array}{l}\text { SE } 1 / 4 \text { SW } 1 / 4 \text { sec. } 21, T .16 \text { N., R. } 22 \text { E., } \\
\text { Sheboygan County, at bridge on } \\
\text { County Trunk A, } 1.3 \text { mi east of } \\
\text { Franklin, Wis. }\end{array}$ & 1.38 & $\begin{array}{l}\text { July } 19,1972 \\
\text { July } 12,1973 \\
\text { Aug. } 12,1973 \\
\text { Sept. } 24,1974 \\
\text { Aug. } 13,1975 \\
\text { Aug. } 12,1976\end{array}$ & $\begin{array}{l}.07 \\
.03 \\
.06 \\
.04 \\
.03 \\
.08\end{array}$ & $<.01$ & $<.01$ & c \\
\hline SH18 & $\begin{array}{l}\text { Pigeon River } \\
\text { tributary }\end{array}$ & $\begin{array}{l}\text { SE } 1 / \text { SE } 1 / 4 \text { sec. } 13, T .15 \mathrm{~N} ., \mathrm{R} .22 \mathrm{E} \text {., } \\
\text { Sheboygan County, at bridga on } \\
\text { country road, } 0.2 \text { mi north of } \\
\text { County Trunk } 0,1.5 \text { mi northwest } \\
\text { of Kohler, Wis. }\end{array}$ & 7.32 & $\begin{array}{l}\text { July } 18,1972 \\
\text { July } 12,1973 \\
\text { Aug. } 21,1973 \\
\text { Sept. } 24,1974 \\
\text { Aug. } 12,1975\end{array}$ & $\begin{array}{l}4.02 \\
40 \\
20 \\
20\end{array}$ & 0 & 0 & c \\
\hline SH2O & Sheboygan River & $\begin{array}{l}\text { SE1/2NE1/4 sec. } 36, \text { T. } 16 \text { N., R. } 19 \text { E., } \\
\text { Fond du Lac County, at bridge on } \\
\text { local road, } 0.1 \text { mi east of County } \\
\text { Trunk G, } 0.5 \text { mi south of St. Cloud, } \\
\text { Wis. }\end{array}$ & 56.3 & $\begin{array}{l}\text { July } 18,1972 \\
\text { July } 13,1973 \\
\text { Nov. } 15,1973 \\
\text { Sept. } 25,1974 \\
\text { Aug. } 11,1975 \\
\text { Aug. } 11,1976\end{array}$ & $\begin{array}{c}5.43 \\
9.17 \\
35.4 \\
3.94 \\
.77 \\
1.13\end{array}$ & .40 & .12 & c \\
\hline SH21 & Sheboygan River & $\begin{array}{l}\text { NW/4SEY/4 sec. 30, T. } 17 \text { N., R. } 21 \text { E., } \\
\text { Manitowoc County, at bridge on } \\
\text { State Highway 32, at Kiel, Wis. }\end{array}$ & 136 & $\begin{array}{l}\text { July } 18,1972 \\
\text { July } 12,1973 \\
\text { Aug. } 20,1973 \\
\text { Sept. } 24,1974 \\
\text { Aug. } 14,1975 \\
\text { Aug. } 11,1976\end{array}$ & $\begin{array}{l}14.7 \\
.63 \\
6.60 \\
4.67 \\
1.99 \\
2.27\end{array}$ & 1.5 & .72 & c \\
\hline SH24 & Sheboygan River & $\begin{array}{l}\text { SE1/1/4E1/4 sec. 29, T. } 15 \mathrm{~N} ., \text { R. } 23 \text { E., } \\
\text { Sheboygan County, } 0.7 \mathrm{mi} \text { upstream } \\
\text { from State Highway } 28,2.7 \mathrm{mi} \\
\text { southwest of downtown Sheboygan, Wis. }\end{array}$ & 432 & & & 28 & 16 & $\mathbf{a}$ \\
\hline SH27 & $\begin{array}{l}\text { Sheboygan River } \\
\text { tributary }\end{array}$ & $\begin{array}{l}\text { NE } 1 / 4 \text { NW1/ sec. } 32, \text { T. } 16 \text { N., R. } 19 \text { E., } \\
\text { Fond du Lac. County, at bridge on } \\
\text { County Trunk W, at Mt. Calvary. } \\
\text { Wis. }\end{array}$ & 13.1 & $\begin{array}{l}\text { July } 19,1972 \\
\text { July } 13,1973 \\
\text { Nov. } 15,1973 \\
\text { Sept. } 25,1974 \\
\text { Aug. } 11,1975\end{array}$ & $\begin{array}{l}{ }_{4}^{4} 0 \\
0 \\
3.95 \\
.18 \\
.20\end{array}$ & 0 & 0 & c \\
\hline SH31 & Mullet River & $\begin{array}{l}\text { SE1/4SE1/4 sec. } 10, \mathrm{~T} .15 \mathrm{~N} . \text {, R. } 20 \mathrm{E} \text {., } \\
\text { Sheboygan County, at bridga on } \\
\text { State Highwey } 23 \text {, at Greenbush, Wis. }\end{array}$ & 50.8 & $\begin{array}{l}\text { July } 18,1972 \\
\text { July } 13,1973 \\
\text { Aug. } 20,1973 \\
\text { Sept. } 25,1974 \\
\text { Aug. } 11,1975 \\
\text { Aug. } 11,1976 \\
\text { July } 12,1977\end{array}$ & $\begin{array}{c}1.61 \\
1.56 \\
10.2 \\
1.49 \\
1.15 \\
1.26 \\
2.23\end{array}$ & .51 & .30 & c \\
\hline
\end{tabular}

Mullet River tributary
SE $1 / 4$ SE $1 / 4$ sec. 10, T. 15 N., R. 20 E., Sheboygan County, at State Highway 22, at Greenbush, Wis. 
Table 23. Sheboygan River basin station location and low-flow characteristics-Continued.

\begin{tabular}{|c|c|c|c|c|c|c|c|c|}
\hline $\begin{array}{l}\text { Station } \\
\text { number }\end{array}$ & Stream & Station location & $\begin{array}{l}\text { Drainage } \\
\text { area } \\
\left(\mathbf{m i}^{2}\right)\end{array}$ & Date & $\begin{array}{c}\text { Discharge } \\
\left(\mathrm{ft}^{3} / \mathrm{s}\right)\end{array}$ & $\begin{array}{l}\mathrm{Q}_{7,2} \mathrm{Q}_{7,} \\
\left(\mathrm{ft}^{3} / \mathrm{s}\right)\left(\mathrm{ft}^{3}\right)\end{array}$ & $10 \mathrm{Ac}$ & $\begin{array}{l}\text { Accuracy } \\
\text { level }\end{array}$ \\
\hline SH32 & Mullet River & $\begin{array}{l}\text { SE1/aNE1/4 sec. 1, T. } 15 \text { N., R. } 20 \text { E., } \\
\text { Sheboygan River, at bridge on } \\
\text { County Trunk P. at Glenbeulah, Wis. }\end{array}$ & 55.7 & $\begin{array}{l}\text { Oct. } 4,1968 \\
\text { July } 18 ., 1972 \\
\text { July } 13,1973 \\
\text { Aug. } 20,1973 \\
\text { Sept. 25, 1974 } \\
\text { Aug. } 11,1975 \\
\text { Aug. } 11,1976\end{array}$ & $\begin{array}{l}0.80 \\
1.90 \\
1.65 \\
12.5 \\
3.86 \\
1.69 \\
3.55\end{array}$ & 0.80 & 0.32 & c \\
\hline SH37 & Mullet River & $\begin{array}{l}\text { SEY/4SW1/4 sec. } 27, \text { T. } 15 \text { N., R. } 21 \text { E., } \\
\text { Sheboygan County, at bridge on } \\
\text { County Trunk PP, } 1.0 \text { mi south of } \\
\text { State Highway } 23, \text { in Plymouth, Wis. }\end{array}$ & 85.0 & $\begin{array}{lc}\text { Oct. } & 3,1968 \\
\text { July } 18,1972 \\
\text { July } 11,1973 \\
\text { Aug. } 20,1973 \\
\text { Sept. } 24,1974 \\
\text { Aug. } 12,1975 \\
\text { Aug. } 11,1976\end{array}$ & $\begin{array}{l}8.72 \\
12.7 \\
21.4 \\
31.2 \\
18.9 \\
14.4 \\
14.0\end{array}$ & 6.2 & 2.9 & c \\
\hline SH38 & Mullet River & $\begin{array}{l}\text { NW1/4SE } 1 / 4 \text { sec. } 35, \text { T. } 15 \text { N., R. } 22 \text { E., } \\
\text { Sheboygan County, at bridge on } \\
\text { County Trunk PP, on southwest side } \\
\text { of Sheboygan Falis, Wis. }\end{array}$ & 108 & $\begin{array}{l}\text { July } 19,1972 \\
\text { July } 11,1973 \\
\text { Aug. } 21,1973 \\
\text { Sept. } 24,1974 \\
\text { Aug. } 13,1975 \\
\text { Aug. } 11,1976\end{array}$ & $\begin{array}{l}15.0 \\
23.4 \\
36.0 \\
18.9 \\
13.9 \\
24.9\end{array}$ & 9.8 & 5.6 & c \\
\hline \multirow[t]{2}{*}{ SH4O } & $\begin{array}{l}\text { Mullet River } \\
\text { tributary }\end{array}$ & $\begin{array}{l}\text { NE } 1 / 4 S^{1} / 4 \text { sec. } 26, T .15 \text { N., R. } 21 \text { E., } \\
\text { Sheboygan County, at La Graves trailer } \\
\text { park septic tank, just west of railroad } \\
\text { crossing of State Highway } 57,1.0 \mathrm{mi} \\
\text { east of Plymouth, Wis. }\end{array}$ & .64 & & & & & \\
\hline & $\begin{array}{l}\text { Mullet River } \\
\text { tributary }\end{array}$ & $\begin{array}{l}\text { SW } 1 / 4 \text { SW } 1 / 4 \text { see. } 26, T .15 \text { N., R. } 21 \text { E., } \\
\text { Sheboygen County, at culvert under } \\
\text { County Trunk PP, } 1.5 \text { mi southeast } \\
\text { of Plymouth, Wis. }\end{array}$ & 1.33 & $\begin{array}{l}\text { July } 19,1972 \\
\text { July } 11,1973 \\
\text { Aug. } 20,1973 \\
\text { Sept. 24, 1974 } \\
\text { Aug. } 12,1975 \\
\text { Aug. } 11,1975\end{array}$ & $\begin{array}{l}.19 \\
.47 \\
.04 \\
.16 \\
.10 \\
.07\end{array}$ & .05 & .02 & c \\
\hline SH41 & $\begin{array}{l}\text { Mullet River } \\
\text { tributary }\end{array}$ & $\begin{array}{l}\text { SE1/4SW1/4 sec. 25, T. } 15 \text { N., R. } 21 \text { E., } \\
\text { Sheboygan County, at Gilson Manu- } \\
\text { facturing Company, just northwest of } \\
\text { junction of State Highway } 57 \text { and County } \\
\text { Trunk PP, } 2.5 \text { mi southeast of Plymouth, } \\
\text { Wis. }\end{array}$ & y & July 18, 1972 & ${ }^{2} 0$ & 0 & 0 & c \\
\hline \multirow[t]{2}{*}{ SH42 } & Onion River & $\begin{array}{l}\text { NE1/4SE1/4 sec. 10, T. } 14 \text { N., R. } 21 \text { E., } \\
\text { Sheboygan County, at County Trunk } \\
\text { AC, } 1.4 \text { mi northwest of Waldo, Wis. }\end{array}$ & 18.2 & $\begin{array}{l}\text { July } 20,1972 \\
\text { July } 11,1973 \\
\text { Aug. } 20,1973 \\
\text { Nov. 13,1973 } \\
\text { Sept. 24, } 1974 \\
\text { Aug. } 12,1975 \\
\text { Aug. 10, } 1976 \\
\text { Sept. 29, } 1976 \\
\text { July } 12,1977\end{array}$ & $\begin{array}{c}39.36 \\
10.7 \\
14.0 \\
18.6 \\
14.3 \\
10.7 \\
9.29 \\
9.17 \\
11.3\end{array}$ & 6.2 & 4.0 & b \\
\hline & Onion River & $\begin{array}{l}\text { SW1/4SW1/4 sec. 13, T. } 14 \text { N., R. } 21 \text { E., } \\
\text { Sheboygan County, at sewage-treatment } \\
\text { plant, } 1.0 \text { mi east of Waldo, Wis. }\end{array}$ & 24.3 & & & & & \\
\hline SH43 & Onion River & $\begin{array}{l}\text { NW1/4NW1/4 sec. } 31, \text { T. } 14 \text { N., R. } 22 \text { E., } \\
\text { Sheboygan County, at bridge on } \\
\text { County Trunk I, at Hingham, Wis. }\end{array}$ & 33.7 & $\begin{array}{l}\text { July } 20,1972 \\
\text { July } 11,1973 \\
\text { Aug. } 21,1973 \\
\text { Sept. 24, 1974 } \\
\text { Aug. } 12,1975 \\
\text { Aug. 10,1976 } \\
\text { July } 12,1977\end{array}$ & $\begin{array}{c}6.77 \\
8.35 \\
7.03 \\
11.6 \\
5.62 \\
9.06 \\
8.51\end{array}$ & 4.7 & 3.2 & c \\
\hline SH45 & Onion River & $\begin{array}{l}\text { NE1/4SE } 1 / 1 \text { sec. } 26, \text { T. } 14 \text { N., R. } 22 \text { E., } \\
\text { Sheboygan County, at bridge on } \\
\text { County Trunk OO, just east of } \\
\text { Gibbsville, Wis. }\end{array}$ & 79.5 & $\begin{array}{l}\text { July } 20,1972 \\
\text { July } 12,1973 \\
\text { Aug. } 21,1973 \\
\text { Sept. 24, 1974 } \\
\text { Aug. } 13,1975 \\
\text { Aug. 10, } 1976 \\
\text { July 12, } 1977\end{array}$ & $\begin{array}{l}20.6 \\
11,3 \\
13.0 \\
13.8 \\
12.2 \\
10.6 \\
8.38\end{array}$ & 7.2 & 4.7 & c \\
\hline
\end{tabular}


Table 23. Sheboygan River basin station location and low-flow characteristics-Continued.

\begin{tabular}{|c|c|c|c|c|c|c|c|c|}
\hline $\begin{array}{l}\text { Station } \\
\text { number }\end{array}$ & Stream & Station location & $\begin{array}{l}\text { Drainage } \\
\text { area } \\
\left(\mathrm{mi}^{2}\right)\end{array}$ & Date & $\begin{array}{c}\text { Discharge } \\
\left(\mathrm{ft}^{3} / \mathrm{s}\right)\end{array}$ & $\begin{array}{l}\mathrm{Q}_{7,2} \mathrm{Q}_{7} \\
\left(\mathrm{ft}^{3} / \mathrm{s}\right)\left(\mathrm{ft}^{3}\right.\end{array}$ & & $\begin{array}{c}\text { Accuracy } \\
\text { level }\end{array}$ \\
\hline SH46 & Onion River & $\begin{array}{l}\text { SW } 1 / 4 \text { NW } 1 / 4 \text { sec. } 12, T .14 \text { N., R. } 22 \text { E., } \\
\text { Sheboygan County, at country road } \\
\text { bridge, } 2.3 \text { mi south of Sheboygan } \\
\text { Falls, Wis. }\end{array}$ & 91.8 & $\begin{array}{l}\text { July } 20,1972 \\
\text { July } 12,1973 \\
\text { Aug. } 21,1973 \\
\text { Sept. } 24,1974 \\
\text { Aug. } 13,1975 \\
\text { Aug. } 10,1976 \\
\text { July } 12,1977\end{array}$ & $\begin{array}{l}17.0 \\
8.45 \\
19.5 \\
18.7 \\
5.58 \\
9.15 \\
8.64\end{array}$ & 6.7 & 4.4 & c \\
\hline SH48 & $\begin{array}{l}\text { Tributary to } \\
\text { Onion River } \\
\text { tributary }\end{array}$ & $\begin{array}{l}\text { SW } 1 / 4 \text { SE } 1 / 4 \text { sec. 15, T. } 12 \text { N., R. } 22 \text { E., } \\
\text { Ozaukee County, at culvert on } \\
\text { Park Street, at Belgium, Wis. }\end{array}$ & .16 & $\begin{array}{l}\text { Sept. } 23,1974 \\
\text { Aug. } 12,1975 \\
\text { Aug. } 9,1976 \\
\text { Sept. } 29,1976 \\
\text { July } 11,1977\end{array}$ & $\begin{array}{l}.89 \\
.50 \\
.23 \\
.17 \\
.12\end{array}$ & $\underline{5}$ & $\underline{\mathbf{5}}$ & \\
\hline SH49 & Sauk Creek & $\begin{array}{l}\text { SW } 1 / 4 \text { SEY sec. } 21, T .12 \text { N., R. } 22 \text { E., } \\
\text { Ozaukee County, at bridge on country } \\
\text { road, } 1.5 \text { mi southwest of Belgium, Wis. }\end{array}$ & 19.3 & $\begin{array}{l}\text { July } 10,1973 \\
\text { Aug. } 22,1973 \\
\text { Nov. } 13,1973 \\
\text { Sept. 23, } 1974 \\
\text { Aug. } 12,1975 \\
\text { Aug. } 9,1976\end{array}$ & $\begin{array}{l}0.60 \\
.60 \\
4.80 \\
.15 \\
2.01 \\
0\end{array}$ & 0 & 0 & c \\
\hline \multirow[t]{2}{*}{ SH5O } & $\begin{array}{l}\text { Onion River } \\
\text { tributary }\end{array}$ & $\begin{array}{l}\text { NE1/4SE1/4 sec. 17, T. } 14 \text { N., R. } 22 \text { E., } \\
\text { Sheboygan County, at Sheboygan } \\
\text { County Hospital sewage-treatment } \\
\text { plant, } 3.3 \text { mi northwest of } \\
\text { Gibbsville, Wis. }\end{array}$ & 1.31 & & & & & \\
\hline & $\begin{array}{l}\text { Onion River } \\
\text { tributary }\end{array}$ & $\begin{array}{l}\text { NE1/4SE1/4 sec. 16, T. } 14 \text { N., R. } 21 \text { E., } \\
\text { Sheboygan County, at bridge on } \\
\text { County Trunk 1, } 2.6 \text { mi northwest of } \\
\text { Gibbsville, Wis. }\end{array}$ & 1.92 & $\begin{array}{l}\text { July } 20,1972 \\
\text { July } 12,1973 \\
\text { Aug. } 21,1973 \\
\text { Aug. } 13,1975 \\
\text { Aug. } 10,1976\end{array}$ & $\begin{array}{l}.09 \\
.03 \\
.12 \\
.02 \\
.04\end{array}$ & .01 & $<.01$ & c \\
\hline SH51 & Black River & $\begin{array}{l}\text { NE } 1 / 4 N E 1 / 4 \text { sec. } 1, T .13 \text { N., R. } 22 \text { E., } \\
\text { Sheboygan County, } 0.1 \text { mi west of } \\
\text { County Trunk A, } 0.5 \text { mi north of } \\
\text { Oostburg, Wis. }\end{array}$ & .49 & $\begin{array}{l}\text { July } 11,1973 \\
\text { Aug. } 21,1973 \\
\text { Sept. } 24,1974 \\
\text { Aug. } 13,1975 \\
\text { Aug. } 10,1976\end{array}$ & $\begin{array}{l}20 \\
20 \\
20 \\
20 \\
20\end{array}$ & 0 & 0 & c \\
\hline SH52 & Black River & $\begin{array}{l}\text { NW1/4NEY// sec. 11, T. } 14 \text { N., R. } 23 \text { E., } \\
\text { Sheboygan County, at bridge on } \\
\text { country road, } 2.9 \text { mi southeast } \\
\text { of State Highway } 28 \text { and U.S. } \\
\text { Highway } 141 \text { intersection in } \\
\text { Sheboygan, Wis. }\end{array}$ & 15.7 & $\begin{array}{l}\text { Aug. } 20,1972 \\
\text { July } 11,1973 \\
\text { Aug. } 21,1973 \\
\text { Sept. } 24,1974 \\
\text { Aug. } 13,1975 \\
\text { Aug. } 10,1976\end{array}$ & $\begin{array}{l}{ }_{4}^{4} \\
{ }^{4} \\
4_{0}^{4} \\
{ }_{4}^{0} \\
{ }_{4}^{0}\end{array}$ & 0 & 0 & c \\
\hline SH53 & $\begin{array}{l}\text { Hartman's } \\
\text { Creek }\end{array}$ & $\begin{array}{l}\text { SE } 1 / 4 N E 1 / 4 \text { sec. } 10, T .14 \mathrm{~N} ., \mathrm{R} .22 \mathrm{E} . \text {, } \\
\text { Sheboygan County, at bridge on } \\
\text { County Trunk KK, } 3.3 \mathrm{mi} \text { south of } \\
\text { State Highway } 28 \text { and U.S. } \\
\text { Highway } 141 \text { intersection in } \\
\text { Sheboygan, Wis. }\end{array}$ & 1.61 & $\begin{array}{l}\text { July } 20,1972 \\
\text { July } 11,1973 \\
\text { Aug. } 21,1973 \\
\text { Nov. } 13,1973 \\
\text { Sept. } 24,1974 \\
\text { Aug. } 13,1975 \\
\text { Aug. } 10,1976\end{array}$ & $\begin{array}{l}.17 \\
.03 \\
.12 \\
.08 \\
.08 \\
.02 \\
.09\end{array}$ & .01 & $<.01$ & c \\
\hline$\underset{55}{\text { SH54, }}$ & $\begin{array}{l}\text { Barr Creek } \\
\text { tributary }\end{array}$ & $\begin{array}{l}\text { NE } 1 / 4 \text { SW } 1 / 4 \text { sec. } 24, T .13 \text { N., R. } 23 \text { E., } \\
\text { Sheboygan County, at sewage-treatment } \\
\text { plant, } 0.7 \text { mi northeast of Cedar } \\
\text { Grove, Wis. }\end{array}$ & .94 & $\begin{array}{l}\text { July } 10,1973 \\
\text { Aug. } 22,1973 \\
\text { Sept. } 23,1974 \\
\text { Aug. } 12,1975\end{array}$ & $\begin{array}{l}.44 \\
.22 \\
.40 \\
.34\end{array}$ & $\underline{5}$ & $\underline{5}$ & \\
\hline SH56 & Sucker Creek & $\begin{array}{l}\text { NE } 1 / 4 N E 1 / 4 \text { sec. } 23, \text { T. } 12 \text { N., R. } 22 \text { E., } \\
\text { Ozaukee County, at bridge on County } \\
\text { Trunk D, at Lake Church, Wis. }\end{array}$ & 5.01 & & & & & \\
\hline & Sucker Creek & $\begin{array}{l}\text { SE } 1 / 4 \text { SE } 1 / 4 \text { sec. } 23, T .12 \text { N., R. } 22 \text { E., } \\
\text { Ozaukee County, at bridge on country } \\
\text { road, } 1.0 \text { mi south of Lake Church, Wis. }\end{array}$ & 6.25 & $\begin{array}{l}\text { July } 17,1972 \\
\text { July } 10,1973 \\
\text { Aug. } 22,1973 \\
\text { Sept. 23, } 1974 \\
\text { Aug. } 12,1975 \\
\text { Aug. } 9,1976\end{array}$ & $\begin{array}{l}.72 \\
.12 \\
.07 \\
.09 \\
.02 \\
.04\end{array}$ & $<.01$ & $<.01$ & c \\
\hline
\end{tabular}


Table 23. Sheboygan River basin station location and low-flow characteristics-Continued.

\begin{tabular}{|c|c|c|c|c|c|c|c|}
\hline $\begin{array}{l}\text { Station } \\
\text { number }\end{array}$ & 1 Stream & Station location & $\begin{array}{c}\text { Drainage } \\
\text { area } \\
\left(\mathrm{mi}^{2}\right)\end{array}$ & Date & $\begin{array}{c}\text { Discharge } \\
\left(\mathrm{ft}^{3} / \mathrm{s}\right)\end{array}$ & $\begin{array}{cc}a_{7,2} & a_{7,10} \\
\left(\mathrm{ft}^{3} / \mathrm{s}\right) & \left(\mathrm{ft}^{3} / \mathrm{s}\right)\end{array}$ & $\begin{array}{c}\text { Accuracy } \\
\text { level }\end{array}$ \\
\hline \multirow[t]{2}{*}{ SH57 } & $\begin{array}{l}\text { Lake Michigan } \\
\text { tributary }\end{array}$ & $\begin{array}{l}\text { NE1/4SE1/4 sec. 16, T. } 11 \text { N., R. } 22 \text { E., } \\
\text { Ozaukee County, at intersection of } \\
\text { State Highway } 32 \text { and country road, } \\
0.5 \text { mi northeast of Knellsvilla, Wis. }\end{array}$ & 1.05 & & & & \\
\hline & $\begin{array}{l}\text { Lake Michigan } \\
\text { tributary }\end{array}$ & $\begin{array}{l}\text { SEY/4NEY/4 sec. } 21, T .11 \mathrm{~N} ., \mathrm{R} .22 \mathrm{E} . \text {, } \\
\text { Ozaukea County, at country road } \\
\text { bridge, } 0.7 \mathrm{mi} \text { southeast of } \\
\text { Knellsville, Wis. }\end{array}$ & 1.72 & $\begin{array}{l}\text { July } 17,1972 \\
\text { July } 10,1973 \\
\text { Aug. } 23,1973 \\
\text { Sept. } 23,1974 \\
\text { Aug. } 12,1975 \\
\text { Aug. } 9,1976\end{array}$ & $\begin{array}{l}.08 \\
.04 \\
.01 \\
.01 \\
.05 \\
<.01\end{array}$ & $<.01<.01$ & c \\
\hline SH58 & $\begin{array}{l}\text { Sauk Creak } \\
\text { tributary }\end{array}$ & $\begin{array}{l}\text { NE } 1 / 4 \text { SE } 1 / 4 \text { sec. } 1, T .12 \text { N., R. } 21 \text { E., } \\
\text { Ozaukee County, at culvert on } \\
\text { country road, } 2.4 \text { mi southeast of } \\
\text { Random Lake, Wis. }\end{array}$ & 2.19 & $\begin{array}{l}\text { Sept. 23, } 1974 \\
\text { Aug. 12, } 1975 \\
\text { Aug. 10, } 1976 \\
\text { Sept. 29, } 1976 \\
\text { July 11, } 1977\end{array}$ & $\begin{array}{l}.25 \\
.13 \\
.11 \\
.06 \\
.05\end{array}$ & $.02<.01$ & c \\
\hline
\end{tabular}

\footnotetext{
1 Based on 1970 report by Wisconsin Department of Natural Resources.

${ }^{2}$ Streambed is dry.

3 Additional discharge measurements are available.

${ }^{4}$ Negligible discharge--water ponded, unable to measure velocity.

${ }^{5}$ No estimate possible--discharge is primarily effluent.
} 
Table 24. Sugar River basin station locations and low-flow characteristics.
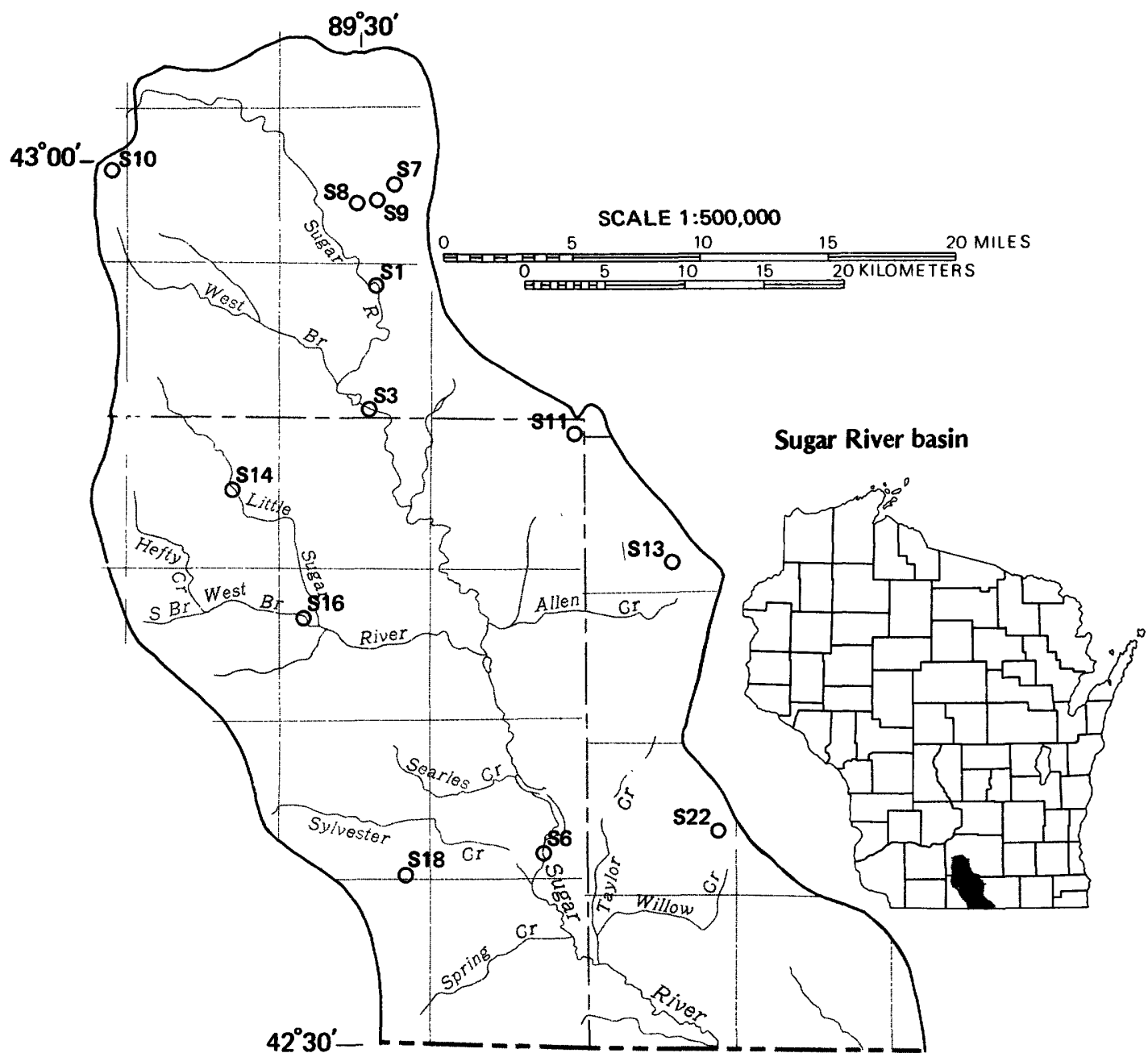

Base from U.S. Geological Survey

State base map, 1968

\begin{tabular}{|c|c|c|c|c|c|c|c|c|}
\hline $\begin{array}{l}\text { Station } \\
\text { number }\end{array}$ & $r$ Stream & Station location & $\begin{array}{l}\text { Drainage } \\
\text { area } \\
\left(\mathrm{mi}^{2}\right)\end{array}$ & Date & $\begin{array}{c}\text { Discharge } \\
\left(\mathrm{ft}^{3} / \mathrm{s}\right)\end{array}$ & $\begin{array}{l}Q_{7,2} Q \\
\left(\mathrm{ft}^{3} / \mathrm{s}\right)(\mathrm{f}\end{array}$ & $\begin{array}{l}7,10 \\
\left.f^{3} / s\right)\end{array}$ & $\begin{array}{l}\text { Iccuracy } \\
\text { level }\end{array}$ \\
\hline S1 & Sugar River & $\begin{array}{l}\text { NE } 1 / 4 \text { SEY } 1 / 4 \text { sec. } 3, \text { T. } 5 \text { N., R. } 8 \text { E., } \\
\text { Dane County, at bridge on country } \\
\text { road, } 0.3 \text { mi north of Paoli, Wis. }\end{array}$ & 88.7 & $\begin{array}{l}\text { Nov. } 6,1975 \\
\text { July } 26,1976 \\
\text { Aug. } 3,1977 \\
\text { Sept. } 7,1977\end{array}$ & $\begin{array}{l}25.8 \\
19.1 \\
20.6 \\
19.7\end{array}$ & 14 & 8.8 & c \\
\hline \$3 & Sugar River & $\begin{array}{l}\text { NE } 1 / 4 \text { SW } 1 / 4 \text { sec. } 34, \text { T. } 5 \text { N., R. } 8 \text { E., } \\
\text { Dane County, at bridge on State } \\
\text { Highway } 69 \text {, at Belleville, Wis. }\end{array}$ & 172 & $\begin{array}{l}\text { June } 1,1972 \\
\text { July } 31,1972 \\
\text { Nov. } 18,1973 \\
\text { Oct. } 6,1975 \\
\text { July } 26,1976\end{array}$ & $\begin{array}{c}82.7 \\
67.3 \\
102 \\
76.8 \\
61.0\end{array}$ & 45 & 33 & c \\
\hline S6 & Sugar River & $\begin{array}{l}\text { SEY/4SW1/4 sec. } 26, \text { T. } 2 \text { N., R. } 9 \text { E., } \\
\text { Green County, at town road, } 1.3 \mathrm{mi} \\
\text { southwest of Brodhead, Wis. }\end{array}$ & 523 & & & 130 & 94 & $\mathbf{a}$ \\
\hline 57 & $\begin{array}{l}\text { Badger Mill } \\
\text { Creek }\end{array}$ & $\begin{array}{l}\text { NW } / 4 \text { SE } 1 / 4 \text { sec. } 14, \text { T. } 6 \text { N., R. } 8 \text { E., } \\
\text { Dane County, at bridge on County } \\
\text { Trunk PB, } 1.0 \text { mi east of Verona, } \\
\text { Wis. }\end{array}$ & 14.9 & $\begin{array}{l}\text { June } 1,1972 \\
\text { July } 3,1972 \\
\text { Nov. } 9,1973 \\
\text { Oct. } 6,1975 \\
\text { July } 26,1976\end{array}$ & $\begin{array}{l}.13 \\
.08 \\
.33 \\
.40 \\
.02\end{array}$ & .01 & $<.01$ & c \\
\hline
\end{tabular}


Table 24. Sugar River basin station locations and low-flow characteristics-Continued.

\begin{tabular}{|c|c|c|c|c|c|c|c|c|}
\hline $\begin{array}{l}\text { Station } \\
\text { number }\end{array}$ & 1 Stream & Station location & $\begin{array}{l}\text { Drainage } \\
\text { area } \\
\left(\mathrm{mi}^{2}\right)\end{array}$ & Date & $\begin{array}{c}\text { Discharge } \\
\left(\mathrm{ft}^{3} / \mathrm{s}\right)\end{array}$ & $\begin{array}{l}\mathrm{Q}_{7,2} \mathrm{Q}_{7,1} \\
\left(\mathrm{ft}^{3} / \mathrm{s}\right)\left(\mathrm{ft}^{3}\right)\end{array}$ & 10 & racy \\
\hline 58 & $\begin{array}{l}\text { Badger Mill } \\
\text { Creek }\end{array}$ & $\begin{array}{l}\text { SEY } 1 / 4 \text { NW1/4 sec. } 22 \text {, T. } 6 \text { N., R. } 8 \text { E., } \\
\text { Dane County, at bridge on County } \\
\text { Trunk M, at Verona, Wis. }\end{array}$ & 18.9 & $\begin{array}{l}\text { July } 26,1976 \\
\text { Aug. } 3,1977 \\
\text { Sept. } 7,1977 \\
\text { Sept. } 5,1978\end{array}$ & $\begin{array}{l}2.37 \\
1.16 \\
1.02 \\
2.27\end{array}$ & 0.49 & 0.17 & c \\
\hline s9 & $\begin{array}{l}\text { Badger Mill } \\
\text { Creek }\end{array}$ & $\begin{array}{l}\text { NW } 1 / 4 \text { SW } 1 / 4 \text { sec. } 22, \text { T. } 6 \text { N., R. } 8 \text { E., } \\
\text { Dane County, at town road, } 1.0 \mathrm{mi} \\
\text { south of Verona, Wis. }\end{array}$ & 20.3 & $\begin{array}{lc}\text { June } & 1,1972 \\
\text { July } & 3,1972 \\
\text { Nov. } & 9,1973 \\
\text { Oct. } & 6,1975 \\
\text { July } & 26,1976 \\
\text { Aug. } & 3,1977 \\
\text { Sept. } & 7,1977\end{array}$ & $\begin{array}{l}2.43 \\
1.28 \\
5.86 \\
5.12 \\
2.99 \\
1.10 \\
1.31\end{array}$ & .49 & .17 & c \\
\hline $\mathbf{S 1 0}$ & $\begin{array}{l}\text { West Branch } \\
\text { Sugar River }\end{array}$ & $\begin{array}{l}\text { NE } 1 / 4 \text { SE } 1 / 4 \text { sec. } 14, \text { T. } 6 \text { N., R. } 6 \text { E., } \\
\text { Dane County, at bridge on town } \\
\text { road, } 1.1 \text { mi south of Mt. Horeb, } \\
\text { Wis. }\end{array}$ & 1.09 & $\begin{array}{l}\text { June } 2,1972 \\
\text { July } 31,1972 \\
\text { Nov. } 9,1973 \\
\text { Sept. 18, } 1974 \\
\text { Oct. 9, } 1975 \\
\text { July } 27,1976\end{array}$ & $\begin{array}{l}.44 \\
.62 \\
.41 \\
.65 \\
.30 \\
.37\end{array}$ & 5 & $\underline{5}$ & \\
\hline $\mathbf{S 1 1}$ & Allen Creek & $\begin{array}{l}\text { NE } 1 / 4 \text { NE } 1 / 4 \text { sec. } 12, T .4 \text { N., R. } 9 \text { E., } \\
\text { Green County, at bridge on County } \\
\text { Trunk T, } 0.8 \text { mi south of } \\
\text { Brooklyn, Wis. }\end{array}$ & 10.8 & $\begin{array}{l}\text { June } 1,1972 \\
\text { Aug. } 1,1972 \\
\text { Nov. } 8,1973 \\
\text { Oct. } 7,1975\end{array}$ & $\begin{array}{l}.81 \\
2.01 \\
3.23 \\
1.81\end{array}$ & .52 & .26 & c \\
\hline S13 & Allen Creek & $\begin{array}{l}\text { SE } 1 / 4 \text { SE } 1 / 4 \text { sec. } 27, T .4 \text { N., R. } 10 \text { E., } \\
\text { Rock County, at sewage-treatment } \\
\text { plant, at Evansville, Wis. }\end{array}$ & 27.5 & $\begin{array}{l}\text { June } 1,1972 \\
\text { Aug. } 1,1972 \\
\text { Nov. } 8,1973 \\
\text { Oct. } 7,1975 \\
\text { July } 26,1976\end{array}$ & $\begin{array}{c}6.49 \\
6.56 \\
12.8 \\
6.75 \\
2.95\end{array}$ & 2.2 & 1.1 & c \\
\hline$S 14$ & $\begin{array}{l}\text { Little Sugar } \\
\text { River }\end{array}$ & $\begin{array}{l}\text { SW } 1 / 4 \text { SE } 1 / 4 \text { sec. } 14, \text { T. } 4 \text { N., R. } 7 \text { E., } \\
\text { Green County, et bridge on town } \\
\text { road, } 300 \text { ft east of junction of } \\
\text { State Highway } 39 \text { and State Highway } \\
69 \text {, at New Glarus, Wis. }\end{array}$ & 22.4 & $\begin{array}{l}\text { June } 1,1972 \\
\text { Aug. } 1,1972 \\
\text { Nov. } 8,1973 \\
\text { Oct. } 7,1975 \\
\text { Julv } 27,1976\end{array}$ & $\begin{array}{l}12.8 \\
13.3 \\
14.2 \\
9.44 \\
7.29\end{array}$ & 5.4 & 3.6 & c \\
\hline$S 16$ & $\begin{array}{l}\text { West Branch } \\
\text { Little Sugar }\end{array}$ & $\begin{array}{l}\text { SW1/4SW1/4 sec. } 8, \text { T. } 3 \text { N., R. } 8 \text { E., } \\
\text { Green County, at bridge on town } \\
\text { road, about } 400 \mathrm{ft} \text { northwest of } \\
\text { sewage-treatment plant, at } \\
\text { Monticello, Wis. }\end{array}$ & 33.6 & $\begin{array}{l}\text { June } 1,1972 \\
\text { Aug. } 1,1972 \\
\text { Nov. } 8,1973 \\
\text { Oct. } 6,1975 \\
\text { Julv } 27,1976\end{array}$ & $\begin{array}{l}18.8 \\
17.7 \\
20.3 \\
14.4 \\
12.4\end{array}$ & 9.2 & 6.4 & c \\
\hline$S 18$ & Juda Branch & $\begin{array}{l}\text { NE1/4NW1/4 sec. 1, T. } 1 \text { N., R. } 8 \text { E., } \\
\text { Green County, at bridge on State } \\
\text { Highways } 11 \text { and } 81 \text {, at Juda, Wis. }\end{array}$ & 8.13 & $\begin{array}{l}\text { Aug. } 27,1968 \\
\text { Oct. 18, } 1968 \\
\text { June } 1,1972 \\
\text { Aug. } 1,1972 \\
\text { Nov. } 8,1973 \\
\text { Oct. } 10,1975 \\
\text { Julv } 27,1976\end{array}$ & $\begin{array}{l}1.74 \\
2.03 \\
3.32 \\
2.80 \\
5.19 \\
3.06 \\
2.40\end{array}$ & .80 & .39 & c \\
\hline $\mathbf{S 2 2}$ & Swan Creek & $\begin{array}{l}\text { SW } 1 / 4 \text { SW } 1 / 4 \text { sec. } 13, \text { T. } 2 \text { N., R. } 10 \text { E., } \\
\text { Rock County, at sewage-treatment } \\
\text { plant, at Orfordville, Wis. }\end{array}$ & 1.00 & $\begin{array}{l}\text { June } 1,1972 \\
\text { Aug. } 1,1972 \\
\text { Nov. } 8,1973 \\
\text { Oct. } 7,1975 \\
\text { Julv } 26,1976\end{array}$ & $\begin{array}{l}40 \\
40 \\
40 \\
40 \\
2_{0}^{0}\end{array}$ & 0 & 0 & c \\
\hline
\end{tabular}

${ }^{1}$ Based on 1971 report by Wisconsin Department of Natural Resources.

2 Streambed is dry.

${ }^{4}$ Negligible discharge--water pondad, unable to measure velocity.

${ }^{5}$ No estimate possible--discharge is primarily effluent. 


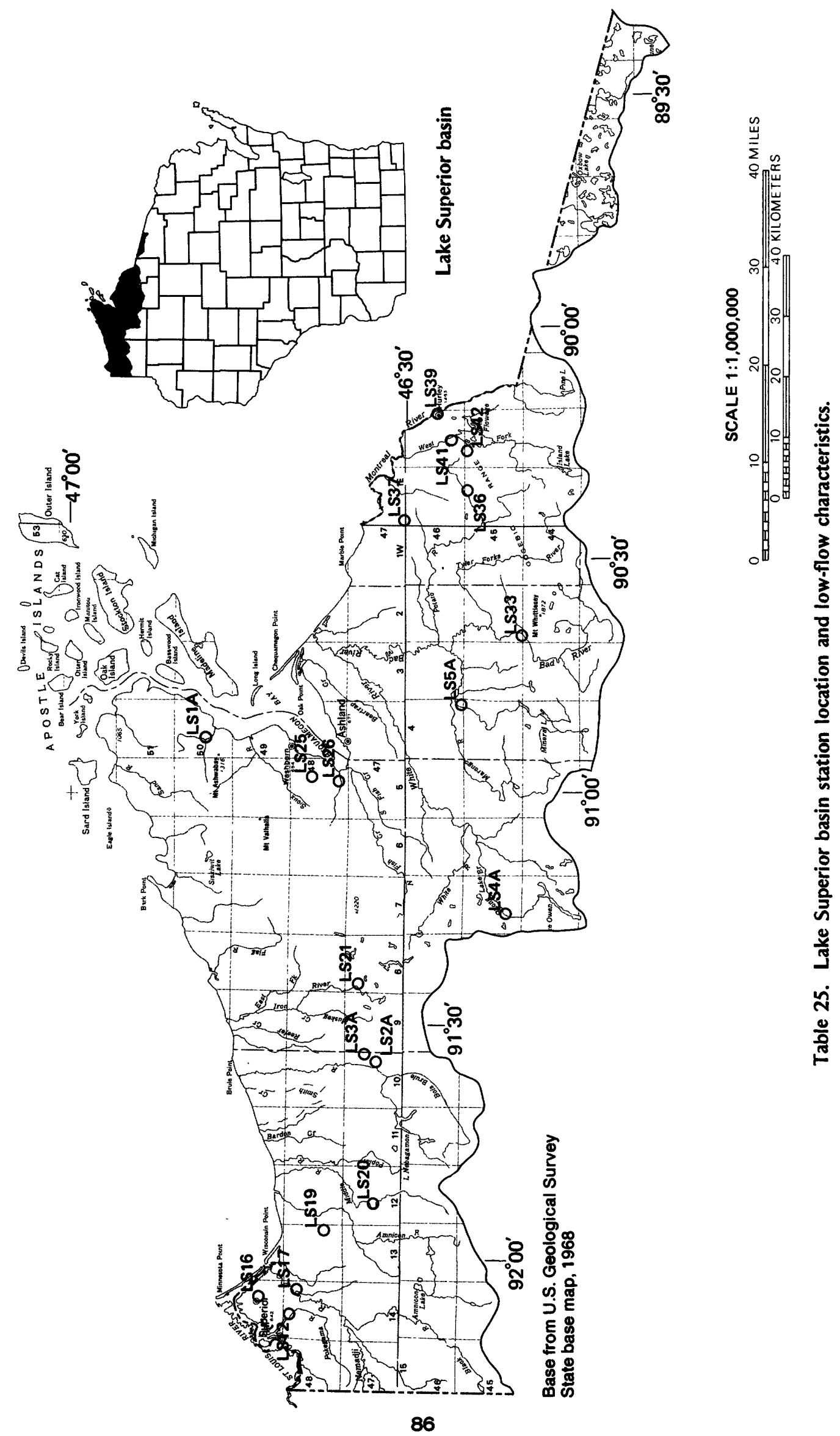


Table 25. Lake Superior basin station location and low-flow characteristics-Continued.

\begin{tabular}{|c|c|c|c|c|c|c|}
\hline $\begin{array}{l}\text { Station } \\
\text { number }\end{array}$ & $\underset{\text { name }}{\text { Stream }}$ & Station location & $\begin{array}{c}\text { Drainage } \\
\text { area } \\
\left(\mathrm{mi}^{2}\right)\end{array}$ & Date & $\begin{array}{c}\text { Discharge } \\
\left(\mathrm{ft}^{3} / \mathrm{s}\right)\end{array}$ & 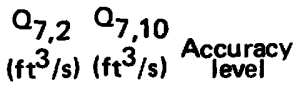 \\
\hline
\end{tabular}

LS12 Pokegama River

Pokegama River

LS16 Lake Superior tributary

LS17 Crawford Creek

LS19 Bear Creek

LS20 Middle River

LS21 Iron River

LS25 Boyd Creek

LS26 Whittlesey Creek tributary

LS33 Bad River

LS36 Alder Creek tributary
NE $1 / 4$ SW $1 / 4$ sec. 10, T. 48 N., R. 14 W., Douglas County, at sewage-treatment plant, at Superior Village, Wis.

NW $1 / 4$ NW $1 / 4$ sec. 10, T. 48 N., R. 14 W. Douglas County, at bridge on town road, 0.25 mi south of State Highway $105,0.75 \mathrm{mi}$ west of State Highway 35, in South Superior, Wis.

SW1/4SE $1 / 4$ sec. 25, T. 49 N., R. 14 W. Douglas County, at culvert on Stinson Avenue, $2.5 \mathrm{mi}$ southeast of post office, at Superior, Wis.

NE $1 / 1 / N^{1} 1 / 4$ sec. $14, T .48$ N., R. 13 W., Douglas County, at bridge on town road, 1.85 mi southeast of South Superior, Wis.

SW $1 / 4$ SW $1 / 4$ sec. 9, T. 48 N., R. 13 W. Douglas County, at bridge on County Trunk Z, $300 \mathrm{ft}$ upstream from Douglas County Hospital and Sanatorium sewage-treatmént plant, $4.3 \mathrm{mi}$ southeast of South Superior, Wis.

SE $1 / 4 N W 1 / 4$ sec. $21, T .47$ N., R. 12 W., Douglas County, at Middle River Sanatorium sewage-treatment plant, $1.4 \mathrm{mi}$ east of Hines, Wis.

NW $1 / 4 N^{1} / 1 / 4$ sec. 7, T. 47 N., R. 8 W., Bayfield County, at country bridge and sewage-treatment plant, at Iron River, Wis.

NE $1 / 4 S^{1} 1 / 4$ sec. 22, T. 48 N., R. 5 W., Bayfield County, at bridge on town road, $4.5 \mathrm{mi}$ nor thwest of courthouse, at Ashland, Wis.

SW $1 / 4 N W 1 / 4$ sec. 35, T. 48 N., R. 5 W., Bayfield County, just below confluence of two tributaries, at country road $0.3 \mathrm{mi}$ south of Ondassagon School, $4.0 \mathrm{mi}$ east of Ashland, Wis.

NW1/4NE $1 / 4$ sec. 6, T. 44 N., R. 2 W., Ashland County, at State Highway 13 at Mellen Wis.

NW1/2NW1/4 sec. 2, T. 45 N., R. 1 E., Iron County, on road past sewage lagoon, $1.0 \mathrm{mi}$ northwest of Iron Belt, Wis.

25.8

26.3 Oct. 17,1972

May 23, 1973

Sept. 18, 1973

Aug. 1, 1973

July 24,1975

Aug. 21, 1975

Aug. 3, 1976

.50

July 25, 1975

Aug. 20, 1975

Aug. 3, 1976

Aug. 24, 1977

7.97 Aug. 26, 1970

July 25, 1975

Aug. 21, 1975

Aug. 3, 1976

Aug. 24, 1977

3.56 Oct. 17, 1972

May 23, 1973

Sept. 18, 1973

Aug. 1, 1974

July 24, 1975

Aug. 21, 1975

31.6 Oct. 17, 1972

May 23, 1973

Sept. 18, 1973

Aug. 1, 1974

July 24, 1975

Aug. 21, 1975

19.1 Oct. 17, 1972

May 23, 1973

Sept. 18, 1973

July 31, 1974

July 23, 1975

Aug. 20, 1975

3.11 July 22, 1975

Aug. 20, 1975

Aug. 4, 1976

Aug. 25, 1977

.94

Oct. 18, 1972

May 22, 1973

Sept. 18, 1973

July 31,1974

July 22, 1975

Aug. 20,1975

Aug. 4, 1976

Aug. 25, 1977

105

Aug. 15, 1972

Oct. 19, 1972

May 22, 1973

Sept. 19, 1973

June 26, 1974

July 30,1974

July 22, 1975

Aug. 19, 1975

1.24 Aug. 15, 1972

Oct. 18,1972

May 21, 1973

Sept. 19, 1973

July 21, 1975

Aug. 19, 1975

\subsection{6 \\ 3.40 \\ $4^{4.33}$ \\ $<.01$}

0.020

c

5.88

5.88

4.38

20

$<.01$

$4^{4} .04$
$4_{0}^{0}$

.09

4.04

${ }_{2}^{2}{ }_{2}^{0}$

12.6

20.9

5.09

5.24

4.39

1.07

16.8

19.3

10.8

13.8

13.5

12.2

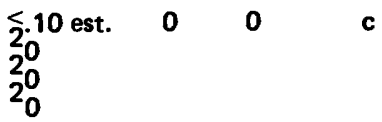

.51

.74

.56

.74

.64

.65

.67

.59

$3_{59.6}$

77.4

96.1

16.2

51.7

16.2

11.7

6.56

.96

.79

1.78

.86

.42
36

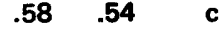

12

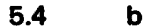

${ }^{8} .40 \quad{ }^{8} .36$ 
Table 25. Lake Superior basin station location and low-flow characteristics-Continued.

\begin{tabular}{|c|c|c|c|c|c|c|c|c|}
\hline $\begin{array}{l}\text { Station } \\
\text { number }\end{array}$ & 1 name & Station location & $\begin{array}{l}\text { Drainage } \\
\text { area } \\
\left(\mathrm{mi}^{2}\right)\end{array}$ & Date & $\begin{array}{c}\text { Discharge } \\
\left(\mathrm{ft}^{3} / \mathrm{s}\right)\end{array}$ & $\begin{array}{l}\mathrm{a}_{7,2} \mathrm{a}_{7} \\
\left(\mathrm{ft}^{3} / \mathrm{s}\right)\left(\mathrm{ft}^{3}\right.\end{array}$ & & $\begin{array}{c}\text { Accuracy } \\
\text { level }\end{array}$ \\
\hline LS37 & Vaughn Creek & $\begin{array}{l}\text { SE } 1 / 4 N W 1 / 4 \text { sec. } 6, \text { T. } 46 \text { N., R. } 1 \text { E., } \\
\text { Iron County, at bridge on State } \\
\text { Highway } 122 \text {, at Saxon, Wis. }\end{array}$ & 1.11 & $\begin{array}{l}\text { Aug. } 15,1972 \\
\text { Oct. } 19,1972 \\
\text { May } 22,1973 \\
\text { Sept. } 19,1973 \\
\text { July } 30,1974 \\
\text { July } 22,1975 \\
\text { Aug. } 19,1975\end{array}$ & $\begin{array}{l}{ }^{4}{ }_{0}^{0} \\
4_{0}^{.16} \\
4_{0}^{0} \\
4_{0}^{0}\end{array}$ & 0 & 0 & c \\
\hline LS39 & Montreal River & $\begin{array}{l}\text { NW1/4SE1/4 sec. } 24, \text { T. } 46 \text { N., R. } 2 \text { E., } \\
\text { Iron County, at Aurora Street } \\
\text { bridge between Hurley, Wis., and } \\
\text { Ironwood, Mich. }\end{array}$ & 63.0 & & & 4.7 & 2.4 & $\mathbf{a}$ \\
\hline LS41 & $\begin{array}{l}\text { West Fork } \\
\text { Montreal River }\end{array}$ & $\begin{array}{l}\text { NE } 1 / 4 \text { SW } 1 / 4 \text { sec. } 27, \text { T. } 46 \text { N., R. } 2 \text { E., } \\
\text { Iron County, at sewage-treatment } \\
\text { plant, } 0.1 \text { mi downstream from } \\
\text { State Highway } 77 \text { bridge, at } \\
\text { Montreal, Wis. }\end{array}$ & 78.5 & $\begin{array}{l}\text { Aug. } 14,1972 \\
\text { Oct. } 19,1972 \\
\text { May } 21,1973 \\
\text { Sept. } 19,1973 \\
\text { July } 30,1974 \\
\text { July } 21,1975 \\
\text { Aug. } 19,1975\end{array}$ & $\begin{array}{l}84.1 \\
92.4 \\
93.5 \\
138 \\
170 \\
182 \\
159\end{array}$ & 6 & 6 & \\
\hline \multirow[t]{2}{*}{ LS42 } & $\begin{array}{l}\text { West Fork } \\
\text { Montreal River } \\
\text { tributary }\end{array}$ & $\begin{array}{l}\text { NE } 1 / 4 \text { NW1/4 sec. } 5, \text { T. } 45 \text { N., R. } 2 \text { E., } \\
\text { Iron County, at sewage-treatment } \\
\text { plant, at Pence, Wis. }\end{array}$ & .14 & & & & & \\
\hline & $\begin{array}{l}\text { West Fork } \\
\text { Montreal River }\end{array}$ & $\begin{array}{l}\text { SE } 1 / 4 \text { NE } 1 / 4 \text { sec. } 5, \text { T. } 45 \text { N., R. } 2 \text { E., } \\
\text { Iron County, at country road, } \\
0.75 \text { mi southeast of Pence, Wis. }\end{array}$ & .53 & $\begin{array}{l}\text { Oct. } 19,1973 \\
\text { May } 21,1973 \\
\text { Aug. } 14,1972 \\
\text { Sept. 19, } 1973 \\
\text { July } 30,1974 \\
\text { July } 21,1975 \\
\text { Aug. } 19,1975\end{array}$ & $\begin{array}{l}20 \\
2.32 \\
20 \\
20 \\
.33 \\
.03 \\
<.01\end{array}$ & 0 & 0 & c \\
\hline LS1A & Pikes Creek & $\begin{array}{l}\text { NW1/4NE } 1 / 4 \text { sec. } 28, \text { T. } 50 \text { N., R. } 4 \text { W., } \\
\text { Beyfield County, at DNR fish } \\
\text { hatchery, } 3.0 \text { mi southwest of. } \\
\text { Bayfield, Wis. }\end{array}$ & 30.4 & $\begin{array}{l}\text { July } 23,1975 \\
\text { Aug. } 3,1976 \\
\text { Aug. } 25,1977 \\
\text { Nov. } 15,1978\end{array}$ & $\begin{array}{l}8.05 \\
7.75 \\
6.81 \\
13.1\end{array}$ & 8.0 & 6.2 & c \\
\hline LS2A & $\begin{array}{l}\text { Little Bois } \\
\text { Brule River }\end{array}$ & $\begin{array}{l}\text { NW1/4NW1/4 sec. } 35, T .47 \text { N., R. } 10 \text { W., } \\
\text { Douglas County, at bridge on State } \\
\text { Highway } 27,2.5 \text { mi southwest of } \\
\text { Brule, Wis. }\end{array}$ & & $\begin{array}{l}\text { Aug. } 3,1976 \\
\text { Aug. } 24,1977 \\
\text { Nov. } 16,1978\end{array}$ & $\begin{array}{l}4.32 \\
2.27 \\
2.86\end{array}$ & 2.8 & 2.5 & c \\
\hline LS3A & Sandy Run & $\begin{array}{l}\text { SW1 } 1 / 4 N^{1} / 4 \text { sec. } 13, T .47 \text { N., R. } 10 \text { W., } \\
\text { Douglas County, at culvert on U.S. } \\
\text { Highway } 2,0.8 \text { mi east of Brule, Wis. }\end{array}$ & 3.04 & $\begin{array}{l}\text { July } 23,1975 \\
\text { Aug. } 20,1975 \\
\text { Aug. } 3,1976 \\
\text { Aug. } 24,1977\end{array}$ & $\begin{array}{l}4.08 \\
4.28 \\
3.66 \\
3.52\end{array}$ & 3.4 & 3.2 & c \\
\hline LS4A & $\begin{array}{l}\text { Long Lake } \\
\text { Branch }\end{array}$ & $\begin{array}{l}\text { NE } 1 / 4 S^{1} 1 / 4 \text { sec. } 29, \text { T. } 45 \text { N., R. } 7 \text { W., } \\
\text { Bayfield County, at trail crossing } \\
\text { just downstream from Drummond Lake, } \\
0.8 \text { mi north of Drummond, Wis. }\end{array}$ & 24.5 & $\begin{array}{l}\text { Feb. } 12,1975 \\
\text { Mar. } 19,1975 \\
\text { July } 23,1975 \\
\text { Aug. } 19,1975 \\
\text { Aug. } 4,1976 \\
\text { June } 10,1977 \\
\text { Aug. } 25,1977\end{array}$ & $\begin{array}{l}5.74 \\
5.41 \\
4.45 \\
.48 \\
.41 \\
2_{0}^{0}\end{array}$ & $\underline{6}$ & $\underline{6}$ & \\
\hline LS5A & $\begin{array}{l}\text { Brunsweiler } \\
\text { River }\end{array}$ & $\begin{array}{l}\text { NW } 1 / 4 N E 1 / 4 \text { sec. } 1, T .45 \text { N., R. } 4 \text { W., } \\
\text { Ashland County, at bridge on State } \\
\text { Highway } 13,0.8 \text { mi southeast of } \\
\text { Marengo, Wis. }\end{array}$ & 62.9 & $\begin{array}{l}\text { Aug. } 26,1970 \\
\text { Aug. 19, } 1975 \\
\text { Aug. } 4,1976 \\
\text { Aug. } 24,1977\end{array}$ & $\begin{array}{c}6.09 \\
6.48 \\
6.70 \\
11.4\end{array}$ & 10 & 6.4 & c \\
\hline
\end{tabular}

\footnotetext{
'Based on 1966 report by Wisconsin Department of Natural Resources (DNR). Site lettered "A" was not included in 1966 DNR report.

${ }^{2}$ Streambed is dry.

3 Additional discharge measurements are available.

${ }^{4}$ Negligible discharge--water ponded, unable to measure velocity.

5 Discharge is primarily effluent.

6 No estimate possible due to regulation upstream.

8 Low-flow characteristics and discharge measured include about $0.30 \mathrm{ft}^{3} / \mathrm{s}$ from sewage lagoon.
} 
Table 26. Trempealeau-Buffalo River basin station locations and low-flow characteristics.

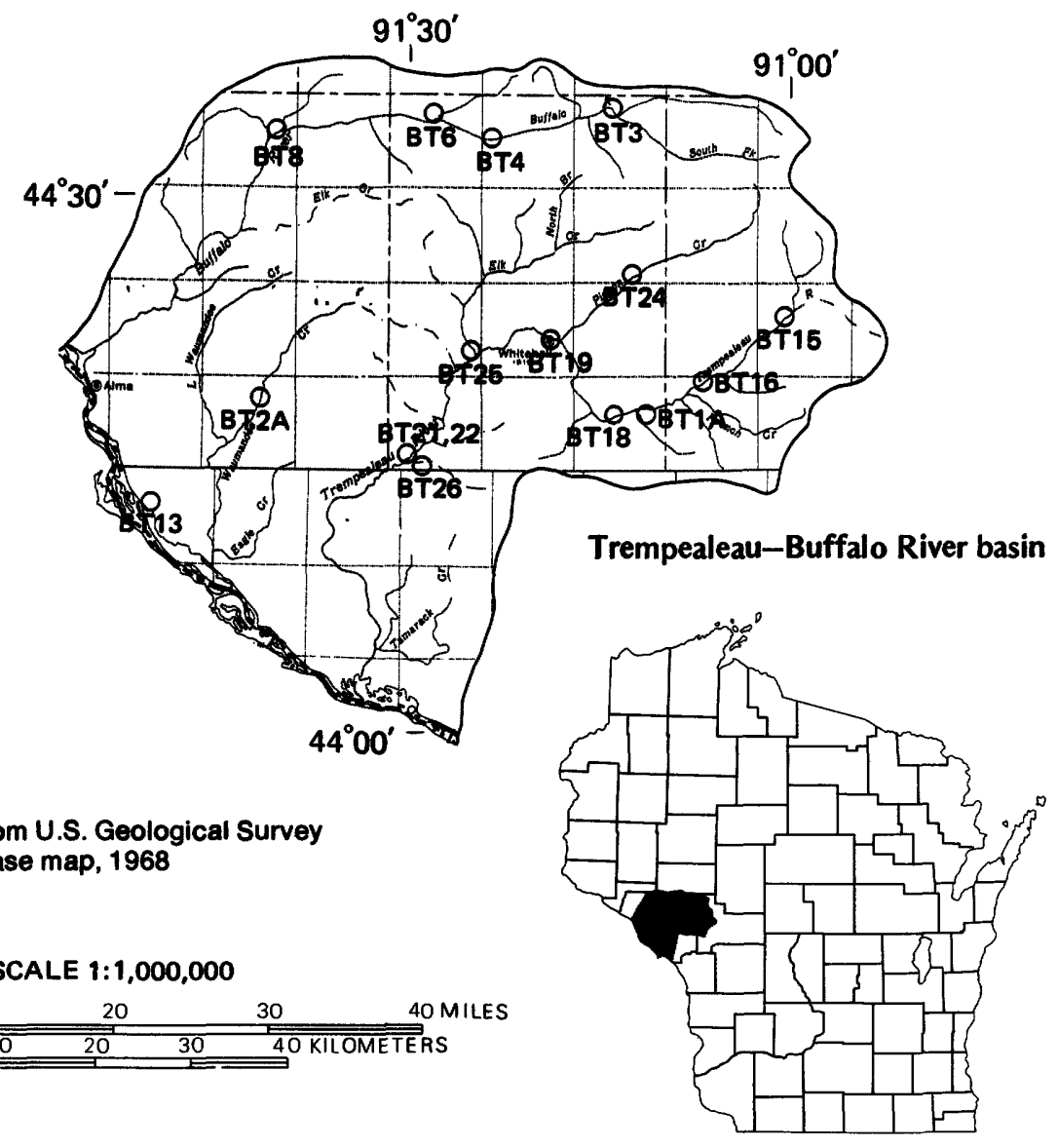

Base from U.S. Geological Survey

State base map, 1968

SCALE 1:1,000,000

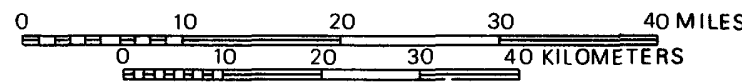

\begin{tabular}{|c|c|c|c|c|c|c|c|c|}
\hline $\begin{array}{l}\text { Station } \\
\text { number }\end{array}$ & Stream & Station location & $\begin{array}{l}\text { Drainage } \\
\text { area } \\
\left(\mathrm{mi}^{2}\right)\end{array}$ & Date & $\begin{array}{c}\text { Discharge } \\
\left(\mathrm{ft}^{3} / \mathrm{s}\right)\end{array}$ & $\begin{array}{l}Q_{7,2} Q_{7} \\
\left(f^{3} / s\right)(f t\end{array}$ & $\begin{array}{l}7,10 \\
3 / 51\end{array}$ & $\begin{array}{c}\text { Accuracy } \\
\text { level }\end{array}$ \\
\hline BT3 & Buffalo River & $\begin{array}{l}\text { SW1/NWW/ sec. 9, T. } 24 \text { N., R. } 7 \text { W., } \\
\text { Trempealeau County, at bridge on } \\
\text { County Trunk O, } 1.3 \text { mi west of } \\
\text { Osseo, Wis. }\end{array}$ & 73.7 & $\begin{array}{l}\text { Aug. } 12,1970 \\
\text { Aug. } 9,1972 \\
\text { June } 25,1973 \\
\text { Sept. } 12,1973 \\
\text { Aug. } 21,1976 \\
\text { Sept. } 15,1977\end{array}$ & $\begin{array}{l}24.7 \\
50.3 \\
68.8 \\
40.0 \\
19.7 \\
17.1\end{array}$ & 16 & 11 & c \\
\hline BT4 & Buffalo River & $\begin{array}{l}\text { SW\%SEY// sec. 18, T. } 24 \text { N., R. } 8 \text { W., } \\
\text { Trempealeau County, at footbridge } \\
\text { in city park, at Strum, Wis. }\end{array}$ & 124 & $\begin{array}{l}\text { Aug. } 9,1972 \\
\text { June } 26,1973 \\
\text { Sept. } 12,1973 \\
\text { Aug. } 21,1976 \\
\text { Sept. 15, } 1977\end{array}$ & $\begin{array}{c}77.9 \\
139 \\
80.5 \\
38.0 \\
35.1\end{array}$ & 32 & 20 & c \\
\hline BT6 & Buffalo River & $\begin{array}{l}\text { NE } 1 / 4 \text { SE } \% \text { sec. } 9, \text { T. } 24 \text { N., R. } 9 \text { W., } \\
\text { Trempealeau County, at bridge on } \\
\text { State Highway } 93 \text {, at Eleva, Wis. }\end{array}$ & 165 & $\begin{array}{l}\text { Aug. } 9,1972 \\
\text { June } 26,1973 \\
\text { Sept. } 13,1973 \\
\text { Aug. } 21,1976 \\
\text { Sept. 15, } 1977\end{array}$ & $\begin{array}{l}96.4 \\
204 \\
112 \\
54.7 \\
44.8\end{array}$ & 38 & 23 & c \\
\hline BT8 & Buffalo River & $\begin{array}{l}\text { NW/4SEY// sec. 14, T. } 24 \text { N., R. } 11 \text { W., } \\
\text { Buffalo County, at sewage-treatment } \\
\text { plant, } 0.5 \text { mi downstream from } \\
\text { County Trunk H bridge, at Mondovi, } \\
\text { Wis. }\end{array}$ & 238 & $\begin{array}{l}\text { Aug. } 9,1972 \\
\text { June } 26,1973 \\
\text { Sept. 12, } 1973 \\
\text { Aug. } 21,1976 \\
\text { Sept. 15, } 1977\end{array}$ & $\begin{array}{l}138 \\
278 \\
164 \\
85.7 \\
85.7\end{array}$ & 64 & 41 & c \\
\hline
\end{tabular}


Table 26. Trempealeau-Buffalo River basin station locations and low-flow characteristics-Continued.

\begin{tabular}{|c|c|c|c|c|c|c|c|c|}
\hline $\begin{array}{l}\text { Station } \\
\text { number }\end{array}$ & Stream & Station location & $\begin{array}{l}\text { Drainage } \\
\text { area } \\
\left(\mathrm{mi}^{2}\right)\end{array}$ & Date & $\begin{array}{l}\text { Discharge } \\
\left(\mathrm{ft}^{3} / \mathrm{s}\right)\end{array}$ & $\begin{array}{l}\mathrm{O}_{7,2} \mathrm{O}_{7} \\
\left(\mathrm{ft}^{3} / \mathrm{s}\right)\left(\mathrm{ft}^{3}\right.\end{array}$ & $3 / 5) \mathrm{AC}$ & $\begin{array}{c}\text { Accuracy } \\
\text { level }\end{array}$ \\
\hline \multirow[t]{2}{*}{ BT13 } & Cochrane Ditch & $\begin{array}{l}\text { SW } 1 / 4 N^{1} 1 / 4 \text { sec. } 8, T .20 \text { N., R. } 12 \mathrm{~W} . \text {, } \\
\text { Buffalo County, at sewage-treatment } \\
\text { plant near southwest corporate limits } \\
\text { of Cochrane, Wis. }\end{array}$ & 8.66 & & & & & \\
\hline & Cochrane Ditch & $\begin{array}{l}\mathrm{SE} 1 / 4 \mathrm{SE}^{1} / 4 \text { sec. } 8, \mathrm{~T} .20 \mathrm{~N} ., \mathrm{R} .12 \mathrm{~W} . \text {, } \\
\text { Buffalo County, at country road } \\
\text { bridge, } 0.5 \mathrm{mi} \text { south of Cochrane, } \\
\text { Wis. }\end{array}$ & 8.87 & $\begin{array}{l}\text { Aug. } 9,1972 \\
\text { June } 26,1973 \\
\text { Sept. } 12,1973 \\
\text { Aug. } 9,1977 \\
\text { Sept. } 14,1977\end{array}$ & $\begin{array}{l}9.59 \\
8.72 \\
7.58 \\
4.07 \\
5.45\end{array}$ & 4.4 & 3.5 & c \\
\hline BT15 & $\begin{array}{l}\text { Trempealeau } \\
\text { River }\end{array}$ & $\begin{array}{l}\text { NW1/4SE1/4 sec. } 17, \text { T. } 22 \text { N., R. } 5 \text { W., } \\
\text { Jackson County, at State Highway } \\
95 \text { bridge, at Hixton, Wis. }\end{array}$ & 58.3 & $\begin{array}{l}\text { Aug. } 10,1972 \\
\text { June } 26,1973 \\
\text { Sept. 12, } 1973 \\
\text { Aug. } 21,1976 \\
\text { Sept. 15, } 1977\end{array}$ & $\begin{array}{l}26.8 \\
54.2 \\
39.5 \\
23.3 \\
22.9\end{array}$ & 19 & 14 & c \\
\hline BT16 & $\begin{array}{l}\text { Trempealeau } \\
\text { River }\end{array}$ & $\begin{array}{l}\text { NE } 1 / 4 S^{1} 1 / 4 \text { sec. } 5, \text { T. } 21 \text { N., R. } 6 \text { W., } \\
\text { Jackson County, at County Trunk P, } \\
0.4 \text { mi north of Taylor, Wis. }\end{array}$ & 110 & $\begin{array}{l}\text { Aug. } 10,1972 \\
\text { June } 27,1973 \\
\text { Sept. 12, 1973 } \\
\text { Aug. } 20,1976 \\
\text { Sept. 14, } 1977\end{array}$ & $\begin{array}{c}3_{52.8} \\
124 \\
74.5 \\
43.8 \\
38.5\end{array}$ & 35 & 25 & b \\
\hline BT18 & $\begin{array}{l}\text { Trempealeau } \\
\text { River }\end{array}$ & $\begin{array}{l}\text { NW1/4SE1/4 sec. } 16, T .21 \text { N., R. } 7 \text { W., } \\
\text { Trempealeau County, at sewage- } \\
\text { treatment plant, at Blair, Wis. }\end{array}$ & 176 & $\begin{array}{l}\text { Aug. } 10,1972 \\
\text { Sept. 12,1973 } \\
\text { Jan. } 9,1974 \\
\text { Aug. 20, } 1976 \\
\text { Sept. 14, } 1977\end{array}$ & $\begin{array}{c}84.1 \\
108 \\
85.0 \\
62.9 \\
51.8\end{array}$ & 47 & 34 & c \\
\hline BT19 & $\begin{array}{l}\text { Trempealeau } \\
\text { River }\end{array}$ & $\begin{array}{l}\text { SE } 1 / 4 N^{N} 1 / 4 \text { sec. } 23, \text { T. } 22 \text { N., R. } 8 \text { W., } \\
\text { Trempealeau County, at U.S. Highway } \\
53 \text { bridge, at Whitehall, Wis. }\end{array}$ & 317 & $\begin{array}{l}\text { Aug. } 10,1972 \\
\text { June } 27,1972 \\
\text { Sept. 12, } 1973 \\
\text { Aug. } 20,1976 \\
\text { Sept. 14, } 1977\end{array}$ & $\begin{array}{l}159 \\
391 \\
243 \\
125 \\
105\end{array}$ & 93 & 63 & c \\
\hline $\begin{array}{l}\text { BT21, } \\
22\end{array}$ & $\begin{array}{l}\text { Trempealeau } \\
\text { River }\end{array}$ & $\begin{array}{l}\text { NW1/4SW1/4 sec. } 32, \text { T. } 21 \text { N., R. } 9 \text { W., } \\
\text { Trempealeau County, at State } \\
\text { Highways } 93 \text { and } 95 \text {, in Arcadia, Wis. }\end{array}$ & 552 & & & 1651 & 118 & $\mathbf{a}$ \\
\hline \multirow[t]{2}{*}{ ВT24 } & Pigeon Creek & $\begin{array}{l}\text { SW1/4SE } 1 / 4 \text { sec. } 34, \mathrm{~T} .23 \mathrm{~N} ., \mathrm{R} .7 \mathrm{~W} . \text {, } \\
\text { Trempealeau County, } 300 \mathrm{ft} \text { downstream } \\
\text { from dam, in Pigeon Falls, Wis. }\end{array}$ & 58.4 & $\begin{array}{l}\text { Aug. } 10,1972 \\
\text { June } 27,1973 \\
\text { Sept. 12, 1973 } \\
\text { Aug. 20, } 1976 \\
\text { Sept. 14, } 1977\end{array}$ & $\begin{array}{l}31.3 \\
77.1 \\
44.1 \\
25.2 \\
21.5\end{array}$ & 20 & 14 & c \\
\hline & Pigeon Creek & $\begin{array}{l}\text { NE } 1 / 4 N W 1 / 4 \text { sec. } 3, T .22 \text { N., R. } 7 \text { W., } \\
\text { Trempealeau County, at sewage- } \\
\text { treatment plant, } 0.6 \text { mi southeast } \\
\text { of Pigeon Falls, Wis. }\end{array}$ & 60.3 & & & & & \\
\hline BT25 & Elk Creek & $\begin{array}{l}\text { SE1/4NW1/4 sec. } 25, T .22 \text { N., R. } 9 \text { W., } \\
\text { Trempealeau County, at State } \\
\text { Highways } 93 \text { and } 121 \text { bridge, in } \\
\text { Independence, Wis. }\end{array}$ & 101 & $\begin{array}{l}\text { Aug. } 10,1972 \\
\text { June } 27,1973 \\
\text { Sept. 12, } 1973 \\
\text { Aug. } 20,1976 \\
\text { Sept. 14, } 1977\end{array}$ & $\begin{array}{c}53.0 \\
116 \\
75.1 \\
39.4 \\
28.4\end{array}$ & 26 & 17 & c \\
\hline ВT26 & Meyers Valley & $\begin{array}{l}\text { NW } 1 / 4 N E 1 / 4 \text { sec. } 6, T .20 \text { N., R. } 9 \text { W., } \\
\text { Trempealeau County, at bridge on } \\
\text { country road, } 0.4 \text { mi south of } \\
\text { Arcadia, Wis. }\end{array}$ & 5.57 & $\begin{array}{l}\text { Aug. } 19,1976 \\
\text { Aug. } 8,1977 \\
\text { Sept. } 14,1977 \\
\text { Sept. } 11,1978\end{array}$ & $\begin{array}{l}1.28 \\
1.22 \\
1.28 \\
4.12\end{array}$ & 1.2 & .76 & c \\
\hline BT1A & Tappen Coulee & $\begin{array}{l}\text { SE } 1 / 4 S^{1} 1 / 4 \text { sec. } 16, T .21 \text { N., R. } 7 \text { W., } \\
\text { Trempealeau County, at bridge on } \\
\text { town road, at Blair, Wis. }\end{array}$ & 4.44 & $\begin{array}{l}\text { Aug. } 19,1976 \\
\text { Aug. } 8,1977 \\
\text { Sapt. } 14,1977\end{array}$ & $\begin{array}{l}.80 \\
.55 \\
.61\end{array}$ & .49 & .30 & c \\
\hline BT2A & Waumandee Creek & $\begin{array}{l}\text { SW1/4NW1/4 sec. } 2, \text { T. } 21 \text { N., R. } 11 \text { W., } \\
\text { Buffalo County, at bridge on private } \\
\text { road, } 1.6 \text { mi northeast of Waumandee, } \\
\text { Wis. }\end{array}$ & 40.2 & $\begin{array}{l}\text { Aug. } 20,1976 \\
\text { Aug. } 9,1977 \\
\text { Sept. } 14,1977\end{array}$ & $\begin{array}{l}18.5 \\
13.8 \\
13.6\end{array}$ & 12 & 7.8 & c \\
\hline
\end{tabular}

1 Based on 1972 report by Wisconsin Department of Natural Resources (DNR). Site lettered "A" was not included in 1972 DNR report.

${ }^{3}$ Additional discharge measurements are available. 
Table 27. Twin-Kewaunee River basin and Door County station locations and low-flow characteristics.

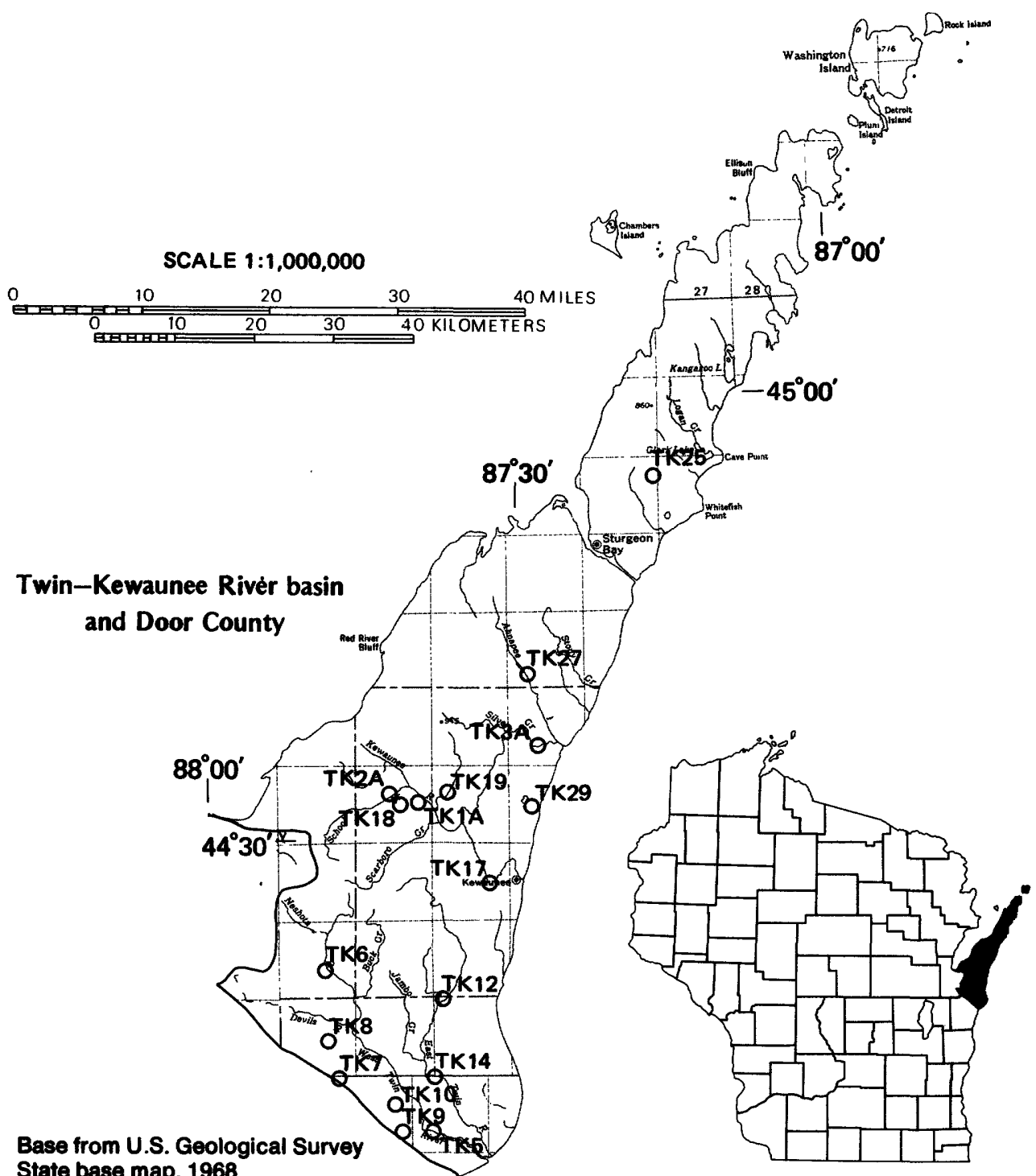

State base map, 1968

\begin{tabular}{|c|c|c|c|c|c|c|}
\hline $\begin{array}{l}\text { Station } \\
\text { number }\end{array}$ & $\underset{\text { name }}{\text { Stream }}$ & Station location & $\begin{array}{l}\text { Drainage } \\
\text { area } \\
\left(\mathrm{mi}^{2}\right)\end{array}$ & Date & $\begin{array}{c}\text { Discharge } \\
\left(\mathrm{ft}^{3} / \mathrm{s}\right)\end{array}$ & 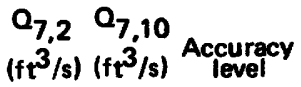 \\
\hline
\end{tabular}

TK5 West Twin River

SW $1 / 4$ NE $1 / 4$ sec. 29, T. 20 N., R. 24 E. Manitowoc County, et County

Trunk B, 2.9 mi northeast of

Rockwood, Wis.

TK6 Neshota River

Neshota River tributary
SE $1 / 4$ SE $1 / 4$ sec. 22 , T. 22 N., R. 22 E., Brown County, at bridge on River Roed, $1.4 \mathrm{mi}$ northeast of Denmark, Wis.

NE $1 / 4$ SW $1 / 4$ sec. 27, T. 22 N., R. 22 E. Brown County, at sewage-treatment plant, at Denmark, Wis.
Oct. 3, 1968

Oct. 10,1972

Aug. 2, 1973

Oct. 21, 1975

July 12, 1976

Sept. 15, 1976

34.5

Oct. 4, 1968

July 19, 1972

Oct. 11, 1972

Aug. 2, 1973

Oct. 21, 1975

Julv 13, 1976

Sept. 14, 1976

1.01
16.7

33.1

24.7

24.7

13.7

15.0

2.50

1.26

5.71

2.42

2.22

1.06

$1.0 \mathrm{~B}$
12

$9.2 \quad \mathrm{c}$

.57 c 
Table 27. Twin-Kewaunee River basin and Door County station locations and low-flow characteristics-Continued.

\begin{tabular}{|c|c|c|c|c|c|c|c|c|}
\hline $\begin{array}{l}\text { Station } \\
\text { number }\end{array}$ & Stream & Station location & $\begin{array}{l}\text { Drainage } \\
\text { area } \\
\left(\mathrm{mi}^{2}\right)\end{array}$ & Date & $\begin{array}{c}\text { Discharge } \\
\left(\mathrm{ft}^{3} / \mathrm{s}\right)\end{array}$ & $\begin{array}{ll}Q_{7,2} & Q_{7} \\
\left(f t^{3} / s\right) & \left(f^{3}\right.\end{array}$ & & $i^{a c y}$ \\
\hline TK7 & $\begin{array}{l}\text { West Twin River } \\
\text { tributary }\end{array}$ & $\begin{array}{l}\text { NE } 1 / 4 N W 1 / 4 \text { sec. } 7, T .20 \text {, N., R. } 23 \text { E., } \\
\text { Manitowoc County, at bridge on } \\
\text { County Trunk H, at Kellnersville, } \\
\text { Wis. }\end{array}$ & 2.25 & $\begin{array}{l}\text { Oct. } 11,1972 \\
\text { Aug. } 2,1973 \\
\text { Oct. } 21,1975 \\
\text { July } 13,1976\end{array}$ & $\begin{array}{l}20 \\
20 \\
20 \\
2\end{array}$ & 0 & 0 & c \\
\hline TK8 & $\begin{array}{l}\text { West Twin River } \\
\text { tributary }\end{array}$ & $\begin{array}{l}\text { NE } 1 / 4 S E 1 / 4 \text { sec. } 22, \text { T. } 21 \text { N., R. } 22 \text { E., } \\
\text { Manitowoc County, at bridge on } \\
\text { County Trunk A, at Maribel, Wis. }\end{array}$ & .82 & $\begin{array}{l}\text { Oct. } 11,1972 \\
\text { Aug. } 2,1973 \\
\text { Oct. } 21,1975 \\
\text { July } 13,1976\end{array}$ & $\begin{array}{l}2 \\
2 \\
2 \\
2 \\
2\end{array}$ & 0 & 0 & c \\
\hline TK9 & $\begin{array}{l}\text { West Twin River } \\
\text { tributary }\end{array}$ & $\begin{array}{l}\text { NW } 1 / 4 \text { SW } 1 / 4 \text { sec. } 30, \text { T. } 20 \text { N., R. } 23 \text { E., } \\
\text { Manitowoc County, at culvert on } \\
\text { country road, } 1.4 \text { mi northeast of } \\
\text { Rockwood, Wis. }\end{array}$ & 4.80 & $\begin{array}{l}\text { Oct. } 10,1972 \\
\text { July } 31,1973 \\
\text { Nov. } 14,1973 \\
\text { Oct. } 21,1975 \\
\text { July } 12,1976 \\
\text { Sept. } 14,1976 \\
\text { July } 25,1977\end{array}$ & $\begin{array}{l}1.39 \\
1.10 \\
1.36 \\
.64 \\
.16 \\
.80 \\
.49\end{array}$ & .27 & .16 & c \\
\hline \multirow[t]{2}{*}{ TK10 } & $\begin{array}{l}\text { West Twin River } \\
\text { tributary }\end{array}$ & $\begin{array}{l}\text { SE } 1 / 4 \text { SE } 1 / 4 \text { sec. } 14, \text { T. } 20 \text { N., R. } 23 \text { E., } \\
\text { Manitowoc County, at sewage-treatment } \\
\text { plant, } 0.9 \text { mi east of Francis Creek, } \\
\text { Wis. }\end{array}$ & .64 & & & & & \\
\hline & $\begin{array}{l}\text { West Twin River } \\
\text { tributary }\end{array}$ & $\begin{array}{l}\text { NE } 1 / 4 \text { NE } 1 / 4 \text { sec. } 24, T .20 \text { N., R. } 23 \text { E., } \\
\text { Manitowoc County, at culvert on } \\
\text { County Trunk Q, } 2.0 \text { mi east of } \\
\text { Francis Creek, Wis. }\end{array}$ & 2.45 & $\begin{array}{l}\text { July } 19,1972 \\
\text { Oct. } 11,1972 \\
\text { July } 31,1973 \\
\text { Oct. } 21,1975 \\
\text { July } 13,1976 \\
\text { Sept. } 14,1976 \\
\text { July } 25,1977\end{array}$ & $\begin{array}{l}.51 \\
.93 \\
.42 \\
.87 \\
.06 \\
.38 \\
.58\end{array}$ & $\underline{5}$ & $\underline{5}$ & \\
\hline TK12 & East Twin River & $\begin{array}{l}\text { SW } 1 / 4 \text { SE } 1 / 4 \text { sec. } 29, \text { T. } 22 \text { N., R. } 24 \text { E., } \\
\text { Kewaunee County, at bridge on } \\
\text { country road, } 1.2 \text { mi northeast of } \\
\text { Tisch Mills, Wis. }\end{array}$ & 50.2 & $\begin{array}{lc}\text { Oct. } & 11,1972 \\
\text { Aug. } & 2,1973 \\
\text { Nov. } 14,1973 \\
\text { Oct. } 21,1975 \\
\text { July } 13,1976 \\
\text { Sept. } 15,1976\end{array}$ & $\begin{array}{l}18.6 \\
10.1 \\
19.9 \\
9.06 \\
4.17 \\
3.22\end{array}$ & 3.1 & 1.9 & c \\
\hline \multirow[t]{2}{*}{ TK14 } & East Twin River & $\begin{array}{l}\text { SW } 1 / 4 \text { NW } 1 / 4 \text { sec. } 4, \text { T. } 20 \text { N., R. } 24 \text { E., } \\
\text { Manitowoc County, at State } \\
\text { Highway 147, at Mishicot, Wis. }\end{array}$ & 109 & $\begin{array}{l}\text { July } 19,1972 \\
\text { Oct. } 11,1972 \\
\text { July } 31,1973 \\
\text { Nov. } 14,1973 \\
\text { Oct. } 21,1975 \\
\text { July } 13,1976 \\
\text { Sept. } 14,1976\end{array}$ & $\begin{array}{c}314.5 \\
37.6 \\
15.8 \\
44.2 \\
16.1 \\
9.18 \\
5.06\end{array}$ & 7.4 & 4.4 & b \\
\hline & East Twin River & $\begin{array}{l}\text { NW } 1 / 4 N W 1 / 4 \text { sec. } 4, \text { T. } 20 \text { N., R. } 24 \text { E., } \\
\text { Manitowoc County, at sewage- } \\
\text { treatment plant, at Mishicot, Wis. }\end{array}$ & 111 & & & & & \\
\hline \multirow[t]{2}{*}{ TK17 } & Kewaunee River & $\begin{array}{l}\text { SE } 1 / 4 \text { SW } / 4 \text { sec. } 14, \text { T. } 23 \text { N., R. } 24 \text { E., } \\
\text { Kewaunee County, at County Trunk F, } \\
2.3 \text { mi west of Kewaunee, Wis. }\end{array}$ & 129 & & & 10 & 6.9 & $\mathbf{a}$ \\
\hline & Kewaunee River & $\begin{array}{l}\text { NE } 1 / 4 S E 1 / 4 \text { sec. } 18, \text { T. } 23 \text { N., R. } 25 \text { E., } \\
\text { Kewaunee County, at sewage- } \\
\text { treatment plant, at Kewaunee, Wis. }\end{array}$ & 137 & & & & & \\
\hline TK18 & Luxemburg Creek & $\begin{array}{l}\text { NW } / 4 \text { NE } 1 / 4 \text { sec. } 22, \text { T. } 24 \text { N., R. } 23 \text { E., } \\
\text { Kewaunee County, at bridge on } \\
\text { country road, } 0.7 \text { mi northeast } \\
\text { of Luxemburg, Wis. }\end{array}$ & 2.36 & $\begin{array}{lr}\text { July } 19,1972 \\
\text { Oct. } 11,1972 \\
\text { Oct. } 18,1972 \\
\text { Aug. } 1,1973 \\
\text { Oct. } 22,1975 \\
\text { July } 13,1976 \\
\text { Sept. } 15,1976\end{array}$ & $\begin{array}{l}.20 \\
.83 \\
.53 \\
.39 \\
.46 \\
.24 \\
.15\end{array}$ & .15 & .10 & $\mathbf{c}$ \\
\hline TK19 & Casco Creek & $\begin{array}{l}\text { NE } 1 / 4 N W 1 / 4 \text { sec. } 17 ., \text { T. } 24 \text { N., R. } 24 \text { E., } \\
\text { Kewaunee County, at bridge on County } \\
\text { Trunk C, at Casco, Wis. }\end{array}$ & 10.3 & $\begin{array}{l}\text { July } 19,1972 \\
\text { Oct. } 12,1972 \\
\text { Aug. } 1,1973 \\
\text { Oct. } 22,1975 \\
\text { July } 13,1976 \\
\text { Sept. } 15,1976\end{array}$ & $\begin{array}{l}3.62 \\
5.99 \\
3.83 \\
4.85 \\
1.19 \\
.56\end{array}$ & .60 & .26 & c \\
\hline
\end{tabular}


Table 27. Twin-Kewaunee River basin and Door County station locations and low-flow characteristics-Continued.

\begin{tabular}{|c|c|c|c|c|c|c|c|c|}
\hline $\begin{array}{l}\text { Station } \\
\text { number }\end{array}$ & 1 Stream & Station location & $\begin{array}{l}\text { Drainage } \\
\text { area } \\
\left(\mathrm{mi}^{2}\right)\end{array}$ & Date & $\begin{array}{c}\text { Discharge } \\
\left(\mathrm{ft}^{3} / \mathrm{s}\right)\end{array}$ & $\begin{array}{ll}\mathrm{Q}_{7,2} & \mathrm{Q}_{7} \\
\left(\mathrm{ft}^{3} / \mathrm{s}\right) & \left(\mathrm{ft}^{3}\right.\end{array}$ & ${ }_{1 / \mathrm{s})}^{10} \mathrm{~A}$ & $\begin{array}{c}\text { Accuracy } \\
\text { level }\end{array}$ \\
\hline TK25 & Maple Creek & $\begin{array}{l}\text { NE } 1 / 4 \text { NW } 1 / 4 \text { sec. } 19, \text { T. } 28 \text { N., R. } 27 \text { E., } \\
\text { Door County, at country road, } 1.0 \mathrm{mi} \\
\text { south of Valmy, Wis. }\end{array}$ & 6.75 & $\begin{array}{lc}\text { Oct. } & 2,1968 \\
\text { Oct. } & 13,1972 \\
\text { Aug. } & 1,1973 \\
\text { Nov. } 14,1973 \\
\text { Oct. } 23,1975 \\
\text { July } 12,1976\end{array}$ & $\begin{array}{l}{ }^{2} 0 \\
2.13 \\
2.61 \\
2_{0}^{.6} \\
2_{0}^{0}\end{array}$ & 0 & 0 & c \\
\hline TK27 & Ahnapee River & $\begin{array}{l}\text { NW1/4NE1/4 sec. } 32, \text { T. } 26 \text { N., R. } 25 \text { E., } \\
\text { Door County, at County Trunk J, } \\
0.3 \text { mi west of Forestville, Wis. }\end{array}$ & 32.3 & $\begin{array}{lr}\text { Oct. } & 3,1968 \\
\text { July } & 20,1972 \\
\text { Oct. } & 12,1972 \\
\text { Aug. } 1,1973 \\
\text { Oct. } 22,1975 \\
\text { July } 13,1976 \\
\text { Sept. } 17,1976\end{array}$ & $\begin{array}{l}5.70 \\
6.22 \\
25.2 \\
14.0 \\
4.88 \\
2.57 \\
1.71\end{array}$ & 1.8 & .90 & c \\
\hline TK29 & $\begin{array}{l}\text { Lake Michigan } \\
\text { tributary }\end{array}$ & $\begin{array}{l}\text { NE } 1 / 4 \text { NE } 1 / 4 \text { sec. } 20, \text { T. } 24 \text { N., R. } 25 \text { E., } \\
\text { Kewaunee County, at bridge on } \\
\text { country road, } 1.0 \text { mi east of } \\
\text { Alaska, Wis. }\end{array}$ & 1.53 & $\begin{array}{lr}\text { Oct. } & 12,1972 \\
\text { Aug. } & 1,1973 \\
\text { Nov. } 14,1973 \\
\text { Oct. } 22,1975 \\
\text { July } 14,1976 \\
\text { Sept. } 15,1976\end{array}$ & $\begin{array}{l}.74 \\
.03 \\
.34 \\
.13 \\
20\end{array}$ & 0 & 0 & c \\
\hline TK1A & Kewaunee River & $\begin{array}{l}\text { NW1/4NW1/4 sec. } 13, \text { T. } 24 \text { N., R. } 23 \text { E., } \\
\text { Kewaunee County, at bridge on State } \\
\text { Highway } 54,2.1 \text { mi west of Casco, }\end{array}$ & 65.6 & $\begin{array}{l}\text { Oct. } 22,1975 \\
\text { July } 14,1976 \\
\text { July } 26,1977\end{array}$ & $\begin{array}{l}6.40 \\
.66 \\
.77\end{array}$ & .81 & .35 & c \\
\hline TK2A S & School Creek & $\begin{array}{l}\text { NE } 1 / 4 S E 1 / 4 \text { sec. } 10, \text { T. } 24 \text { N., R. } 23 \text { E., } \\
\text { Kewaunee County, at bridge on } \\
\text { country road, } 2.0 \text { mi northeast of } \\
\text { Luxemburg, Wis. }\end{array}$ & 24.6 & $\begin{array}{l}\text { Oct. } 22,1975 \\
\text { July } 13,1976 \\
\text { Sept. } 15,1976 \\
\text { July } 26,1977\end{array}$ & $\begin{array}{l}.92 \\
.08 \\
.05 \\
.12\end{array}$ & .05 & .01 & c \\
\hline TK3A & Silver Creek & $\begin{array}{l}\text { NE } 1 / 4 \text { SW } 1 / 4 \text { sec. } 28, \text { T. } 25 \text { N., R. } 25 \text { E., } \\
\text { Kewaunee County, at bridge on } \\
\text { country road, } 1.7 \text { mi west of } \\
\text { post office in Algoma, Wis. }\end{array}$ & 61.3 & $\begin{array}{l}\text { Oct. } 22,1975 \\
\text { July } 13,1976 \\
\text { Sept. } 15,1976 \\
\text { July } 26,1977\end{array}$ & $\begin{array}{l}4.80 \\
.08 \\
.16 \\
.06\end{array}$ & .08 & .01 & c \\
\hline
\end{tabular}

1 Based on 1971 report by Wisconsin Department of Natural Resources (DNR). Site lettered "A" was not included in 1971 DNR report.

${ }^{2}$ Streambed is dry.

3 Additional discharge measurements are available.

${ }^{5}$ No estimate possible--discharge is primarily effluent. 
Table 28. Lower Wisconsin River basin station locations and low-flow characteristics.

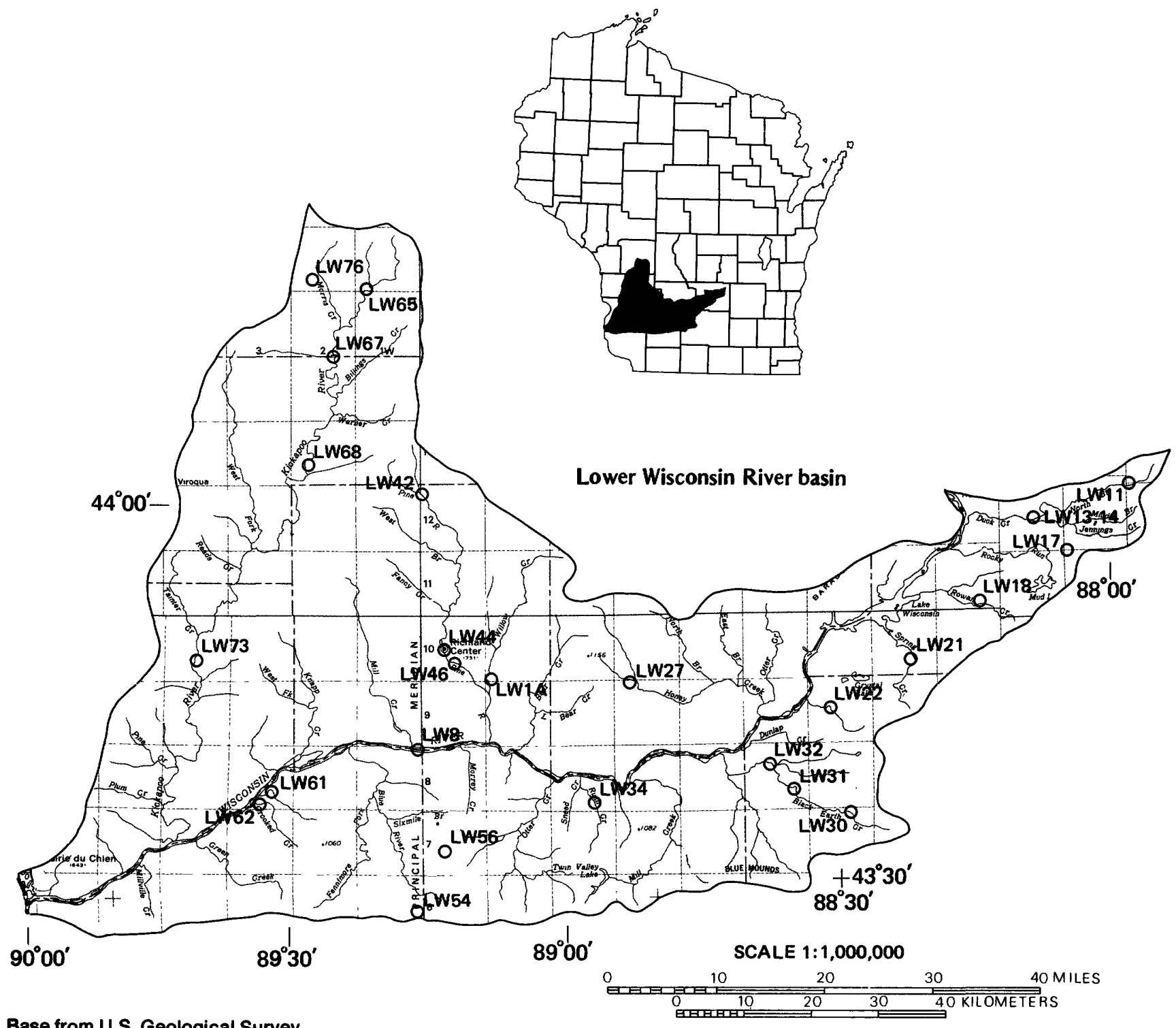

Base from U.S. Geological Survey

State base map, 1968

\begin{tabular}{|c|c|c|c|c|c|c|c|c|}
\hline $\begin{array}{l}\text { Station } \\
\text { number }\end{array}$ & Stream & Station location & $\begin{array}{l}\text { Drainage } \\
\text { area } \\
\left(\mathrm{mi}^{2}\right)\end{array}$ & Date & $\begin{array}{c}\text { Discharge } \\
\left(\mathrm{ft}^{3} / \mathrm{s}\right)\end{array}$ & $\begin{array}{l}\mathrm{Q}_{7,2} \mathrm{Q}_{7} \\
\left(\mathrm{ft}^{3} / \mathrm{s}\right)\left(\mathrm{ft}^{2}\right.\end{array}$ & $\begin{array}{l}7,10 \\
3 / \mathrm{s})\end{array}$ & $\underset{\text { Aevel }}{\text { Accuracy }}$ \\
\hline LW8 & Wisconsin River & $\begin{array}{l}\text { SE } 1 / 4 \text { NW } 1 / 4 \text { sec. } 1, \text { T. } 8 \text { N., R. } 1 \text { W., } \\
\text { Grant County, on left bank at } \\
\text { bridge on State Highway } 80 \text {, } \\
0.5 \text { mi upstream from Eagle Mill } \\
\text { Creek, } 1.0 \text { mi north of Muscoda, } \\
\text { Wis. }\end{array}$ & 10,450 & - & & 3,7702 , & 790 & a \\
\hline LW11 & $\begin{array}{l}\text { North Branch } \\
\text { Duck Creek }\end{array}$ & $\begin{array}{l}\text { NE } 1 / 4 N W 1 / 4 \text { sec. } 6, T .12 \text { N., R. } 12 \text { E., } \\
\text { Columbia County, at bridge on } \\
\text { town road, } 0.1 \text { mi downstream from } \\
\text { sewage-treatment plant, } 0.6 \mathrm{mi} \\
\text { west of Cambria, Wis. }\end{array}$ & 8.55 & $\begin{array}{l}\text { July } 5,1972 \\
\text { June } 25,1973 \\
\text { Oct. } 10,1975 \\
\text { Sept. } 5,1978\end{array}$ & $\begin{array}{l}{ }^{4} 0 \\
8.50 \\
.96 \\
.86\end{array}$ & $<.01$ & $<.01$ & c \\
\hline $\begin{array}{l}\text { LW13, } \\
14\end{array}$ & Duck Creek & $\begin{array}{l}\text { NE } 1 / 4 N W 1 / 4 \text { sec. } 21, \text { T. } 12 \text { N., R. } 10 \text { E., } \\
\text { Columbia County, at bridge in } \\
\text { park, at Wyocena, Wis. }\end{array}$ & 79.6 & $\begin{array}{ll}\text { July } & 5,1972 \\
\text { June } & 25,1973 \\
\text { Aug. } & 21,1973 \\
\text { Oct. } & 10,1975\end{array}$ & $\begin{array}{l}20.7 \\
43.3 \\
35.3 \\
17.9\end{array}$ & 15 & 12 & c \\
\hline
\end{tabular}


Table 28. Lower Wisconsin River basin station locations and low-flow characteristics-Continued.

\begin{tabular}{|c|c|c|c|c|c|c|c|c|}
\hline $\begin{array}{l}\text { Station } \\
\text { number }\end{array}$ & ${ }^{1}$ Stream & Station location & $\begin{array}{l}\text { Drainage } \\
\text { area } \\
\left(\mathrm{mi}^{2}\right)\end{array}$ & Date & $\begin{array}{c}\text { Discharge } \\
\left(\mathrm{ft}^{3} / \mathrm{s}\right)\end{array}$ & $\begin{array}{l}\mathrm{Q}_{7,2} \mathrm{Q}_{7} \\
\left(\mathrm{ft}^{3} / \mathrm{s}\right)\left(\mathrm{ft}^{3}\right.\end{array}$ & $\begin{array}{l}7,10 \\
3 / \mathrm{s})\end{array}$ & $\begin{array}{c}\text { Accuracy } \\
\text { level }\end{array}$ \\
\hline LW17 & $\begin{array}{l}\text { Rocky Run } \\
\text { tributary }\end{array}$ & $\begin{array}{l}\text { SE } 1 / 4 N E 1 / 4 \text { sec. } 1, T .11 \text { N., R. } 10 \text { E., } \\
\text { Columbia County, at sewage- } \\
\text { treatment plant, } 0.5 \text { mi northwest } \\
\text { of Rio, Wis. }\end{array}$ & 1.55 & $\begin{array}{l}\text { July } 5,1972 \\
\text { June } 25,1973 \\
\text { Aug. } 21,1973 \\
\text { Oct. } 10,1975\end{array}$ & $\begin{array}{l}{ }_{20} \\
{ }_{0}^{5.57} \\
2_{0}^{5.57}\end{array}$ & 0 & 0 & c \\
\hline LW18 & Rowan Creek & $\begin{array}{l}\text { NE } 1 / 4 \text { SW } 1 / 4 \text { sec. } 35, \text { T. } 11 \text { N., R. } 9 \text { E., } \\
\text { Columbia County, at bridge on } \\
\text { U.S. Highway } 51 \text {, in Poynette, Wis. }\end{array}$ & 10.4 & $\begin{array}{l}\text { Oct. } 10,1975 \\
\text { Aug. } 3,1977\end{array}$ & $\begin{array}{l}{ }^{3} 6.22 \\
6.95\end{array}$ & 3.6 & 2.8 & b \\
\hline LW21 & Spring Creek & $\begin{array}{l}\text { SE1//4NE } 1 / 4 \text { sec. } 27, T .10 \text { N., R. } 8 \text { E., } \\
\text { Columbia County, at bridge on } \\
\text { town road, in Lodi, Wis. }\end{array}$ & 37.0 & & & 16 & 15 & b \\
\hline LW22 & Blums Creek & $\begin{array}{l}\text { SW } 1 / 4 \text { SW } 1 / 4 \text { sec. } 16, \text { T. } 9 \text { N., R. } 7 \text { E., } \\
\text { Dane County, at bridge on town } \\
\text { road, in Roxbury, Wis. }\end{array}$ & 9.27 & $\begin{array}{l}\text { July } 5,1972 \\
\text { June } 25,1973 \\
\text { Aug. } 21,1973 \\
\text { Oct. } 9,1975\end{array}$ & $\begin{array}{l}.03 \\
.88 \\
.90 \\
.82\end{array}$ & .01 & $<.01$ & c \\
\hline LW27 & $\begin{array}{l}\text { Honey Creek } \\
\text { tributary }\end{array}$ & $\begin{array}{l}\text { SE } 1 / 4 \text { SE } 1 / 4 \text { sec. } 5, \text { T. } 9 \text { N., R. } 4 \text { E., } \\
\text { Sauk County, at sewage-treatment } \\
\text { plant about } 600 \text { ft downstream } \\
\text { from State Highway } 23 \text { bridge, } \\
\text { in Plain, Wis. }\end{array}$ & 6.59 & $\begin{array}{l}\text { July } 7,1972 \\
\text { June } 26,1973 \\
\text { Aug. } 22,1973 \\
\text { Oct. } 7,1975\end{array}$ & $\begin{array}{l}.69 \\
1.61 \\
1.38 \\
.99\end{array}$ & .56 & .41 & c \\
\hline LW30 & $\begin{array}{l}\text { Black Earth } \\
\text { Creek }\end{array}$ & $\begin{array}{l}\text { SW } 1 / 4 \text { NW } 1 / 4 \text { sec. } 3, \text { T. } 7 \text { N., R. } 7 \text { E., } \\
\text { Dane County, at bridge on County } \\
\text { Trunk KP, in Cross Plains, Wis. }\end{array}$ & 26.1 & $\begin{array}{l}\text { July } 7,1972 \\
\text { June } 25,1973 \\
\text { July } 27,1973 \\
\text { Aug. } 22,1973 \\
\text { Dec. } 12,1973 \\
\text { Oct. } 9,1975\end{array}$ & $\begin{array}{l}7.63 \\
19.6 \\
19.6 \\
14.8 \\
15.3 \\
13.8\end{array}$ & 6.0 & 4.3 & c \\
\hline \multirow[t]{2}{*}{ LW31 } & $\begin{array}{l}\text { Black Earth } \\
\text { Creek }\end{array}$ & $\begin{array}{l}\text { SE } 1 / 4 \text { SW } 1 / 4 \text { sec. } 25, \text { T. } 8 \text { N., R. } 6 \text { E., } \\
\text { Dane County, } 100 \text { ft upstream from } \\
\text { unnamed tributary, } 0.7 \text { mi east of } \\
\text { Black Earth, Wis. }\end{array}$ & 45.6 & & & 17 & 13 & a \\
\hline & $\begin{array}{l}\text { Black Earth } \\
\text { Creek }\end{array}$ & $\begin{array}{l}\text { SE } 1 / 4 \text { NW1/4 sec. } 26 \text {, T. } 8 \text { N., R. } 6 \text { E., } \\
\text { Dane County, at bridge on U.S. } \\
\text { Highway } 14 \text { and State Highway } 78, \\
200 \mathrm{ft} \text { upstream from sewage- } \\
\text { treatment plant, at Black Earth, } \\
\text { Wis. }\end{array}$ & 48.3 & & & & & \\
\hline LW32 & $\begin{array}{l}\text { Black Earth } \\
\text { Creek }\end{array}$ & $\begin{array}{l}\text { SW } 1 / 4 \text { SW } 1 / 4 \text { sec. 9, T. } 8 \text { N., R. } 6 \text { E., } \\
\text { Dane County, at bridge on Bridge } \\
\text { Street, at Mazomanie, Wis. }\end{array}$ & 99.0 & $\begin{array}{l}\text { July } 7,1972 \\
\text { June } 26,1973 \\
\text { July } 27,1973 \\
\text { Aug. } 21,1973 \\
\text { Dec. } 12,1973 \\
\text { Oct. } 9,1975\end{array}$ & $\begin{array}{l}28.0 \\
76.8 \\
67.0 \\
60.7 \\
56.8 \\
39.3\end{array}$ & 22 & 15 & c \\
\hline LW34 & Rush Creek & $\begin{array}{l}\text { SW } 1 / 4 \text { NW } 1 / 4 \text { sec. } 35, \text { T. } 8 \text { N., R. } 3 \text { E., } \\
\text { lowa County, at bridge on State } \\
\text { Highway } 23,4.2 \text { mi southwest of } \\
\text { Spring Green, Wis. }\end{array}$ & 13.9 & $\begin{array}{l}\text { Aug. } 19,1970 \\
\text { June } 21,1977 \\
\text { Sept. } 5,1978\end{array}$ & $\begin{array}{l}3.18 \\
3.24 \\
5.81\end{array}$ & 3.0 & 2.0 & c \\
\hline LW42 & Pine River & $\begin{array}{l}\text { SW } 1 / 4 \text { SW } 1 / 4 \text { sec. } 6, \text { T. } 12 \text { N., R. } 1 \text { E., } \\
\text { Richland County, at bridge on } \\
\text { State Highway } 80 \text {, at Yuba, Wis. }\end{array}$ & 27.2 & $\begin{array}{l}\text { July } 6,1972 \\
\text { Oct. } 17,1972 \\
\text { June } 26,1973 \\
\text { Aug. } 22,1973 \\
\text { June } 3,1974 \\
\text { Sept. } 9,1974 \\
\text { Oct. } 9,1975 \\
\text { May } 15,1975\end{array}$ & $\begin{array}{l}5.27 \\
12.9 \\
19.8 \\
14.9 \\
18.6 \\
10.5 \\
10.7 \\
16.8\end{array}$ & 3.7 & 2.5 & c \\
\hline LW44 & Pine River & $\begin{array}{l}\text { SW } 1 / 4 \text { NW } 1 / 4 \text { sec. } 21, \text { T. } 10 \text { N., R. } 1 \text { E., } \\
\text { Richland County, at sewage- } \\
\text { treatment plant, at Richland } \\
\text { Center, Wis. }\end{array}$ & 190 & $\begin{array}{l}\text { Oct. } 18,1972 \\
\text { June } 27,1973 \\
\text { Aug. } 22,1973 \\
\text { Oct. } 7,1975\end{array}$ & $\begin{array}{l}110 \\
189 \\
129 \\
87.6\end{array}$ & 65 & 55 & c \\
\hline
\end{tabular}


Table 28. Lower Wisconsin River basin station locations and low-flow characteristics-Continued.

\begin{tabular}{|c|c|c|c|c|c|c|c|c|}
\hline \multirow{2}{*}{$\begin{array}{l}\text { Station } \\
\text { number } \\
\text { LW46 P }\end{array}$} & \multirow{2}{*}{$\begin{array}{l}\text { Stream } \\
\text { name }\end{array}$} & \multirow{2}{*}{$\begin{array}{l}\text { Station location } \\
\text { SE1/4SE1/\% sec. } 27, T \text {. } 10 \mathrm{~N} \text {., R. } 1 \text { E., } \\
\text { Richland County, at bridge on } \\
\text { County Trunk O, } 2.5 \mathrm{mi} \text { southeast } \\
\text { of Richland Canter, Wis. }\end{array}$} & \multirow{2}{*}{$\begin{array}{c}\begin{array}{c}\text { Drainage } \\
\text { area } \\
\left(\mathrm{mi}^{2}\right)\end{array} \\
198\end{array}$} & \multirow{2}{*}{ 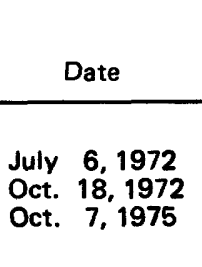 } & \multirow{2}{*}{$\begin{array}{l}\text { Discharge } \\
\left(\mathrm{ft}^{3} / \mathrm{s}\right)\end{array}$} & \multicolumn{3}{|c|}{$\begin{array}{l}\mathrm{Q}_{7,2} \mathrm{Q}_{7,10} \\
\left(\mathrm{ft}^{3} / \mathrm{s}\right)\left(\mathrm{ft}^{3} / \mathrm{s}\right)\end{array}$} \\
\hline & & & & & & 74 & 63 & b \\
\hline & Pine River & $\begin{array}{l}\text { NE1/\%WW1/ sec. 35, T. } 10 \text { N., R. } 1 \text { E., } \\
\text { Richland County, at sewage- } \\
\text { traatment plant outfall at County } \\
\text { Home, near Richland Center, Wis. }\end{array}$ & 202 & & & & & \\
\hline LW54 & $\begin{array}{l}\text { Blue River } \\
\text { tributary }\end{array}$ & $\begin{array}{l}\text { NE } 1 / 4 \text { SW } 1 / 2 \text { sec. } 24, \text { T. } 6 \text { N., R. } 1 \text { W., } \\
\text { Grant County, at sewage-treatment } \\
\text { plant, in Montfort, Wis. }\end{array}$ & .58 & $\begin{array}{l}\text { July } 7,1972 \\
\text { June } 28,1973 \\
\text { Aug. } 23,1973 \\
\text { Oct. } 8,1975\end{array}$ & $\begin{array}{l}.55 \\
1.26 \\
1.12 \\
.64\end{array}$ & .36 & .26 & c \\
\hline LW56 & $\begin{array}{l}\text { Big Spring } \\
\text { Branch }\end{array}$ & $\begin{array}{l}\text { NE1/2SE1// sec. } 29, \text { T. } 7 \text { N., R. } 1 \text { E., } \\
\text { lowa County, at sewage-disposal } \\
\text { pond, at Highland, Wis. }\end{array}$ & .68 & $\begin{array}{l}\text { Aug. } 23,1973 \\
\text { Oct. } 8,1975 \\
\text { Sept. } 5,1978\end{array}$ & $\begin{array}{r}.30 \\
<.01 \\
.03\end{array}$ & .01 & $<.01$ & c \\
\hline LW61 & Saunders Creek & $\begin{array}{l}\text { SE } 1 / 4 N E 1 / 4 \text { sec. } 27, \text { T. } 8 \text { N., R. } 3 \text { W., } \\
\text { Crawford County, at sewage- } \\
\text { treatment plant, in Boscobel, Wis. }\end{array}$ & 17.1 & $\begin{array}{l}\text { July } 6,1972 \\
\text { June } 27,1973 \\
\text { Aug. } 23,1973 \\
\text { Oct. } 8,1975\end{array}$ & $\begin{array}{c}4.88 \\
13.9 \\
12.5 \\
6.91\end{array}$ & 3.4 & 2.4 & c \\
\hline LW62 & Crooked Creek & NW $1 / 4$ NE $1 / 4$ sec. 4, T. 7 N., R. 3 W., & 16.7 & Oct. 8,1975 & 10.1 & $6 . .6$ & 5.3 & c \\
\hline & & $\begin{array}{l}\text { Grant County, at bridge on State } \\
\text { Highway } 132,1.6 \text { mi southwest of } \\
\text { Boscobel, Wis. }\end{array}$ & & $\begin{array}{l}\text { June } 21,1977 \\
\text { Sept. 5, } 1978\end{array}$ & $\begin{array}{l}7.09 \\
6.94\end{array}$ & & & \\
\hline LW65 & Kickapoo River & $\begin{array}{l}\text { NW1/4NW1/4 sec. } 6, \text { T. } 15 \mathrm{~N} ., \mathrm{R} .1 \mathrm{~W} \text {., } \\
\text { Monroe County, at bridge on State } \\
\text { Highway } 131,500 \mathrm{ft} \text { upstream from } \\
\text { sewage-treatment plant, at Wilton, } \\
\text { Wis. }\end{array}$ & 34.9 & $\begin{array}{l}\text { Aug. } 18,1970 \\
\text { July } 6,1972 \\
\text { June } 26,1973 \\
\text { Oct. } 9,1975 \\
\text { Sept. } 8,1977\end{array}$ & $\begin{array}{c}7.88 \\
9.66 \\
25.8 \\
13.5 \\
7.26\end{array}$ & 6.6 & 4.8 & c \\
\hline LW67 & Kickapoo River & $\begin{array}{l}\text { SW1/4NW1/4 sec. } 2, T .14 \text { N., R. } 2 \text { W., } \\
\text { Vernon County, at bridge on State } \\
\text { Highway } 33,0.1 \text { mi upstream from } \\
\text { sewage-treatment plant, in } \\
\text { Ontario, Wis. }\end{array}$ & 117 & $\begin{array}{l}\text { Aug. } 26,1969 \\
\text { Sept. } 29,1969 \\
\text { Aug. } 18,1970 \\
\text { Dec. } 7,1972 \\
\text { June } 26,1973\end{array}$ & $\begin{array}{l}34.0 \\
55.4 \\
29.2 \\
45.5 \\
86.0\end{array}$ & 28 & 21 & c \\
\hline LW68 & Kickapoo River & $\begin{array}{l}\mathrm{SE} 1 / 4 \mathrm{NW} 1 / 4 \text { sec. } 29, \mathrm{~T} .13 \mathrm{~N} ., \mathrm{R} .2 \mathrm{~W} \text {., } \\
\text { Vernon County, on left bank } 10 \mathrm{ft} \\
\text { upstream from bridge on State } \\
\text { Highway } 82 \text {, in La Farge, } 0.3 \mathrm{mi} \\
\text { upstream from Otter Creek, } 1.0 \mathrm{mi} \\
\text { downstream from powerplant. }\end{array}$ & 266 & & & 77 & 63 & a \\
\hline LW73 & Kickapoo River & $\begin{array}{l}\text { NE1/4 sec. } 28, \text { T. } 10 \text { N., R. } 4 \text { W., } \\
\text { Crawford County, on upstream side } \\
\text { of State Highway } 171 \text { bridge, } 300 \mathrm{ft} \\
\text { downstream from dam in Gays Mills, } \\
3.3 \text { mi downstream from Tainter } \\
\text { Creek. }\end{array}$ & 617 & & & 203 & 153 & $\mathbf{a}$ \\
\hline LW76 & $\begin{array}{l}\text { Morris Creek } \\
\text { tributary }\end{array}$ & $\begin{array}{l}\text { SW1/4SE1/4 sec. } 28, \text { T. } 16 \text { N., R. } 2 \text { W., } \\
\text { Monroe County, at sewage-treatment } \\
\text { plant, } 400 \text { ft downstream from } \\
\text { County Highway U bridge, in } \\
\text { Norwalk, Wis. }\end{array}$ & 19.9 & $\begin{array}{l}\text { July } 6,1972 \\
\text { June } 26,1973 \\
\text { Aug. } 23,1973 \\
\text { Oct. } 9,1975 \\
\text { Sept. } 8,1977\end{array}$ & $\begin{array}{c}4.98 \\
8.82 \\
19.2 \\
4.77 \\
3.17\end{array}$ & 2.8 & 1.8 & c \\
\hline
\end{tabular}


Table 28. Lower Wisconsin River basin station locations and low-flow characteristics-Continued.

\begin{tabular}{|c|c|c|c|c|c|c|c|}
\hline $\begin{array}{l}\text { Station } \\
\text { number }\end{array}$ & Station location & $\begin{array}{c}\text { Drainage } \\
\text { area } \\
\left(\mathrm{mi}^{2}\right)\end{array}$ & Date & $\begin{array}{c}\text { Discharge } \\
\left(\mathrm{ft}^{3} / \mathrm{s}\right)\end{array}$ & $\begin{array}{l}\mathrm{a}_{7,2} \\
\left.\mathrm{ift}^{3} / \mathrm{s}\right)\end{array}$ & $\begin{array}{l}0_{7,10} \\
\left(\mathrm{ft}^{3} / \mathrm{s}\right)\end{array}$ & Accuracy \\
\hline LW1A Willow Creek & $\begin{array}{l}\text { SEYSWY sec. 6, T. } 9 \text { N., R. } 2 \text { E., } \\
\text { Richland County, } 100 \mathrm{ft} \text { upstream } \\
\text { from bridge on U.S. Highway } 14 \text {, } \\
2.0 \text { mi southeast of State Highway } \\
58,2.3 \text { mi north of Gotham, } 6 \text { mi } \\
\text { southeast of Richland Center, Wis. }\end{array}$ & 82.5 & $\begin{array}{ll}\text { Oct. } & 7,1975 \\
\text { May } & 28,1976\end{array}$ & $\begin{array}{r}340.5 \\
45.6\end{array}$ & 31 & 27 & b \\
\hline
\end{tabular}

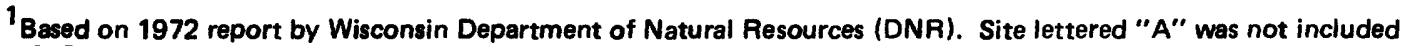
in 1972 DNR report.

${ }^{2}$ Streambed is dry.

${ }^{3}$ Additional discharge measurements are available.

${ }^{4}$ Negligible discharge--water ponded, unable to measure velocity. 
Table 29. Upper Wisconsin River basin station locations and low-flow characteristics.

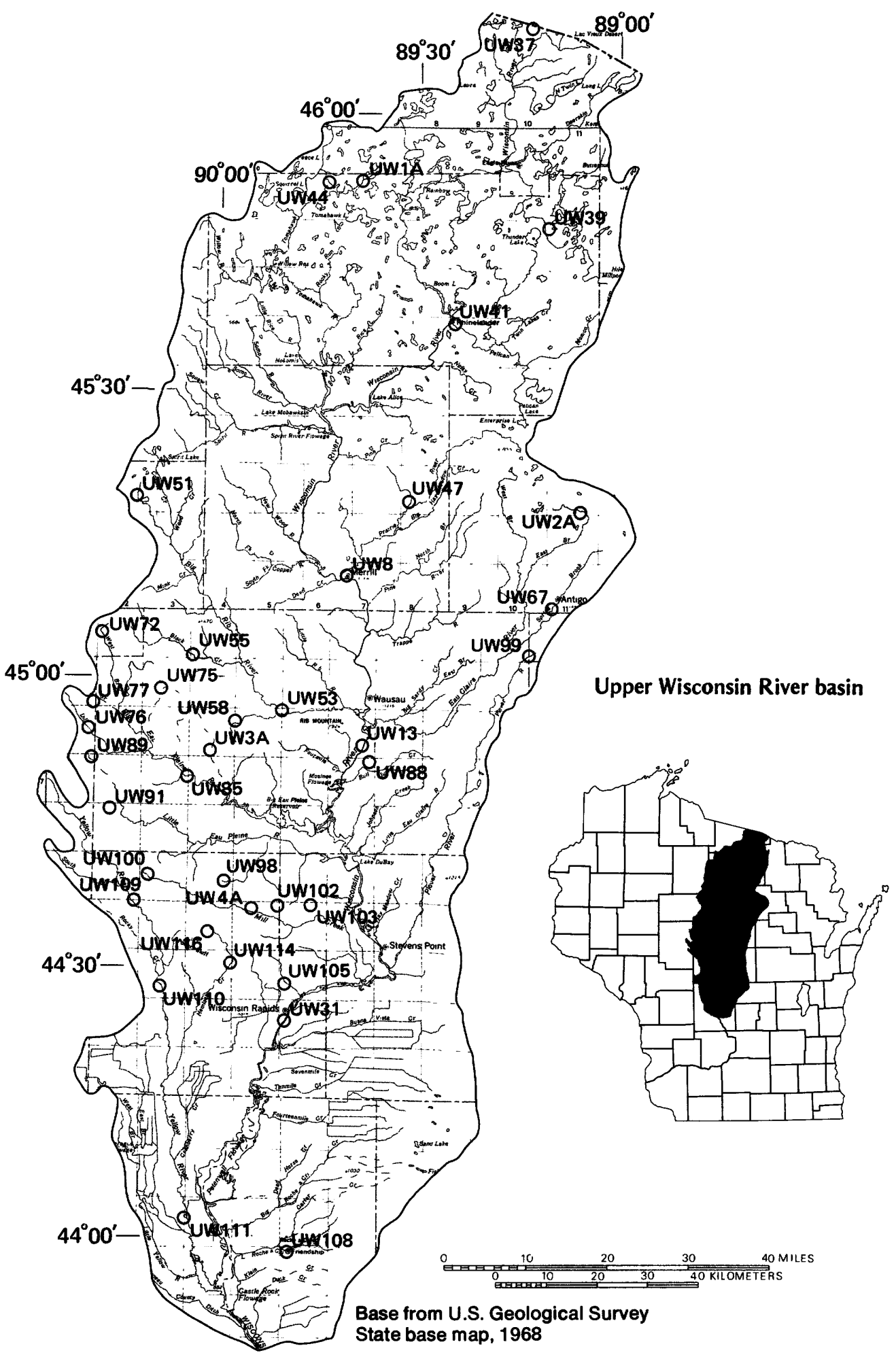


Table 29. Upper Wisconsin River basin station locations and low-flow characteristics-Continued.

\begin{tabular}{|c|c|c|c|c|c|c|c|c|}
\hline $\begin{array}{l}\text { Station } \\
\text { number }\end{array}$ & $n^{1} \underset{\text { name }}{\text { Stream }}$ & Station location & $\begin{array}{l}\text { Drainage } \\
\text { area } \\
\left(m i^{2}\right)\end{array}$ & Date & $\begin{array}{l}\text { Discharge } \\
\left(\mathrm{ft}^{3} / \mathrm{s}\right)\end{array}$ & $\begin{array}{l}0_{7,2} \\
\left(\mathrm{ft}^{3} / \mathrm{s}\right)(\end{array}$ & $\begin{array}{l}Q_{7,10} \\
\left(\mathrm{ft}^{3} / \mathrm{s}\right)\end{array}$ & $\begin{array}{c}\text { Accuracy } \\
\text { level }\end{array}$ \\
\hline UW8 & Wisconsin River & $\begin{array}{l}\text { SW1/4SE1/4 sec. 12, T. } 31 \text { N., R. } 6 \text { E., } \\
\text { Lincoln County, on left bank } \\
300 \mathrm{ft} \text { downstream from highway } \\
\text { bridge at east end of Merrill, } \\
0.5 \mathrm{mi} \text { downstream from Prairie } \\
\text { River. }\end{array}$ & 2,760 & & & 1,330 & 880 & $\mathbf{a}$ \\
\hline UW13 & Wisconsin River & $\begin{array}{l}\text { NW1/4NE } 1 / 4 \text { sec. } 26, \text { T. } 28 \text { N., R. } 7 \text { E., } \\
\text { Marathon County, on left bank at } \\
\text { Rothschild, } 0.5 \text { mi downstream } \\
\text { from Rothschild Dam, } 2.0 \text { mi } \\
\text { downstream from Eau Claire River, } \\
5.0 \text { mi upstream from Black Creek, } \\
5.5 \text { mi south of bridge on State } \\
\text { Highway } 29, \text { in Wausau, Wis. }\end{array}$ & 4,020 & & & 1,460 & 950 & $\mathbf{a}$ \\
\hline UW31 & Wisconsin River & $\begin{array}{l}\text { SW1/4 sec. } 24, T .22 \text { N., R. } 5 \text { E., } \\
\text { Wood County, at Centralia power- } \\
\text { plant of Nekoosa-Edwards Paper } \\
\text { Company, } 1.6 \text { mi downstream from } \\
\text { Chicago and NorthWestern Railroad } \\
\text { bridge, in Wisconsin Rapids, Wis. }\end{array}$ & 5,430 & & & 1,870 & 1,280 & $\mathbf{a}$ \\
\hline UW37 & $\begin{array}{l}\text { Wisconsin River } \\
\text { tributary }\end{array}$ & $\begin{array}{l}\text { SW1/4SW1/4 sec. } 35, \text { T. } 43 \text { N., R. } 10 \text { E., } \\
\text { Vilas County, just upstream from } \\
\text { sewage-treatment plant, at Land } \\
O^{\prime} \text { Lakes, Wis. }\end{array}$ & .44 & $\begin{array}{l}\text { July } 12,1972 \\
\text { June } 20,1973 \\
\text { July } 23,1974 \\
\text { July } 15,1975 \\
\text { Aug. } 1,1975\end{array}$ & $\begin{array}{l}0.23 \\
4_{0}^{.30} \\
4_{0}^{0}\end{array}$ & 0 & 0 & c \\
\hline UW39 & Townline Creek & $\begin{array}{l}\text { SE } 1 / 4 N E 1 / 4 \text { sec. 1, T. } 38 \text { N., R. } 10 \text { E., } \\
\text { Oneida County, at U.S. Highway } 45, \\
0.8 \text { mi north of Three Lakes, Wis. }\end{array}$ & 3.29 & $\begin{array}{l}\text { July } 13,1972 \\
\text { Dec. } 1,1972 \\
\text { June } 21,1973 \\
\text { Aug. } 30,1973 \\
\text { July } 15,1975 \\
\text { Aug. } 1,1975\end{array}$ & $\begin{array}{l}{ }^{4} 0 \\
2.61 \\
{ }_{4}^{4} 0^{0} \\
{ }_{4}^{0}\end{array}$ & 0 & 0 & c \\
\hline UW41 & Pelican River & $\begin{array}{l}\text { NW1/4NW\%/4 sec. } 8, \text { T. } 36 \text { N., R. } 9 \text { E., } \\
\text { Oneida County, at bridge on } \\
\text { County Trunk G, at Rhinelander, } \\
\text { Wis. }\end{array}$ & 264 & $\begin{array}{lc}\text { July } & 13,1972 \\
\text { June } 21,1973 \\
\text { Aug. } 30,1973 \\
\text { Oct. } 3,1973 \\
\text { July } 16,1975 \\
\text { Aug. } 2,1975 \\
\text { Aug. } 19,1975 \\
\text { Jan. } 16,1976 \\
\text { Sept. } 16,1976\end{array}$ & $\begin{array}{c}75.2 \\
145 \\
153 \\
183 \\
55.6 \\
22.4 \\
20.6 \\
143 \\
6.76\end{array}$ & 19 & 10 & c \\
\hline UW44 & Tomahawk River & $\begin{array}{l}\text { NW1/4SE } 1 / 4 \text { sec. } 9, \text { T. } 39 \text { N., R. } 6 \text { E., } \\
\text { Oneide County, at bridge on State } \\
\text { Highwey } 70,1.8 \text { mi west of } \\
\text { Minocqua, Wis. }\end{array}$ & 74.2 & $\begin{array}{l}\text { June } 28,1971 \\
\text { Oct. } 11,1971 \\
\text { July } 11,1972 \\
\text { June } 20,1973 \\
\text { Aug. } 29,1973 \\
\text { July } 25,1974 \\
\text { July } 14,1975 \\
\text { Aug. } 1,1975\end{array}$ & $\begin{array}{c}8.56 \\
121^{-1} \\
8.16 \\
138 \\
59.4 \\
14.7 \\
67.5 \\
10.0\end{array}$ & $\underline{6}$ & $\underline{6}$ & \\
\hline UW47 & $\begin{array}{l}\text { North Branch } \\
\text { Prairie River }\end{array}$ & $\begin{array}{l}\text { NE } 1 / 4 N E 1 / 4 \text { sec. } 19, \text { T. } 33 \text { N., R. } 8 \text { E., } \\
\text { Lincoln County, at bridge on } \\
\text { Axen Road, } 2.2 \text { mi north of } \\
\text { Gleason, Wis. }\end{array}$ & 34.8 & $\begin{array}{l}\text { Oct. } 12,1971 \\
\text { July } 10,1972 \\
\text { June } 21,1973 \\
\text { Aug. } 28,1973 \\
\text { Oct. } 3,1973 \\
\text { Julv } 15,1975 \\
\text { Aug. } 2,1975 \\
\text { Sept. } 16,1976\end{array}$ & $\begin{array}{l}16.7 \\
14.0 \\
22.5 \\
15.7 \\
21.1 \\
26.0 \\
11.1 \\
12.9\end{array}$ & 12 & 9.4 & c \\
\hline UW51 & $\begin{array}{l}\text { Sheep Ranch } \\
\text { Creek }\end{array}$ & $\begin{array}{l}\text { SWY/4NE1/4 sec. } 27, \text { T. } 33 \text { N., R. } 2 \text { E., } \\
\text { Taylor County, at culvert on private } \\
\text { road, at Rib Lake, Wis. }\end{array}$ & 7.84 & $\begin{array}{l}\text { Sept. } 6,1972 \\
\text { July } 11,1973 \\
\text { Aug. } 15,1973 \\
\text { July } 31,1975 \\
\text { July } 8,1976 \\
\text { Sept. } 15,1976\end{array}$ & $\begin{array}{l}1.49 \\
.87 \\
1.32 \\
.21 \\
.51 \\
.25\end{array}$ & .43 & .17 & c \\
\hline
\end{tabular}


Table 29. Upper Wisconsin River basin station locations and low-flow characteristics-Continued.

\begin{tabular}{|c|c|c|c|c|c|c|c|c|}
\hline $\begin{array}{l}\text { Station } \\
\text { number }\end{array}$ & ${ }_{r}^{1}$ Stream & Station location & $\begin{array}{l}\text { Drainage } \\
\text { area } \\
\left(\mathrm{mi}^{2}\right)\end{array}$ & Date & $\begin{array}{c}\text { Discharge } \\
\left(\mathrm{ft}^{3} / \mathrm{s}\right)\end{array}$ & $\begin{array}{l}\mathrm{Q}_{7,2} \mathrm{Q}_{7} \\
\left(\mathrm{ft}^{3} / \mathrm{s}\right)\left(\mathrm{ft}^{3}\right.\end{array}$ & $\frac{10}{3 / s)} \mathrm{Ac}$ & 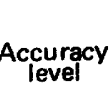 \\
\hline UW53 & Rib River & $\begin{array}{l}\text { NE } 1 / 4 S W 1 / 4 \text { sec. } 6, \text { T. } 28 \text { N., R. } 6 \text { E., } \\
\text { Marathon County, at bridge on } \\
\text { State Highway 107, at Marathon, } \\
\text { Wis. }\end{array}$ & 365 & $\begin{array}{l}\text { Oct. } 24,1968 \\
\text { Aug. } 27,1969 \\
\text { June } 22,1973 \\
\text { Aug. } 15,1973 \\
\text { Oct. } 2,1973 \\
\text { July } 8,1976\end{array}$ & $\begin{array}{c}96.4 \\
27.5 \\
109 \\
55.2 \\
92.6 \\
21.2\end{array}$ & 29 & 17 & $c$ \\
\hline UW55 & Black Creek & $\begin{array}{l}\text { SW1/4SE1/4 sec. } 31, \text { T. } 30 \text { N., R. } 4 \text { E., } \\
\text { Marathon County, at bridge on } \\
\text { State Highway 97, at Athens, Wis. }\end{array}$ & 39.9 & 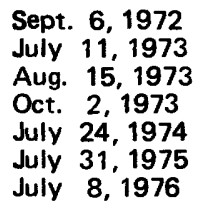 & $\begin{array}{l}6.02 \\
2.77 \\
2.82 \\
1.50 \\
1.75 \\
.40 \\
.58\end{array}$ & .72 & .19 & c \\
\hline \multirow[t]{2}{*}{ UW58 } & Scotch Creek & $\begin{array}{l}\text { SE } 1 / 4 S W 1 / 4 \text { sec. } 7, T .28 \text { N., R. } 5 \text { E., } \\
\text { Marathon County, on town road, } \\
0.1 \text { mi east of County Trunk H, } \\
\text { in Edgar, Wis. }\end{array}$ & 15.3 & & & & & \\
\hline & Scotch Creek & $\begin{array}{l}\text { SE } 1 / 4 \text { SE } 1 / 4 \text { sec. } 7, \text { T. } 28 \text { N., R. } 5 \text { E., } \\
\text { Marathon County, at bridge on } \\
\text { country road, } 0.7 \text { mi east of } \\
\text { County Trunk H in Edgar, Wis. }\end{array}$ & 16.9 & $\begin{array}{l}\text { Sept. } 6,1972 \\
\text { July } 11,1973 \\
\text { Aug. } 15,1973 \\
\text { Oct. } 2,1973 \\
\text { July } 24,1974 \\
\text { July } 31,1975 \\
\text { July } 8,1976\end{array}$ & $\begin{array}{l}3.65 \\
1.03 \\
.46 \\
.64 \\
.42 \\
.13 \\
.13\end{array}$ & .23 & .04 & c \\
\hline Uw67 & Spring Brook & $\begin{array}{l}\text { SE } 1 / 1 N^{\prime} 1 / / \text { sec. } 4, T .30 \text { N., R. } 11 \text { E., } \\
\text { Langlade County, upstream from } \\
\text { sewage-treatment plant, } 2.1 \mathrm{mi} \\
\text { southwest of Antigo, Wis. }\end{array}$ & 39.6 & $\begin{array}{l}\text { July } 10,1972 \\
\text { June } 19,1973 \\
\text { Aug. } 28,1973 \\
\text { July } 15,1975 \\
\text { Aug. } 2,1975 \\
\text { Sept. 14, } 1976 \\
\text { Aug. } 16,1977\end{array}$ & $\begin{array}{l}10.4 \\
9.68 \\
33.8 \\
6.77 \\
19.8 \\
7.44 \\
3.84\end{array}$ & 4.6 & 2.5 & $c$ \\
\hline \multirow[t]{2}{*}{ UW72 } & $\begin{array}{l}\text { West Branch } \\
\text { Big Eau Pleine } \\
\text { River }\end{array}$ & $\begin{array}{l}\text { NE } 1 / 4 S^{\prime} 1 / 4 \text { sec. } 19, T .30 \text { N., R. } 2 \text { E., } \\
\text { Taylor County, at bridge on town } \\
\text { road, } 0.9 \text { mi southeast of } \\
\text { Stetsonville, Wis. }\end{array}$ & 2.95 & & & & & \\
\hline & $\begin{array}{l}\text { West Branch } \\
\text { Big Eau Pleine } \\
\text { River }\end{array}$ & $\begin{array}{l}\text { SW1/sW1/4 sec. } 20, T .30 \text { N., R. } 2 \text { E., } \\
\text { Taylor County, at bridge on country } \\
\text { road, } 1.3 \text { mi southeast of } \\
\text { Stetsonville, Wis. }\end{array}$ & 3.31 & 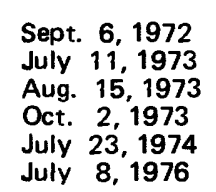 & $\begin{array}{l}{ }^{4} 0 \\
2_{0}^{0} \\
2_{0}^{.01} \\
2_{0}^{.01}\end{array}$ & 0 & 0 & c \\
\hline UW75 & Randall Creek & $\begin{array}{l}\text { SE } 1 / 2 N E 1 / 4 \text { sec. } 21, T .29 \text { N., R. } 3 \text { E., } \\
\text { Marathon County, at bridge on } \\
\text { country road, } 1.9 \text { mi east of } \\
\text { Milan, Wis. }\end{array}$ & 3.93 & $\begin{array}{l}\text { July } 8,1976 \\
\text { Sept. } 13,1976 \\
\text { Aug. } 15,1977\end{array}$ & $\begin{array}{l}20 \\
20 \\
40\end{array}$ & 0 & 0 & c \\
\hline UW76 & Dill Creek & $\begin{array}{l}\text { NW1/aSW1// sec. } 13, T .28 \text { N., R. } 1 \text { E., } \\
\text { Clark County, at bridge on County } \\
\text { Trunk N, } 0.9 \text { mi west of State } \\
\text { Highway } 13 \text { in Colby, Wis. }\end{array}$ & 12.3 & $\begin{array}{l}\text { Sept. } 7,1972 \\
\text { July } 11,1973 \\
\text { Aug. } 15,1973 \\
\text { Oct. } 2,1973 \\
\text { July } 24,1974 \\
\text { July } 8,1976 \\
\text { Sept. } 13,1976 \\
\text { Aug. } 15,1977\end{array}$ & $\begin{array}{l}{ }^{4} 0 \\
.32 \\
.32 \\
.78 \\
.18 \\
.19 \\
.15 \\
.36\end{array}$ & .19 & .09 & 9 \\
\hline UW77 & Elm Brook & $\begin{array}{l}\text { SE } 1 / 4 S W 1 / 4 \text { sec. } 31, \text { T. } 29 \text { N., R. } 2 \text { E., } \\
\text { Marathon County, at bridge on State } \\
\text { Highway } 29 \text {, in Abbotsford, Wis. }\end{array}$ & .31 & $\begin{array}{l}\text { Sept. } 7,1972 \\
\text { July } 11,1973 \\
\text { Aug. } 15,1973 \\
\text { Oct. } 2,1973 \\
\text { July } 8,1976\end{array}$ & $\begin{array}{l}.18 \\
.03 \\
<.03 \text { est. } \\
.06 \\
<.02 \text { est. }\end{array}$ & t. & $<.01$ & 1 \\
\hline UW85 & $\begin{array}{l}\text { Big Eau } \\
\text { Pleine River }\end{array}$ & $\begin{array}{l}\text { NWY/4WY } 1 / 4 \text { sec. } 18, \text { T. } 27 \text { N., R. } 4 \text { E., } \\
\text { Marathon County, at bridge on } \\
\text { State Highway } 97,1.2 \text { mi north of } \\
\text { Stratford, Wis. }\end{array}$ & 224 & & & 2.2 & .50 & 50 \\
\hline
\end{tabular}


Table 29. Upper Wisconsin River basin station locations and low-flow characteristics-Continued.

\begin{tabular}{|c|c|c|c|c|c|c|c|c|}
\hline $\begin{array}{l}\text { Station } \\
\text { number }\end{array}$ & ${ }^{1} \underset{\text { Stream }}{\text { name }}$ & Station location & $\begin{array}{l}\text { Drainage } \\
\text { area } \\
\left(\mathrm{mi}^{2}\right)\end{array}$ & Date & $\begin{array}{c}\text { Discharge } \\
\left(\mathrm{ft}^{3} / \mathrm{s}\right)\end{array}$ & $\begin{array}{l}\mathrm{Q}_{7,2} \mathrm{O}_{7} \\
\left(\mathrm{ft}^{3} / \mathrm{s}\right)(\mathrm{ft})\end{array}$ & $\left.t^{3} / s\right)$ & $\underset{\substack{\text { Aevel } \\
\text { levacy }}}{\text { Actas }}$ \\
\hline UW88 & Peplin Creek & $\begin{array}{l}\text { SE } 1 / 4 \text { SW } 1 / 4 \text { sec. } 35, T .27 \text { N., R. } 7 \text { E., } \\
\text { Marathon County, at bridge on } \\
\text { country road, } 3.3 \text { mi southeast } \\
\text { of Mosinee, Wis. }\end{array}$ & 6.37 & $\begin{array}{l}\text { Julý } 11,1972 \\
\text { Sept. } 6,1972 \\
\text { June } 22,1973 \\
\text { Aug. } 15,1973 \\
\text { Oct. } 2,1973 \\
\text { July } 23,1974 \\
\text { July } 8,1976\end{array}$ & $\begin{array}{l}{ }^{4}{ }_{0}^{0} \\
.02 \\
4_{0}^{.02} \\
4_{0}^{0} \\
4_{0}^{0}\end{array}$ & 0 & 0 & c \\
\hline UW89 & $\begin{array}{l}\text { Little Eau } \\
\text { Pleine River }\end{array}$ & $\begin{array}{l}\text { SW1/4NE } 1 / 4 \text { sec. } 1, \text { T. } 27 \text { N., R. } 1 \text { E., } \\
\text { Clark County, at sewage-disposal } \\
\text { lagoon, } 0.5 \text { mi northwest of } \\
\text { Unity, Wis. }\end{array}$ & 7.42 & $\begin{array}{l}\text { Sept. } 7,1972 \\
\text { July } 11,1973 \\
\text { Aug. } 15,1973 \\
\text { Oct. } 2,1973 \\
\text { July } 23,1974 \\
\text { July } 8,1976\end{array}$ & $\begin{array}{l}{ }_{4}^{4} 0 \\
4_{0}^{0} \\
<{ }_{4}^{.01} \text { est. } \\
4_{0}^{0}\end{array}$ & t. & 0 & c \\
\hline UW91 & $\begin{array}{l}\text { Tributary to } \\
\text { Little Eau } \\
\text { Pleine River } \\
\text { tributary }\end{array}$ & $\begin{array}{l}\text { SW } 1 / 4 \text { SE } 1 / 4 \text { sec. } 5, \text { T. } 26 \text { N., R. } 2 \text { E., } \\
\text { Marathon County, at bridge on } \\
\text { County Trunk C, } 0.6 \text { mi east of } \\
\text { Spencer, Wis. }\end{array}$ & 1.05 & $\begin{array}{l}\text { Sept. } 7,1972 \\
\text { July } 11,1973 \\
\text { Aug. } 15,1973 \\
\text { Oct. } 2,1973 \\
\text { July } 23,1974 \\
\text { July } 8,1976\end{array}$ & $\begin{array}{l}.21 \\
.03 \text { est. } \\
.02 \text { est. } \\
{ }^{4.01} 0 \\
<0 \\
<.01 \text { est. }\end{array}$ & $<.01$ & $<.01$ & c \\
\hline UW98 & $\begin{array}{l}\text { Little Bear } \\
\text { Creek }\end{array}$ & $\begin{array}{l}\text { NE } 1 / 4 \text { SE } 1 / 4 \text { sec. } 23, \text { T. } 25 \text { N., R. } 4 \text { E., } \\
\text { Wood County, at sewage-disposal } \\
\text { ponds, } 1.0 \text { mi northeast of } \\
\text { Auburndale, Wis. }\end{array}$ & 1.50 & $\begin{array}{l}\text { July } 11,1972 \\
\text { Sept. } 5,1972 \\
\text { July } 10,1973 \\
\text { Aug. } 14,1973 \\
\text { Oct. } 1,1973 \\
\text { July } 23,1974 \\
\text { July } 7,1976\end{array}$ & $\begin{array}{l}40 \\
4_{0}^{0} \\
4_{0}^{0} \\
{ }_{4}^{0} \\
4_{0}^{0} \\
{ }^{0}\end{array}$ & 0 & 0 & c \\
\hline UW99 & Plover River & $\begin{array}{l}\text { SW1/4NW1/4 sec. } 31, \text { T. } 30 \mathrm{~N} ., \text { R. } 11 \text { E., } \\
\text { Langlede County, at culvert on } \\
\text { County Trunk HH, } 2.1 \text { mi north of } \\
\text { Aniwa, Wis. }\end{array}$ & 6.86 & $\begin{array}{l}\text { July } 10,1972 \\
\text { June } 19,1973 \\
\text { Aug. } 28,1973 \\
\text { July } 15,1975 \\
\text { Aug. } 2,1975 \\
\text { Sept. } 14,1976 \\
\text { Aug. } 16,1977\end{array}$ & $\begin{array}{l}.44 \\
1.80 \\
.73 \\
.08 \\
.27 \\
4.06 \\
0\end{array}$ & .02 & $<.01$ & c \\
\hline UW100I & Mill Creek & $\begin{array}{l}\text { NE } 1 / 4 S E 1 / 4 \text { sec. } 18, \text { T. } 25 \text { N., R. } 3 \text { E., } \\
\text { Wood County, at culvert on town } \\
\text { road, } 1.5 \text { mi south of post office, } \\
\text { in Marshfield, Wis. }\end{array}$ & 2.03 & $\begin{array}{l}\text { July } 11,1972 \\
\text { Sept. } 7,1972 \\
\text { July } 10,1973 \\
\text { Aug. } 14,1973 \\
\text { Oct. } 1,1973 \\
\text { July } 24,1974 \\
\text { July } 7,1976 \\
\text { Sept. } 13,1976 \\
\text { Aug. } 15,1977\end{array}$ & $\begin{array}{l}.45 \\
.38 \\
.61 \\
.62 \\
.10 \\
.22 \\
.29 \\
.21 \\
.28\end{array}$ & $\underline{7}$ & 7 & \\
\hline & Mill Creek & $\begin{array}{l}\text { SE } 1 / 4 \text { SW } 1 / 4 \text { sec. } 17, \text { T. } 25 \text { N., R. } 3 \text { E., } \\
\text { Wood County, at sewage-treatment } \\
\text { plant, } 1.7 \text { mi south of post office } \\
\text { in Marshfield, Wis. }\end{array}$ & 2.44 & & & & & \\
\hline UW102I & $\begin{array}{l}\text { Mill Creek } \\
\text { tributary }\end{array}$ & $\begin{array}{l}\text { NW1/4NEY/4 sec. 12, T. } 24 \text { N., R. } 5 \text { E., } \\
\text { Wood County, at bridge on country } \\
\text { road, } 1.5 \text { mi south of Milladore, } \\
\text { Wis. }\end{array}$ & 2.63 & 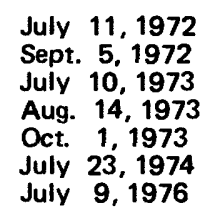 & $\begin{array}{l}20 \\
20 \\
2 \\
2 \\
2 \\
2 \\
4 \\
4 \\
4\end{array}$ & 0 & 0 & c \\
\hline UW103 I & $\begin{array}{l}\text { Mill Creek } \\
\text { tributary }\end{array}$ & $\begin{array}{l}\text { NE } 1 / 4 N W 1 / 4 \text { sec. } 11, \text { T. } 24 \text { N., R. } 6 \text { E., } \\
\text { Portage County, at sewage-disposal } \\
\text { ponds, } 0.5 \text { mi south of Junction } \\
\text { City, Wis. }\end{array}$ & 1.40 & July 10, 1972 & ${ }^{2} 0$ & 0 & 0 & c \\
\hline UW105 t & $\begin{array}{l}\text { Wisconsin River } \\
\text { tributary }\end{array}$ & $\begin{array}{l}\text { SE } 1 / 1 / 2 W 1 / 4 \text { sec. 9, T. } 23 \text { N., R. } 6 \text { E., } \\
\text { Wood County, at sewage-disposal } \\
\text { pond, at Rudolph, Wis. }\end{array}$ & .31 & $\begin{array}{l}\text { Sept. } 5,1972 \\
\text { July } 10,1973 \\
\text { Oct. } 1,1973 \\
\text { July } 23,1974 \\
\text { July } 9,1976\end{array}$ & $\begin{array}{l}20 \\
2_{0}^{0} \\
20 \\
20 \\
20\end{array}$ & $\mathbf{0}$ & 0 & c \\
\hline
\end{tabular}


Table 29. Upper Wisconsin River basin station locations and low-flow characteristics-Continued.

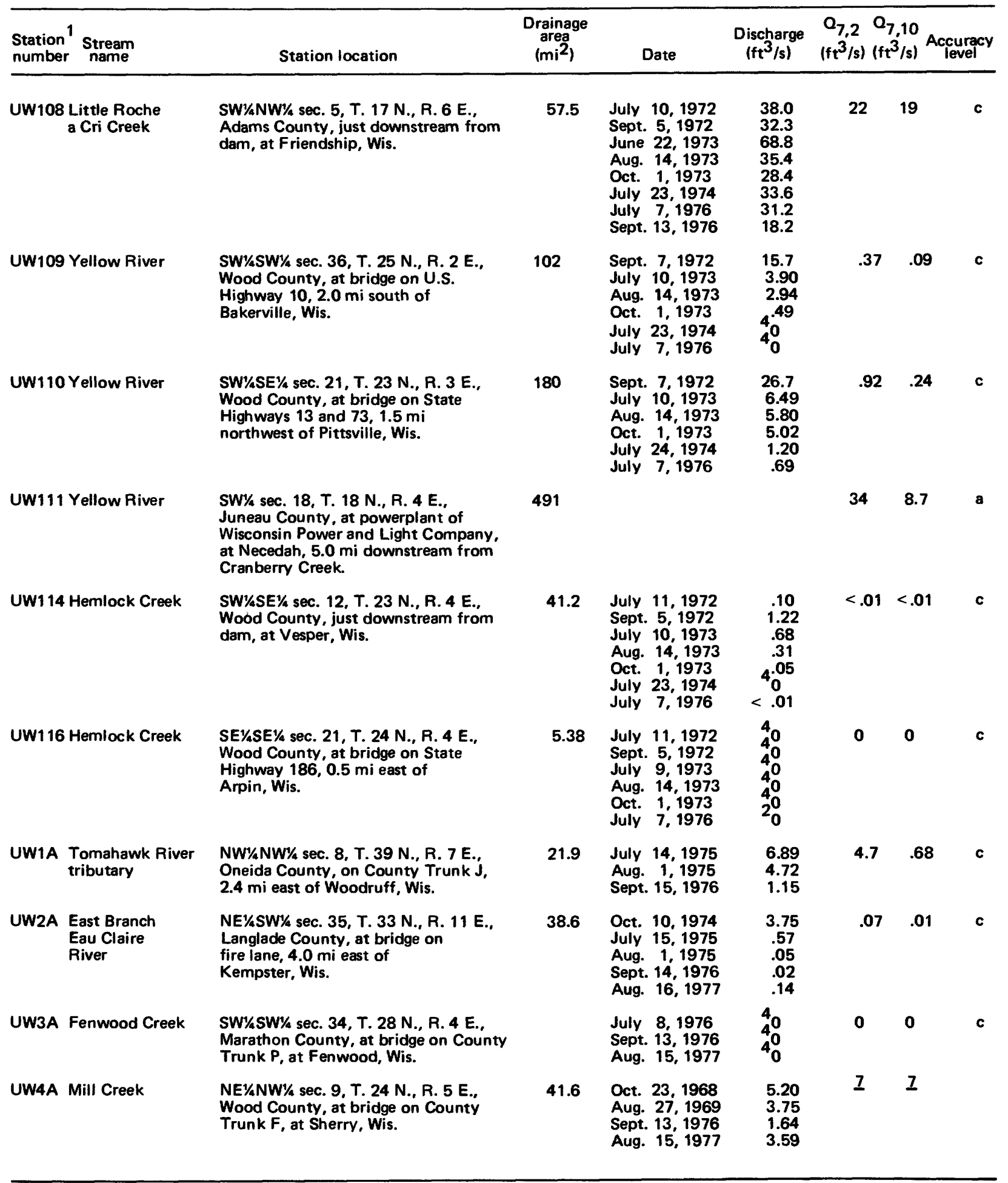

\footnotetext{
1 Based on 1970 report by Wisconsin Department of Natural Resources (DNR). Site lettered "A" was not included in 1970 DNR report.

2 Streambed is dry.

${ }^{4}$ Negligible discharge-water ponded, unable to measure velocity.

${ }^{6}$ No estimate possible due to regulation upstream.

${ }^{7}$ Unable to define relationship.
} 
Table 30. Wolf River basin station locations and low-flow characteristics.

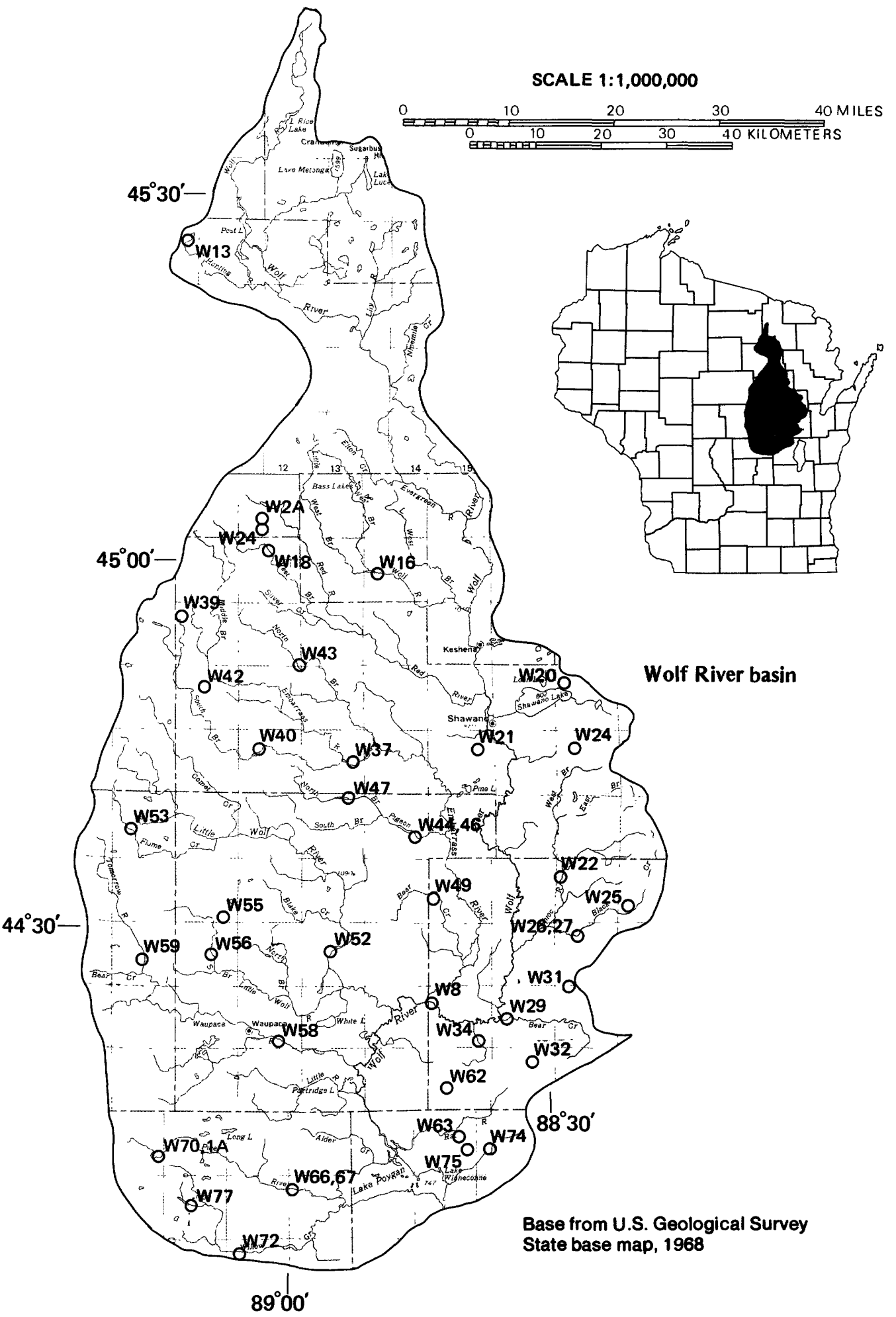

\begin{tabular}{|c|c|c|c|c|c|c|}
\hline $\begin{array}{l}\text { Station } \\
\text { number }\end{array}$ & Station location & $\begin{array}{l}\text { Drainage } \\
\text { area } \\
\left(\mathrm{mi}^{2}\right)\end{array}$ & Date & $\begin{array}{c}\text { Discharge } \\
\left(\mathrm{ft}^{3} / \mathrm{s}\right)\end{array}$ & $\begin{array}{l}Q_{7,2} Q_{7,10} \\
\left(\mathrm{ft}^{3} / \mathrm{s}\right)\left(\mathrm{ft}^{3} / \mathrm{s}\right)\end{array}$ & $\begin{array}{c}\text { Accuracy } \\
\text { level }\end{array}$ \\
\hline Wolf River & $\begin{array}{l}\text { SE1/4 sec. 12, T. } 22 \text { N., R. } 14 \text { E., } \\
\text { Waupaca County, at Pearl Street } \\
\text { Bridge, at New London, Wis. }\end{array}$ & 2,240 & & & 670 & $\mathbf{a}$ \\
\hline
\end{tabular}


Table 30. Wolf River basin station locations and low-flow characteristics-Continued.

\begin{tabular}{|c|c|c|c|c|c|c|c|c|}
\hline $\begin{array}{l}\text { Statior } \\
\text { numbe }\end{array}$ & $n^{1}$ Stream & Station location & $\begin{array}{l}\text { Drainage } \\
\text { area } \\
\left(\mathrm{mi}^{2}\right)\end{array}$ & Date & $\begin{array}{c}\text { Discharge } \\
\left(\mathrm{ft}^{3} / \mathrm{s}\right)\end{array}$ & $\begin{array}{l}\mathrm{Q}_{7,2} \mathrm{Q}_{7} \\
\left(\mathrm{ft}^{3} / \mathrm{s}\right)\left(\mathrm{ft}^{3}\right.\end{array}$ & $\begin{array}{l}7,10 \\
3 / s)\end{array}$ & $\begin{array}{l}\text { Accuracy } \\
\text { level }\end{array}$ \\
\hline w13 & Hunting River & $\begin{array}{l}\mathrm{SE} 1 / 4 \mathrm{SE}^{1 / 4} \text { sec. } 11, \mathrm{~T} .34 \mathrm{~N} ., \mathrm{R} .10 \mathrm{E} ., \\
\text { Langlade County, at culvert on } \\
\text { country road, } 0.9 \text { mi west of } \\
\text { Elcho, Wis. }\end{array}$ & 1.69 & $\begin{array}{l}\text { Aug. } 2,1972 \\
\text { June } 19,1973 \\
\text { July } 15,1973 \\
\text { July } 15,1975 \\
\text { Aug. } 1,1975 \\
\text { Sept. 14, } 1976\end{array}$ & $\begin{array}{l}0.51 \\
2.31 \\
5.82 \\
.67 \\
.41 \\
.73\end{array}$ & 0.41 & 0.26 & c \\
\hline W16 & $\begin{array}{l}\text { West Branch } \\
\text { Wolf River }\end{array}$ & $\begin{array}{l}\text { SEY } 1 / 4 W 1 / 4 \text { sec. } 20, \text { T. } 29 \text { N., R. } 14 \text { E., } \\
\text { Menomonee County, at dam and } \\
\text { powerplant, at Neopit, Wis. }\end{array}$ & 108 & $\begin{array}{lr}\text { Aug. } & 2,1972 \\
\text { July } 10,1973 \\
\text { Aug. } 14,1973 \\
\text { June } 26,1974 \\
\text { July } 23,1974 \\
\text { July } 15,1975\end{array}$ & $\begin{array}{c}3113 \\
143 \\
122 \\
85.1 \\
87.6 \\
107\end{array}$ & 65 & 32 & b \\
\hline W18 & $\begin{array}{l}\text { West Branch } \\
\text { Red River }\end{array}$ & $\begin{array}{l}\text { NE } 1 / 4 \text { NE } 1 / 4 \text { sec. } 9, T .29 \text { N., R. } 12 \text { E., } \\
\text { Shawano County, at County Trunk D, } \\
0.3 \text { mi north of Mattoon, Wis. }\end{array}$ & 21.9 & $\begin{array}{lr}\text { Oct. } & 19,1965 \\
\text { Aug. } & 2,1972 \\
\text { July } & 11,1973 \\
\text { Aug. } & 14,1973 \\
\text { July } & 23,1974 \\
\text { July } & 15,1975 \\
\text { July } & 8,1976\end{array}$ & $\begin{array}{l}23.2 \\
14.6 \\
19.4 \\
11.3 \\
10.2 \\
10.9 \\
12.2\end{array}$ & 8.4 & 5.6 & c \\
\hline W20 & Duchess Creek & $\begin{array}{l}\text { SW } 1 / 4 \text { NW } 1 / 4 \text { sec. } 8, \text { T. } 27 \text { N., R. } 17 \text { E., } \\
\text { Shawano County, at culvert on } \\
\text { County Trunk H, } 1.8 \text { mi northwest } \\
\text { of Cecil, Wis. }\end{array}$ & 6.58 & $\begin{array}{l}\text { Aug. } 2,1972 \\
\text { July } 10,1973 \\
\text { Aug. } 14,1973 \\
\text { July } 24,1974 \\
\text { July } 16,1975 \\
\text { July } 8,1976 \\
\text { July } 27,1977\end{array}$ & $\begin{array}{l}1.20 \\
1.46 \\
.99 \\
.10 \\
1.36 \\
1.26 \\
.05\end{array}$ & .05 & .01 & c \\
\hline W21 & $\begin{array}{l}\text { Wolf River } \\
\text { tributary }\end{array}$ & $\begin{array}{l}\text { SW } 1 / 4 \text { NW } 1 / 4 \text { sec. } 13, T .26 \mathrm{~N} ., \mathrm{R} .15 \mathrm{E} . \text {., } \\
\text { Shawano County, at bridge on country } \\
\text { road, } 1.2 \text { mi southeast of Shawano } \\
\text { County Hospital, } 2.3 \text { mi northeast of } \\
\text { Belle Plaine, Wis. }\end{array}$ & 6.22 & $\begin{array}{l}\text { Aug. } 1,1972 \\
\text { July } 11,1973 \\
\text { Aug. } 15,1973 \\
\text { July } 24,1974 \\
\text { July } 14,1975 \\
\text { July } 7,1976\end{array}$ & $\begin{array}{l}2 \\
20 \\
20 \\
20 \\
2_{0}^{0} \\
2_{0}^{0}\end{array}$ & 0 & $\mathbf{0}$ & c \\
\hline W22 & Shioc River & $\begin{array}{l}\text { NE } 1 / 4 \text { SW } 1 / 4 \text { sec. } 7, T .24 \text { N., R. } 17 \text { E., } \\
\text { Outagamie County, at State Highway } \\
156,0.8 \text { mi west of Nichols, Wis. }\end{array}$ & 92.0 & $\begin{array}{l}\text { Aug. } 1,1972 \\
\text { July } 10,1973 \\
\text { Aug. } 14,1973 \\
\text { July } 24,1974 \\
\text { July } 14,1975 \\
\text { July } 6,1976 \\
\text { July } 27,1977\end{array}$ & $\begin{array}{c}32.62 \\
7.66 \\
5.57 \\
11.1 \\
14.9 \\
2.16 \\
.09\end{array}$ & .33 & .06 & b \\
\hline W24 & $\begin{array}{l}\text { West Branch } \\
\text { Shioc River }\end{array}$ & $\begin{array}{l}\text { SW } 1 / 4 S^{1} 1 / 4 \text { sec. } 8, \text { T. } 26 \mathrm{~N} ., \text { R. } 17 \mathrm{E} . \text {, } \\
\text { Shawano County, } 0.2 \mathrm{mi} \text { upstream } \\
\text { from country road, } 0.3 \mathrm{mi} \text { southwest } \\
\text { of Bonduel, Wis. }\end{array}$ & 4.00 & $\begin{array}{lc}\text { Aug. } & 2,1972 \\
\text { July } & 10,1973 \\
\text { Aug. } 14,1973 \\
\text { July } 24,1974 \\
\text { July } 16,1975 \\
\text { July } 8,1976\end{array}$ & $\begin{array}{l}.45 \\
1.53 \\
1.60 \\
.31 \\
.58 \\
.36\end{array}$ & .06 & .02 & c \\
\hline W25 & Black Creek & $\begin{array}{l}\text { SE } 1 / 4 \text { SW } 1 / 4 \text { sec. } 29, \text { T. } 24 \text { N., R. } 18 \text { E., } \\
\text { Outagamie County, at bridge on } \\
\text { County Trunk G, } 0.8 \text { mi west of } \\
\text { Seymour, Wis. }\end{array}$ & 24.1 & $\begin{array}{l}\text { Aug. } 13,1969 \\
\text { Aug. } 29,1969 \\
\text { Aug. } 1,1972 \\
\text { July } 10,1973 \\
\text { Aug. } 14,1973 \\
\text { July } 24,1974 \\
\text { July } 14,1975 \\
\text { July } 6,1976\end{array}$ & $\begin{array}{l}.16 \\
.09 \\
.15 \\
.27 \\
.19 \\
.16 \\
.73 \\
.15\end{array}$ & .07 & .04 & c \\
\hline $\begin{array}{l}\text { W26, } \\
27\end{array}$ & Black Creek & $\begin{array}{l}\text { SE } 1 / 4 N^{1} / 4 \text { sec. } 8, T .23 \text { N., R. } 17 \text { E., } \\
\text { Outagamie County, at State Highway } \\
47,0.3 \text { mi north of Black Creek, } \\
\text { Wis. }\end{array}$ & 55.9 & $\begin{array}{l}\text { Aug. } 1,1972 \\
\text { July } 10,1973 \\
\text { Aug. } 14,1973 \\
\text { July } 24,1974 \\
\text { July } 14,1975\end{array}$ & $\begin{array}{c}3.11 \\
2.78 \\
.70 \\
1.36 \\
<.10 \text { est. }\end{array}$ & .10 & .03 & b \\
\hline W29 & Bear Creek & $\begin{array}{l}\text { NW1/aNE1/4 sec. } 20, \text { T.2 } 2 \text { N., R. } 16 \text { E., } \\
\text { Outagamie County, at State Highway } \\
\text { 76, at Stephensville, Wis. }\end{array}$ & 59.4 & $\begin{array}{l}\text { July } 31,1972 \\
\text { July } 10,1973 \\
\text { Aug. } 13,1973 \\
\text { July } 14,1975 \\
\text { July } 8,1976\end{array}$ & $\begin{array}{l}3.92 \\
6.55 \\
2.33 \\
3.52 \\
.81\end{array}$ & .54 & .23 & b \\
\hline
\end{tabular}


Table 30. Wolf River basin station locations and low-flow characteristics-Continued.

\begin{tabular}{|c|c|c|c|c|c|c|c|c|}
\hline $\begin{array}{l}\text { Station } \\
\text { numbel }\end{array}$ & $\mathrm{n}^{1} \underset{\text { er }}{\text { Streame }}$ & Station location & $\begin{array}{l}\text { Drainage } \\
\text { area } \\
\left(\mathrm{mi}^{2}\right)\end{array}$ & Date & $\begin{array}{l}\text { Discharge } \\
\left(\mathrm{ft}^{3} / \mathrm{s}\right)\end{array}$ & $\begin{array}{l}\mathrm{Q}_{7,2} \mathrm{Q}_{7} \\
\left(\mathrm{ft}^{3} / \mathrm{s}\right)(\mathrm{ft}\end{array}$ & $\begin{array}{l}7,10 \\
\left.t^{3} / \mathrm{s}\right)\end{array}$ & $\begin{array}{c}\text { Accuracy } \\
\text { level }\end{array}$ \\
\hline W31 & $\begin{array}{l}\text { Tributary to } \\
\text { Bear Creek } \\
\text { tributary }\end{array}$ & $\begin{array}{l}\text { SW } 1 / 4 \text { SE } 1 / 4 \text { sec. } 5, \text { T. } 22 \text { N., R. } 17 \text { E., } \\
\text { Outagamie County, at bridge on } \\
\text { country road, } 5.2 \text { mi south of } \\
\text { Black Creek, Wis. }\end{array}$ & 2.19 & $\begin{array}{l}\text { Aug. } 1,1972 \\
\text { July } 10,1973 \\
\text { Aug. } 14,1973 \\
\text { July } 14,1975 \\
\text { July } 8,1976\end{array}$ & $\begin{array}{l}4_{0}^{0} \\
4_{0}^{0} \\
4_{0}^{0} \\
4_{0}^{0}\end{array}$ & 0 & 0 & c \\
\hline W32 & Bear Creek & $\begin{array}{l}\text { NW1/4NW1/4 sec. } 14, \text { T. } 21 \text { N., R. } 16 \text { E., } \\
\text { Outagamie County, at culvert on } \\
\text { State Highway } 76 \text {, at Greenville, } \\
\text { Wis. }\end{array}$ & 1.98 & $\begin{array}{l}\text { July } 31,1972 \\
\text { July } 9,1973 \\
\text { Aug. } 13,1973 \\
\text { July } 14,1975 \\
\text { July } 8,1976\end{array}$ & $\begin{array}{l}2 \\
20 \\
20 \\
2_{0}^{0} \\
{ }_{4}^{0} \\
0\end{array}$ & 0 & 0 & c \\
\hline W34 & $\begin{array}{l}\text { Black Otter } \\
\text { Creek }\end{array}$ & $\begin{array}{l}\text { NW1/4SE1/4 sec. } 35, T .22 \text { N., R. } 15 \text { E., } \\
\text { Outagamie County, at local road } \\
0.2 \text { mi north of U.S. Highway } 45 \text {, } \\
\text { northwest edge of Hortonville, Wis. }\end{array}$ & 15.9 & $\begin{array}{l}\text { Aug. } 12,1969 \\
\text { Aug. } 27,1969 \\
\text { July } 31,1972 \\
\text { July } 9,1973 \\
\text { Aug. } 14,1973 \\
\text { July } 14,1975 \\
\text { July } 8,1976\end{array}$ & $\begin{array}{c}1.25 \\
.48 \\
.84 \\
1.75 \\
.82 \\
1.19 \\
.78\end{array}$ & .47 & .29 & c \\
\hline \multirow[t]{2}{*}{ W37 } & Embarrass River & $\begin{array}{l}\text { SW1/4NW1/4 sec. } 24, \text { T. } 26 \text { N., R. } 13 \text { E., } \\
\text { Shawano County, at sewage lagoon, } \\
0.7 \text { mi east of Caroline, Wis. }\end{array}$ & 264 & & & & & \\
\hline & Embarrass River & $\begin{array}{l}\text { SWYSW1/4 sec. 13, T. } 26 \text { N., R. } 13 \text { E., } \\
\text { Shawano County, at town road, } \\
0.9 \text { mi east of Caroline, Wis. }\end{array}$ & 265 & $\begin{array}{l}\text { Aug. } 3,1972 \\
\text { July } 11,1973 \\
\text { Aug. } 15,1973 \\
\text { July } 24,1974 \\
\text { July } 7,1976\end{array}$ & $\begin{array}{c}3145 \\
135 \\
114 \\
62.0 \\
42.4\end{array}$ & 46 & 26 & c \\
\hline \multirow[t]{2}{*}{ W39 } & Railroad Creek & $\begin{array}{l}\text { NEY } 1 / \text { NE } 1 / 4 \text { sec. } 7, \text { T. } 28 \text { N., R. } 11 \text { E., } \\
\text { Shawano County, at sewage-treatment } \\
\text { plant, at Birnamwood, Wis. }\end{array}$ & .52 & & & & & \\
\hline & Railroad Creek & $\begin{array}{l}\text { NE } 1 / \text { SE } / / \text { sec. } 18, T .28 \text { N., R. } 11 \text { E., } \\
\text { Shawano County, at culvert on } \\
\text { country road, } 1.7 \text { mi south of } \\
\text { Birnamwood, Wis. }\end{array}$ & 3.17 & $\begin{array}{l}\text { Aug. } 2,1972 \\
\text { July } 11,1973 \\
\text { Aug. } 15,1973 \\
\text { July } 15,1975 \\
\text { July } 8,1976\end{array}$ & $\begin{array}{l}1.44 \\
1.57 \\
1.07 \\
1.21 \\
.95\end{array}$ & .64 & .39 & c \\
\hline W40 & $\begin{array}{l}\text { South Branch } \\
\text { Embarrass River }\end{array}$ & $\begin{array}{l}\text { NE } 1 / 4 \text { SW } 1 / 4 \text { sec. } 9, \text { T. } 26 \text { N., R. } 12 \text { E., } \\
\text { Shawano County, at U.S. Highway } \\
45 \text { bridge, at Tigerton, Wis. }\end{array}$ & 94.8 & $\begin{array}{l}\text { Aug. } 3,1972 \\
\text { July } 11,1973 \\
\text { Aug. } 15,1973 \\
\text { July } 24,1974 \\
\text { July } 15,1975 \\
\text { July } 7,1976\end{array}$ & $\begin{array}{l}52.1 \\
56.8 \\
77.7 \\
34.9 \\
38.9 \\
25.1\end{array}$ & 23 & 14 & c \\
\hline W42 & Tiger Creek & $\begin{array}{l}\text { SW1/4NW1/4 sec. 15, T. } 27 \text { N., R. } 11 \text { E., } \\
\text { Shawano County, at sewage-treatment } \\
\text { plant, at Wittenberg, Wis. }\end{array}$ & 1.08 & $\begin{array}{l}\text { Aug. } 3,1972 \\
\text { July } 11,1973 \\
\text { Aug. } 15,1973 \\
\text { July } 24,1974 \\
\text { July } 15,1975 \\
\text { July } 7,1976\end{array}$ & $\begin{array}{l}.82 \\
.59 \\
.79 \\
.59 \\
.63 \\
.20\end{array}$ & $\underline{5}$ & $\underline{5}$ & \\
\hline W43 & $\begin{array}{l}\text { North Branch } \\
\text { Embarrass River }\end{array}$ & $\begin{array}{l}\text { NW1/4NW1/4 sec. } 6 . \text { T. } 27 \text { N., R. } 13 \text { E., } \\
\text { Shawano County, at County Trunk D, } \\
0.6 \text { mi south of Bowler, Wis. }\end{array}$ & 39.5 & & & 9.8 & 6.0 & b \\
\hline $\begin{array}{l}\text { W44, } \\
46\end{array}$ & Pigeon River & $\begin{array}{l}\text { SE } 1 / 4 \text { SE } 1 / 4 \text { sec. } 23, T .25 \text { N., R. } 14 \text { E., } \\
\text { Waupaca County, at footbridge, } \\
0.1 \text { mi downstream from Main Street } \\
\text { bridge, at Clintonville, Wis. }\end{array}$ & 109 & $\begin{array}{l}\text { Aug. } 11,1969 \\
\text { Aug. } 28,1969 \\
\text { Aug. } 1,1972 \\
\text { July } 12,1973 \\
\text { Aug. } 15,1973 \\
\text { July } 24,1974 \\
\text { July } 14,1975 \\
\text { July } 7,1976\end{array}$ & $\begin{array}{l}36.3 \\
16.4 \\
30.4 \\
61.9 \\
53.6 \\
34.9 \\
39.2 \\
21.2\end{array}$ & 16 & 8.1 & c \\
\hline W47 & $\begin{array}{l}\text { North Branch } \\
\text { Pigeon Creek }\end{array}$ & $\begin{array}{l}\text { SWYNWW1/4 sec. 1, T. } 25 \text { N., R. } 13 \text { E., } \\
\text { Waupaca County, } 0.4 \text { mi upstream } \\
\text { from bridge on U.S. Highway } 45, \\
\text { at Marion sewage-treatment plant. }\end{array}$ & 20.4 & $\begin{array}{l}\text { July } 12,1973 \\
\text { Aug. } 15,1973 \\
\text { July } 24,1974 \\
\text { July } 15,1975 \\
\text { July } 7,1976\end{array}$ & $\begin{array}{l}12.0 \\
28.2 \\
7.30 \\
8.20 \\
3.69\end{array}$ & 3.3 & 1.7 & c \\
\hline
\end{tabular}


Table 30. Wolf River basin station locations and low-flow characteristics-Continued.

\begin{tabular}{|c|c|c|c|c|c|c|c|c|}
\hline $\begin{array}{l}\text { Station } \\
\text { numbe }\end{array}$ & ${ }^{1} \underset{\text { name }}{\text { Stream }}$ & Station location & $\begin{array}{l}\text { Drainage } \\
\text { area } \\
\left(\mathrm{mi}^{2}\right)\end{array}$ & Date & $\begin{array}{c}\text { Discharge } \\
\left(\mathrm{ft}^{3} / \mathrm{s}\right)\end{array}$ & $\begin{array}{l}\mathrm{Q}_{7,2} \\
\left(\mathrm{ft}^{3} / \mathrm{s}\right)\end{array}$ & $\begin{array}{r}\mathrm{Q}_{7,10} \\
\left(\mathrm{ft} \mathrm{t}^{3} / \mathrm{s}\right)\end{array}$ & $\underset{\text { level }}{\text { Accuracy }}$ \\
\hline W49 & Bear Creek & $\begin{array}{l}\text { SE } 1 / 4 \text { SW } 1 / 4 \text { sec. } 20, \text { T. } 24 \text { N., R. } 15 \text { E., } \\
\text { Outagamie County, at bridge on } \\
\text { State Highway } 76,0.7 \text { mi east } \\
\text { of Bear Creek, Wis. }\end{array}$ & 16.3 & $\begin{array}{l}\text { Aug. } 1,1972 \\
\text { July } 12,1973 \\
\text { Aug. } 15,1973 \\
\text { July } 24,1974 \\
\text { July } 14,1975 \\
\text { July } 7,1976\end{array}$ & $\begin{array}{l}0.27 \\
2.72 \\
.53 \\
.51 \\
.56 \\
.17\end{array}$ & $<0.01$ & $<0.01$ & c \\
\hline W52 & $\begin{array}{l}\text { Little Wolf } \\
\text { River }\end{array}$ & $\begin{array}{l}\text { NE } 1 / 4 S E 1 / 4 \text { sec. } 16, \text { T. } 23 \text { N., R. } 13 \text { E., } \\
\text { Waupaca County, at Lindsay Park, } \\
\text { just downstream from sewage-treatment } \\
\text { plant, at Manawa, Wis. }\end{array}$ & 314 & $\begin{array}{l}\text { Aug. } 3,1972 \\
\text { July } 12,1973 \\
\text { Aug. } 16,1973 \\
\text { July } 25,1974 \\
\text { July } 13,1975 \\
\text { July } 7,1976\end{array}$ & $\begin{array}{c}137 \\
195 \\
182 \\
189 \\
163 \\
74.2\end{array}$ & 54 & 26 & c \\
\hline W53 & Flume Creek & $\begin{array}{l}\text { NW } 1 / 4 \text { SW } 1 / 4 \text { sec. } 21, \text { T. } 25 \text { N., R. } 10 \text { E., } \\
\text { Portage County, at bridge on State } \\
\text { Highway } 66 \text {, at Rosholt, Wis. }\end{array}$ & 16.0 & $\begin{array}{l}\text { Aug. } 4,1972 \\
\text { July } 12,1973 \\
\text { Aug. } 15,1973 \\
\text { July } 13,1975 \\
\text { July } 6,1976\end{array}$ & $\begin{array}{l}4.62 \\
8.35 \\
8.68 \\
6.20 \\
3.56\end{array}$ & 3.1 & 2.3 & c \\
\hline W55 & $\begin{array}{l}\text { South Branch } \\
\text { Little Wolf } \\
\text { River }\end{array}$ & $\begin{array}{l}\text { NW1/4SW1/4 sec. } 35, \text { T. } 24 \text { N., R. } 11 \text { E., } \\
\text { Waupaca County, at Town Line Road } \\
\text { bridge, at lola, Wis. }\end{array}$ & 33.6 & $\begin{array}{l}\text { Aug. } 4,1972 \\
\text { July } 12,1973 \\
\text { Aug. } 15,1973 \\
\text { July } 13,1975 \\
\text { July } 7,1976\end{array}$ & $\begin{array}{l}17.3 \\
17.1 \\
26.8 \\
18.6 \\
11.4\end{array}$ & 10 & 7.6 & c \\
\hline W56 & $\begin{array}{l}\text { South Branch } \\
\text { Little Wolf } \\
\text { River }\end{array}$ & $\begin{array}{l}\text { SW } 1 / 4 \text { SE } 1 / 4 \text { sec. } 35, \text { T. } 24 \text { N., R. } 11 \text { E. } \\
\text { Waupaca County, at County Trunk B, } \\
\text { bridge, at Scandinavia, Wis. }\end{array}$ & 55.7 & $\begin{array}{l}\text { Aug. } 4,1972 \\
\text { July } 12,1973 \\
\text { Aug. } 15,1973 \\
\text { July } 13,1975 \\
\text { July } 7,1976\end{array}$ & $\begin{array}{l}35.0 \\
44.6 \\
45.5 \\
33.7 \\
19.9\end{array}$ & 17 & 12 & c \\
\hline \multirow[t]{2}{*}{ W58 } & Waupaca River & $\begin{array}{l}\text { NW1/4NW1/4 sec. } 1, \text { T. } 21 \text { N., R. } 12 \text { E., } \\
\text { Waupaca County, at town road, } \\
4.7 \text { mi southeast of Waupaca, Wis. }\end{array}$ & 272 & & & 140 & 115 & $\mathbf{a}$ \\
\hline & Waupaca River & $\begin{array}{l}\text { SEY/4NW/4 sec. } 4, \text { T. } 21 \text { N., R. } 13 \text { E., } \\
\text { Waupaca County, at bridge on } \\
\text { State Highway } 110 \text {, at Weyauwega, Wis. }\end{array}$ & 282 & & & & & \\
\hline W59 & Tomorrow River & $\begin{array}{l}\text { SE } 1 / 4 \text { SE } 1 / 4 \text { sec. } 21, \text { T. } 23 \text { N., R. } 10 \text { E., } \\
\text { Portage County, at bridge on } \\
\text { County Trunk V, at Amherst, Wis. }\end{array}$ & 74.2 & $\begin{array}{l}\text { Aug. } 4,1972 \\
\text { July } 12,1973 \\
\text { Aug. } 15,1973 \\
\text { July } 13,1975 \\
\text { July } 6,1976\end{array}$ & $\begin{array}{l}31.4 \\
51.8 \\
54.1 \\
40.4 \\
32.7\end{array}$ & 26 & 20 & c \\
\hline W62 & $\begin{array}{l}\text { Rat River } \\
\text { tributary }\end{array}$ & $\begin{array}{l}\text { SW } 1 / 4 \text { SE } 1 / 4 \text { sec. } 21, \text { T. } 21 \text { N., R. } 15 \text { E., } \\
\text { Ou tagamie County, at culvert on } \\
\text { U.S. Highway } 10 \text {, on west side of } \\
\text { Dale, Wis. }\end{array}$ & 2.17 & $\begin{array}{lc}\text { July } & 31,1972 \\
\text { July } & 9,1973 \\
\text { Aug. } & 14,1973 \\
\text { July } & 13,1975 \\
\text { July } & 8,1976\end{array}$ & $\begin{array}{l}20 \\
20 \\
.03 \\
.30 \\
.20\end{array}$ & 0 & 0 & c \\
\hline W63 & $\begin{array}{l}\text { Rat River } \\
\text { tributary }\end{array}$ & $\begin{array}{l}\text { SW1/4NE1/4 sec. } 14, \text { T. } 20 \text { N., R. } 15 \text { E., } \\
\text { Winnebago County, at culvert on } \\
\text { Loop Road, } 0.9 \text { mi northwest of } \\
\text { Winchester, Wis. }\end{array}$ & 2.34 & $\begin{array}{l}\text { July } 31,1972 \\
\text { July } 9,1973 \\
\text { Aug. } 14,1973 \\
\text { July } 13,1975 \\
\text { July } 8,1976\end{array}$ & $\begin{array}{l}20 \\
2 \\
2 \\
4 \\
2 \\
2 \\
0\end{array}$ & 0 & 0 & c \\
\hline $\begin{array}{l}\text { W66, } \\
67\end{array}$ & Pine River & $\begin{array}{l}\text { SE } 1 / 4 N W 1 / 4 \text { sec. } 7, \text { T. } 19 \text { N., R. } 13 \text { E., } \\
\text { Waushara County, at State Highway } \\
\text { 49, at Poy Sippi, Wis. }\end{array}$ & 86.4 & $\begin{array}{l}\text { Sept. } 12,1972 \\
\text { July } 12,1973 \\
\text { Aug. } 14,1973 \\
\text { July } 13,1975 \\
\text { July } 8,1976\end{array}$ & $\begin{array}{c}71.5 \\
102 \\
83.4 \\
86.2 \\
65.6\end{array}$ & 60 & 52 & c \\
\hline $\begin{array}{l}\text { W70, } \\
1 A\end{array}$ & Humphrey Creek & $\begin{array}{l}\text { NW } 1 / 4 \text { NW } \% \text { sec. } 30, \text { T. } 20 \text { N., R. } 11 \text { E., } \\
\text { Waushara County, at bridge on } \\
\text { County Trunk A, at Wild Rose, Wis. }\end{array}$ & 27.1 & $\begin{array}{l}\text { Sept. } 12,1972 \\
\text { July } 13,1973 \\
\text { Aug. } 15,1973 \\
\text { July } 12,1975 \\
\text { July } 9,1976 \\
\text { Aug. } 15,1978\end{array}$ & $\begin{array}{l}8.20 \\
12.7 \\
14.5 \\
9.59 \\
9.82 \\
7.69\end{array}$ & 6.6 & 5.6 & c \\
\hline
\end{tabular}


Table 30. Wolf River basin station locations and low-flow characteristics-Continued.

\begin{tabular}{|c|c|c|c|c|c|c|c|c|}
\hline $\begin{array}{l}\text { Station } \\
\text { ruumber }\end{array}$ & Stream & Station location & $\begin{array}{l}\text { Drainage } \\
\text { area } \\
\left(\mathrm{mi}^{2}\right)\end{array}$ & Date & $\begin{array}{l}\text { Discharge } \\
\left(\mathrm{ft}^{3} / \mathrm{s}\right)\end{array}$ & $\begin{array}{l}a_{7,2} Q_{7} \\
\left(\mathrm{ft}^{3} / \mathrm{s}\right)\left(\mathrm{ft}^{3}\right.\end{array}$ & & $\underset{\substack{\text { Accuracy } \\
\text { level }}}{\text { and }}$ \\
\hline W72 & Willow Creek & $\begin{array}{l}\text { SW } 1 / 4 N W 1 / 4 \text { sec. } 8, T .18 \mathrm{~N} ., \text { R. } 12 \text { E., } \\
\text { Waushara County, at bridge on } \\
\text { County Trunk E, } 0.4 \text { mi north of } \\
\text { Redgranite, Wis. }\end{array}$ & 71.7 & $\begin{array}{l}\text { Sept. } 12,1972 \\
\text { July } 12,1973 \\
\text { Aug. } 14,1973 \\
\text { July } 12,1975 \\
\text { July } 9,1976\end{array}$ & $\begin{array}{l}50.3 \\
89.5 \\
83.1 \\
57.4 \\
36.8\end{array}$ & 36 & 30 & c \\
\hline W74 & Arrowhead River & $\begin{array}{l}\text { NE1/4SW1/4 sec. } 20, \text { T. } 20 \text { N., R. } 16 \text { E., } \\
\text { Winnebego County, at bridge on } \\
\text { County Trunk T, at Larsen, Wis. }\end{array}$ & 7.64 & $\begin{array}{l}\text { July } 31,1972 \\
\text { July } 9,1973 \\
\text { Aug. } 14,1973 \\
\text { July } 13,1975 \\
\text { July } 8,1976\end{array}$ & $\begin{array}{l}{ }^{4}{ }_{0}^{0} \\
{ }^{4}{ }_{0}^{0} \\
{ }_{0}^{0}\end{array}$ & 0 & 0 & c \\
\hline W75 & $\begin{array}{l}\text { Arrowhead River } \\
\text { tributary }\end{array}$ & $\begin{array}{l}\text { NWYNW1/4 sec. } 23, \text { T. } 20 \text { N., R. } 16 \text { E., } \\
\text { Winnebego County, at bridge on } \\
\text { country road, } 3.0 \text { mi east of } \\
\text { Larsen, Wis. }\end{array}$ & 1.07 & $\begin{array}{l}\text { July } 31,1972 \\
\text { July } 9,1973 \\
\text { Aug. } 14,1973 \\
\text { July } 13,1975 \\
\text { July } 8,1976\end{array}$ & $\begin{array}{l}{ }_{2}^{0} \\
2_{0}^{0} \\
2_{0}^{4} \\
2_{0}^{0}\end{array}$ & 0 & 0 & c \\
\hline W77 & $\begin{array}{l}\text { Rattlesnake } \\
\text { Creek }\end{array}$ & $\begin{array}{l}\text { SE1/4SE1/4 sec. } 16, T .19 \text { N., R. } 11 \text { E., } \\
\text { Waushara County, below Lake Morris } \\
\text { dam, at Mount Morris, Wis. }\end{array}$ & 20.4 & $\begin{array}{l}\text { Sept. } 12,1972 \\
\text { July } 13,1973 \\
\text { Aug. } 16,1973 \\
\text { July } 12,1975 \\
\text { July } 9,1976\end{array}$ & $\begin{array}{l}16.1 \\
15.4 \\
21.2 \\
11.4 \\
9.08\end{array}$ & 8.4 & 6.8 & c \\
\hline \multirow[t]{2}{*}{ W2A } & $\begin{array}{l}\text { West Branch } \\
\text { Red River }\end{array}$ & $\begin{array}{l}\text { SE1/4SW1/4 sec. } 19, \text { T. } 30 \text { N., R. } 12 \text { E., } \\
\text { Langlade County, at bridge on } \\
\text { State Highway } 47,3.9 \text { mi west of } \\
\text { Phlox. Wis. }\end{array}$ & 5.20 & $\begin{array}{l}\text { July } 26,1977 \\
\text { Aug. } 16,1977 \\
\text { Oct. 27, } 1977 \\
\text { Sept. 11, } 1978\end{array}$ & $\begin{array}{l}3.15 \\
4.12 \\
3.88 \\
5.58\end{array}$ & 3.8 & 3.2 & c \\
\hline & $\begin{array}{l}\text { West Branch } \\
\text { Red River }\end{array}$ & $\begin{array}{l}\text { SW } 1 / 4 S E 1 / 4 \text { sec. } 30, T .30 \text { N., R. } 12 \text { E., } \\
\text { Langlade County, at bridge on } \\
\text { County Trunk D, } 3.5 \text { mi northwest } \\
\text { of Mattoon, Wis. }\end{array}$ & 6.16 & $\begin{array}{l}\text { July } 26,1977 \\
\text { Aug. } 16,1977 \\
\text { Oct. } 27,1977 \\
\text { Sept. } 11,1978\end{array}$ & $\begin{array}{l}5.12 \\
5.42 \\
7.78 \\
9.01\end{array}$ & 6.0 & 5.1 & c \\
\hline
\end{tabular}

'Based on 1971 report by Wisconsin Department of Natural Resources (DNR). Site lettered "A" was not included in 1971 DNR report.

2 Streambed is dry.

${ }^{3}$ Additional discharge measurements are available.

${ }^{4}$ Negligible discharge--water ponded, unable to measure velocity.

${ }^{5}$ No estimate possible--discharge is primarily effluent. 


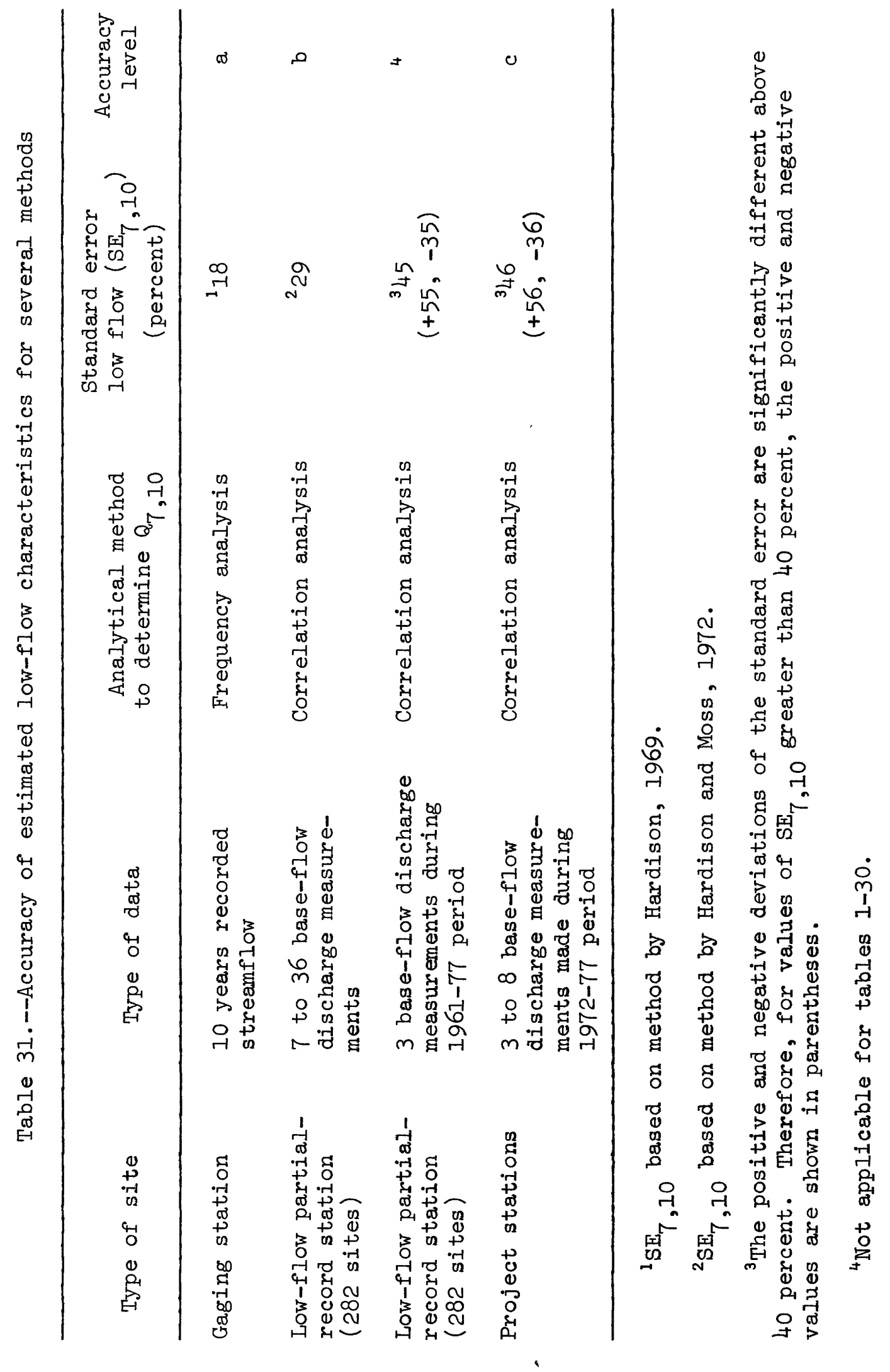


Table 32.--Standard error of estimate for the 10-year low-flow event $\left(\mathrm{SE}_{7,10}\right)$ for Wisconsin basins at low-flow partial-record stations

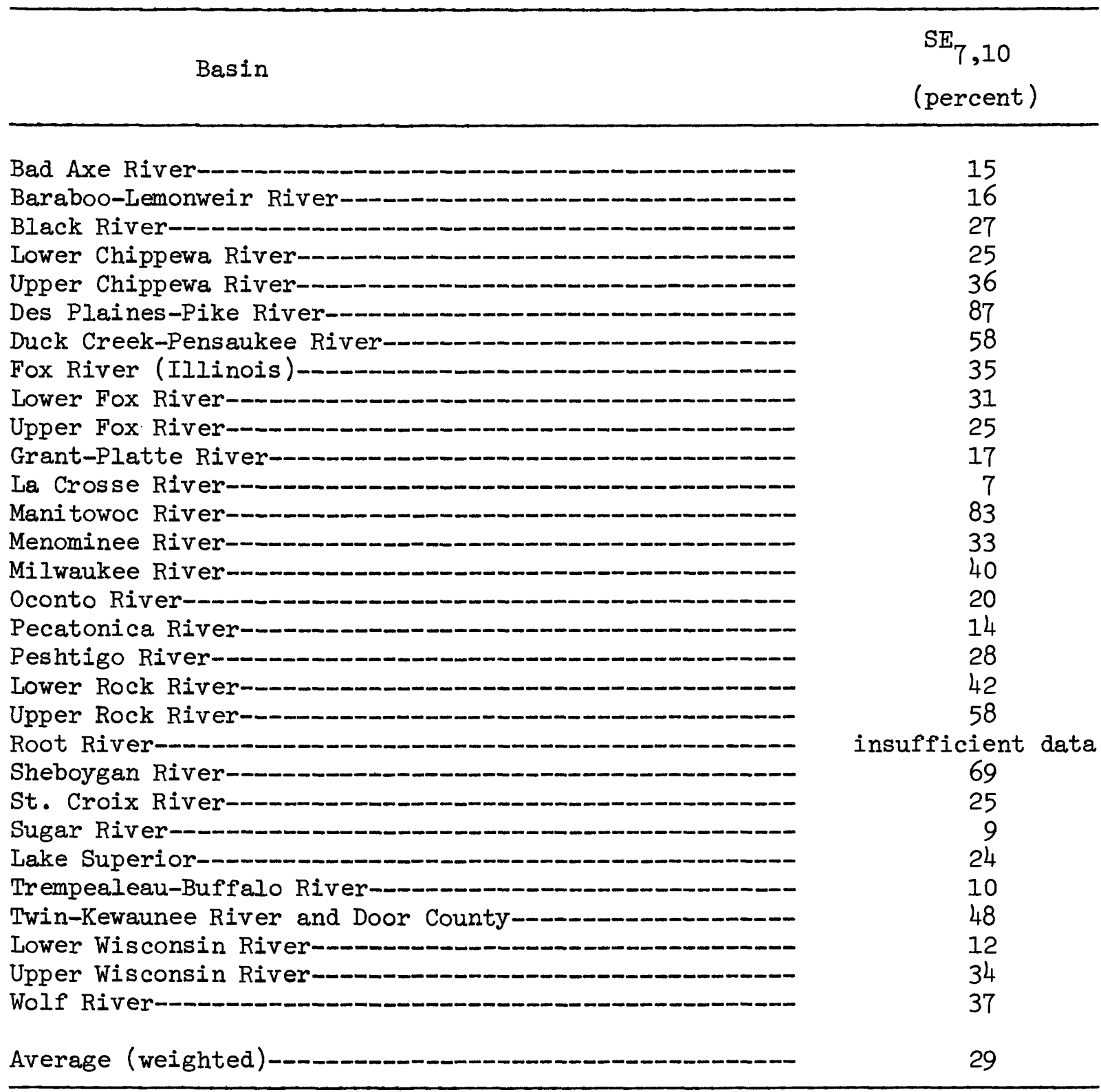




\section{INDEX}

Municipal and Industrial Plants

DNR

station number

Page

Abbotsford-C-

UW77

100

Abrams-C-C-C-C-

DP22

32

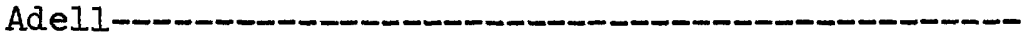

MI18

55

A. F. Schultz Creamery, Phlox-_-

W2A

107

A. G. Cooperative Creamery Co., Arcadia-_-_-_--

BT26

90

A. G. Cooperative Creamery Co. Boiler Plant,

Arcadia-_-_-

BT21

90

Alaskan Motor Inn, Inc., Alaska-_-_-_-_-_-_-

TK29

UR16

A.1 lenton--

Alma Center--

BK35

Almena--

$\mathrm{IC} 74$

Alto Cooperative Creamery, Alto-_-_-_-_-_-_-

URI4

American Motors Company, Kenosha-____-_-_-_-_

DSI6

Amery-_-_-

$\mathrm{SC28}$

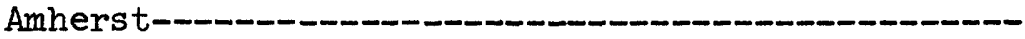

W59

Aniwa--

UW99

Antigo- - - - - - - - - - - - - -

Arcadia-- - -

UW67

Arkansaw Sanitary District No. 1-______-_-_-

BT22

IC93

Arpin

UW116

Ashippun-

UR41

Associated Milk Producers, Inc., Blair-_-_-_-

BTIA

Associated Milk Producers, Inc., Madison-------

LRTA

LFIA

Associated Milk Producers, Inc., Sherwood--

UW55

UW98

Auburndale-_._-__-

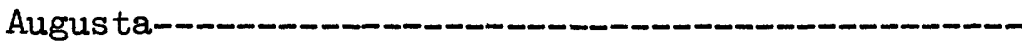

Aunt Nellies Foods, Inc., Clyman-_-_-_-_-_-

Austin Straubel Airport, Green Bay-

Baker Canning Company, Clintonville-_-_-_-

Baker Canning Company, Theresa--

Baker Laboratories, East Troy-_-_-_-_-

Bakerville-_-_____-__-_-

Bain's Country Estates, Sheboygan Falls-_...-.

Baldwin-_-_-_-___

Bals am Lake-_-_______

Bangor-_-_-

Barneveld-_-_-_-_-_-_-

Barron, Jerome Foods, Inc._-_.

Bayfield Fish Hatchery-_-

Bear Creek-_-_-_-

Bear Hole Mine, Shullsburg-_-

Beaver Dam-_-_._-

Belgium-_-__-_-_-_-_-

IC44

UR4 5

LF 48

W44

UR17

F22

UW109

SH18

LC6

$\mathrm{SC} 34$

I9

P3B

IC66

ISIA

W49

GP36

UR63

SH49 


\section{INDEX}

Municipal and Industrial Plants

Belleville-_-_-_-

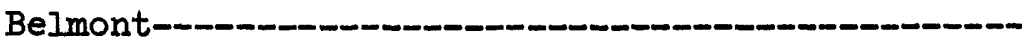

Beloit-_-_-_-__-

Beloit Industrial Park-_____

Beloit Industrial Park-_-

Benton-- -

Berlin-_-_-

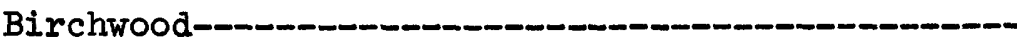

Birnamwood-_-_-

Black Creek-_-_____

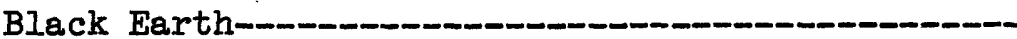

Blair-_-_-_-_-_-

Blanchardville-____C_-

Bloomer-_-_-

Bloomington-

Bohn Sand and Gravel Company, Frederic-_-_-_--

Bonduel-____C_-

Bos cobel-_____-_-

Bowler-_-_-_-_-

Boyceville-_-_-

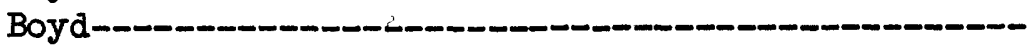

Branch River Country Club-_-

Brandon-_-

Brill Coop Creamery-_-_-

Brill Elementary School-C-

Brillion-_-_-

Bristol-_-

Brodhead

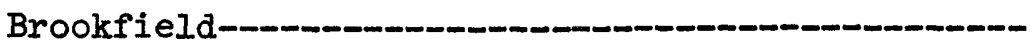

Brooklyn-_-_-_-____-

Brookside Cheese Factory, Abrams-_-

Brownsville-_-

Bruce--C-C-

Brule-- -

Brule Fish Hatchery-

Burlington-C-

Burnett-_-_-_-_-_-_-

Burnett Dairy Coop-_-_

Butler-C-C-C-

Butternut-____________-

$C$ and D Duck Company, Franksville-__

Cadott-_-_-_-_-_-

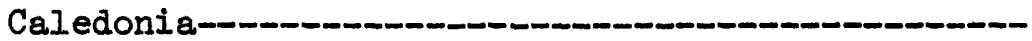

Cambria--C-

Cambridge-_-
DNR

station number

$\underline{\text { Page }}$

S3

P14

84

IRI8

IR2A

LR3A

GP30

UF5

LC52

W39

พ26

LW31

BT18

P26

LC30

GP10

SC16A

W2 4

LW61

W43

LC80

$\mathrm{IC} 43$

M32

UR15

LC60

LC61

M11

DS4

s6

F1

S11

DP23

UR25

UC2

IS3A

IS2A

F8

UR2A

SC17

MI34

UC15

RT22

LC2 4

RT4

LW11

IR34
59

63

66

66

46

41

22

105

104

95

90

59

21

45

76

104

96

105

24

22

51

68

22

22

50

29

84

34

85

33

68

26

88

88

35

71

76

55

27

73

21

72

94

64 


\section{INDEX}

Municipal and Industrial Plants
DNR

station number

LC65

MII

BL9

I4

UR12

S8

DS20

W37

MI1A

TK19

LII

UC21

BLIA

W20

SH 55

SH58

W31

B1I

LC 67

M19

LC12

BKII

SC30

SC37

IR69

W46

UR31

P2

BT13

LF42

UW76

M15

PE9

LWI3

UR 5I

PEIA

B9

IR58

RT11

07

PE7

LW30

UW2A

GP39

LC71
Page

22

54

14

48

67

85

30

105

55

92

49

27

15

104

82

83

105

11

23

51

21

17

77

77

65

105

68

58

90

38

100

51

62

94

70

61

11

65

72

57

61

95

102

46

23 


\section{INDEX}

Municipal and Industrial Plants

Curtiss-_-_-_-_-_-

Dairyland Poultry, Endeavor-_-

Daisy Dairy Company, Poy Sippi-____-__-_-_-_-_

Dale Sanitary District-_-_

Dallas - -

Dane County Hospital________

Darlington-_-

Deerfield-_-_-_-_-_-

Deer Park-_-_-

De Forest-_-

Delafield-_._-

Delavan-_-_-_-__-

Denmark-_-_-____-_-_-

Devil's Head Ski and Recreation Area-_-

Devil's Lake State Park-_-__-

Dickeyville-_-_____-_

Dodgeville-___________-

Dorchester-_-_____

Douglas County Hospital-___-

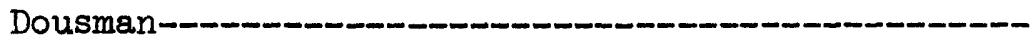

Dresser-_-_-_-

Drummond-_-_-_-_-_-__-

Durand-_-_-_-_-__-__-_-

Eagle Lake Sanitary District-___-

Eagle Picher Mine Company, Blackstone Mine------

Eagle Picher Mine Company, Jewell Mine-_-_-_-_--

Eagle Picher Mine Company, Iinden Mine--_-_-_---

Eagle Picher Mine Company, Schullsburg Mine

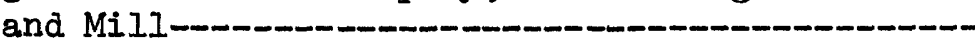

East Troy-_-_-_-_-_-_

Eau Galle Cheese Factory, Eau Galle-_-_._-_-_-

Eden--_-_-

Edgar-_-_-

E. I. Du Pont de Nemours and Company, Barksdale--

Eiler's Cheese Factory, Greenleaf-_-_-_-_-_-_-

Elcho Sanitary District No. 1-_-_-

Eleva-_-

Elkhorn-_-

Ellsworth-_-

Elmwood-_-

Elroy-_-_-_-_-__-_-

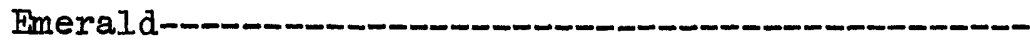

Ettrick-_-_-_-_-_-_-

Evansville-_-_-_-_-_-_-_-

Fairchild
DNR

station number $\quad$ Page

BK15

UF3

W66

W62

LC68

S7

$\mathrm{P} 4$

LR36

$\mathrm{SC} 42$

LR 41

IR2I

IR63

TK6

BL34

BL26 6

GP27

P29

BK10

IS19

LR22

SC24

IS 4 A

LC19

F21

GP2A

P2B

P1B

GP35

F2 4

LC90

UF33

UW58

ISS25

IF $2 A$

WI3

BT6

IR 65

IC 4

LC89

BLI5

$\mathrm{SC} 43$

BK40

S13

LC 41
106

106

23

84

58

64

77

65

63

65

91

15

14

45

59

17

87

64

76

88

21

36

46

60

60

46

36

24

42

100

87

39

104

89

65

20

24

14

77

18

85 


\section{INDEX}

Municipal and Industrial Plants

DNR

station number

Page

Fairwater

UF14A 41

Fall Creek

IC 47

Fall River

UR49

Farmers Creamery Company, Bangor

L8

Fennimore-_-

Fenwood-

GP7

UW $3 A$

B20A

Ferryville--

Fifield Sanitary District-

Florence--_-

Footville-___-___-_-_

Forest-_-

Forestville-_-_

Fort Atkinson-C-

Fox Lake--

Fox River Tractor Company, Appleton--_-

Francis Creek-_-

Franklin-_-_-_-_-

Frank Pure Food Company, Black Creek-_-_-_-_-_-

Frank Pure Food Company, Franksville-_-_-_-_-_-

Frederic-_-

Freedom School District No. 3-_-

Friendship-_-_-_-_-_-

Galesville-_-_-_-_-_-_-_-_-_-_-

Garton Toy Company, Sheboygan-_-_-_-_-_-_-

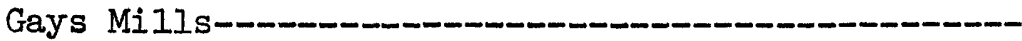

Genoa City Cooperative Milk Association----.----

Germantown--_-_-_-_-

Gibbsville-_-_-

Gilman--------

Gillet-- - - -

Gilson Company, Plymouth-

Gleason-1_-

Glenbeulah-n-n_-

Glenwood City-n-

Glidden Sanitary District-_-

Godfrey Company, Palmyra-

Goodman-- - -

Goodyear Tire and Rubber Company, Sun Prairie----

Grafton-- -

Grande Cheese Company, Oakfield--_-

Grant County Hospital-

Granton-----

Grantsburg--

Grassland Dairy Products, Loyal-- -

Gratiot

$\mathrm{UC16}$

$\mathrm{ME} 6$

LR60

$\mathrm{SC} 4 \mathrm{I}$

TK27

LR2

UR65

LF36

22

70

48

44

102

12

27

53

65

77

93

63

TK10

70

38

RT3

W27

RT25

SC20

92

72

104

DPII

UW108

73

76

BK 41

32

$\mathrm{SH} 12$

LW73

F31

MII 31

$\mathrm{SH} 45$

LC23

06

SH4I

UW 47

SH32

LC79

UCI

LR27

ME7

102

18

80

96

37

55

81

21

57

81

99

81

24

26

IRIA

64

MIII

53

UR5A

66

GP18

55

BK29

71

SC16

45

BK25

18

75

P19

17

59 


\section{INDEX}

Municipal and Industrial Plants

DNR
station number

Greenbush

SH31

80

Green Giant Company, Fox Lake-_-__-

UR64

70

Green Giant Company, Rosendele-_-

UF 43

UF22

Green Valley Cheese Factory, Pulcifer-_-

DP21

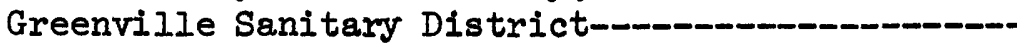

W32

Greenwood-_-

Hammond-

BK21

SC50

Happ's Mobile Home Court, Oshkosh-_-

Hartford-_-_-

Hartland-n--

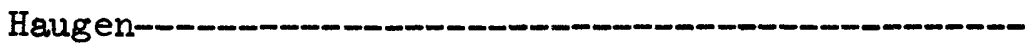

Haven--

Hawkins--

Hawthorn-Mellody Farms Dairy, Whitewater-_--_---

Hayward-_-_-

Hayward Fish Hatchery-

Hazel Green-C-

Highland-C-

Hilbert-C-

Hillsboro-

Hingham--

Hipke Packing Company, Cleveland-

Hixton--

Holland Sanitary District-

Hollandale-C-C

Holmen--

Holy Redeemer College-

Hortonville-C-

Howards Grove, Millersville--

Howard Johnson Restaurant and Motel-

Howard-Suamico School District No. 1--

Hurley

UF 49

UR35

IR20

LC1A

SH11

UC28

IR28

SC8

$\mathrm{SC1B}$

GP3I

LW56

M14

BL27

$\mathrm{SH} 43$

SH9

BT15

IF 45

P31

BK 44

F20

W34

SH13

DS 5

DP6

Hustisford

IS39

UR4

Independence

BT25

International Harvestor Company, Waukesha-------

International Stamping Company, Hartford-------

Iola--

Iowa County Hospital and Home-_-

Iowa Grant High School-_-_-

Iron Belt-_-

Iron Ridge--_-_-

Iron River-C-C

Ixonia-C-C-

Jackson--

Jamestown Sanitary District No. 1-_-

F3A

URIA

W55

P9

P6

IS36

UR32

42

41

32

105

17

78

42

69

63

25

80

28

64

75

78

46

96

51

14

81

80

90

38

60

18

36

105

80

29

32

88

IS 21

UR43

MI24

GP3A 


\section{INDEX}

Municipal and Industrial Plants

Jamestown Sanitary District No. 2

Jamestown Sanitary District No. 3-

Janesville-_-_-_-_-_-_-_-_-_-_-_-

Johnson Wax Company, Sturtevant--

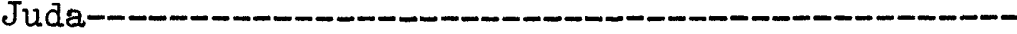

Junction City-_-_-_

Juneau-_-_-_-_-_-_-

Kellnersville--_-_-_-_-_-

Kendall-_-_-

Kewaskum--_-

Kewaunee--_-

Kiel-_-n-_-_-_-_-

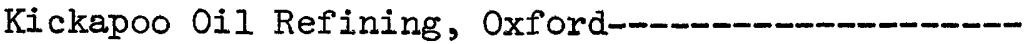

Knapp-_-_-_-_-_-_-_-_-

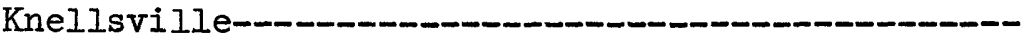

Koppers Company, Superior-_-

Kraft Foods, Beaver Dam-_-_-

Krakow Sanitary District No. 1-

Krier Preserving Company, Belgium-_-_-_-_-_-_-

Krier Preserving Company, Random Lake-_......-...-

Ladish Company, Pleasant Prairie-_-_-_-_-_-_-

Ladysmith-_-_-

La Farge-_-_-_-_-_-

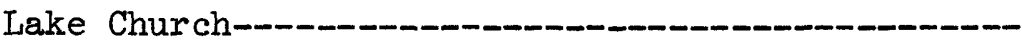

Lake Geneva-_-__-_-_-_

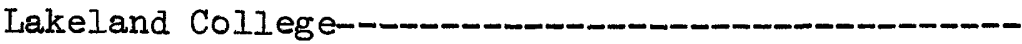

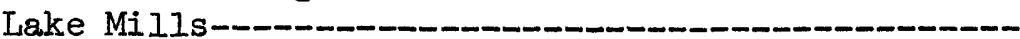

Lake Mills National Fish Hatchery-_-___-

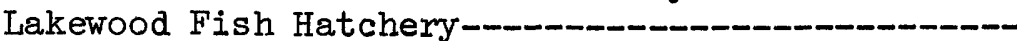

Lamartine-_-_-_-__-

Lancaster-_-_-_-_-_-_-_-_-_-

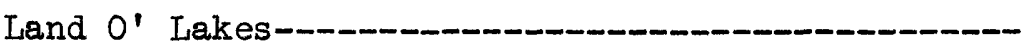

Langlade Fish Hatchery-__-_-

Laona-_-_-_-_-_-_

Lars en--

Larsen Company, Cedar Grove-_-__-

Lebanon-_-_-_-_-_-_-

Le Graves Mobil Home Court-_-

Lena-_-_-

Level Valley Dairy, West Bend-_-_-

Linden--_-_-_-_-_-_-_-

Little Suamico-_-_-_-__-

Livingston-_-_-__-__-

Lodi-_-_-_-_-_-_-

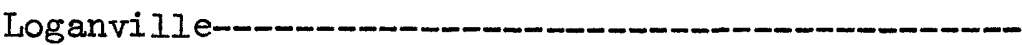

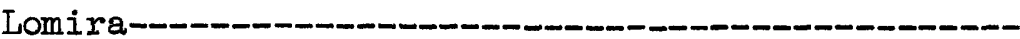

DNR

station number

Page

GP4A

47

GPIA

46

LR12

63

DS13

30

S18

85

UW103

101

UR29

68

TK7

BLI3

MI3

TK17

SH21

UF2B

IC82

SH 57

ISI7

UR62

DP18

$\mathrm{SH} 48$

MI20

DSIA

UC10

IW68

SH 56

F25

SH16

UR71

UR3A

O2A

UF 42

GPII

UW37

OIA

PE5

W74

SH 54

UR 4 A

SH 40

08

MI28

P8A

DP3

GP2I

LW2I

BL30

UR23
92

14

54

92

80

43

24

83

87

70

32

82

55

30

27

96

82

36

80

71

71

57

42

45

99

57

61

107

82

71

81

57

55

59

31

45

95

15

68 


\section{INDEX}

Municipal and Industrial Plants

Loyal--

Luck-- -

Luxemburg-C-

Luxemburg

Lynn Dairy -

Lyons Sanitary District No. 2-_.

Mammoth Spring Canning Company, Oakfield---

Manawa-_-_-_-_-_-_-_-_-_-

Maple Crest Sanitarium, Whitelaw-_-_-

Marathon-_-_-_-_-_-_-

Marengo--

Maribel-

Marion-_-_-_-_-_-_-_-_-_-

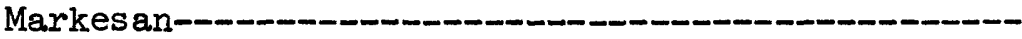

Marshall-_-_-_-_-_-_-_-_-_-_-_-_-_-_-_--

Marshfield-_-_-_-_-_-_-_-_

Mattoon-_-

Mauthe Lake Recreational Area-_-_-_-_-_-_-_--

Mayville-_-_-

Mazomanie-_-_-_-_-_-_-_-_-_-

Medford-_-_-

Meeter Brothers and Company, Union Grove---------

Mellen-_-_-_-_-_-_-_-_-

Melrose--_-_-_-_-_-_-

Melvina-_-_-_-_-_-_-_-

Menomonee Falls-_____C

Menomonie-_-_-_-_-_-_-_-

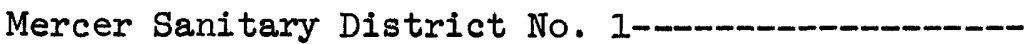

Merril1-_-_-_-_-_-

Merrillan-_-

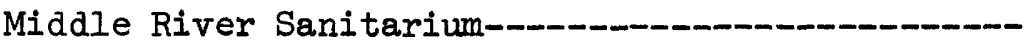

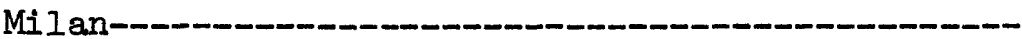

Milk Specialties, Inc., Boscobel-_-_-_-_-_-

Milk Specialties, Inc., Browntown-_-_.--

Milladore-_-_-_-_-_-_-

Milbrew Incorporated, Juneau-_-_-_-

Milltown-_-_-_-_-_-_-_-_-_-_-_-_-_-_-_-

Mineral Point-_-_-

Minocqua-_-_-_-_-

Minong-_-_-_-_-_-_-_-_-

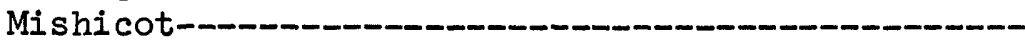

Mondovi-_-_-_-__-__-_-

Monroe--_-_-_-_-_-_-

Montello-_-_-_-_-

Montfort-_-n_-_-_-_-_-

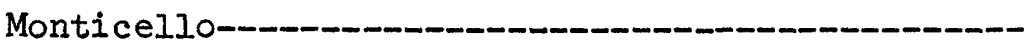

DNR

station number

Page

BK24

17

$\mathrm{SC} 21$

TK18

76

92

TK2A

93

$B K \perp A$

19

F27

UF 41

36

W52

M35

42

UW53

52

IS $5 \mathrm{~A}$

100

TK8

88

W47

UF17

105

UR 56

UW100

W18

MI15

UR21

IW32

BK2

DS2

ISS33

BK38

L12

MI33

IC59

UC12

UW8

BK3 4

IS 20

UW75

LW62

P48

UW102

UR30

SC33

P10

UW 44

SC9

TKI 4

BT8

P56

UF10

IW 54

SI6
41

70

101

104

55

68

95

16

29

87

18

49

55

22

27

99

18

87

100

96

60

101

68

77

59

99

75

92

89

60

41

96

85 


\section{INDEX}

Municipal and Industrial Plants
DNR

station number $\underline{\text { Page }}$

IS 41

IR 40

UW88

SH27

GP8

S10

W77

DS10

F18

ISI6

LW8

F19

URII

UW111

BK5

W16

IR8A

LC28

MI7

F14

S14

M21

BL4

พ8.

SC39

W22

L6

UR57

LW76

BL8

UF 40

F16

UR 44

IR31

UC2 4

IS26

DP13

IW67

SH 51

LR59

S22

IR $4 \mathrm{~A}$

SC27

SC25

$\mathrm{SC} 3 \mathrm{~B}$

BT3
88

65

101

80

44

85

107

30

36

87

94

36

67

102

17

104

66

21

54

35

86

51

13

103

77

104

48

70

96

14

42

35

69

64

28

87

32

96

82

65

85

66

76

76

78

89 


\section{INDEX}

Municipal and Industrial Plants

\begin{tabular}{|c|c|}
\hline $\begin{array}{c}\text { DNR } \\
\text { station number }\end{array}$ & Page \\
\hline SH 46 & 82 \\
\hline BK12 & 17 \\
\hline $\mathrm{S} 1$ & 84 \\
\hline DS7 & 29 \\
\hline IR26 & 64 \\
\hline UF1 & 40 \\
\hline UC8 & 27 \\
\hline GP9 & 45 \\
\hline MEIA & 53 \\
\hline IS42 & 88 \\
\hline PE3 & 61 \\
\hline F15 & 35 \\
\hline BT24 & 90 \\
\hline UW110 & 102 \\
\hline LW27 & 95 \\
\hline GP2 4 & 45 \\
\hline F26 & 36 \\
\hline DS9 & 30 \\
\hline DS6 & 29 \\
\hline IC94 & 25 \\
\hline SH37 & 81 \\
\hline sc26 & 76 \\
\hline UF2 & 40 \\
\hline PE8 & 62 \\
\hline LW18 & 95 \\
\hline W67 & 106 \\
\hline LC72 & 23 \\
\hline UC23 & 28 \\
\hline DP2 & 31 \\
\hline UF 50 & 42 \\
\hline UR20 & 68 \\
\hline $\mathrm{UCl}_{4}$ & 27 \\
\hline UR66 & 71 \\
\hline MI19 & 55 \\
\hline W72 & 107 \\
\hline M26 & 51 \\
\hline UR69 & 71 \\
\hline UW41 & 99 \\
\hline UW51 & 99 \\
\hline SH38 & 81 \\
\hline LW44 & 95 \\
\hline LW46 & 96 \\
\hline IC69 & 23 \\
\hline $\mathrm{P} 4 \mathrm{~B}$ & 60 \\
\hline W75 & 107 \\
\hline
\end{tabular}




\section{INDEX}

Municipal and Industrial Plants

DNR
station number

Rio-n_-

LWI7

Ripon--

UF25

Riverdale Country Club-_._-

River Falls-_-

SH53

Rockdale-_-_-__-_-

Rockland-_-

Rockwood-_-_-

Rogersville Cheese Factory, Rosendale-_-

Rome-----

Rosendale-C-C-n-

Rosholt------

Roxbury-- -

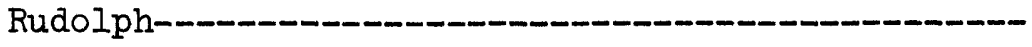

Sacred Heart Seminary, Oneida--

St. Bonaventure Seminary, Sturtevant--

St. Clara Convent, Sinsiniwa-

St. Cloud-- Croix Falls-

St. Croix Falls -

St. Croix Fish Hatchery-

St. Joseph--

St. Mary Springs Academy, Fond du Lac-_-

St. Nazianz--

Salem-----_-

Sauk County Hospital and Home--

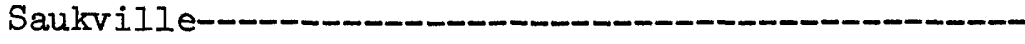

Saxon---- - - -

Scandinavia-C-C-

Schreiber Cheese Company, Green Bay--

Sevastopol Sanitary District No. 1-

Sextonville Sanitary District No. 1-

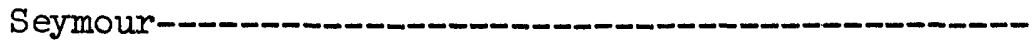

Sharon------- - - - - - - -

Shawano County Hospital-

Sheboygan County Hospital-

Sheboygan Falls---- -

Sheldon---- -

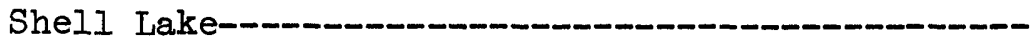

Sherry Dairy--

Sherwood----

Shoto-Two Rivers--C-C-C-

Shullsburg-----

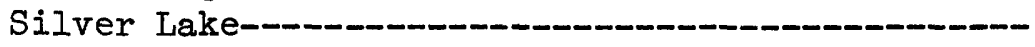

Slinger-c-

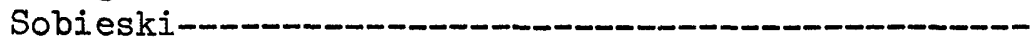

Sobieski Cheese Factory-

Sc49

LR35

L7

TK9

UF 45

IR23

UF 44

W53

LW22

UW105

DP12

DS11

GP29

SH2O

$\mathrm{SC} 3$

SC4B

IIA

UF31

M28

DS8

BL32

MI9

IS37

W56

IF 4A

TK25

LWIA

W25

IR67

W21

SH50

SH2 4

UC20

$\mathrm{SC} 12$

UW4A

MIA

TK5

GP33

F10

UR33

DP 4

DP5 


\section{INDEX}

Municipal and Industrial Plants

DNR
station number

Solon Springs-

SC2

Somerset-.-

SC29

Somers Sanitary District No. 2-_.

DS15

Southern Colony and Training School, Union Grove-

RT18

30

South Hills Country Club-

UF36

Sparta-- -

L5

73

42

UW91

48

Spencer-- -

Spooner-

$\mathrm{SC11}$

Spooner Fish Hatchery-_-_-

$\mathrm{SC} 5 \mathrm{~B}$

101

Spring Valley-n

IC87

Stanley--

Star Prairie-_-

IC40

75

75

SC28A

24

SC3OA

Stella Cheese Company, Clayton-

W29

76

Stetsonville-_-

Stockbridge-_-_-_-_-_-_-_-

Stock's Supper Club, Newton-_-__-

Stokely Van-Camp Inc., Columbus-_-_-_-_-

Stone Lake-------_-

Stoughton-- -

Stratford-------1--

Strum-a-c-n-m-n-

Sturtevant------

Sullivan--n-n-

Summit Cheese Factory, Wilson-

Sun Prairie-_-_-_-

Superior Village-n-C-

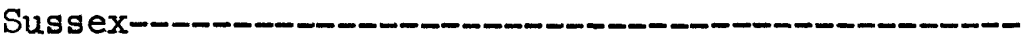

Swift and Company, Pauly Cheese, Green Bay------

Taycheedah---_-_-

Taylor------ - -

Tess Corners--

Tess Corners School-

Theresa-_-

Thorp-

Three Lakes-_-

Thunder River Fish Hatchery-

Tigerton--

Tisch Mills-_-nm-n-

Tomah--

Trent Tube Company, East Troy--

Turtle Lake-- Ta

Twin Lakes--

Union Center--C-C-

Union Grove-

\section{UW72}

UF28

SH8

UR 55

SC2B

IR 43

UW85

BT4

DS12

IR25

LC92

LR32

ISI2

F12

IF $3 A$

UF3IA

BT16

RT9

RT10

UR19

IC38

UW39

PE2A

W40

TK12

BL3

F23

LC7 6

F30

BL16

RT20 


\section{INDEX}

Municipal and Industrial Plants

DNR
station number $\quad$ Page

Uniroyal Incorporation, Stoughton--

IR $5 \mathrm{~A}$

Uni ty--_-

UW89

101

U.S. PIywood Champion, Algoma_-__-

TK3A

93

Valders-_-_-

M30

51

Vernon County Hospital and Home-_-

B14

Verona-_-_-_-_-_-_-_-_-

Vesper-_-_-_-_-_-_-_-

Viroqua-- -

Wabeno--

Waldo-n-m-n-

Wales-C- -

Walworth-C-C-

Waterloo-

Watertown--n-

Weukesha-_-

Waukesha Foundry-_-

Waunakee-n-c-no

Waunakee Canning Corporation-_-__-

Waupun-_-

Wausau-_-__-_-

Wautoma-_-_-_-_-_-_-_-_-_-

Webster-_-_-__-

West Bend-_-_-__-_-

Westboro-_-_-__-

Westby-_-___-___-_-

Western Racine County Sewerage District--_-_-_--

Westfield

Westfield Fish Hatchery-_-__-

West Salem-_-_-

Weyauwega-C-_-_-_-_-

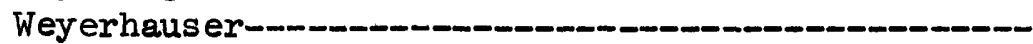

White Clover Dairy Company, Inc., Kaukauna--_---

Whi tehall-_-

Whitelaw-_-_-

Whitewater-_-_-

Wild Rose-_-_._-___-

Wild Rose Fish Hatchery-_-_______-_

Wilson--_-_-_-_-_-

Wilton-_-

Winchester-_-_-_-_-_-

Wind Point-_-_____-

Winter Sanitary District No. 1-

Wisconsin Academy, Columbus-_____

Wisconsin Centrifugal Incorporation, Waukesha----

Wisconsin Dairies Cooperative, Waumandee--------

Wisconsin Home for Women, Taycheedah_-

S9

12

UW114 102

B13 12

05

$\mathrm{SH} 42$

IR2 24

IR70

UR59

UR5

F6

F2A

IR 50

IR 49

UR13

UW13

UF20

SC13

MI6

UC26

B12

F7

UF12

UFIB

IIO

W58

UC6

IF 44

BT19

M34

IR29

W70

W1A

SH 52

LW65

W63

RT30

UC5

UR 48

F1A

BT2A

UF30 


\section{INDEX}

Municipal and Industrial Plants

$\begin{array}{lr}\begin{array}{c}\text { DNR } \\ \text { station number }\end{array} & \text { Page } \\ & \\ \text { UW31 } & 99 \\ \text { W42 } & 105 \\ \text { BL6 } & 13 \\ \text { BL18 } & 14 \\ \text { UC22 } & 28 \\ \text { UW1A } & 102 \\ \text { LC86 } & 24 \\ \text { LF52 } & 39 \\ \text { LW14 } & 94 \\ \text { LW34 } & 95 \\ \text { LR6A } & 66 \\ \text { LW42 } & 95 \\ \text { DP14 } & 32\end{array}$

U.S. GOVERNMENT PRINTING OFFICE: 1979-651-352/ 207 